Geometry $\&$ Topology

Volume 9 (2005) 571-697

Published: 19 April 2005

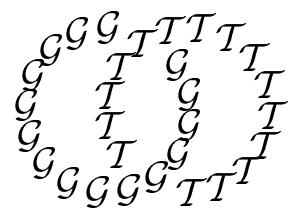

\title{
Counting rational curves of arbitrary shape in projective spaces
}

\author{
AleKsey Zinger \\ Department of Mathematics, Stanford University \\ Stanford, CA 94305-2125, USA \\ Email: azinger@math.stanford.edu \\ URL: http://math.stanford.edu/ azinger/
}

\begin{abstract}
We present an approach to a large class of enumerative problems concerning rational curves in projective spaces. This approach uses analysis to obtain topological information about moduli spaces of stable maps. We demonstrate it by enumerating one-component rational curves with a triple point or a tacnodal point in the three-dimensional projective space and with a cusp in any projective space.
\end{abstract}

\section{AMS Classification numbers Primary: 14N99, 53D99}

Secondary: 55R99

Keywords: Enumerative geometry, projective spaces, rational curves

Proposed: Frances Kirwan

Seconded: Ralph Cohen, Gang Tian
Received: 2 August 2003

Revised: 26 February 2005 


\section{Introduction}

\subsection{Background}

Enumerative geometry of algebraic varieties is a field of mathematics that has been a subject of ongoing research since at least the nineteenth century. Problems in this field are geometric in nature. It has connections to other fields of mathematics as well as to theoretical physics. The general goal of enumerative algebraic geometry is to determine the number of geometric objects that satisfy pre-specified geometric conditions. The objects are often, but not always, complex curves in a smooth algebraic manifold. Such curves would be required to represent a given homology class, to have certain singularities, and to satisfy various contact conditions with respect to a collection of subvarieties. One of the most well-known examples of an enumerative problem is:

Question A If $d$ is a positive integer, what is the number $n_{d}$ of degree$d$ rational curves that pass through $3 d-1$ points in general position in the complex projective plane $\mathbb{P}^{2}$ ?

Since the number of lines through any two distinct points is one, $n_{1}=1$. A little bit of algebraic geometry and topology gives $n_{2}=1$ and $n_{3}=12$. It is far harder to find that $n_{4}=620$, but this number was computed as early as the middle of the nineteenth century; see [16, page 378]. The higher-degree numbers remained unknown until the early 1990s, when a recursive formula for the numbers $n_{d}$ was announced; see [7] and [14].

For more than a hundred years, tools of algebraic geometry had been the dominant force behind progress in enumerative algebraic geometry. However, in [5], Gromov initiated the study of pseudoholomorphic curves in symplectic manifolds and demonstrated their usefulness by obtaining a number of important results in symplectic topology. Since then moduli spaces of stable maps, ie of the parameterizations of pseudoholomorphic curves, have evolved into a powerful tool in enumerative geometry and have become a central object in algebraic geometry. In particular, these moduli spaces lie behind the derivation of the recursive formula for the numbers $n_{d}$ in 7] and [14. The latter work, in fact,

gives a recursive-formula solution to the natural generalization of Question $\mathrm{A}$ to projective spaces of arbitrary dimension:

Question B Suppose $n \geq 2, d$, and $N$ are positive integers, and

$$
\mu \equiv\left(\mu_{1}, \ldots, \mu_{N}\right)
$$


is an $N$-tuple of proper subvarieties of $\mathbb{P}^{n}$ in general position such that

$$
\sum_{l=1}^{N} \operatorname{codim}_{\mathbb{C}} \mu_{l}=d(n+1)+n-3+N .
$$

What is the number $n_{d}(\mu)$ of degree- $d$ rational curves that pass through the subvarieties $\mu_{1}, \ldots, \mu_{N}$ ?

Condition (1.1) is necessary to ensure that the expected answer is finite and not clearly zero. For straightforward geometric reasons (the same ones as for the linearity property and the divisor equation in [14, Section 1]), it is sufficient to solve Question [B] as well as other similar questions, for tuples $\mu$ of linear subspaces of $\mathbb{P}^{n}$ of codimension at least two. Thus, the enumerative formulas given below are stated and proved only for constraints $\mu$ that are points in $\mathbb{P}^{2}$ or points and lines in $\mathbb{P}^{3}$. However, analogous formulas hold for arbitrary constraints $\mu$.

Following [5] and [8], moduli spaces of stable maps into algebraic manifolds became subjects of much research in algebraic geometry. Algebraic geometers usually denote by $\overline{\mathfrak{M}}_{0, N}\left(\mathbb{P}^{n}, d\right)$ the stable-map compactification of the space $\mathfrak{M}_{0, N}\left(\mathbb{P}^{n}, d\right)$ of equivalence classes of degree- $d$ holomorphic maps from $\mathbb{P}^{1}$ with $N$ marked points into $\mathbb{P}^{n}$. These spaces are described as algebraic stacks in [3]. While their cohomology is not entirely understood, it is shown in [11] that the intersections of tautological cohomology classes in $\overline{\mathfrak{M}}_{0, N}\left(\mathbb{P}^{n}, d\right)$ can be computed via explicit recursive formulas. These cohomology classes include all cohomology classes that arise through natural geometric constructions. As an application to enumerative geometry, [1] expresses the number $\left|\mathcal{S}_{1}(\mu)\right|$ of Question $\mathbb{C}$ in terms of intersections of tautological classes in $\overline{\mathfrak{M}}_{0, N}\left(\mathbb{P}^{n}, d\right)$ and then in terms of the numbers $n_{d}$.

Question C If $d$ is a positive integer, what is the number $\left|\mathcal{S}_{1}(\mu)\right|$ of degree-d rational curves that have a cusp and pass through a tuple $\mu$ of $3 d-2$ points in general position in $\mathbb{P}^{2}$ ?

A similar approach to enumerative geometry of plane curves is taken in [15]. Using relationships derived in [2, [15] expresses the "codimension-one" enumerative numbers of rational plane curves, such as those of Questions [C]E in terms of intersection numbers of tautological classes in $\overline{\mathfrak{M}}_{0, N}\left(\mathbb{P}^{n}, d\right)$ and the latter in terms of the numbers $n_{d}$.

Question D If $d$ is a positive integer, what is the number $\frac{1}{6}\left|\mathcal{V}_{1}^{(2)}(\mu)\right|$ of degree- $d$ rational curves that have a triple point and pass through a tuple $\mu$ of $3 d-2$ points in general position in $\mathbb{P}^{2}$ ? 
Question E If $d$ is a positive integer, what is the number $\frac{1}{2}\left|\mathcal{S}_{1}^{(1)}(\mu)\right|$ of degree$d$ rational curves that have a tacnode and pass through a tuple $\mu$ of $3 d-2$ points in general position in $\mathbb{P}^{2}$ ?

Questions $\mathrm{A}$ and $\mathrm{CE}$ can actually be solved using more classical methods of algebraic geometry, as is done in 12 and 13 . However, the derivations in [12] and [13] involve fairly complicated algebraic geometry. In contrast, the computations in [11] and [15] involve much less algebraic geometry and rely on known results, obtained via fairly complicated algebraic geometry elsewhere, including [2], 3], and [8].

The method given in this paper can be used in a straightforward, if somewhat laborious, manner to express the number of rational curves in a complex projective space, that have a $k$-fold point, for example, and pass through a set of constraints in general position, in terms of intersections of tautological classes in the moduli spaces of stable rational maps. In Subsection 1.4, we describe in more detail the scope of the applicability of this method. Its application makes practically no use of algebraic geometry. The method itself relies on a number of technical results, only some of which are contained in this paper, and the rest elsewhere, including [9, 10, 14, 17, 18.

The author would like to thank Tomasz Mrowka and Jason Starr for helpful conversations during the preparation of this manuscript and Izzet Coskun, Joachim Kock, Ravi Vakil, and the referee for comments on early versions of this paper. The author was partially supported by the Clay Mathematics Institute and an NSF Postdoctoral Fellowship. Most of this work was completed at MIT.

\subsection{Outline of the method}

The first step in our approach is to describe a subset $\mathcal{Z}$ of a moduli space of stable rational maps, or of a closely related space, such that the cardinality of $\mathcal{Z}$ is a known multiple of the number we are looking for. We would also like the subset $\mathcal{Z}$ to be the zero set of a reasonably well-behaved section $s$ of a bundle $V$ over a reasonably nice submanifold $\mathcal{S}$ of the ambient space $\overline{\mathcal{M}}$. For example, in the case of Question [C] we might take $\mathcal{S}$ to be the subset of $\mathfrak{M}_{0,1}\left(\mathbb{P}^{2}, d\right)$ consisting of the equivalence classes of maps whose images pass through the $3 d-2$ points in $\mathbb{P}^{2}$ and take $\mathcal{Z}$ to be the subset of $\mathcal{S}$ consisting of the equivalence classes of maps whose differential vanishes at the marked point. Alternatively, we can also allow $\mathcal{Z}$ to be the preimage under a reasonably wellbehaved map $h: \mathcal{S} \longrightarrow \mathcal{X}$ of a submanifold $\Delta$ of $\mathcal{X}$. For example, in the case 
of Question D. we might take $\mathcal{S}$ to be the subset of $\mathfrak{M}_{0,3}\left(\mathbb{P}^{2}, d\right)$ consisting of the equivalence classes of maps $b$ whose images pass through the $3 d-2$ points such that $\mathrm{ev}_{1}(b)=\mathrm{ev}_{2}(b)$, where $\mathrm{ev}_{1}$ and $\mathrm{ev}_{2}$ are the evaluation maps at the first and second marked points of $\mathfrak{M}_{0,3}\left(\mathbb{P}^{2}, d\right)$. We could then take

$$
\mathcal{Z}=\left\{\mathrm{ev}_{1} \times \mathrm{ev}_{3}\right\}^{-1}\left(\Delta_{\mathbb{P}^{2} \times \mathbb{P}^{2}}\right) \cap \mathcal{S}
$$

where $\Delta_{\mathbb{P}^{2} \times \mathbb{P}^{2}}$ denotes the diagonal in $\mathbb{P}^{2} \times \mathbb{P}^{2}$. In the case of Question $\mathbb{E}$, we might take the ambient space to be the projectivization of a natural rank-two bundle over $\overline{\mathfrak{M}}_{0,2}\left(\mathbb{P}^{2}, d\right)$. However, in practice, we will keep track of the points on $\mathbb{P}^{1}$ that get mapped to the constraints, ie there will be marked points labeled by the positive integers $1, \ldots, N$, where $N$ is the number of constraints. The marked points of the domain of a stable map that describe the singularities of the image curve will be labeled by $\widehat{1}, \widehat{2}$, etc.

If $\overline{\mathcal{S}}$ is a smooth compact oriented manifold, $V \longrightarrow \overline{\mathcal{S}}$ is a smooth oriented vector bundle of the same rank as the dimension of $\mathcal{S}$, and $\widetilde{s}: \overline{\mathcal{S}} \longrightarrow V$ is a smooth section, which is transverse to the zero set in $V$, then

$$
{ }^{ \pm}\left|\widetilde{s}^{-1}(0)\right|=\langle e(V), \overline{\mathcal{S}}\rangle,
$$

where ${ }^{ \pm}\left|\widetilde{s}^{-1}(0)\right|$ is the signed cardinality of the set $\widetilde{s}^{-1}(0)$. Equation (1.2) is valid under more general circumstances. In the cases of interest to us, the ambient $\overline{\mathcal{M}}$ is an oriented stratified topological orbifold and $\mathcal{S}$ is a smooth submanifold of the main stratum $\mathcal{M}$ such that $\overline{\mathcal{S}}-\mathcal{S}$ is contained in a finite union of smooth manifolds of dimension less than the dimension of $\mathcal{S}$. Under these assumption, $\mathcal{S}$ determines a homology class in $\overline{\mathcal{M}}$. Furthermore, if $\widetilde{s}$ is a continuous section of $V$ over $\overline{\mathcal{S}}$ and $e(V)$ is the restriction of a cohomology class on $\overline{\mathcal{M}}$, then equality (1.2) still holds. By (1.2), if $s$ is any continuous section of $V$ over $\overline{\mathcal{S}}$ such that $s \mid \mathcal{S}$ is transverse to the zero set and $\mathcal{Z} \equiv s^{-1}(0) \cap \mathcal{S}$ is a finite set, then

$$
{ }^{ \pm}|\mathcal{Z}|=\langle e(V), \overline{\mathcal{S}}\rangle-\mathcal{C}_{\partial \overline{\mathcal{S}}}(s)
$$

where $\mathcal{C}_{\partial \overline{\mathcal{S}}}(s)$ is the $s$-contribution of $\partial \overline{\mathcal{S}}$ to the euler class of $V$. In other words, $\mathcal{C}_{\partial \overline{\mathcal{S}}}(s)$ is the signed number of zeros of a small generic perturbation $\widetilde{s}$ of $s$ that lie near $\partial \overline{\mathcal{S}}$. If the behavior of $s$ near $\partial \overline{\mathcal{S}}$ can be understood, it is reasonable to hope that the number $\mathcal{C}_{\partial \overline{\mathcal{S}}}(s)$ can be computed, at least in terms of evaluations of some cohomology classes. On the other hand, in the case of Question [C] $\overline{\mathcal{S}}$ is a tautological class in the appropriate moduli space of stable maps. Thus, if $e(V)$ is also a tautological class, $\langle e(V), \mathcal{S}\rangle$ is computable, and we are done. Most of the time, however, we will have to describe $\langle e(V), \overline{\mathcal{S}}\rangle$ as the signed cardinality of a subset $\mathcal{Z}^{\prime}$ of a space which is a step closer to a tautological class than $\mathcal{S}$ and apply equation (1.3) with $\mathcal{Z}^{\prime}$. Eventually, we 
will end up with intersections of tautological classes in moduli spaces of stable rational maps.

The topological setup of the previous paragraph is only slightly more general than that of [17. Section 3]. However, it is not sufficient for our purposes. We now present two significant generalizations of this setup. The first is that equation (1.3) makes sense even if the section $s$ is defined only over $\mathcal{S}$ and does not extend over $\overline{\mathcal{S}}-\mathcal{S}$. In such a case, we can use a cutoff function to define a new section $s^{\prime}$ that vanishes on a neighborhood of $\overline{\mathcal{S}}-\mathcal{S}$ and thus extends to a continuous section over $\overline{\mathcal{S}}$. The term $\mathcal{C}_{\partial \overline{\mathcal{S}}}(s)$ is then the signed number of zeros of a small generic perturbation $\widetilde{s}$ of $s^{\prime}$ that lie near $\partial \overline{\mathcal{S}}$. If we can understand the behavior of $s$ near $\partial \overline{\mathcal{S}}$ and choose the cutoff function carefully, it is again reasonable to hope that we can determine the number $\mathcal{C}_{\partial \overline{\mathcal{S}}}(s)$.

The second generalization has a very different flavor. Suppose $\mathcal{S}$ and $\overline{\mathcal{M}}$ are as above and $\mathcal{X}$ is a smooth compact oriented manifold. If $h: \overline{\mathcal{M}} \longrightarrow \mathcal{X}$ is continuous map such that the restriction of $h$ to every stratum of $\overline{\mathcal{M}}$ is smooth, then $h \mid \mathcal{S}$ is a pseudocycle in the sense of [10] and [14, ie it determines an element of $H_{*}(\mathcal{X} ; \mathbb{Z})$. In particular, if $\Delta$ is an immersed compact oriented submanifold of $\mathcal{X}$ such that $\operatorname{dim} \mathcal{S}+\operatorname{dim} \Delta=\operatorname{dim} \mathcal{X}$, there is a well-defined homology-intersection number

$$
\left\langle\left\langle\{h \mid \mathcal{S}\}^{-1}(\Delta)\right\rangle\right\rangle \equiv\left\langle\left\langle h^{-1}(\Delta), \overline{\mathcal{S}}\right\rangle\right\rangle .
$$

If $\mathcal{Y}$ is an immersed compact oriented submanifold of $\mathcal{X}$ such that

$$
[\mathcal{Y}]=[\Delta] \in H_{*}(\mathcal{X} ; \mathbb{Z}), \quad h(\partial \overline{\mathcal{S}}) \cap \mathcal{Y}=\emptyset,
$$

and $h$ is transversal to $\mathcal{Y}$ on $\mathcal{S}$, then

$$
\left\langle\left\langle\{h \mid \mathcal{S}\}^{-1}(\Delta)\right\rangle\right\rangle=\left\langle\left\langle\{h \mid \mathcal{S}\}^{-1}(\mathcal{Y})\right\rangle\right\rangle \equiv{ }^{ \pm}\left|\{h \mid \mathcal{S}\}^{-1}(\mathcal{Y})\right| .
$$

Alternatively, if $\theta$ is a small perturbation of $h$ on a neighborhood of $\overline{\mathcal{S}}$ in $\overline{\mathcal{M}}$,

$$
\left\langle\left\langle\{h \mid \mathcal{S}\}^{-1}(\Delta)\right\rangle\right\rangle={ }^{ \pm}\left|\{\theta \mid \mathcal{S}\}^{-1}(\Delta)\right| .
$$

Thus, if $h: \overline{\mathcal{M}} \longrightarrow \mathcal{X}$ is a continuous map as above such that $h \mid \mathcal{S}$ is transversal to $\Delta$ and $\mathcal{Z} \equiv\{h \mid \mathcal{S}\}^{-1}(\Delta)$ is a finite set,

$$
{ }^{ \pm}|\mathcal{Z}|=\left\langle\left\langle\{h \mid \mathcal{S}\}^{-1}(\Delta)\right\rangle\right\rangle-\mathcal{C}_{\partial \overline{\mathcal{S}}}(h, \Delta),
$$

where $\mathcal{C}_{\partial \overline{\mathcal{S}}}(h, \Delta)$ denotes the $(h, \Delta)$-contribution to the intersection number $\left\langle\left\langle\{h \mid \mathcal{S}\}^{-1}(\Delta)\right\rangle\right.$, ie the signed cardinality of the subset of $\theta^{-1}(\Delta) \cap \mathcal{S}$ consisting of the points that lie near $\partial \overline{\mathcal{S}}$ for a small generic perturbation $\theta$ of $h$ near $\partial \overline{\mathcal{S}}$. If the image of a stratum $\mathcal{Z}_{i}$ of $\partial \overline{\mathcal{S}}$ under $h$ is disjoint from $\Delta$, then clearly $\mathcal{Z}_{i}$ does not contribute to $\mathcal{C}_{\partial \overline{\mathcal{S}}}(h, \Delta)$. If $h$ maps $\mathcal{Z}_{i}$ into $\Delta$, on a neighborhood of $\mathcal{Z}_{i}$ 
we can view $h$ and $\theta$ as vector-bundle sections. Thus, if we can understand the behavior of $h$ near $\overline{\mathcal{S}}$, computing $\mathcal{C}_{\partial \overline{\mathcal{S}}}(h, \Delta)$ is no different than computing $\mathcal{C}_{\partial \overline{\mathcal{S}}}(s)$ in the topological setup presented first. On the other hand, in the cases of interest to us, we will be able to find a submanifold $\mathcal{Y}$ as in (1.4) such that ${ }^{ \pm}\left|\{h \mid \mathcal{S}\}^{-1}(\mathcal{Y})\right|$ can be expressed as evaluation of tautological classes on $\overline{\mathcal{S}}$; see Subsection 4.1 for example. If $\overline{\mathcal{S}}$ itself is not a tautological class in a moduli space of rational stable maps, we will have to describe ${ }^{ \pm}\left|\{h \mid \mathcal{S}\}^{-1}(\mathcal{Y})\right|$ as the signed cardinality of a subset $\mathcal{Z}^{\prime}$ of a space which is a step closer to a tautological class than $\mathcal{S}$ and apply equation (1.3) or (1.5) with $\mathcal{Z}^{\prime}$. Eventually, we will end up with intersections of tautological classes on moduli spaces of stable rational maps.

In Subsection 2.2, we describe our topological assumptions on $\mathcal{S}, \overline{\mathcal{M}}$, and the behavior of $s$ or $h$ near $\partial \overline{\mathcal{S}}$. These assumptions imply that the sets $s^{-1}(0) \cap \mathcal{S}$ and $\{h \mid \mathcal{S}\}^{-1}(\Delta)$ are finite. Roughly speaking, we require that $\partial \overline{\mathcal{S}}$ be contained in a finite union of smooth manifolds $\mathcal{Z}_{i}$ such that near each $\mathcal{Z}_{i}$ the section $s$ or the map $h$ can be approximated by a polynomial map between vector bundles over $\mathcal{Z}_{i}$. The polynomial map may contain terms of negative degree. Propositions $2.18 \mathrm{~A}$ and $2.18 \mathrm{~B}$ of Subsection 2.3 give an inductive procedure for computing the contribution from each space $\mathcal{Z}_{i}$ to $\mathcal{C}_{\partial \overline{\mathcal{S}}}(s)$ or to $\mathcal{C}_{\partial \overline{\mathcal{S}}}(h, \Delta)$ in good cases. The two propositions describe how to set up a finite tree with topological intersection numbers assigned to the nodes and with integer weights assigned to the edges. The root of the tree is assigned the first term on the right-hand side of (1.3) or (1.5). The number on the left-hand side of (1.3) or (1.5) is a weighted sum of the numbers at the nodes. The weight of the number assigned to a node is the product of the weights assigned to the edges between the node and the root.

Remark The method presented in Subsection 2.2 is an improvement over that of Section 3 in [17. even for the basic topological setup of the second paragraph of this subsection. In particular, its use does not require applications of the Implicit Function Theorem (IFT) to describe a neighborhood of $\partial \overline{\mathcal{S}}$ in $\overline{\mathcal{S}}$. The complexity of applying the IFT increases rapidly with the dimension of the boundary strata, as a comparison between [17, Subsection 5.4] and 19. Subsection 2.3] suggests.

In order to apply the topological method of this paper to enumerative problems, we use Lemma 3.4 and Proposition 3.5. The former is a rather elementary result in complex geometry and implies that various bundle sections over smooth strata of moduli spaces of stable rational maps are transverse to the zero set. 
The latter depends on the explicit construction of the gluing map in [18] and describes the behavior of these bundle sections near the boundary of each stratum. In many cases, Proposition 3.5. combined with Lemma 3.4, implies that natural submanifolds $\mathcal{S}$ of moduli spaces of stable rational maps, or of closely related spaces, that are needed for counting singular rational curves are well-behaved near $\partial \overline{\mathcal{S}}$ and that the behavior near $\partial \overline{\mathcal{S}}$ of various natural vector-bundle sections over $\mathcal{S}$ can be approximated by polynomials.

\subsection{Computed examples}

We now describe the main enumerative results derived in this paper using the computational method outlined above. These are the enumerations of triplepointed and of tacnodal rational one-component curves in $\mathbb{P}^{3}$ and of rational one-component cuspidal curves in $\mathbb{P}^{n}$ that pass through a collection of constraints in general position. The reason we choose these examples is that they illustrate all aspects of our method and lead to new results. The numerical values of some low-degree numbers can be found at the end of the paper. Note that our low-degree numbers pass the standard classical checks; see Section 8

We start by giving a formula describing the number of cuspidal curves in $\mathbb{P}^{n}$. This is actually the least interesting example of the three mentioned, as it should have really been done in 20. However, the solution to this example is easier to state and explain than the answers to the two other primary examples.

Theorem 1.1 Suppose $n \geq 2, d \geq 1, N \geq 0$, and $\mu=\left(\mu_{1}, \ldots, \mu_{N}\right)$ is an $N$-tuple of proper subvarieties of $\mathbb{P}^{n}$ in general position such that

$$
\sum_{l=1}^{N} \operatorname{codim}_{\mathbb{C}} \mu_{l}=d(n+1)-2+N .
$$

The number of rational cuspidal degree- $d$ curves that pass through the constraints $\mu$ is given by

$$
\left|\mathcal{S}_{1}(\mu)\right|=\sum_{k=1}^{2 k \leq n+2}(-1)^{k-1}(k-1) ! \sum_{l=0}^{n+2-2 k}\left(\begin{array}{c}
n+1 \\
l
\end{array}\right)\left\langle a_{\widehat{0}}^{l} \eta_{\widehat{0}, n+2-2 k-l}, \overline{\mathcal{V}}_{k}(\mu)\right\rangle .
$$

We now explain the notation involved in the statement of Theorem 1.1. The compact oriented topological manifold $\overline{\mathcal{V}}_{k}(\mu)$, which in general may be an orbifold, consists of unordered $k$-tuples of stable rational maps of total degree $d$. Each map comes with a special marked point $(i, \infty)$. All these marked points 
are mapped to the same point in $\mathbb{P}^{n}$. In particular, there is a well-defined evaluation map

$$
\mathrm{ev}_{\widehat{0}}: \overline{\mathcal{V}}_{k}(\mu) \longrightarrow \mathbb{P}^{n}
$$

which sends each tuple of stable maps to the value at (any) one of the special marked points. We also require that the union of the images of the maps in each tuple intersect each of the constraints $\mu_{1}, \ldots, \mu_{N}$. In fact, the elements in the tuple carry a total of $N$ marked points, $y_{1}, \ldots, y_{N}$, in addition to the $k$ special marked points. These marked points are mapped to the constraints $\mu_{1}, \ldots, \mu_{N}$, respectively. Roughly speaking, each element of $\overline{\mathcal{V}}_{k}(\mu)$ corresponds to a degree- $d$ rational curve in $\mathbb{P}^{n}$, which has at least $k$ irreducible components, and $k$ of the components meet at the same point in $\mathbb{P}^{n}$. The precise definition of the spaces $\overline{\mathcal{V}}_{k}(\mu)$ can be found in Subsection 3.1.

The cohomology classes $a_{\widehat{0}}$ and $\eta_{\widehat{0}, l}$ are tautological classes in $\overline{\mathcal{V}}_{k}(\mu)$. In fact,

$$
a_{\widehat{0}}=\operatorname{ev}_{\widehat{0}}^{*} c_{1}\left(\mathcal{O}_{\mathbb{P}^{n}}(1)\right) .
$$

Let $\overline{\mathcal{V}}_{k}^{\prime}(\mu)$ be the oriented topological orbifold defined as $\overline{\mathcal{V}}_{k}(\mu)$, except without specifying the marked points $y_{1}, \ldots, y_{N}$ mapped to the constraints $\mu_{1}, \ldots, \mu_{N}$. Then, there is well-defined forgetful map,

$$
\pi_{k}: \overline{\mathcal{V}}_{k}(\mu) \longrightarrow \overline{\mathcal{V}}_{k}^{\prime}(\mu)
$$

which drops the marked points $y_{1}, \ldots, y_{N}$ and contracts the unstable components. Let

$$
\eta_{\widehat{0}, l}^{\prime} \in H^{2 l}\left(\overline{\mathcal{V}}_{k}^{\prime}(\mu)\right)
$$

be the sum of all degree- $l$ monomials in

$$
\psi_{(1, \infty)}, \ldots, \psi_{(k, \infty)},
$$

where $\psi_{(i, \infty)}$ is the first chern class of the universal cotangent line bundle for the marked point $(i, \infty) \in \mathbb{P}^{1}$. Since $\overline{\mathcal{V}}_{k}^{\prime}(\mu)$ is a collection of unordered $k$-tuples, a priori $\psi_{(i, \infty)}$ may not be well defined as an element of $\overline{\mathcal{V}}_{k}^{\prime}(\mu)$. However, it is easy to see that every symmetric polynomial in $\psi_{(1, \infty)}, \ldots, \psi_{(k, \infty)}$ is well defined. We put

$$
\eta_{\widehat{0}, l}=\pi_{k}^{*} \eta_{\widehat{0}, l}^{\prime} \in H^{2 l}\left(\overline{\mathcal{V}}_{k}(\mu)\right) .
$$

In Subsection 3.1] we give a definition of $\eta_{l}$ that does not involve the projection map $\pi_{k}$. The algorithm of [1] for computing intersections of tautological classes in $\overline{\mathfrak{M}}_{0, N}\left(d, \mathbb{P}^{n}\right)$ applies, with no change, to computing the intersection numbers involved in the statement of Theorem 1.1

We will call top intersections of tautological classes on $\overline{\mathfrak{M}}_{0, k}\left(\mathbb{P}^{n}, d\right)$ and on closely related spaces, such as $\overline{\mathcal{V}}_{k}(\mu)$ and projectivizations of natural vector 
bundles over $\overline{\mathcal{V}}_{k}(\mu)$, level 0 numbers. All such numbers can be computed using the algorithm of [11. Counts of rational curves with $s$ basic singularity conditions will be called level $s$ numbers. Every level $s$ number, with $s>0$, can be written in the form (1.3) or (1.5) such that the middle term is a level $(s-1)$ number. For example, the number $\left|\mathcal{S}_{1}(\mu)\right|$ of Theorem 1.1] is a level 1 number.

Counts of rational curves with a triple point or a tacnode are level 2 numbers. Indeed, the sets of such curves are subsets of the space of one-component rational curves with a node. Counts of such curves are level 1 numbers, since the next level down are the rational curves that pass through the given constraints; see the first paragraph of Subsection 1.2 Thus, the first two theorems below express level 2 numbers in terms of level 1 numbers. After stating them, we give some clarification on the notation involved and then state several lemmas that express the relevant level 1 numbers in terms of level 0 numbers.

Theorem 1.2 Let $d, p$, and $q$ be nonnegative integers such that $2 p+q=$ $4 d-3$. The number of rational one-component degree- $d$ curves that have a triple point and pass through a tuple $\mu$ of $p$ points and $q$ lines in general position in $\mathbb{P}^{3}$ is $\frac{1}{6}\left|\mathcal{V}_{1}^{(2)}(\mu)\right|$, where

$$
\begin{aligned}
\left|\mathcal{V}_{1}^{(2)}(\mu)\right|=\mid & \mathcal{V}_{1}^{(1)}\left(\mu+H^{0}\right) \mid+\left\langle a_{\widehat{0}}, \overline{\mathcal{V}}_{1}^{(1)}\left(\mu+H^{1}\right)\right\rangle+\left\langle 16 a_{\widehat{0}}+8 \eta_{\widehat{0}, 1}, \overline{\mathcal{S}}_{1}(\mu)\right\rangle \\
& +2\left|\mathcal{V}_{2}^{(1)}(\mu)\right|-\left\langle(12-d) a_{\widehat{0}}^{2}+8 a_{\widehat{0}} \eta_{\widehat{0}, 1}+2 \eta_{\widehat{0}, 1}^{2}, \overline{\mathcal{V}}_{1}^{(1)}(\mu)\right\rangle-2\left|\mathcal{S}_{2}(\mu)\right|
\end{aligned}
$$

Theorem 1.3 Let $d, p$, and $q$ be nonnegative integers such that $2 p+q=$ $4 d-3$. The number of rational one-component degree- $d$ curves that have a tacnodal point and pass through a tuple $\mu$ of $p$ points and $q$ lines in general position in $\mathbb{P}^{3}$ is $\frac{1}{2}\left|\mathcal{S}_{1}^{(1)}(\mu)\right|$, where

$$
\begin{aligned}
\left|\mathcal{S}_{1}^{(1)}(\mu)\right|=\left\langle 6 a_{\widehat{0}}^{2}+\eta_{\widehat{0}, 1}^{2}, \overline{\mathcal{V}}_{1}^{(1)}(\mu)\right\rangle+ & \left\langle 4 a_{\widehat{0}}+\frac{1}{2} \eta_{\widehat{0}, 1}, \overline{\mathcal{V}}_{2}^{(1,1)}(\mu)\right\rangle+7\left|\mathcal{S}_{2}(\mu)\right| \\
& -\left\langle 20 a_{\widehat{0}}+19 \eta_{\widehat{0}, 1}, \overline{\mathcal{S}}_{1}(\mu)\right\rangle-2\left|\mathcal{V}_{2}^{(1)}(\mu)\right| .
\end{aligned}
$$

We define the spaces $\overline{\mathcal{V}}_{k}^{(1)}(\mu)$ as follows. Let $\mathcal{V}_{k}^{(1)}(\mu)$ be the space of $k$-tuples of stable maps as in the construction of the space $\overline{\mathcal{V}}_{k}(\mu)$, but with the following exceptions. Every element of each $k$-tuple lies in the main stratum of the appropriate moduli space of stable maps, ie the domain of the map is $\mathbb{P}^{1}$. Furthermore, one of the elements of each $k$-tuple $b$ carries a special marked point, labeled by $\hat{1}$, and the value of the map at this point is $\operatorname{ev}_{\widehat{0}}(b)$. In the space $\mathcal{V}_{2}^{(1,1)}(\mu)$ each of the two components carries a special marked point, one of which is labeled by $\widehat{1}$ and the other by $\widehat{2}$. Furthermore, $\operatorname{ev}_{\widehat{1}}(b)=\operatorname{ev}_{\widehat{2}}(b)$ for 
all 2-tuples $b$ in $\mathcal{V}_{2}^{(1,1)}(\mu)$. The spaces $\overline{\mathcal{V}}_{k}^{(1)}(\mu)$ and $\overline{\mathcal{V}}_{2}^{(1,1)}(\mu)$ are the closures of the spaces $\mathcal{V}_{k}^{(1)}(\mu)$ and $\mathcal{V}_{2}^{(1,1)}(\mu)$ in the unions of the appropriate products of moduli spaces of stable rational maps. We denote by $\mathcal{S}_{*}^{*}(\mu)$ the subspace of $\mathcal{V}_{*}^{*}(\mu)$ consisting of tuples of maps with the simplest possible additional natural singularity. For example, the differential of every element of $\mathcal{S}_{1}(\mu)$ vanishes at $(1, \infty)$. The set $\mathcal{S}_{2}(\mu)$ is described in detail by Lemma 1.5] Figure 11 depicts the images of typical elements of these spaces as well as of $\mathcal{V}_{2,(0,1)}^{(1 ; 0,1)}(\mu)$, which appears in a relationship between level 1 numbers; see the remark following the proof of Lemma 5.8. We give formal definitions of all these spaces in Subsections 4.1, 4.4, and 5.1. Finally, $\mu+H^{r}$ denotes the $(N+1)$-tuple of constrains $\left(\mu_{1}, \ldots, \mu_{N}, H^{r}\right)$, where $H^{r}$ is a generic linear subspace of $\mathbb{P}^{n}$ of complex dimension $r$.

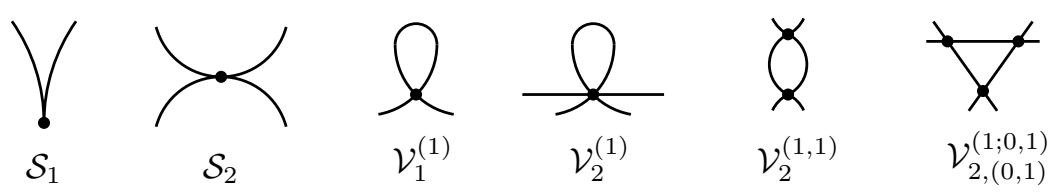

Figure 1: Images of typical elements of $\mathcal{S}_{*}$ and $\mathcal{V}_{*}^{*}$

Lemma 1.4 Suppose $d, p$, and $q$ are nonnegative integers such that $2 p+q=$ $4 d-3$ and $\mu$ is a tuple of $p$ points and $q$ lines in general position in $\mathbb{P}^{3}$. The number of rational connected two-component degree- $d$ curves that pass through the constraints $\mu$ and such that one of the components of each curve has a node and the other component is attached at the node of the first component as depicted in Figure [1 is $\frac{1}{2}\left|\mathcal{V}_{2}^{(1)}(\mu)\right|$, where

$$
\begin{aligned}
\left|\mathcal{V}_{2}^{(1)}(\mu)\right|=\left|\mathcal{V}_{2}\left(\mu+H^{0}\right)\right|+\left\langle a_{\widehat{0}}, \overline{\mathcal{V}}_{2}\left(\mu+H^{1}\right)\right\rangle+3\left|\mathcal{V}_{3}(\mu)\right| \\
-\left\langle(12-d) a_{\widehat{0}}^{2}+4 a_{\widehat{0}} \eta_{\widehat{0}, 1}+2 \eta_{\widehat{0}, 2}-\eta_{\widehat{0}, 1}^{2}, \overline{\mathcal{V}}_{2}(\mu)\right\rangle .
\end{aligned}
$$

Lemma 1.5 Suppose $d, p$, and $q$ are nonnegative integers such that $2 p+q=$ $4 d-3$ and $\mu$ is a tuple of $p$ points and $q$ lines in general position in $\mathbb{P}^{3}$. The number of rational connected two-component degree- $d$ curves that pass through the constraints $\mu$ and have a tacnodal point is given by

$$
\left|\mathcal{S}_{2}(\mu)\right|=\left\langle 6 a_{\widehat{0}}^{2}+4 a_{\widehat{0}} \eta_{\widehat{0}, 1}+\eta_{\widehat{0}, 2}, \overline{\mathcal{V}}_{2}(\mu)\right\rangle-3\left|\mathcal{V}_{3}(\mu)\right| .
$$

Lemma 1.6 Suppose $d, p$, and $q$ are nonnegative integer such that $2 p+q=$ $4 d-3$ and $\mu$ is a tuple of $p$ points and $q$ lines in general position in $\mathbb{P}^{3}$. 
(1) The number of rational connected two-component degree-d curves, with the components arranged in a circle as in Figure 1 that pass through the constraints $\mu$ and have one of the nodes on a generic hyperplane is given by

$$
\frac{1}{2}\left\langle a_{\widehat{0}}, \overline{\mathcal{V}}_{2}^{(1,1)}(\mu)\right\rangle=\left\langle a_{\widehat{0}}, \overline{\mathcal{V}}_{2}\left(\mu+\left\{H^{1}: H^{2}\right\}\right)\right\rangle-\left\langle 4 a_{\widehat{0}}^{2}+a_{\widehat{0}} \eta_{\widehat{0}, 1}, \overline{\mathcal{V}}_{2}(\mu)\right\rangle .
$$

(2) Furthermore,

$$
\begin{aligned}
\frac{1}{2}\left\langle\eta_{\widehat{0}, 1}, \overline{\mathcal{V}}_{2}^{(1,1)}(\mu)\right\rangle=\left\langle\eta_{\widehat{0}, 1},\right. & \left.\overline{\mathcal{V}}_{2}\left(\mu+\left\{H^{1}: H^{2}\right\}\right)\right\rangle+\left|\mathcal{V}_{2}\left(\mu+H^{0}\right)\right| \\
& +\left\langle a_{\widehat{0}}, \overline{\mathcal{V}}_{2}\left(\mu+H^{1}\right)\right\rangle+d\left\langle a_{\widehat{0}}^{2}, \overline{\mathcal{V}}_{2}(\mu)\right\rangle-3\left|\mathcal{V}_{3}(\mu)\right|
\end{aligned}
$$

Lemma 1.7 Suppose $d, p$, and $q$ are nonnegative integers such that $2 p+q=$ $4 d-3$ and $\mu$ is a tuple of $p$ points and $q$ lines in general position in $\mathbb{P}^{3}$.

(1) The number of rational degree- $d$ curves that pass through the constraints $\mu$ and have a cusp on a generic hyperplane is given by

$$
\left\langle a_{\widehat{0}}, \overline{\mathcal{S}}_{1}(\mu)\right\rangle=\left\langle 6 a_{\widehat{0}}^{3} \eta_{\widehat{0}, 1}+4 a_{\widehat{0}}^{2} \eta_{\widehat{0}, 1}^{2}+a_{\widehat{0}} \eta_{\widehat{0}, 1}^{3}, \overline{\mathcal{V}}_{1}(\mu)\right\rangle-\left\langle 4 a_{\widehat{0}}^{2}+a_{\widehat{0}} \eta_{\widehat{0}, 1}, \overline{\mathcal{V}}_{2}(\mu)\right\rangle .
$$

(2) Furthermore,

$$
\left\langle\eta_{\widehat{0}, 1}, \overline{\mathcal{S}}_{1}(\mu)\right\rangle=\left\langle 4 a_{\widehat{0}}^{3} \eta_{\widehat{0}, 1}+6 a_{\widehat{0}}^{2} \eta_{\widehat{0}, 1}^{2}+4 a_{\widehat{0}} \eta_{\widehat{0}, 1}^{3}+\eta_{\widehat{0}, 1}^{4}, \overline{\mathcal{V}}_{1}(\mu)\right\rangle-\left|\mathcal{V}_{3}(\mu)\right|
$$

Lemma 1.8 Suppose $d, p$, and $q$ are nonnegative integers and $\mu$ is a tuple of $p$ points and $q$ lines in general position in $\mathbb{P}^{3}$.

(1) If $2 p+q=4 d-1$, the number of rational one-component degree- $d$ curves that pass through the constraints $\mu$ and have a node is $\frac{1}{2}\left|\mathcal{V}_{1}^{(1)}(\mu)\right|$, where

$$
\left|\mathcal{V}_{1}^{(1)}(\mu)\right|=\left\langle(2 d-6) a_{\widehat{0}}^{2}-4 a_{\widehat{0}} \eta_{\widehat{0}, 1}-\eta_{\widehat{0}, 1}^{2}, \overline{\mathcal{V}}_{1}(\mu)\right\rangle+\left|\mathcal{V}_{2}(\mu)\right| .
$$

(2) If $2 p+q=4 d-2$, the number of rational one-component degree- $d$ curves that pass through the constraints $\mu$ and have a node on a generic hyperplane is $\frac{1}{2}\left\langle a_{\widehat{0}}, \overline{\mathcal{V}}_{1}^{(1)}(\mu)\right\rangle$, where

$$
\begin{aligned}
\left\langle a_{\widehat{0}}, \overline{\mathcal{V}}_{1}^{(1)}(\mu)\right\rangle=\left\langle(2 d-6) a_{\widehat{0}}^{3}-4 a_{\widehat{0}}^{2} \eta_{\widehat{0}, 1}-a_{\widehat{0}} \eta_{\widehat{0}, 1}^{2}, \overline{\mathcal{V}}_{1}(\mu)\right\rangle & \\
& +\left\langle a_{\widehat{0}}^{2}, \overline{\mathcal{V}}_{1}\left(\mu+H^{1}\right)\right\rangle+\left\langle a_{\widehat{0}}, \overline{\mathcal{V}}_{2}(\mu)\right\rangle .
\end{aligned}
$$

(3a) If $2 p+q=4 d-3$, the number of rational one-component degree- $d$ curves that pass through the constraints $\mu$ and have a node on a generic line is $\frac{1}{2}\left\langle a_{\widehat{0}}^{2}, \overline{\mathcal{V}}_{1}^{(1)}(\mu)\right\rangle$, where

$$
\left\langle a_{\widehat{0}}^{2}, \overline{\mathcal{V}}_{1}^{(1)}(\mu)\right\rangle=2\left\langle a_{\widehat{0}}^{3}, \overline{\mathcal{V}}_{1}\left(\mu+H^{1}\right)\right\rangle-\left\langle 4 a_{\widehat{0}}^{3} \eta_{\widehat{0}, 1}+a_{\widehat{0}}^{2} \eta_{\widehat{0}, 1}^{2}, \overline{\mathcal{V}}_{1}(\mu)\right\rangle+\left\langle a_{\widehat{0}}^{2}, \overline{\mathcal{V}}_{2}(\mu)\right\rangle
$$


(3b) Furthermore,

$$
\begin{aligned}
&\left\langle a_{\widehat{0}} \eta_{\widehat{0}, 1}, \overline{\mathcal{V}}_{1}^{(1)}(\mu)\right\rangle=\left\langle a_{\widehat{0}} \eta_{\widehat{0}, 1},\right.\left.\overline{\mathcal{V}}_{1}\left(\mu+H^{0}\right)\right\rangle+\left\langle a_{\widehat{0}}^{2} \eta_{\widehat{0}, 1}, \overline{\mathcal{V}}_{1}\left(\mu+H^{1}\right)\right\rangle \\
&+d\left\langle a_{\widehat{0}}^{3} \eta_{\widehat{0}, 1}, \overline{\mathcal{V}}_{1}(\mu)\right\rangle-\left\langle 4 a_{\widehat{0}}^{2}+a_{\widehat{0}} \eta_{\widehat{0}, 1}, \overline{\mathcal{V}}_{2}(\mu)\right\rangle \\
&\left\langle\eta_{\widehat{0}, 1}^{2}, \overline{\mathcal{V}}_{1}^{(1)}(\mu)\right\rangle=\left\langle\eta_{\widehat{0}, 1}^{2}, \overline{\mathcal{V}}_{1}\left(\mu+H^{0}\right)\right\rangle+\left\langle a_{\widehat{0}} \eta_{\widehat{0}, 1}^{2}, \overline{\mathcal{V}}_{1}\left(\mu+H^{1}\right)\right\rangle \\
&+\left\langle 4 a_{\widehat{0}}^{3} \eta_{\widehat{0}, 1}+d \cdot a_{\widehat{0}}^{2} \eta_{\widehat{0}, 1}^{2}, \overline{\mathcal{V}}_{1}(\mu)\right\rangle-\left|\mathcal{V}_{3}(\mu)\right| .
\end{aligned}
$$

Every term on the right-hand side of each expression in Lemmas 1.4 1.8 is a level 0 number, ie it is a top intersection of tautological classes in a product of moduli spaces of stable maps and thus is computable via the explicit formulas of [11. We define the space $\mathcal{V}_{2}\left(\mu+\left\{H^{1}: H^{2}\right\}\right)$ in the same way as $\mathcal{V}_{2}\left(\mu+H^{1}+H^{2}\right)$, with the only exception that we require $H_{1}$ and $H_{2}$ to lie on different elements of the tuple $b$.

Remark If one were to derive a completely general recursive formula for counting rational curves with singularities, no separate formula would be necessary for generalizations of Lemma 1.6 part (2), Lemma 1.7 part (2), and Lemma 1.8 part (3b). Using [11], one can express all classes $\eta_{*, *}^{*}$ on products of moduli spaces of stable rational maps in terms of subspaces of possibly other products of moduli spaces of stable rational maps that consist of stable maps sending their marked points to various constraints in $\mathbb{P}^{n}$. In many cases, using Proposition 3.5 one can thus express evaluations of the classes $\eta_{*, *}^{*}$ on a space $\overline{\mathcal{S}}$ of maps that represent curves with certain singularities in terms of the numbers of singular curves that pass through various constraints. Furthermore, the level of the latter numbers will be no higher than that of $\mathcal{S}$.

We prove Theorems 1.2 and 1.3 in Sections 4 and 5 . In particular, we describe the structure of the spaces $\overline{\mathcal{V}}_{1}^{(1)}(\mu)$ and $\overline{\mathcal{V}}_{2}^{(1,1)}(\mu)$ in Subsection 4.2 and conclude that they define a homology class in a compact oriented stratified topological orbifold. Since $\overline{\mathcal{S}}_{1}(\mu)$ is shown to be an oriented topological manifold in [17. Subsection 5.4], it follows that all the terms on the right-hand side of the formulas in the two propositions are well-defined. In Subsections 4.1 and 5.1 we write $\mathcal{V}_{1}^{(2)}(\mu)$ and $\mathcal{S}_{1}^{(1)}(\mu)$ in the form (1.5) and (1.3), respectively, and express the first term on the right-hand side in terms of evaluations of tautological classes on $\overline{\mathcal{V}}_{1}^{(1)}(\mu)$ and on related spaces. Lemmas 1.5] and 1.7 are proved in [17. The first statement of Lemma 1.8 is a special case of [20, Theorem 1.1]. The remaining statements of Lemma 1.8 and Lemmas 1.4 and 1.6 are proved in Section 6 . In Subsections 7.1 and 7.2, we show that our method recovers the formulas of 6 ] 
and [15] solving Questions $\mathrm{D}$ and $\mathrm{E}$, which are the $\mathbb{P}^{2}$ analogues of the problems addressed by Theorems 1.2 and 1.3. We conclude by proving Theorem 1.1 in Subsection 7.3 where we construct a tree of contributions and thus illustrate a point made at the end of Subsection 1.2

\subsection{General remarks}

Given the claims made in the abstract and at the end of Subsection 1.1, the reader may wonder why this paper is so long, why the notation is so involved, and why a more general case is not done. Doing a more general case, instead of the examples we work out, may in fact shorten this paper. However, the additional notation needed to describe a general case is likely to completely obscure the computational method presented here.

The topological part of our method consists of Propositions 2.18A and 2.18B. The somewhat involved notation of Subsection 2.2 formally states what it means to take the leading term(s) of a section along the normal direction to a submanifold. Proposition 3.5 gives power-series expansions for all relevant vector-bundle sections near all boundary strata of moduli spaces of stable rational maps. Describing the terms involved in the power-series expansions requires quite a bit of notation. However, as we will see in later sections, very few boundary strata actually matter in our computations, and the expansions of Proposition 3.5 corresponding to such strata are rather simple. In practice, it is best to draw a tree of these simple strata along with all the relevant topological data; then deriving formulas such as those of Theorems 1.2 and 1.3 becomes a nearlymechanical task.

The sections and linear maps between vector bundles that we introduce in Subsection 3.2 are described in an analytic way. Nevertheless, it is likely that the numbers of zeros of these and related linear maps have an algebraic interpretation, and that the same is true of our entire computational approach. Furthermore, there seem to be some general properties that remain to be explored. For example, finding any difference between our formulas for $\left|\mathcal{S}_{1}(\mu)\right|$ in Theorem 1.1 and for the genus-one correction term $C R_{1}(\mu)$ in [20, Theorem 1.1] requires a rather careful comparison of the two. One may also notice some similarity between the expressions for various level 1 numbers in Lemmas 1.4 1.8. A topological property of the number of zeros of an affine map in a simple case is described in [19, Corollary 4.7].

We conclude this introductory section by describing some classes of enumerative problems definitely and likely solvable by the method described in this paper. 
Suppose $\mathcal{C}$ is a one-component curve in $\mathbb{P}^{n}$, or in another algebraic manifold $M$. Let $u: \widetilde{\mathcal{C}} \longrightarrow \mathcal{C}$ be a normalization of $\mathcal{C}$, ie $\widetilde{\mathcal{C}}$ is a smooth connected complex curve and $u: \widetilde{\mathcal{C}} \longrightarrow M$ is a holomorphic map such that the image of $u$ is $\widetilde{\mathcal{C}}$ and $u$ is one-to-one outside of a finite set of points of $\widetilde{\mathcal{C}}$. If $\widetilde{p}$ is a point in $\widetilde{\mathcal{C}}$, let $\sigma_{u}(\widetilde{p})$ be the nonnegative integer such that the first $\sigma_{u}(\widetilde{p})$ derivatives of $u$ at $\widetilde{p}$ vanish, but the derivative of order $\sigma_{u}(\widetilde{p})+1$ does not vanish at $p$. For example, if $\sigma_{u}(\widetilde{p})=0,\left.d u\right|_{\tilde{p}} \neq 0$, ie $p$ is a smooth point of the branch of the curve $\mathcal{C}$ corresponding to $\widetilde{p}$. If $\sigma_{u}(\widetilde{p})=1$,

$$
\left.d u\right|_{\widetilde{p}}=0, \quad \text { but }\left.\quad \mathcal{D}_{\widetilde{p}}^{(2)} u \equiv \mathcal{D} d u\right|_{\tilde{p}} \neq 0,
$$

where $\mathcal{D}$ denotes the covariant differentiation with respect to some connection in $T M$. In other words, the branch of the curve $\mathcal{C}$ at $p$ corresponding to $\widetilde{p}$ has a cusp at $p$. If $p$ is a point of $\mathcal{C}$, we denote by $\sigma_{0}(p)$ the set of branches of $\mathcal{C}$ at $p$; in particular, $\left|\sigma_{0}(p)\right|=\left|u^{-1}(p)\right|$. For each $i \in \sigma_{0}(p)$, let $\sigma(p ; i)=\sigma_{u}\left(\widetilde{p}_{i}\right)$ if $\widetilde{p}_{i}$ is the point in $\widetilde{\mathcal{C}}$ such that $u$ maps a small neighborhood of $\widetilde{p}_{i}$ into the branch $i$ at $p$. Let $\sigma_{0}(p)=\bigsqcup \sigma_{0, k}(p)$ be the partition such that

$$
\mathcal{D}_{\widetilde{p}_{i}}^{\left(\sigma_{u}\left(\widetilde{p}_{i}\right)+1\right)} u \| \mathcal{D}_{\widetilde{p}_{j}}^{\left(\sigma_{u}\left(\widetilde{p}_{j}\right)+1\right)} u \quad \Longleftrightarrow \quad i, j \in \sigma_{0, k}(p) \text { for some } k .
$$

For example, if $\sigma_{0}(p)=\sigma_{0,1}(p)=\left\{\widetilde{p}_{1}, \widetilde{p}_{2}\right\}$ and $\sigma(p ; 1)=\sigma(p ; 2)=0$, the curve $\mathcal{C}$ has a tacnode at $p$. We take

$$
\underline{\sigma}(p)=\left(\sigma_{0}(p),\left\{\sigma_{0, k}(p)\right\},\{\sigma(p ; i)\}\right) .
$$

The infinite set $\{\underline{\sigma}(p): p \in \mathcal{C}\}$ describes the singularities of the curve $\mathcal{C}$. However, for all but finitely many points $p$ in $\mathcal{C}, \sigma_{0}(p)=\{i\}$ is a single-element set and $\sigma(p ; i)=0$. Thus, we say that the curve $\mathcal{C}$ has the set of singularities

$$
\{\underline{\sigma}(\alpha): \alpha=1, \ldots, N\}, \quad \text { where } \quad \underline{\sigma}(\alpha)=\left(\sigma_{0}(\alpha),\left\{\sigma_{0, k}(\alpha)\right\},\{\sigma(\alpha ; i)\}\right),
$$

if there are distinct points $p_{1}, \ldots, p_{N}$ of $\mathcal{C}$ such that for all $\alpha$ and some identification of $\sigma_{0}(\alpha)$ with the set of branches of $\mathcal{C}$ at $p_{\alpha}, \underline{\sigma}(\alpha)=\underline{\sigma}\left(p_{\alpha}\right)$. The method of this paper can be used to determine the number of one-component rational curves in a projective space that have any one-element set of singularities of the form $\{(\sigma,\{\sigma\},\{\sigma(i)\})\}$. In other words, the curves are to have one $|\sigma|$-fold singular point and the branches of the curves are to have cusps of the orders $\{\sigma(i)\}$. In particular, we are not imposing any tacnodal kind of condition. The singular point of the curves may be required to fall on a subvariety.

Some types of singularities that cause problems for this method are the flex, two nodes, two cusps, and the tacnode. The reason is that the expansions of bundle sections given in Propositions 3.5 are not sufficiently fine in the cases 
when the boundary stratum involves curves of very low, but positive, degree. For example, one of the strata of the space of one-component degree- $d$ rational curves with one marked point $y_{1}$ consists of two-component curves, one of which has degree one and carries the marked point $y_{1}$. Every element of this stratum has a flex at $y_{1}$, ie is a zero of a certain bundle section $s$. However, Proposition 3.5 does not give a sufficiently fine description of the behavior of $s$ near this stratum. On the other hand, a simple dimension-counting argument shows that such a boundary stratum cannot occur if the dimension $n$ of the projective space is two. If we would like to count one-component curves that have two cusps, or two nodes, or a tacnode, the problem stratum is the one consisting of two-component curves, one of which is a double line and carries two marked points. Again, a dimension-counting argument shows that this boundary stratum does not occur unless $n$ is at least 6 , 4, or 5, respectively. Indeed, among the examples worked out in this paper are the enumerations of tacnodal rational curves in $\mathbb{P}^{2}$ and $\mathbb{P}^{3}$.

Many types of curves with multiple components and with first-order tangency conditions to disjoint subvarieties can be counted as well. In fact, as the results described in Subsection 1.3 indicate, counting one-component singular curves involves counting multiple-components curves with simpler singularities. We plan to elaborate more on what types of curves can be counted and why in a later paper. Finally, due to the explicit nature of the gluing maps used, it should be possible to sharpen the expansions of Propositions 3.5 along the few problem strata that appear in more general cases. If so, every enumerative problem, in the sense described above, will be solvable.

Remark This paper concerns counting curves in $\mathbb{P}^{n}$, but our method may apply to counting curves with singularities in some other Kahler manifolds as well. Our aim is to express curve counts in terms of top intersections of tautological classes on moduli spaces of stable rational maps. Thus, in order to obtain actual numbers we make use of:

Fact 0 Top intersections of tautological classes on $\overline{\mathfrak{M}}_{0, k}\left(\mathbb{P}^{n}, d\right)$ are computable. It is essential for the method itself that the moduli spaces $\overline{\mathfrak{M}}_{0, k}\left(\mathbb{P}^{n}, d\right)$ have the expected structure. This is due to

Fact 1 If $u: \mathbb{P}^{1} \longrightarrow \mathbb{P}^{n}$ is a holomorphic map, then

$$
H^{1}\left(\mathbb{P}^{1} ; u^{*} T \mathbb{P}^{n} \otimes \mathcal{O}_{\mathbb{P}^{1}}(-1)\right)=0 .
$$

In order for our method to apply to a Kahler manifold $M$, Fact 1 needs to hold with $\mathbb{P}^{n}$ replaced by $M$ for maps up to the relevant "degree", ie the homology class of the curves to be counted. Additional positivity conditions, dependent 
on the type of singularities involved, need to be satisfied as well. For example, in order to count curves with a cusp in $\mathbb{P}^{n}$, we rely on

Fact 2 If $u: \mathbb{P}^{1} \longrightarrow \mathbb{P}^{n}$ is a nonconstant holomorphic map, then

$$
H^{1}\left(\mathbb{P}^{1} ; u^{*} T \mathbb{P}^{n} \otimes \mathcal{O}_{\mathbb{P}^{1}}(-2)\right)=0 .
$$

Theorem 1.1 is valid with $\mathbb{P}^{n}$ replaced by $M$ as long as Facts 1 and 2 hold with $\mathbb{P}^{n}$ replaced by $M$ for maps of degree up $d$.

\section{Topology}

\subsection{Monomials maps}

This section contains details of the topological aspects of the computational approach of this paper. We describe the setting in the next subsection and state and justify the reductive method for computing boundary contributions in Subsection 2.3. The present subsection collects a few basic facts that are used elsewhere in this section. The key statements here are Definition 2.1 and Propositions $2.3 \mathrm{~A}$ and $2.3 \mathrm{~B}$

We denote by $\overline{\mathbb{R}}^{+}$the set of nonnegative reals. Let $\beta: \overline{\mathbb{R}}^{+} \longrightarrow[0,1]$ be a smooth cutoff function such that

$$
\beta(t)=\left\{\begin{array}{ll}
0 & \text { if } t \leq 1, \\
1 & \text { if } t \geq 2 ;
\end{array} \quad \text { and } \quad \beta^{\prime}(t)>0 \text { if } t \in(1,2)\right.
$$

If $\delta>0$, let $\beta_{\delta} \in C^{\infty}\left(\overline{\mathbb{R}}^{+} ; \mathbb{R}\right)$ be given by $\beta_{\delta}(t)=\beta\left(\delta^{-\frac{1}{2}} t\right)$. We also denote by $\beta_{\delta}$ the natural extension of $\beta_{\delta}$ to $\mathbb{C}^{n}$ :

$$
\beta_{\delta}(\underline{z})=\beta_{\delta}(|\underline{z}|)
$$

where $|\underline{z}|=\sqrt{\left|z_{1}\right|^{2}+\ldots+\left|z_{n}\right|^{2}}$ if $\underline{z}=\left(z_{1}, \ldots, z_{n}\right) \in \mathbb{C}^{n}$. We write $B_{\delta}\left(0, \mathbb{C}^{n}\right)$ for the open ball of radius $\delta$ about 0 in $\mathbb{C}^{n}$. Let $Y_{n}$ be the union of the $n$ codimension-one coordinate subspaces $\mathbb{C}^{k} \times\{0\} \times \mathbb{C}^{n-1-k}$ in $\mathbb{C}^{n}$.

Definition 2.1 Suppose $m$ and $n$ are positive integers and $\mathcal{A}=(a)_{i j}$ is an $m \times n$ integer matrix. Then:

(1) A function $\rho: \mathbb{C}^{n}-Y_{n} \longrightarrow \mathbb{C}^{m}-Y_{m}$ is a degree- $\mathcal{A}$ monomials map if

$$
\rho\left(z_{1}, \ldots, z_{n}\right)=\left(z_{1}^{a_{1,1}} \ldots z_{n}^{a_{1, n}}, \ldots, z_{1}^{a_{m, 1}} \ldots z_{n}^{a_{m, n}}\right)
$$

for all $\left(z_{1}, \ldots, z_{n}\right) \in \mathbb{C}^{n}-Y_{n}$. 
(2) A degree- $\mathcal{A}$ monomials map $\rho$ is nondegenerate if $\operatorname{rk} \rho \equiv \operatorname{rk} \mathcal{A}=m$.

(3) If $m=n$, a nondegenerate degree $-\mathcal{A}$ monomials map $\rho$ is positive (or negative) if all components of the vector $\mathcal{A}^{-1} \underline{1}$ are positive (or negative).

(4) If $m=n$, a nondegenerate degree- $\mathcal{A}$ monomials map $\rho$ is neutral if it is neither positive nor negative.

In (3) above, $\underline{1}$ denotes the column vector of length $n$ consisting of all ones. If $m=n$, let $\operatorname{det} \rho$ denote the determinant of the square matrix $\mathcal{A}$.

Definition 2.2 Suppose $n$ is a positive integer, $\mathcal{A}$ is an $n \times n$ nondegenerate integer matrix, and $\mathcal{A}_{1}$ and $\mathcal{A}_{2}$ are row vectors of length $n$. Let $\rho, \rho_{1}$, and $\rho_{2}$ be monomials maps of degrees $\mathcal{A}, \mathcal{A}_{1}$, and $\mathcal{A}_{2}$, respectively.

(1) If $\rho$ is a positive monomials map, $\rho_{1}>_{\rho} \rho_{2}$ if $\mathcal{A}_{1} \mathcal{A}^{-1} \underline{1}<\mathcal{A}_{2} \mathcal{A}^{-1} \underline{1}$.

(2) If $\rho$ is a negative monomials map, $\rho_{1}>_{\rho} \rho_{2}$ if $\mathcal{A}_{1} \mathcal{A}^{-1} \underline{1}>\mathcal{A}_{2} \mathcal{A}^{-1} \underline{1}$.

Remark The notions of nondegenerate, positive, negative, and neutral of Definition 2.1 are invariant under every reordering of coordinates on the domain and/or the target space. The same is true of the partial-order relation introduced by Definition 2.2. We describe geometric consequences of these properties below.

Proposition 2.3A If $\rho: \mathbb{C}^{n}-Y_{n} \longrightarrow \mathbb{C}^{n}-Y_{n}$ is a degree- $\mathcal{A}$ neutral monomials map and $\mathcal{K}$ is a compact subset of $\mathbb{C}^{n}-Y_{n}$, there exists $\delta^{*}=\delta^{*}(\mathcal{A}, \mathcal{K}) \in \mathbb{R}^{+}$ such that

$$
\left(B_{\delta^{*}}\left(0, \mathbb{C}^{n}\right)-Y_{n}\right) \cap \rho^{-1}\left(\mathbb{R}^{+} \cdot \mathcal{K}\right)=\emptyset
$$

Proposition 2.3B Suppose $\rho: \mathbb{C}^{n}-Y_{n} \longrightarrow \mathbb{C}^{n}-Y_{n}$ is a degree- $\mathcal{A}$ positive (or negative) monomials map and $\mathcal{K}$ is a precompact open subset of $\mathbb{C}^{n}-Y_{n}$. Then:

(1) The set $\rho^{-1}\left(\mathbb{R}^{+} \cdot \overline{\mathcal{K}}\right)$ is closed in $\mathbb{C}^{n}-\{0\}$.

(2) For every $\delta^{*} \in \mathbb{R}^{+}, \delta \in\left(0, \delta^{*}\right)$, and $\delta_{+} \in\left(\delta^{1 / 2}, \infty\right)$, there exists

$$
\epsilon=\epsilon\left(\mathcal{A}, \delta^{*}, \delta, \delta_{+}, \mathcal{K}\right) \in \mathbb{R}^{+}
$$

such that for all $t \in(0, \epsilon)$, the map

$$
t^{-1} \beta_{\delta} \rho:\left(B_{2 \delta^{* 1 / 2}}\left(0, \mathbb{C}^{n}\right)-Y_{n}\right) \cap\left\{t^{-1} \beta_{\delta} \rho\right\}^{-1}(\mathcal{K}) \longrightarrow \mathcal{K}
$$


is a smooth covering projection of oriented order $|\operatorname{det} \rho|(-|\operatorname{det} \rho|)$. Furthermore,

$$
\left(B_{2 \delta^{* 1 / 2}}\left(0, \mathbb{C}^{n}\right)-Y_{n}\right) \cap\left\{t^{-1} \beta_{\delta} \rho\right\}^{-1}(\mathcal{K}) \subset B_{\delta_{+}}\left(0, \mathbb{C}^{n}\right) .
$$

(3) If $\rho_{1}$ and $\rho_{2}$ are monomials maps of degrees $\mathcal{A}_{1}$ and $\mathcal{A}_{2}$ such that $\rho_{1}>_{\rho} \rho_{2}$, for every $\epsilon \in \mathbb{R}^{+}$, there exists $\delta=\delta\left(\mathcal{A}, \mathcal{A}_{1}, \mathcal{A}_{2}, \epsilon\right)$ such that $\left|\rho_{2}(\underline{z})\right| \leq \epsilon\left|\rho_{1}(\underline{z})\right|$ for all $\underline{z} \in\left(B_{\delta}\left(0, \mathbb{C}^{n}\right)-Y_{n}\right) \cap \rho^{-1}\left(\mathbb{R}^{+} \cdot \mathcal{K}\right)$.

The rest of this subsection is devoted to proving these propositions. Note that

$$
\left.\operatorname{det} D \rho\right|_{\left(z_{1}, \ldots, z_{n}\right)}=(\operatorname{det} \rho) z_{1}^{A_{1}-1} \ldots z_{n}^{A_{n}-1},
$$

where $A_{j}=\sum_{i=1}^{i=n} a_{i, j}$. Thus, $\operatorname{Im} \rho$ contains an open subset of $\mathbb{C}^{n}$ if (and only if) $\rho$ is nondegenerate in the sense of (2) of Definition 2.1. Since $\rho$ is a rational function in complex variables, it follows that $\operatorname{Im} \rho$ is a dense open subset of $\mathbb{C}^{n}$ if $\rho$ is nondegenerate. Since $\rho$ is given by monomials, $\operatorname{Im} \rho$ is in fact all of $\mathbb{C}^{n}-Y_{n}$. Thus

$$
\rho: \mathbb{C}^{n}-Y_{n} \longrightarrow \mathbb{C}^{n}-Y_{n}
$$

is a local diffeomorphism. By Lemma 2.8, this map is in fact a covering projection of order $|\operatorname{det} \rho|$.

Remark The proof of Lemma 2.8 does not rely on Lemmas 2.4 2.6 or Corollary 2.7. We postpone its proof until the very end of this subsection in order to focus on the main aspects of the proof of Propositions $2.3 \mathrm{~A}$ and $2.3 \mathrm{~B}$.

Denote by $\tilde{Y}_{n}$ the union of all the $n$ codimension-one coordinate subspaces $\mathbb{R}^{k} \times\{0\} \times \mathbb{R}^{n-1-k}$ in $\mathbb{R}^{n}$. Let $\tilde{Y}_{n}^{*}=\widetilde{Y}_{n} \cap S^{n-1}$. We identify $\mathbb{C}^{n}-Y_{n}$ with

$$
\mathbb{R}^{+} \times\left(S^{n-1}-\tilde{Y}_{n}^{*}\right) \times\left(S^{1}\right)^{n}
$$

by the map

$$
\begin{aligned}
\mathbb{C}^{n}-Y_{n} & \longrightarrow \mathbb{R}^{+} \times\left(S^{n-1}-\tilde{Y}_{n}^{*}\right) \times\left(S^{1}\right)^{n} \\
\underline{z}=\left(z_{1}, \ldots, z_{n}\right) & \longmapsto\left(|\underline{z}|, \frac{\left(\left|z_{1}\right|, \ldots,\left|z_{n}\right|\right)}{|\underline{z}|},\left(\frac{z_{1}}{\left|z_{1}\right|}, \ldots, \frac{z_{n}}{\left|z_{n}\right|}\right)\right) .
\end{aligned}
$$

With respect to this decomposition,

$$
\rho(r, \varphi, \theta)=(f(r, \varphi), g(r, \varphi), h(\theta)),
$$

where $(f(\underline{r}), g(\underline{r}))=\rho(\underline{r}) \in \mathbb{R}^{n}$ and

$$
h\left(e^{i \theta_{1}}, \ldots, e^{i \theta_{n}}\right)=\rho\left(e^{i \theta_{1}}, \ldots, e^{i \theta_{n}}\right) \in\left(S^{1}\right)^{n} \subset \mathbb{C}^{n} .
$$

Proposition $2.3 \mathrm{~A}$ follows immediately from: 
Lemma 2.4 If $\rho$ is a neutral monomials map, for every compact subset $\mathcal{K}$ of $S^{n-1}-\widetilde{Y}_{n}^{*}$, there exists $\delta \in \mathbb{R}^{+}$such that for all $r \in(0, \delta),\{g(r, \cdot)\}^{-1}(\mathcal{K})=\emptyset$.

Proof We use the variables $s_{1}, \ldots, s_{n}$ to denote the standard Euclidean coordinates on the target space $\mathbb{R}^{n}$, as well as the corresponding component functions of $(f, g)$. Let

$$
\widetilde{\mathcal{A}}=\left(\begin{array}{ccc}
a_{2,1}-a_{1,1} & \ldots & a_{2, n}-a_{1, n} \\
\vdots & & \vdots \\
a_{n, 1}-a_{1,1} & \ldots & a_{n, n}-a_{1, n}
\end{array}\right)
$$

It is sufficient to show that, for some $i=2, \ldots, n$,

$$
\limsup _{r \rightarrow 0}\left\{\frac{s_{i}(\underline{r})}{s_{1}(\underline{r})}:|\underline{r}|<r\right\}=0 \quad \text { or } \quad \liminf _{r \rightarrow 0}\left\{\frac{s_{i}(\underline{r})}{s_{1}(\underline{r})}:|\underline{r}|<r\right\}=\infty .
$$

This condition is equivalent to

$$
\begin{gathered}
\limsup _{t \rightarrow-\infty}\left\{ \pm \sum_{j=1}^{n}\left(a_{i, j}-a_{1, j}\right) t_{j}: t_{j} \in(-\infty, t)\right\}=-\infty \\
\Longleftrightarrow\left\{\begin{aligned}
\quad a_{i, j}-a_{1, j} \geq 0 \text { for all } j=1, \ldots, n \\
\text { or } \quad a_{i, j}-a_{1, j} \leq 0 \text { for all } j=1, \ldots, n .
\end{aligned}\right.
\end{gathered}
$$

Note that the two lines above are equivalent because $\mathcal{A}$ is assumed to be nondegenerate. If (2.2) is not satisfied by any $i=2, \ldots, n$, for every nonzero vector $\underline{x} \in \mathbb{R}^{n-1}$ there exists a vector $\underline{c} \in \mathbb{R}^{n}$ such that

$$
\underline{x}^{t} \tilde{\mathcal{A}} \underline{c}>0 \quad \text { and } \quad c_{1}, \ldots, c_{n} \geq 0 .
$$

This means that the image of $\widetilde{\mathcal{A}}^{t}$ contains no nonzero vector with all components of the same sign. Thus

there exists $\left(x_{1}, \ldots, x_{n}\right)$ in $\operatorname{ker} \widetilde{\mathcal{A}}-\{0\}$ such that $x_{1}, \ldots, x_{n}>0$.

Let $\widetilde{\mathcal{A}}_{\hat{j}}$ be the matrix obtained from $\widetilde{\mathcal{A}}$ by removing the $j$ th column. Since $\rho$ is nondegenerate, $\operatorname{det} \widetilde{\mathcal{A}}_{\hat{j}} \neq 0$ for some $j$. Then, by Cramer's Rule,

$$
x_{j^{\prime}}=\frac{(-1)^{j+j^{\prime}-1} \operatorname{det} \widetilde{\mathcal{A}}_{\widehat{j}^{\prime}}}{\operatorname{det} \widetilde{\mathcal{A}}_{\hat{j}}}\left(-x_{j}\right)=\frac{(-1)^{j^{\prime}} \operatorname{det} \widetilde{\mathcal{A}}_{\widehat{j}^{\prime}}}{(-1)^{j} \operatorname{det} \widetilde{\mathcal{A}}_{\hat{j}}} x_{j} \text { for all } j^{\prime} .
$$

Since $\rho$ is neutral,

$$
\frac{(-1)^{j^{\prime}} \operatorname{det} \widetilde{\mathcal{A}}_{\widehat{j}^{\prime}}}{(-1)^{j} \operatorname{det} \widetilde{\mathcal{A}}_{\widehat{j}}} \leq 0
$$

for some $j^{\prime}$. Thus (2.4) contradicts (2.3). 
Lemma 2.5 If $\rho$ is a positive or negative monomials map, then for every $r \in \mathbb{R}^{+}$, the map

$$
g(r, \cdot): S^{n-1}-\tilde{Y}_{n}^{*} \longrightarrow S^{n-1}-\tilde{Y}_{n}^{*}, \text { given by } \theta \longmapsto g(r, \theta),
$$

is a local diffeomorphism.

Proof We assume that $n \geq 2$; otherwise, there is nothing to prove. Suppose $g(r, \cdot)$ is not a local diffeomorphism at $\underline{r}=\left(r_{1}, \ldots, r_{n}\right) \in \mathbb{R}^{n}-\widetilde{Y}_{n}$. Then, there exists $\underline{c} \in \mathbb{R}^{n}-\{0\}$ such that

$$
\sum_{j=1}^{n} r_{j} c_{j}=0 \text { and } \sum_{j=1}^{n}\left(a_{i, j}-a_{1, j}\right) r_{j}^{-1} c_{j}=0 \text { for all } i=2, \ldots, n \text {. }
$$

The first equation above is equivalent to the condition $\underline{c} \in T_{\underline{r}} S^{n-1}$. The second equation means that the ratio of the $i$ th and the first Euclidean components of the function $(f, g)$ does not change in the direction of $\underline{c}$ at $\underline{r}$. The $n$ conditions (2.5) are equivalent to $\mathcal{A}^{(1)}\left(\underline{r}^{2}\right) \underline{c}^{\prime}=0 \in \mathbb{R}^{n}$, where:

$$
\mathcal{A}^{(1)}\left(\underline{r}^{2}\right) \equiv \mathcal{A}^{(1)}\left(r_{1}^{2}, \ldots, r_{n}^{2}\right)=\left(\begin{array}{ccc}
r_{1}^{2} & \ldots & r_{n}^{2} \\
a_{2,1}-a_{1,1} & \ldots & a_{2, n}-a_{1, n} \\
\vdots & & \vdots \\
a_{n, 1}-a_{1,1} & \ldots & a_{n, n}-a_{1, n}
\end{array}\right)
$$

This equation has a nonzero solution only if $\operatorname{det} \mathcal{A}^{(1)}\left(\underline{r}^{2}\right)=0$. However,

$$
\operatorname{det} \mathcal{A}^{(1)}\left(\underline{r}^{2}\right)=\sum_{j=1}^{n}(-1)^{j-1}\left(\operatorname{det} \widetilde{\mathcal{A}}_{\hat{j}}\right) r_{j}^{2} \text {. }
$$

Since $\rho$ is positive or negative, all the elements of the set $\left\{(-1)^{j-1} \operatorname{det} \widetilde{\mathcal{A}}_{\hat{j}}\right\}$ have the same sign. Thus, $\operatorname{det} \mathcal{A}^{(1)}\left(\underline{r}^{2}\right)$ does not vanish on $\mathbb{R}^{n}-\{0\}$. It follows that the differential of $g(r, \cdot)$ is an isomorphism everywhere on $S^{n-1}-\widetilde{Y}_{n}^{*}$.

Let $Y_{n}^{*}=Y_{n} \cap S^{2 n-1} \subset \mathbb{C}^{n}$. We identify $\mathbb{C}^{n}-Y_{n}$ with $\mathbb{R}^{+} \times\left(S^{2 n-1}-Y_{n}^{*}\right)$ and denote by

$$
(\tilde{f}, \widetilde{g}): \mathbb{R}^{+} \times\left(S^{2 n-1}-Y_{n}^{*}\right) \longrightarrow \mathbb{R}^{+} \times\left(S^{2 n-1}-Y_{n}^{*}\right)
$$

the pair of maps corresponding to $\rho$.

Lemma 2.6 If $\rho$ is a positive (or negative) monomials map, then for every $r \in \mathbb{R}^{+}$, the map

$$
\widetilde{g}(r, \cdot): S^{2 n-1}-Y_{n}^{*} \longrightarrow S^{2 n-1}-Y_{n}^{*}
$$

is a local orientation-preserving (or orientation-reversing) diffeomorphism, and is a covering projection of oriented order $|\operatorname{det} \rho|($ or $-|\operatorname{det} \rho|)$. 
Proof To prove the first claim, we assume that $\rho$ is a positive monomials map; the negative case is proved similarly. By Lemma 2.5 and the decomposition $\widetilde{g}=(g, h)$, it follows that $\widetilde{g}(r, \cdot)$ is a local diffeomorphism. Since $\rho$ is orientationpreserving everywhere, $\widetilde{g}(r, \cdot)$ is orientation-preserving at $(r, \theta)$ if

$$
\left\langle\left\{\left.D \rho\right|_{(r, \theta)}\right\}^{-1}\left(\frac{\partial}{\partial r}\right),\left(\frac{\partial}{\partial r}\right)\right\rangle>0 .
$$

Let $\underline{c} \in \mathbb{R}^{n}$ be given by $\mathcal{A} \underline{c}=\underline{1} \in \mathbb{R}^{n}$. Then

$$
\rho\left(t^{c_{1}} z_{1}, \ldots, t^{c_{n}} z_{n}\right)=t \rho\left(z_{1}, \ldots, z_{n}\right) \quad \text { and }\left.\quad \frac{d}{d t} t \rho(z)\right|_{t=1}=\frac{\partial}{\partial r} .
$$

Since $\rho$ is positive, $c_{1}, \ldots, c_{n} \in \mathbb{R}^{+}$and thus

$$
\frac{d}{d t}\left|\left(t^{c_{1}} z_{1}, \ldots, t^{c_{n}} z_{n}\right)\right|>0 .
$$

The desired inequality (2.6) is immediate from (2.7) and (2.8). The remaining claim follows from Lemma 2.8 and (2.7), since the curves

$$
t \longmapsto\left(t^{c_{1}} z_{1}, \ldots, t^{c_{n}} z_{n}\right) \text { and } t \longmapsto\left(t w_{1}, \ldots, t w_{n}\right) \text {, for } t \in(0, \infty),
$$

with $\left(z_{1}, \ldots, z_{n}\right)$ and $\left(w_{1}, \ldots, w_{n}\right) \in S^{2 n-1}$, foliate $\mathbb{C}^{n}-Y_{n}$ and intersect each $(2 n-1)$-sphere $r=$ const exactly once.

Remark The first claim of Proposition 2.3B now follows from Lemma 2.8, the first identity in (2.7), and the assumptions that all the exponents $c_{i}$ have the same sign.

Corollary 2.7 Suppose $\rho$ is a positive (or negative) monomials map, $\delta^{*} \in \mathbb{R}^{+}$, $\delta \in\left(0, \delta^{*}\right)$, and $\mathcal{K}$ is a precompact open subset of $\mathbb{C}^{n}-Y_{n}$. Then, there exists $\epsilon \in \mathbb{R}^{+}$such that for all $t \in(0, \epsilon)$, the map

$$
t^{-1} \beta_{\delta} \rho:\left(B_{2 \delta^{* 1 / 2}}\left(0, \mathbb{C}^{n}\right)-Y_{n}\right) \cap\left\{t^{-1} \beta_{\delta} \rho\right\}^{-1}(\mathcal{K}) \longrightarrow \mathcal{K}
$$

is a smooth covering projection of oriented order $|\operatorname{det} \rho|(-|\operatorname{det} \rho|)$.

Proof We prove this corollary in the case $\rho$ is negative. If $\rho$ is positive, a stronger claim can be obtained by a similar and somewhat simpler argument. With notation as above, $\beta_{\delta} \rho$ corresponds to the pair $\left(\beta_{\delta} \widetilde{f}, \widetilde{g}\right)$ with respect to the splitting of $\mathbb{C}^{n}-Y_{n}=\mathbb{R}^{+} \times\left(S^{2 n-1}-Y_{n}^{*}\right)$. Thus

$$
\left.\operatorname{det} D\left(\beta_{\delta} \rho\right)\right|_{(r, \theta)}=\left.\beta_{\delta}(r) \operatorname{det} D \rho\right|_{(r, \theta)}+\left.\beta_{\delta}^{\prime}(r) \widetilde{f}(r, \theta) \operatorname{det}\left(\frac{\partial \widetilde{g}}{\partial \theta}\right)\right|_{(r, \theta)} \text {. }
$$


Let $\widetilde{\mathcal{K}} \subset S^{2 n-1}-Y_{n}^{*}$ be the image of $\mathcal{K}$ under the projection map onto the second component. Since the closure of $\mathcal{K}$ in $\mathbb{C}^{n}-Y_{n}$ is compact, Lemma 2.6] implies that the set

$$
\widetilde{U} \equiv \bigcup_{\delta^{1 / 2} \leq r \leq 2 \delta^{* 1 / 2}}\{\widetilde{g}(r, \cdot)\}^{-1}(\widetilde{\mathcal{K}})
$$

has compact closure in $S^{2 n-1}-Y_{n}^{*}$. Thus, there exists $C>0$ such that

$$
\left.\operatorname{det} D \rho\right|_{(r, \theta)}<C \quad \text { and }\left.\quad \widetilde{f}(r, \theta) \operatorname{det}\left(\frac{\partial \widetilde{g}}{\partial \theta}\right)\right|_{(r, \theta)}<-C^{-1}
$$

for all $(r, \theta) \in\left(\delta^{1 / 2}, 2 \delta^{* 1 / 2}\right) \times \widetilde{U}$. The second bound is obtained by using Lemma 2.6. Choose $\eta>0$ such that

$$
\beta_{\delta}^{\prime}(r)>2 C^{2} \beta_{\delta}(r) \quad \text { for all } r \in\left(\delta^{1 / 2}, \delta^{1 / 2}+\eta\right) .
$$

Note that combining (2.9)-(2.10), we obtain

$$
\left.\operatorname{det} D\left(\beta_{\delta} \rho\right)\right|_{(r, \theta)}<0
$$

for all $(r, \theta) \in\left(\delta^{1 / 2}, \delta^{1 / 2}+\eta\right) \times \widetilde{U}$. Let $\epsilon>0$ be such that

$$
\epsilon \cdot \max \{|w|: w \in \mathcal{K}\}<\frac{1}{2} \min \left\{\beta_{\delta}(|\underline{z}|)|\rho(\underline{z})|: \underline{z} \in\left(\delta^{1 / 2}+\frac{1}{2} \eta, 2 \delta^{* 1 / 2}\right) \times \widetilde{U}\right\} .
$$

We claim that $\epsilon$ satisfies the required properties. Suppose that $t<\epsilon$, that $\underline{z}=(r, \theta) \in B_{2 \delta^{* 1 / 2}}\left(0, \mathbb{C}^{n}\right)$, and that $\left\{t^{-1} \beta_{\delta} \rho\right\}(z) \in \mathcal{K}$. Then

$$
r \in\left(\delta^{1 / 2}, 2 \delta^{* 1 / 2}\right) \Longrightarrow \theta \in\{\widetilde{g}(r, \cdot)\}^{-1}(\mathcal{K}) \subset \widetilde{U}
$$

Assumption (2.12) on $\epsilon$ then implies that $(r, \theta) \in\left(\delta^{1 / 2}, \delta^{1 / 2}+\frac{1}{2} \eta\right) \times \widetilde{U}$. From (2.11), we conclude that the determinant of the derivative of $t^{-1} \beta_{\delta} \rho$ at $\underline{z}$ is negative and the map

$$
t^{-1} \beta_{\delta} \rho:\left(B_{2 \delta^{* 1 / 2}}\left(0, \mathbb{C}^{n}\right)-Y_{n}\right) \cap\left\{t^{-1} \beta_{\delta} \rho\right\}^{-1}(\mathcal{K}) \longrightarrow \mathcal{K}
$$

is a local orientation-reversing diffeomorphism. It remains to see that for each point $(s, \vartheta) \in \mathcal{K}$,

$$
\left|\left\{(r, \theta) \in\left(\delta^{1 / 2}, \delta^{1 / 2}+\frac{1}{2} \eta\right) \times \widetilde{U}: \beta_{\delta}(r) \widetilde{f}(r, \theta)=t s, \widetilde{g}(r, \theta)=\vartheta\right\}\right|=|\operatorname{det} \rho| .
$$

By Lemma 2.6, there are smooth paths

$$
\theta_{i}:\left[\delta^{1 / 2}, \delta^{1 / 2}+\frac{1}{2} \eta\right] \longrightarrow \widetilde{U} \text { for } i=1, \ldots,|\operatorname{det} \rho|
$$

such that

$$
\{\widetilde{g}(r, \cdot)\}^{-1}(\vartheta)=\left\{\theta_{i}(r): i=1, \ldots,|\operatorname{det} \rho|\right\} \quad \text { and } \quad \theta_{i}(r) \neq \theta_{j}(r)
$$


for all $r \in\left[\delta^{1 / 2}, \delta^{1 / 2}+\frac{1}{2} \eta\right]$ and $i \neq j$. By (2.11) and (2.12),

$$
\begin{gathered}
\left\{\beta_{\delta} \widetilde{f}\right\}\left(\delta^{1 / 2}, \theta_{i}\left(\delta^{1 / 2}\right)\right)=0, \quad\left\{\beta_{\delta} \widetilde{f}\right\}\left(\delta^{1 / 2}+\frac{1}{2} \eta, \theta_{i}\left(\delta^{1 / 2}+\frac{1}{2} \eta\right)\right)>t s, \\
\text { and } \frac{d}{d r}\left\{\beta_{\delta} \widetilde{f}\right\}\left(r, \theta_{i}(r)\right)>0 \quad \text { for all } r \in\left(\delta^{1 / 2}, \delta^{1 / 2}+\frac{1}{2} \eta\right) .
\end{gathered}
$$

Thus, for each $i=1, \ldots,|\operatorname{det} \rho|$, there exists a unique number

$$
r_{i} \in\left(\delta^{1 / 2}, \delta^{1 / 2}+\frac{1}{2} \eta\right) \text { such that }\left\{\beta_{\delta} \tilde{f}\right\}\left(r_{i}, \theta_{i}\left(r_{i}\right)\right)=t s,
$$

as required.

Corollary 2.7 essentially concludes the proof of part (2) of Proposition 2.3B The claimed inclusion is achieved if, in the proof of Corollary 2.7. $\eta$ is chosen so that $\delta^{1 / 2}+\eta<\delta_{+}$.

We next prove part (3) of Proposition 2.3B Suppose $\mathcal{A}$ is a positive monomials map and $\widetilde{\mathcal{K}}=\rho^{-1}(\overline{\mathcal{K}})$. Since $\widetilde{\mathcal{K}}$ is a compact subset of $\mathbb{C}^{n}-Y_{n}$, there exists $r>0$ such that $B_{r}\left(0, \mathbb{C}^{n}\right) \cap \widetilde{\mathcal{K}}=\emptyset$. On the other hand, by Lemma 2.8 and the first identity in (2.7), if $t \in \mathbb{R}^{+}$and $\rho(\underline{z}) \in t \cdot \mathcal{K}$,

$$
\begin{gathered}
\left(z_{1}, \ldots, z_{n}\right)=\left(t^{c_{1}} w_{1}, \ldots, t^{c_{n}} w_{n}\right) \quad \text { for some }\left(w_{1}, \ldots, w_{n}\right) \in \rho^{-1}(K) \\
\Longrightarrow \quad|\underline{z}| \geq t^{\min c_{i}} r .
\end{gathered}
$$

Thus, if $|\underline{z}| \leq \delta$

$$
\left|\frac{\rho_{2}(\underline{z})}{\rho_{1}(\underline{z})}\right|=t^{\mathcal{A}_{2} \cdot \underline{c}-\mathcal{A}_{1} \cdot \underline{c}}\left|\frac{\rho_{2}(\underline{w})}{\rho_{1}(\underline{w})}\right| \leq C\left(\frac{\delta}{r}\right)^{\left(\mathcal{A}_{2} \cdot \underline{c}-\mathcal{A}_{1} \cdot \underline{c}\right) / \min c_{i}} .
$$

Since $\mathcal{A}_{2} \cdot \underline{c}>\mathcal{A}_{1} \cdot \underline{c}$ and $\min c_{i}>0$, the right-hand side above tends to zero with $\delta$. If $\rho$ is negative, the proof is similar.

Lemma 2.8 If $\rho: \mathbb{C}^{n}-Y_{n} \longrightarrow \mathbb{C}^{n}-Y_{n}$ is a nondegenerate monomials map, $\rho$ is a covering projection of order $|\operatorname{det} \rho|$.

Proof By (2.1), we only need to compute the order of the cover. We can view $\rho$ as a rational map from $\left(\mathbb{P}^{1}\right)^{N}$ to $\left(\mathbb{P}^{1}\right)^{N}$. In turn, this rational map induces a holomorphic map $\widetilde{\rho}: \widetilde{M} \longrightarrow\left(\mathbb{P}^{1}\right)^{N}$, where $\widetilde{M}$ is a compact complex manifold obtained from $\left(\mathbb{P}^{1}\right)^{N}$ by a sequence of blowups along submanifolds disjoint from $\mathbb{C}^{n}-Y_{n}$. Then

$$
\begin{aligned}
\operatorname{ord} \rho=\left\langle\widetilde{\rho}^{*}\left(\omega_{1} \wedge \ldots \wedge \omega_{n}\right),[\widetilde{M}]\right\rangle & =\int_{\widetilde{M}} \widetilde{\rho}^{*}\left(\omega_{1} \wedge \ldots \wedge \omega_{n}\right) \\
& =\int_{C^{n}-Y_{n}} \rho^{*}\left(\omega_{1} \wedge \ldots \wedge \omega_{n}\right),
\end{aligned}
$$


where $\omega_{i}$ is the Fubini-Study symplectic form on the $i$ th $\mathbb{P}^{1}$-factor of the target space. Since $\omega_{i}=\frac{i}{2 \pi} \frac{d z_{i} \wedge d \bar{z}_{i}}{\left(1+\left|z_{i}\right|^{2}\right)^{2}}$, see [4, p31], by (2.1),

$$
\begin{aligned}
\rho^{*}\left(\omega_{1}\right. & \left.\wedge \ldots \wedge \omega_{n}\right) \\
& =|\operatorname{det} \rho|^{2}\left(\frac{\mathfrak{i}}{2 \pi}\right)^{n} \frac{r_{1}^{2 A_{1}-2} \ldots r_{1}^{2 A_{n}-2} d z_{1} \wedge d \bar{z}_{1} \wedge \ldots \wedge d z_{n} \wedge d \bar{z}_{n}}{\left(1+r_{1}^{2 a_{1,1}} \ldots r_{n}^{2 a_{1, n}}\right)^{2} \ldots\left(1+r_{1}^{2 a_{n, 1}} \ldots r_{n}^{2 a_{n, n}}\right)^{2}} .
\end{aligned}
$$

Combining (2.13) and (2.14) and switching to polar coordinates, we obtain

$$
\operatorname{ord} \rho=2^{n}|\operatorname{det} \rho|^{2} \int_{0}^{\infty} \ldots \int_{0}^{\infty} \frac{r_{1}^{2 A_{1}-1} \ldots r_{1}^{2 A_{n}-1} d r_{1} \ldots d r_{n}}{\left(1+r_{1}^{2 a_{1,1}} \ldots r_{n}^{2 a_{1, n}}\right)^{2} \ldots\left(1+r_{1}^{2 a_{n, 1}} \ldots r_{n}^{2 a_{n, n}}\right)^{2}}
$$

The change of variables,

$$
\left(r_{1}, \ldots, r_{n}\right) \longrightarrow\left(r_{1}^{2 a_{1,1}} \ldots r_{n}^{2 a_{1, n}}, \ldots, r_{1}^{2 a_{n, 1}} \ldots r_{n}^{2 a_{n, n}}\right),
$$

reduces (2.15) to

$$
\begin{aligned}
\operatorname{ord} \rho & =|\operatorname{det} \rho| \int_{0}^{\infty} \ldots \int_{0}^{\infty} \frac{d r_{1} \ldots d r_{n}}{\left(1+r_{1}\right)^{2} \ldots\left(1+r_{n}\right)^{2}} \\
& =|\operatorname{det} \rho|\left(\int_{0}^{\infty} \frac{d r}{(1+r)^{2}}\right)^{n}=|\operatorname{det} \rho|,
\end{aligned}
$$

as claimed.

\subsection{Topological setup}

In this subsection, we give formal definitions of the topological objects to which the computational method described in the next subsection applies.

We start by extending the concept of monomials maps to vector bundles. All vector bundles we encounter will be assumed to be complex and normed. Vector bundles over smooth manifolds will in addition be smooth. Given a vector bundle $F \longrightarrow X$ and any map $\delta: X \longrightarrow \mathbb{R}$, put

$$
F_{\delta}=\left\{(b ; v) \in F:|v|_{b}<\delta(b)\right\} .
$$

If $F=\bigoplus_{i \in I} F_{i}$ is the direct sum of nontrivial subbundles and $I_{0} \subset I$, let

$$
Y\left(F ; I_{0}\right)=\bigcup_{i \in I_{0}}\left(\bigoplus_{j \in I-\{i\}} F_{j}\right) \subset F .
$$


Definition 2.9 Suppose $I_{0}, I$, and $J$ are finite sets, and $\mathcal{A}=\left(a_{i j}\right)$ is an integer-valued function on $\left(I_{0} \sqcup I\right) \times\left(I_{0} \sqcup J\right)$ such that for all $j \in I_{0}, a_{i j}=0$ if $i \neq j$ and $a_{i j}=1$ if $i=j$.

(1) Suppose $F_{j} \longrightarrow \mathcal{M}$ is a vector bundle for each $j \in I_{0}$ and a line bundle for each $j \in J$,

$$
F=\bigoplus_{j \in I_{0} \sqcup J} F_{j}, \quad \widetilde{F}_{i}=\bigotimes_{j \in I_{0} \sqcup J} F_{j}^{\otimes a_{i j}} \text { for all } i \in I_{0} \sqcup I, \quad \text { and } \quad \widetilde{F}=\bigoplus_{i \in I_{0} \sqcup I} \widetilde{F}_{i} .
$$

A function $\rho: F-Y(F ; J) \longrightarrow \widetilde{F}$ is a degree- $\mathcal{A}$ monomials map on $F$ if

$$
\pi_{i} \rho\left(\left(v_{j}\right)_{j \in I_{0} \sqcup J}\right)=\bigotimes_{j \in I_{0} \sqcup J} v_{j}^{\otimes a_{i, j}}
$$

for all $\left(v_{j}\right)_{j \in I_{0} \sqcup J} \in F-Y(F ; J)$ and $i \in I_{0} \sqcup I$, where $\pi_{i}: \widetilde{F} \longrightarrow \widetilde{F}_{i}$ is the projection map.

(2) Suppose $\rho$ is as in (1), $E_{i} \longrightarrow \mathcal{M}$ is a vector bundle for each $i \in I$,

$$
E=\bigoplus_{i \in I} E_{i}, \quad \text { and } \quad \widetilde{E}=\bigoplus_{i \in I} E_{i} \otimes \widetilde{F}_{i} .
$$

A function

$$
\widetilde{\rho}: E \oplus F-Y(E \oplus F ; J) \longrightarrow \widetilde{E}
$$

is a degree- $\mathcal{A}$ monomials map on $E \oplus F$ if

$$
\pi_{i} \widetilde{\rho}\left(\left(w_{i}\right)_{i \in I},(v)_{j \in I_{0} \sqcup J}\right)=w_{i} \otimes \pi_{i} \rho(v)
$$

for all $\left(\left(w_{i}\right)_{i \in I},(v)_{j \in I_{0} \sqcup J}\right) \in E \oplus F-Y(E \oplus F ; J)$ and $i \in I$.

A monomials map between vector bundles in the sense of Definition 2.9 part (1) can be viewed as a pair of bundle maps

$$
\rho_{I, J}: F_{J} \equiv \bigoplus_{j \in J} F_{j} \longrightarrow \widetilde{F}_{I} \equiv \bigoplus_{i \in I} \widetilde{F}_{i} \quad \text { and } \quad \rho_{I_{0}}: F_{J} \oplus \bigoplus_{i \in I_{0}} F_{i} \longrightarrow \bigoplus_{i \in I_{0}} \widetilde{F}_{i} \text {. }
$$

The vector bundles $F_{J}$ and $\widetilde{F}_{I}$ are sums of line bundles and the restriction of the bundle map $\rho_{I, J}$ to each fiber is a monomials map in the sense of Definition 2.1. The degree of this map is $\mathcal{A} \mid I \times J$. The bundle map $\rho_{I_{0}}$ has $I_{0}$ components, indexed by $i \in I$ :

$\pi_{i} \rho: F_{i} \oplus F_{J} \longrightarrow \widetilde{F}_{i} \equiv F_{i} \otimes \bigotimes_{j \in J} F_{j}^{\otimes a_{i j}}, \quad$ given by $\left(v_{i},\left(v_{j}\right)_{j \in J}\right) \longmapsto v_{i} \otimes \bigotimes_{j \in J} v_{j}$

A monomials map in the sense of Definition 2.9 part (2) is equivalent to a monomials map in the sense of Definition 2.9 part (1) with $\mathcal{A}$ being a function on $(I \sqcup \emptyset) \times(I \sqcup J)$. 
If $\rho$ and $\widetilde{\rho}$ are as in Definition 2.9 part (2), and $i \in I$, we put

$$
\widetilde{F}_{i}(\widetilde{\rho})=\widetilde{F}_{i} \quad \text { and } \quad \widetilde{\rho}_{i}(v)=\pi_{i} \rho(v) \in \widetilde{F}_{i}(\widetilde{\rho}) \text { for all } v \in F-Y(F ; J) .
$$

If $\rho$ is as in Definition 2.9part (1), we call $\rho$ nondegenerate if the restriction of $\rho$ to a fiber of $F$ is nondegenerate in the sense of Definition 2.1. for some choice of identifications of the sets $I_{0} \sqcup I$ and $I_{0} \sqcup J$ with the sets of integers $1, \ldots,\left|I_{0} \sqcup I\right|$ and $1, \ldots,\left|I_{0} \sqcup J\right|$. If $\rho$ is nondegenerate and $|I|=|J|$, we call $\rho$ positive (or negative, or neutral) if the restriction of $\rho$ to a fiber of $F$ is positive (or negative, or neutral). Similarly, suppose $\rho$ is a positive or negative monomials map, $I_{1}$ and $I_{2}$ are one-element sets, and $\mathcal{A}_{1}$ and $\mathcal{A}_{2}$ are integer-valued functions on

$$
I_{1} \times\left(I_{0} \sqcup J\right) \quad \text { and } \quad I_{2} \times\left(I_{0} \sqcup J\right),
$$

respectively. If $\rho_{1}$ and $\rho_{2}$ are monomials maps of degrees $\mathcal{A}_{1}$ and $\mathcal{A}_{2}$, respectively, we write $\rho_{1}>_{\rho} \rho_{2}$ if this relation holds for the restriction to a fiber; see Definition 2.2. Due to the remark following this definition, the notions of nondegenerate, positive, negative, and neutral depend only on $\mathcal{A}$; the partial ordering relation depends only on $\mathcal{A}, \mathcal{A}_{1}$, and $\mathcal{A}_{2}$.

If $F$ is any (normed) vector bundle and $\delta \in \mathbb{R}^{+}$, we define the function $\beta_{\delta}$ on $F$ by:

$$
\beta_{\delta}: F \longrightarrow[0,1] \subset \mathbb{R}, \quad \beta_{\delta}(v)=\beta_{\delta}(|v|) .
$$

If $\rho$ is a positive or negative monomials map between vector bundle and $t \in \mathbb{R}^{+}$, we denote by $\operatorname{deg} \rho$ the oriented degree of the map $t^{-1} \beta_{\delta} \rho$ given by Proposition 2.3B

The next two definitions characterize the topological spaces with which we work. Ms-orbifolds, as described by Definition [2.10] include spaces of stable maps. Examples of pseudovarieties, as described by Definition 2.11, that we encounter are subspaces of spaces of stable maps that consist of elements corresponding to curves with specified singularities.

Definition 2.10 A compact topological orbifold $\overline{\mathcal{M}}=\mathcal{M}_{n} \sqcup \bigsqcup_{k=0}^{n-1} \mathcal{M}_{k}$ is a
mostly smooth, or ms-orbifold of dimension $n$ if (1) $\mathcal{M} \equiv \mathcal{M}_{n}$ is an open subset of $\overline{\mathcal{M}}$, and $\overline{\mathcal{M}}_{k}-\mathcal{M}_{k} \subset \bigcup_{j<k} \mathcal{M}_{j}$ for all
$k=0, \ldots, n ;$

(2) $\mathcal{M}_{k}$ is a smooth oriented orbifold of dimension $2 k$ for all $k=0, \ldots, n$;

(3) for each $k=0, \ldots, n-1$, there exist a smooth complex vector orbi-bundle $\mathcal{F}_{k} \longrightarrow \mathcal{M}_{k}$ and an identification $\phi_{k}: U_{k} \longrightarrow V_{k}$ of neighborhoods of $\mathcal{M}_{k}$ in $\mathcal{F}_{k}$ and in $\overline{\mathcal{M}}$ such that $\phi_{k}: \phi_{k}^{-1}(\mathcal{M}) \longrightarrow V_{k} \cap \mathcal{M}$ is an orientation-preserving diffeomorphism. 
There is no firm consensus about the correct definition of the orbifold category. For our purposes, we put the following, rather strong, requirements on the objects involved in Definition 2.10. Each smooth orbifold $\mathcal{M}_{k}$ of Definition 2.10] is the quotient of a smooth manifold $\widetilde{\mathcal{M}}_{k}$ by a smooth action of a compact Lie group $G_{k}$. All points of $\widetilde{\mathcal{M}}_{k}$ have finite stabilizers, and the set of points with nontrivial stabilizers has codimension at least two in $\widetilde{\mathcal{M}}_{k}$. In other words, this set is a finite union of smooth manifolds of dimension at most $2 k-2+\operatorname{dim} G_{k}$. In addition, there exists a vector-bundle splitting

$$
T \widetilde{\mathcal{M}}_{k}=T^{v} \widetilde{\mathcal{M}}_{k} \oplus T^{h} \widetilde{\mathcal{M}}_{k}
$$

where $T^{v} \widetilde{\mathcal{M}}_{k}$ is the vertical tangent bundle and $T^{h} \widetilde{\mathcal{M}}_{k}$ is a complex vector bundle on which $G_{k}$ acts by complex-linear automorphisms. We call $\mathcal{F}_{k} \longrightarrow$ $\mathcal{M}_{k}$ a smooth complex vector orbi-bundle if there exists a smooth complex vector bundle $\widetilde{\mathcal{F}}_{k} \longrightarrow \widetilde{\mathcal{M}}_{k}$ on which $G_{k}$ acts smoothly.

By a compact topological orbifold $\overline{\mathcal{M}}$, we mean the quotient of a compact Hausdorff topological space

$$
\widetilde{\mathcal{M}}=\widetilde{\mathcal{M}}_{n} \sqcup \bigsqcup_{i=0}^{n-1} \widetilde{\mathcal{M}}_{k}^{\prime}
$$

by a continuous action of a compact Lie group $G$. For the purposes of Definition 2.10, $\widetilde{\mathcal{M}}_{k}^{\prime}$ denotes the preimage of $\mathcal{M}_{k}$ under the quotient projection map $\widetilde{\mathcal{M}} \longrightarrow \overline{\mathcal{M}}, G=G_{n}$, and the restriction of the continuous $G$-action to $\widetilde{\mathcal{M}}_{n}$ agrees with the smooth $G_{n}$-action of the previous paragraph. Condition (3) of Definition 2.10 means that there exist

(3a) a splitting $G_{k}=G \times G_{k}^{\prime}$;

(3b) a $G_{k}$-invariant neighborhood $\widetilde{U}_{k}$ of $\widetilde{\mathcal{M}}_{k}$ in $\widetilde{F}_{k}$;

(3c) a $G$-invariant neighborhood $\widetilde{V}_{k}$ of $\widetilde{\mathcal{M}}_{k}^{\prime}$ in $\widetilde{\mathcal{M}}$;

(3d) a $G$-equivariant topological $G_{k}^{\prime}$-fibration $\widetilde{\phi}_{k}: \widetilde{U}_{k} \longrightarrow \widetilde{V}_{k}$ such that

(3d-i) $\quad \widetilde{\phi}_{k}\left(\widetilde{\mathcal{M}}_{k}\right)=\widetilde{\mathcal{M}}_{k}^{\prime}$ and $\widetilde{\phi}_{k}: \widetilde{\phi}_{k}^{-1}\left(\widetilde{\mathcal{M}}_{n}\right) \longrightarrow \widetilde{V}_{k} \cap \widetilde{\mathcal{M}}_{n}$ is smooth;

(3d-ii) for each $x \in \widetilde{\phi}_{k}^{-1}\left(\widetilde{\mathcal{M}}_{n}\right)$, the map $\left.\pi_{\widetilde{\phi}_{k}(x)}^{h} \circ d \widetilde{\phi}_{k}\right|_{x}: T_{x}^{h} \widetilde{\mathcal{M}}_{k} \longrightarrow T_{\widetilde{\phi}_{k}(x)}^{h} \widetilde{\mathcal{M}}$ is an orientation-preserving isomorphism.

Throughout the rest of the paper by a vector bundle over a smooth orbifold we will mean a smooth complex normed vector orbi-bundle. With notation as above, this means that $\widetilde{\mathcal{F}}_{k}$ carries a $G_{k}$-invariant Hermitian inner-product. Similarly, $V \longrightarrow \overline{\mathcal{M}}$ is a vector bundle if 
(1) $V$ is the quotient of a topological Hermitian vector bundle $\widetilde{V} \longrightarrow \widetilde{\mathcal{M}}$ by an action of $G$;

(2) $V \mid \mathcal{M}_{k}$ is a vector bundle for all $i=0, \ldots, n$.

In (1), the action of $G$ preserves the Hermitian structure.

If both $\overline{\mathcal{M}}=\mathcal{M}_{n} \sqcup \bigsqcup_{k=0}^{n-1} \mathcal{M}_{k}$ and $\overline{\mathcal{M}}^{\prime}=\mathcal{M}_{n^{\prime}} \sqcup \bigsqcup_{k=0}^{n^{\prime}-1} \mathcal{M}_{k}^{\prime}$ are ms-orbifolds, a continuous map $\pi: \overline{\mathcal{M}} \longrightarrow \overline{\mathcal{M}}^{\prime}$ will called an ms-map if for each $k=0, \ldots, n$, there exists $k^{\prime}=0, \ldots, n^{\prime}$ such that $\pi: \mathcal{M}_{k} \longrightarrow \mathcal{M}_{k^{\prime}}^{\prime}$ is a smooth map.

Definition 2.11 Let $\overline{\mathcal{M}}$ be an ms-orbifold as in Definition 2.10.

(1) A smooth $2 m$-dimensional oriented suborbifold $\mathcal{S}$ of $\mathcal{M}$ is an $m$-pseudocycle in $\overline{\mathcal{M}}$ if $\overline{\mathcal{S}}-\mathcal{S}$ is contained in $\bigsqcup_{k=0}^{n-1} \mathcal{M}_{k}$ and $\overline{\mathcal{S}} \cap \mathcal{M}_{k}$ is contained in a finite union of smooth suborbifolds of $\mathcal{M}_{k}$ of dimension at most $2 m-2$.

(2) A pseudocycle $\mathcal{S}$ is a pseudovariety if $\mathcal{S}$ is a smooth submanifold of $\mathcal{M}$.

This definition of pseudocycle is a variation on that of [10, Chapter 7] and 14. Section 1]. For fairly straightforward topological reasons, every pseudocycle of [10] and [14] determines an integral homology class. For nearly the same reasons, every pseudocycle $\mathcal{S}$ of Definition 2.11 determines an element of $H_{2 m}(\overline{\mathcal{M}} ; \mathbb{Q})$. If $\alpha \in H^{2 m}(\overline{\mathcal{M}} ; \mathbb{Q})$, we denote the evaluation of $\alpha$ on this homology class by $\langle\alpha, \overline{\mathcal{S}}\rangle$. We write $\partial \overline{\mathcal{S}}$ for the boundary of $\mathcal{S}$, ie the set $\overline{\mathcal{S}}-\mathcal{S}$.

If $\mathcal{S}$ is a pseudovariety in $\overline{\mathcal{M}}$, by a vector bundle $V \longrightarrow \overline{\mathcal{S}}$ we will mean a topological Hermitian vector bundle over a neighborhood $U_{V}$ of $\overline{\mathcal{S}}$ in $\overline{\mathcal{M}}$ such that for each $k=0, \ldots, n$ the restriction of $V$ to $U_{V} \cap \mathcal{M}_{k}$ is a smooth Hermitian bundle. Similarly, we denote by $\Gamma(\overline{\mathcal{S}} ; V)$ the space of continuous sections of $V$ over $U_{V}$ that restrict to smooth sections on $U_{V} \cap \mathcal{M}_{k}$ for all $k=0, \ldots, n$. For the sake of simplicity, we restrict the presentation of our main topological tools to vector bundles over pseudovarieties as this is sufficient for the purposes of counting rational curves in projective spaces.

Throughout the paper, we assume that every smooth oriented manifold $\mathcal{Z}$ comes with a system of trivializations, ie a smooth map

$$
\vartheta_{\mathcal{Z}}: T \mathcal{Z}_{\delta} \longrightarrow \mathcal{Z}
$$

where $\delta \in C\left(\mathcal{Z} ; \mathbb{R}^{+}\right)$, such that $\vartheta_{\mathcal{Z}} \mid T_{b} \mathcal{Z}_{\delta(b)}$ is an orientation-preserving diffeomorphism onto an open neighborhood of $b$ in $\mathcal{Z}$ that sends $(b ; 0)$ to $b$. If 
$V \longrightarrow \mathcal{Z}$ is a vector bundle, we assume that such a map $\vartheta_{\mathcal{Z}}$ comes with a choice of a lift of $\vartheta_{\mathcal{Z}}$ to a bundle identification

$$
\vartheta_{\mathcal{Z} ; V}:\left.\pi_{T \mathcal{Z}}^{*} V\right|_{T \mathcal{Z}_{\delta}} \longrightarrow V
$$

ie an isomorphism of smooth Hermitian vector bundles that restricts to the identity over $\mathcal{Z} \subset T \mathcal{Z}$. If $V$ is given as a direct sum of proper subbundles $V_{i}$, $\vartheta_{\mathcal{Z} ; V}$ will be assumed to be induced by the identifications $\vartheta_{\mathcal{Z} ; V_{i}}$. If $\mathcal{M}$ is a smooth oriented manifold, $E \longrightarrow \mathcal{M}$ is a vector bundle, and $\kappa \in \Gamma(\mathcal{M} ; E)$ is a section transversal to the zero set, we assume that the smooth oriented submanifold $\mathcal{Z}=\kappa^{-1}(0)$ comes with a normal-neighborhood model, ie a smooth map

$$
\vartheta_{\kappa}: E_{\delta} \longrightarrow \mathcal{M}
$$

for some $\delta \in C\left(\mathcal{Z} ; \mathbb{R}^{+}\right)$, which is an orientation-preserving diffeomorphism onto an open neighborhood of $\mathcal{Z}$ in $\mathcal{M}$ such that $\vartheta_{\kappa} \mid \mathcal{Z}$ is the identity map. If $V \longrightarrow \mathcal{M}$ is a vector bundle, $\vartheta_{\kappa}$ comes with a choice of a bundle identification

$$
\vartheta_{\kappa ; V}:\left.\left.\pi_{E}^{*} V\right|_{E_{\delta}} \longrightarrow V\right|_{\operatorname{Im} \vartheta_{\kappa}}
$$

which respects vector-bundle splittings as above. Furthermore,

$$
\kappa\left(\vartheta_{\kappa}(b ; w)\right)=\vartheta_{\kappa ; E}(b ; w)
$$

for all $(b ; w) \in E_{\delta}$. We will often only imply these identifications in equations involving vector-bundle sections.

Definition 2.12 Suppose $\overline{\mathcal{M}}$ is an ms-manifold as in Definition 2.10, $\mathcal{S}$ is a pseudovariety in $\overline{\mathcal{M}}$ as in part (2) of Definition 2.11 and $\mathcal{Z}$ is a smooth submanifold of $\mathcal{M}_{k}$ for some $k=0, \ldots, n$.

(1) A regularization of $\mathcal{Z}$ in $\overline{\mathcal{M}}$ is a tuple $\left(U_{\mathcal{Z}}, E, \kappa,\left(\mathcal{F}_{k ; j}\right)_{j \in J(\mathcal{Z})}\right)$, where

(1a) $U_{\mathcal{Z}}$ is a neighborhood of $\mathcal{Z}$ in $\mathcal{M}_{k}, E \longrightarrow U_{\mathcal{Z}}$ is a vector bundle, and $\kappa \in \Gamma\left(U_{\mathcal{Z}} ; V\right)$ is a section transversal to the zero set such that $\mathcal{Z}=\kappa^{-1}(0)$

(1b) $\mathcal{F}_{k ; j} \longrightarrow \mathcal{U}_{\mathcal{Z}}$ is a non-zero subbundle of $\mathcal{F}_{k} \mid U_{\mathcal{Z}}$ for each $j \in J(\mathcal{Z})$ such that $\mathcal{F}_{k} \mid U_{\mathcal{Z}}=\bigoplus_{j \in J(\mathcal{Z})} \mathcal{F}_{k ; j}, \phi_{k}\left(U_{k} \cap Y\left(\mathcal{F}_{k} \mid U_{\mathcal{Z}} ; J(\mathcal{Z})\right)\right) \subset \partial \overline{\mathcal{M}}$, and $\phi_{k}\left(\left(U_{k} \mid U_{\mathcal{Z}}\right)-Y\left(\mathcal{F}_{k} \mid U_{\mathcal{Z}} ; J(\mathcal{Z})\right)\right) \subset \mathcal{M}$.

(2) A model for $\mathcal{Z}$ in $\mathcal{S}$ is a tuple $\left(U_{\mathcal{Z}}, E, \kappa,\left(\mathcal{F}_{k ; j}\right)_{j \in J(\mathcal{Z})} ; \mathcal{O}_{\mathcal{Z}}, \psi_{\mathcal{Z}}\right)$, where

(2a) $\left(U_{\mathcal{Z}}, E, \kappa,\left(\mathcal{F}_{k ; j}\right)_{j \in J(\mathcal{Z})}\right)$ is a regularization of $\mathcal{Z}$ in $\overline{\mathcal{M}}$;

(2b) $\mathcal{O}_{\mathcal{Z}} \longrightarrow U_{\mathcal{Z}}$ is a vector bundle of $\operatorname{rank} \frac{1}{2}(\operatorname{dim} \mathcal{M}-\operatorname{dim} \mathcal{S})$;

(2c) $\psi_{\mathcal{Z}}: \mathcal{F}_{k} \longrightarrow \mathcal{O}_{\mathcal{Z}}$ is a bundle map such that 
(2c-i) $\psi_{\mathcal{Z}}$ is smooth outside of $Y\left(\mathcal{F}_{k} ; J(\mathcal{Z})\right)$;

(2c-ii) if $v \in U_{k} \mid U_{\mathcal{Z}}, \phi_{k}(v) \in \mathcal{S}$ if and only if $\psi_{\mathcal{Z}}(v)=0$.

Suppose $V \longrightarrow \overline{\mathcal{S}}$ is a vector bundle and $\left(U_{\mathcal{Z}}, E, \kappa,\left(\mathcal{F}_{k ; j}\right)_{j \in J(\mathcal{Z})}\right)$ is a regularization of $\mathcal{Z}$ in $\overline{\mathcal{M}}$. In such a case, we assume that the tuple

$$
\left(U_{\mathcal{Z}}, E, \kappa,\left(\mathcal{F}_{k ; j}\right)_{j \in J(\mathcal{Z})}\right)
$$

implicitly encodes a Hermitian vector-bundle isomorphism

$$
\vartheta_{U_{\mathcal{Z}} ; V}:\left.\left.\pi_{\mathcal{F}_{k}}^{*} V\right|_{\phi_{k}^{-1}\left(U_{V}\right) \mid\left(U_{V} \cap U_{\mathcal{Z}}\right)} \longrightarrow V\right|_{U_{V} \cap \phi_{k}\left(U_{k} \mid\left(U_{V} \cap U_{\mathcal{Z}}\right)\right)}
$$

that covers the map $\phi_{k}$ and restricts to the identity over $U_{V} \cap U_{\mathcal{Z}}$. This isomorphism is to be smooth over the complement of $Y\left(\mathcal{F}_{k} \mid U_{\mathcal{Z}} ; J(\mathcal{Z})\right)$. Along with the map $\vartheta_{k ; V}$, we then obtain an identification

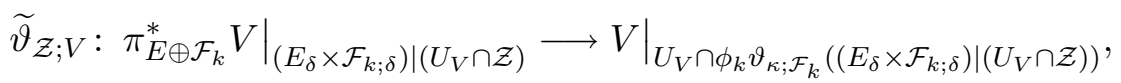

covering the map $\phi_{k} \circ \vartheta_{\kappa ; \mathcal{F}_{k}}$ for $\delta \in C\left(\mathcal{Z} \cap U_{V} ; \mathbb{R}^{+}\right)$sufficiently small.

Definition 2.13 Suppose $\mathcal{M}$ is a smooth manifold, $F, V \longrightarrow \mathcal{M}$ are vector bundles, and $\Omega$ is an open subset of $F$.

(1) A smooth bundle map $\varepsilon: \Omega \longrightarrow V$ is $C^{0}{ }_{-n}$ egligible if $\lim _{v \longrightarrow 0} \varepsilon(v)=0$.

(2) A $C^{0}$-negligible map $\varepsilon: \Omega \longrightarrow V$ is $C^{1}{ }^{-}$negligible if $\lim _{v \longrightarrow 0} D_{\mathcal{M}} \varepsilon(v)=0$, where $D_{\mathcal{M}} \varepsilon$ denotes the differentiation of $\varepsilon$ along $\mathcal{M}$ with respect to some connections in $F$ and $V$.

(3) If $\rho$ is a positive or negative monomials map on $F=\bigoplus_{i \in I} F_{i}$ and $\widetilde{\rho}$ is a monomials map on $F$ with values in a line bundle $L$, a smooth bundle map $\varepsilon: \Omega \longrightarrow V$ is $(\rho, \widetilde{\rho})$-controlled if there exist monomials maps $\rho_{1}, \ldots, \rho_{N}$ on $F$ with values in $L$ such that

$$
\widetilde{\rho}>_{\rho} \rho_{j} \quad \text { for all } j=1, \ldots, N, \quad \text { and } \lim _{\substack{v \in \Omega-Y(F ; I) \\ v \longrightarrow 0}}\left(\sum_{j=1}^{N}\left|\rho_{j}(v)\right|\right)^{-1}|\varepsilon(v)|<\infty \text {. }
$$

Definition 2.14 Suppose $\overline{\mathcal{M}}, \mathcal{S}$, and $\mathcal{Z} \subset \mathcal{M}_{k}$ are as in Definition 2.12 $V \longrightarrow \overline{\mathcal{S}}$ is a vector bundle, and $s \in \Gamma(\mathcal{S} ; V)$.

(1) A semi-regularization of $s$ near $\mathcal{Z}$ is a tuple

$$
\left(U_{\mathcal{Z}}, E, \kappa,\left(\mathcal{F}_{k ; j}\right)_{j \in J(\mathcal{Z})} ; \mathcal{O}_{\mathcal{Z}}^{-} \oplus \mathcal{O}_{\mathcal{Z}}^{+}, \psi_{\mathcal{Z}}^{-} \oplus \psi_{\mathcal{Z}}^{+} ; \widetilde{\mathcal{F}}, \rho, \alpha_{+}, \alpha_{V}, \nu^{*}\right)
$$

where 
(1a) $\left(U_{\mathcal{Z}}, E, \kappa,\left(\mathcal{F}_{k ; j}\right)_{j \in J(\mathcal{Z})} ; \mathcal{O}_{\mathcal{Z}}^{-} \oplus \mathcal{O}_{\mathcal{Z}}^{+}, \psi_{\mathcal{Z}}^{-} \oplus \psi_{\mathcal{Z}}^{+}\right)$is model for $\mathcal{Z}$ in $\mathcal{S}$ such that $\operatorname{rk} E \geq \operatorname{rk} \mathcal{O}_{\mathcal{Z}}^{-}$

(1b) $\tilde{\mathcal{F}}=\bigoplus_{i \in \widetilde{I}(\mathcal{Z})} \widetilde{\mathcal{F}}_{i} \longrightarrow U_{\mathcal{Z}}$ is a vector bundle and

$$
\rho: \mathcal{F}_{k} \mid \mathcal{U}_{\mathcal{Z}}-Y\left(\mathcal{F}_{k} \mid \mathcal{U}_{\mathcal{Z}} ; J(\mathcal{Z})\right) \longrightarrow \widetilde{\mathcal{F}}
$$

is a smooth bundle map;

(1c) $\quad \nu^{*} \in \Gamma\left(U_{\mathcal{Z}} ; \mathcal{O}_{\mathcal{Z}}^{+} \oplus V\right)$ and $\alpha_{+} \oplus \alpha_{V} \in \Gamma\left(U_{\mathcal{Z}} ; \operatorname{Hom}\left(\widetilde{\mathcal{F}} ; \mathcal{O}_{\mathcal{Z}}^{+} \oplus V\right)\right)$ is an injective linear map such that $\alpha_{+}$is onto along $\mathcal{Z}$;

(1d) there exist $\delta \in C^{\infty}\left(\mathcal{Z} \cap U_{V} ; \mathbb{R}^{+}\right)$and a $C^{0}$-negligible map

$$
\widetilde{\varepsilon}_{+, V}: \mathcal{F}_{k} \mid \mathcal{U}_{\mathcal{Z}}-Y\left(\mathcal{F}_{k} \mid \mathcal{U}_{\mathcal{Z}} ; J(\mathcal{Z})\right) \longrightarrow \operatorname{Hom}\left(\widetilde{\mathcal{F}} ; \mathcal{O}_{\mathcal{Z}}^{+} \oplus V\right)
$$

such that

$$
\left(\psi_{+}(b ; v), s(b ; v)\right)=\left(\alpha_{+} \rho(b ; v), \alpha_{V} \rho(b ; v)\right)+\nu^{*}(b)+\widetilde{\varepsilon}_{+, V}(b ; v) \rho(b ; v)
$$

for all $(b ; v) \in \mathcal{F}_{k ; \delta}$ such that $\phi_{k}(b ; v) \in \mathcal{S}$.

(2) A semi-regularization

$$
\left(U_{\mathcal{Z}}, E, \kappa,\left(\mathcal{F}_{k ; j}\right)_{j \in J(\mathcal{Z})} ; \mathcal{O}_{\mathcal{Z}}^{-} \oplus \mathcal{O}_{\mathcal{Z}}^{+}, \psi_{\mathcal{Z}}^{-} \oplus \psi_{\mathcal{Z}}^{+} ; \widetilde{\mathcal{F}}, \rho, \alpha_{+}, \alpha_{V}, \nu^{*}\right)
$$

is hollow if $\operatorname{rk} \widetilde{\mathcal{F}}_{k}<\operatorname{rk} \mathcal{F}_{k}$ and either $\nu^{*}=0$ or the bundle map

$$
\widetilde{\mathcal{F}} \longrightarrow \mathcal{O}^{+} \oplus V, \quad(b ; \widetilde{v}) \longrightarrow\left\{\alpha_{+} \oplus \alpha_{V}\right\}(b ; \widetilde{v})+\nu^{*}(b),
$$

does not vanish over $\mathcal{Z}$.

(3) A semi-regularization

$$
\left(U_{\mathcal{Z}}, E, \kappa,\left(\mathcal{F}_{k ; j}\right)_{j \in J(\mathcal{Z})} ; \mathcal{O}_{\mathcal{Z}}^{-} \oplus \mathcal{O}_{\mathcal{Z}}^{+}, \psi_{\mathcal{Z}}^{-} \oplus \psi_{\mathcal{Z}}^{+} ; \widetilde{\mathcal{F}}, \rho, \alpha_{+}, \alpha_{V}, \nu^{*}\right)
$$

is neutral if $\rho$ is a neutral monomials map, $\alpha_{+} \mid Y(\widetilde{\mathcal{F}} ;\{i\})$ is onto over $\mathcal{Z}$ for all $i \in \widetilde{I}(\mathcal{Z})$, and $\nu^{*}=0$.

Definition 2.15 Suppose $\overline{\mathcal{M}}, \mathcal{S}, \mathcal{Z} \subset \mathcal{M}_{k}, V \longrightarrow \overline{\mathcal{S}}$, and $s \in \Gamma(\mathcal{S} ; V)$ are as in Definition 2.14. A regularization of $s$ near $\mathcal{Z}$ is a tuple

$$
\begin{aligned}
&\left(U_{\mathcal{Z}},\left(E_{i}\right)_{i \in I(\mathcal{Z})},\left(\kappa_{i}\right)_{i \in I(\mathcal{Z})},\left(\mathcal{F}_{k ; j}\right)_{j \in J(\mathcal{Z})}\right. \\
&\left.\mathcal{O}_{\mathcal{Z}}^{-} \oplus \mathcal{O}_{\mathcal{Z}}^{+}, \psi_{\mathcal{Z}}^{-} \oplus \psi_{\mathcal{Z}}^{+} ; \widetilde{\mathcal{F}}, \rho, \alpha_{+}, \alpha_{V} ;\left(\widetilde{E}_{i}\right)_{i \in I(\mathcal{Z})}, \widetilde{\rho}, \alpha_{-}\right),
\end{aligned}
$$

where

(1) $\quad E_{i} \longrightarrow U_{\mathcal{Z}}$ is a vector bundle for each $i \in I(\mathcal{Z})$; 
(2) with $E=\bigoplus_{i \in I(\mathcal{Z})} E_{i}$ and $\kappa=\left(\kappa_{i}\right)_{i \in I(\mathcal{Z})}$,

$$
\left(U_{\mathcal{Z}}, E, \kappa,\left(\mathcal{F}_{k ; j}\right)_{j \in J(\mathcal{Z})} ; \mathcal{O}_{\mathcal{Z}}^{-} \oplus \mathcal{O}_{\mathcal{Z}}^{+}, \psi_{\mathcal{Z}}^{-} \oplus \psi_{\mathcal{Z}}^{+} ; \widetilde{\mathcal{F}}, \rho, \alpha_{+}, \alpha_{V}, 0\right)
$$

is a semi-regularization of $s$ near $\mathcal{Z}$ such that $\rho$ is a positive or negative monomials map and $\alpha_{+} \mid Y(\widetilde{\mathcal{F}} ;\{i\})$ is onto over $\mathcal{Z}$ for all $i \in \widetilde{I}(\mathcal{Z})$;

(3) $\tilde{\rho}=\left(\widetilde{\rho}_{i}\right)_{i \in I(\mathcal{Z})}$ is a degree- $\mathcal{A}$ monomials map on $E \oplus \mathcal{F}_{k}$ with values in $\widetilde{E} \equiv \bigoplus_{i \in I(\mathcal{Z})} \widetilde{E}_{i}$, for some integer-valued function $\mathcal{A}$ on $I(\mathcal{Z}) \times J(\mathcal{Z})$;

(4) $\alpha_{-} \in \Gamma\left(\mathcal{Z} ; \operatorname{Hom}\left(\widetilde{E}, \mathcal{O}_{\mathcal{Z}}^{-}\right)\right)$is an isomorphism on every fiber;

(5) there exist $\delta \in C\left(\mathcal{Z} \cap U_{V} ; \mathbb{R}^{+}\right)$and for each $i \in I(\mathcal{Z})$ a $C^{1}$-negligible map and a $\left(\rho, \widetilde{\rho}_{i}\right)$-controlled map,

$$
\begin{gathered}
\widetilde{\varepsilon}_{i}^{-}: \mathcal{F}_{k} \mid \mathcal{U}_{\mathcal{Z}}-Y\left(\mathcal{F}_{k} \mid \mathcal{U}_{\mathcal{Z}} ; J(\mathcal{Z})\right) \longrightarrow \operatorname{Hom}\left(\widetilde{F}_{i}(\widetilde{\rho}), \alpha_{i}\left(\widetilde{E}_{i}\right)\right) \quad \text { and } \\
\varepsilon_{i}^{-}: \mathcal{F}_{k} \mid \mathcal{U}_{\mathcal{Z}}-Y\left(\mathcal{F}_{k} \mid \mathcal{U}_{\mathcal{Z}} ; J(\mathcal{Z})\right) \longrightarrow \alpha_{i}\left(\widetilde{E}_{i}\right)
\end{gathered}
$$

such that

$$
\psi_{i}^{-}(b ; v)=\alpha_{-} \widetilde{\rho}_{i}\left(b ; \kappa_{i}(b), v\right)+\widetilde{\varepsilon}_{i}^{-}(b ; v) \widetilde{\rho}_{i}(v)+\varepsilon_{i}^{-}(b ; v) \quad \text { for all }(b ; v) \in \mathcal{F}_{k ; \delta},
$$

where $\psi_{i}^{-}$denotes the $i$ th component of $\psi^{-}$under the decomposition $\mathcal{O}_{\mathcal{Z}}^{-} \mid \mathcal{Z}=$ $\bigoplus_{i \in I(\mathcal{Z})} \alpha_{-}\left(\widetilde{E}_{i}\right)$.

Remark Proposition 3.5 ensures that $\psi^{-}$admits an expansion as in (5) of Definition 2.15] in most cases one would encounter in counting rational curves in projective spaces. However, this expansion is not needed if $\mathcal{Z}$ is $s$-hollow or $s$-neutral; see Definition 2.17 and Proposition 2.18B.

If $\mathcal{F}, E \longrightarrow \mathcal{Z}$ are vector bundles and $\alpha \in \Gamma(\mathcal{Z} ; \operatorname{Hom}(\mathcal{F}, E))$, let

$$
\widetilde{\alpha} \in \Gamma\left(\mathbb{P} \mathcal{F} ; \operatorname{Hom}\left(\gamma_{\mathcal{F}}, \pi_{\mathbb{P} \mathcal{F}}^{*} E\right)\right)
$$

denote the section induced by $\alpha$. Here $\gamma_{\mathcal{F}} \longrightarrow \mathbb{P F}$ is the tautological line bundle, while $\pi_{\mathbb{P} \mathcal{F}}: \mathbb{P} \mathcal{F} \longrightarrow \mathcal{Z}$ is the bundle projection map.

If $\mathcal{F}, E \longrightarrow \mathcal{Z}$ and $\alpha$ are as above, a closure of $(\mathcal{Z}, \alpha)$ is a tuple $\left(\overline{\mathcal{M}}^{\prime}, \mathcal{F}^{\prime}, E^{\prime}\right)$, where $\overline{\mathcal{M}}^{\prime}$ is an ms-manifold containing $\mathcal{Z}$ as a pseudovariety, and $\mathcal{F}^{\prime}$ and $E^{\prime}$ are vector bundles over $\overline{\mathcal{Z}}$ that restrict to $\mathcal{F}$ and $E$, respectively, over $\mathcal{Z}$.

Definition 2.16 Let $\overline{\mathcal{M}}, \mathcal{S}, \mathcal{Z}, V$, and $s$ be as in Definition 2.14, A regularization

$$
\begin{aligned}
&\left(U_{\mathcal{Z}},\left(E_{i}\right)_{i \in I(\mathcal{Z})},\left(\kappa_{i}\right)_{i \in I(\mathcal{Z})},\left(\mathcal{F}_{k ; j}\right)_{j \in J(\mathcal{Z})}\right. \\
&\left.\mathcal{O}_{\mathcal{Z}}^{-} \oplus \mathcal{O}_{\mathcal{Z}}^{+}, \psi_{\mathcal{Z}}^{-} \oplus \psi_{\mathcal{Z}}^{+} ; \widetilde{\mathcal{F}}, \rho, \alpha_{+}, \alpha_{V} ;\left(\widetilde{E}_{i}\right)_{i \in I(\mathcal{Z})}, \widetilde{\rho}, \alpha_{-}\right)
\end{aligned}
$$


of $s$ at $\mathcal{Z}$ is closable if $\left(\mathcal{Z}, \alpha_{V}\right)$ admits a closure $\left(\overline{\mathcal{M}}^{\prime}, \widetilde{\mathcal{F}}^{\prime}, \mathcal{O}^{\prime}\right)$ and $\mathbb{P} \widetilde{\mathcal{F}}^{\prime}$ admits an ms-orbifold structure such that

(1) $\overline{\mathcal{M}}^{\prime} \subset \overline{\mathcal{M}}, \pi_{\mathbb{P} \tilde{\mathcal{F}}^{\prime}}: \mathbb{P} \widetilde{\mathcal{F}}^{\prime} \longrightarrow \overline{\mathcal{M}}$ is an ms-map, and $\widetilde{\alpha}_{+}^{-1}(0)$ is a pseudovariety in $\mathbb{P} \widetilde{\mathcal{F}}^{\prime}$;

(2) $\partial \overline{\widetilde{\alpha}_{+}^{-1}(0)}$ is a union of subsets $\mathcal{Z}_{i}$ such that $\widetilde{\alpha}_{V}$ admits a semi-regularization at each $\mathcal{Z}_{i}$.

Definition 2.17 Let $\overline{\mathcal{M}}, \mathcal{S}, V$, and $s$ be as in Definition 2.14]

(1) $\mathcal{Z} \subset \mathcal{M}_{k}$ is $s$-hollow (neutral, regular) if $s$ admits a hollow semi-regularization (neutral semi-regularization, closable regularization) near $\mathcal{Z}$.

(2) A section $s$ is regular if $s$ is transversal to the zero set and there exists a finite collection $\left\{\mathcal{Z}_{i}\right\}_{i \in I_{s}}$ of smooth disjoint manifolds in $\overline{\mathcal{M}}$ such that

(2a) $\partial \overline{\mathcal{S}} \subset \bigsqcup_{i \in I_{s}} \mathcal{Z}_{i}$ and $\overline{\mathcal{Z}}_{i}-\mathcal{Z}_{i} \subset \bigcup_{\operatorname{dim} \mathcal{Z}_{j}<\operatorname{dim} \mathcal{Z}_{i}} \mathcal{Z}_{j}$ for all $i \in I_{s}$;

(2b) $\mathcal{Z}_{i}$ is either $s$-hollow, $s$-neutral, or $s$-regular for every $i \in I_{s}$.

Suppose $s \in \Gamma(\mathcal{S} ; V)$ is a regular section as in (2) of Definition 2.17. Let $\left\{\mathcal{Z}_{i}\right\}_{i \in I_{s}^{*}}$ be the subcollection of $s$-regular subsets. To each $i \in I_{s}^{*}$, we associate the tuple

$$
\varrho_{i}=\left(\overline{\mathcal{M}}_{i}, \mathcal{S}_{i}, \gamma_{i}, \mathcal{O}_{i}, \alpha_{i} ; \operatorname{deg} \rho_{i}\right),
$$

where, with notation as in Definitions 2.15] and 2.16,

$$
\begin{gathered}
\overline{\mathcal{M}}_{i}=\mathbb{P} \widetilde{\mathcal{F}}^{\prime}, \quad \mathcal{S}_{i}=\widetilde{\alpha}_{+}^{-1}(0) \subset \overline{\mathcal{M}}_{i}, \quad \gamma_{i}=\gamma_{\widetilde{\mathcal{F}}^{\prime}} \longrightarrow \overline{\mathcal{S}}_{i}, \quad \mathcal{O}_{i}=\pi_{\widetilde{\mathcal{F}}^{\prime}}^{*} V \longrightarrow \overline{\mathcal{S}}_{i}, \\
\alpha_{i}=\widetilde{\alpha}_{V} \in \Gamma\left(\mathcal{S}_{i}, \operatorname{Hom}\left(\gamma_{i}, \mathcal{O}_{i}\right)\right),
\end{gathered}
$$

are the objects corresponding to $\mathcal{Z}_{i}$. We will write $\operatorname{deg} \varrho_{i}$ for $\operatorname{deg} \rho_{i}$.

\subsection{Contributions to the Euler class}

In this subsection, we describe the topological part of the computational method of this paper:

Proposition 2.18A Suppose $\mathcal{S}$ is an $m$-pseudovariety in an ms-orbifold $\overline{\mathcal{M}}$ and $E, \mathcal{O} \longrightarrow \overline{\mathcal{S}}$ are vector bundles such that $\mathrm{rk} E=1$ and $\mathrm{rk} \mathcal{O}=m+1$. If $\alpha \in \Gamma(\mathcal{S} ; \operatorname{Hom}(E, \mathcal{O}))$ is a regular section, for a dense open subset $\Gamma_{\alpha}(\overline{\mathcal{S}} ; \mathcal{O})$ of sections $\bar{\nu} \in \Gamma(\overline{\mathcal{S}} ; \mathcal{O})$, the affine map

$$
\psi_{\alpha, \bar{\nu}} \equiv \alpha+\bar{\nu}: E \mid \mathcal{S} \longrightarrow \mathcal{O}, \quad \psi_{\alpha, \bar{\nu}}(b ; v)=\alpha_{b}(v)+\bar{\nu}_{b},
$$


is transversal to the zero set. The set $\psi_{\alpha, \bar{\nu}}^{-1}(0)$ is finite, and its signed cardinality ${ }^{ \pm}\left|\psi_{\alpha, \bar{\nu}}^{-1}(0)\right|$ is dependent only on $\alpha$ and is given by

$$
N(\alpha) \equiv^{ \pm}\left|\psi_{\alpha, \bar{\nu}}^{-1}(0)\right|=\left|\alpha^{\perp-1}(0)\right|,
$$

where $\alpha^{\perp} \in \Gamma(\mathcal{S} ; \operatorname{Hom}(E, \mathcal{O} / \mathbb{C}))$ is the composition of $\alpha$ with projection map onto the quotient of $\mathcal{O}$ by a generic trivial line subbundle.

Proposition 2.18B Suppose $\mathcal{S}$ is an $m$-pseudovariety in ms-orbifold $\overline{\mathcal{M}}$, $V \longrightarrow \overline{\mathcal{S}}$ is vector bundle of rank $m$, such that $e(V)$ is the restriction of a cohomology class on $\overline{\mathcal{M}}$, and $s \in \Gamma(S ; V)$ is a regular section. Then, $s^{-1}(0)$ is a finite set, and

$$
{ }^{ \pm}\left|s^{-1}(0)\right|=\langle e(V), \overline{\mathcal{S}}\rangle-\sum_{i \in I_{s}^{*}} \operatorname{deg} \varrho_{i} \cdot N\left(\varrho_{i}\right) \equiv\langle e(V), \overline{\mathcal{S}}\rangle-\mathcal{C}_{\partial \overline{\mathcal{S}}}(s),
$$

where $I_{s}^{*}$ is a complete collection of effective regularizations of $s$ on $\overline{\mathcal{S}}$, as in the last paragraph of Subsection 2.2 and $N\left(\varrho_{i}\right) \equiv N\left(\alpha_{i}\right)$.

Remarks (1) Together the two propositions give a reductive procedure for counting the number of zeros of a section over the main stratum of a pseudovariety, provided that the section is "reasonably nice." Indeed, one application of both propositions reduces the rank of the target bundle by one. In the holomorphic category, every section is in fact "reasonably nice." By Proposition 3.5. many sections of interest to us also have the needed properties.

(2) In Proposition 2.18A $E$ can be a vector bundle of arbitrary rank, provided the rank of $\mathcal{O}$ is adjusted appropriately and the section $\widetilde{\alpha}$ is regular. In such a case, $N(\alpha)=N(\widetilde{\alpha})$. In fact, one can obtain such a reduction even if the original map $\alpha$ is a polynomial; see Subsection 3.3 in [17] for details. In addition, it is not necessary to assume that $\alpha$ does not vanish over $\mathcal{S}$. However, in practical applications, the boundary of $\overline{\mathcal{M}}$ can be enlarged to absorb the zero set of $\alpha$.

(3) If $E, E^{\prime} \longrightarrow \overline{\mathcal{M}}$ are vector bundles, $\rho \in \Gamma\left(\mathcal{S} ; \operatorname{Hom}\left(E, E^{\prime}\right)\right)$ is an isomorphism on every fiber, and $\alpha \in \Gamma(\mathcal{S} ; \operatorname{Hom}(E, \mathcal{O}))$, then $N(\alpha)=N\left(\alpha \circ \rho^{-1}\right)$, provided both numbers are defined. Note that the isomorphism $\rho$ is assumed to be defined over $\mathcal{S}$, and not over $\overline{\mathcal{S}}$. We will call the replacement of $\alpha$ by $\alpha \circ \rho^{-1}$ a rescaling of the linear map. A good choice of the isomorphism $\rho$ may greatly simplify the computation of the number $N(\alpha)$ via Propositions 2.18A and 2.18B In actual applications, our isomorphisms $\rho$ will be such that $N(\alpha)$ is defined if and only if $N\left(\alpha \circ \rho^{-1}\right)$ is defined.

(4) If $\alpha_{+}$and $\alpha_{V}$ are as in the last paragraph of Subsection 2.2

$$
N\left(\widetilde{\alpha}_{V}\right)=N\left(\alpha_{+} \oplus \alpha_{V}\right) .
$$


In particular, if $\mathcal{Z}_{i}$ is a finite set and thus $\alpha_{+} \oplus \alpha_{V}$ is an isomorphism over every point of $\mathcal{Z}_{i}$, then $N\left(\widetilde{\alpha}_{V}\right)=^{ \pm}\left|\mathcal{Z}_{i}\right|$.

For computational purposes, it is useful to observe that if $E \longrightarrow \overline{\mathcal{S}}$ is a vector bundle of rank $n$ such that $c(E)$ is the restriction of an element of $H^{*}(\overline{\mathcal{M}})$,

$$
\begin{aligned}
& \lambda_{E}^{n}+\sum_{k=1}^{k=n} c_{k}(E) \lambda_{E}^{n-k}=0 \in H^{2 n}(\mathbb{P} E ; \mathbb{Q}) \quad \text { and } \\
& \left\langle\mu \lambda_{E}^{n-1}, \mathbb{P} E \mid \overline{\mathcal{S}}\right\rangle=\langle\mu, \overline{\mathcal{S}}\rangle \quad \text { for all } \mu \in H^{2 m}(\overline{\mathcal{M}} ; \mathbb{Q}) ;
\end{aligned}
$$

the same formula in a more standard setting can be found in [1, Section 20], for example. In the situation of Proposition 2.18A but with $E$ of an arbitrary rank, Propositions 2.18A and 2.18B and equation (2.16) give

$$
N(\alpha)=\left\langle c(\mathcal{O}) c(E)^{-1}, \overline{\mathcal{S}}\right\rangle-\mathcal{C}_{\mathbb{P} E \mid \partial \overline{\mathcal{S}}}\left(\widetilde{\alpha}^{\perp}\right) .
$$

The last term above is zero if $\alpha$ extends to a section of $\operatorname{Hom}(E, \mathcal{O})$ over $\overline{\mathcal{S}}$ that has full rank over every point of $\overline{\mathcal{S}}$. In the computational sections of this papers, we view formulas (2.16) and (2.17) as parts of Propositions 2.18A and 2.18B

In the rest of this subsection, we prove Propositions 2.18A and 2.18B Before proceeding, we first comment on the topology on $\Gamma(\overline{\mathcal{S}} ; \mathcal{O})$ to which the first proposition makes an implicit reference. There are many topologies in which the statement of the proposition is valid. One of them is defined via convergence of sequences on compact subsets in the $C^{0}$-norm on $U_{\mathcal{O}}$ and the $C^{2}$-norm on compact subset of $U_{\mathcal{O}} \cap \mathcal{M}_{k}$; see [17, Subsection 3.2] for more details.

The proof of Proposition 2.18A is essentially the same as the proof of [17, Lemma 3.14]. The finiteness claim is proved as follows. Suppose $\left(b_{r}, v_{r}\right) \in E \mid \mathcal{S}$ is a sequence such that $\psi_{\alpha, \bar{\nu}}\left(b_{r}, v_{r}\right)=0$ and $\left\{b_{r}\right\}$ converges to some $b^{*} \in \partial \overline{\mathcal{S}}$. Let

$$
\left(U_{\mathcal{Z}}, E^{\prime}, \kappa,\left(\mathcal{F}_{k ; j}\right)_{j \in J(\mathcal{Z})} ; \mathcal{O}_{\mathcal{Z}}^{-} \oplus \mathcal{O}_{\mathcal{Z}}^{+}, \psi_{\mathcal{Z}}^{-} \oplus \psi_{\mathcal{Z}}^{+} ; \widetilde{\mathcal{F}}, \rho, \alpha_{+}, \alpha_{E^{*} \otimes \mathcal{O}}, \nu^{*}\right)
$$

be a semi-regularization of $\alpha$ at a submanifold $\mathcal{Z}$ of $\mathcal{M}_{k}$ containing $b^{*}$, as provided by (2) of Definition 2.17. By replacing $\mathcal{Z}$ with $\mathcal{Z} \cap U_{E} \cap U_{\mathcal{O}}$, if necessary, it can be assumed that $\mathcal{Z}_{i} \subset U_{E} \cap U_{\mathcal{O}}$. As in the proof of Lemma 3.12 in [17], from the sequence $\left\{\left(b_{r}, v_{r}\right)\right\}$ we can obtain a zero of the map

$$
\psi_{\alpha, \bar{\nu} ; \mathcal{Z}}: E \otimes \widetilde{\mathcal{F}} \longrightarrow E \otimes \mathcal{O}^{+} \oplus \mathcal{O}, \psi_{\alpha, \bar{\nu} ; \mathcal{Z}}(b ; v)=\left(\alpha_{+}(v), \alpha_{E^{*} \otimes \mathcal{O}}(v)+\bar{\nu}\right),
$$

if $\nu^{*}=0$. This is a bundle map over $\mathcal{Z}$. By (1c) of Definition 2.14, the first-component map is surjective. Thus, for a dense open subset of elements of $\Gamma(\overline{\mathcal{S}} ; \mathcal{O})$, the map $\psi_{\alpha, \bar{\nu} ; \mathcal{Z}}$ is transversal to the zero set. Assumptions on the 
dimension and the ranks imply that $\psi_{\alpha, \bar{\nu} ; \mathcal{Z}}(b ; v)$ does not vanish if $\bar{\nu}$ lies in this open dense subset; see (2) of Definition 2.12 and (1a)-(1c) of Definition 2.14. On the other hand, if $\bar{\nu}^{*} \neq 0$, we can obtain a zero of the map

$$
\begin{gathered}
\psi_{\alpha, \bar{\nu} ; \mathcal{Z}}: E \otimes \widetilde{\mathcal{F}} \oplus E \longrightarrow E \otimes \mathcal{O}^{+} \oplus \mathcal{O}, \\
\psi_{\alpha, \bar{\nu} ; \mathcal{Z}}(b ; w, v)=\left(\alpha_{+}(v), \alpha_{E^{*} \otimes \mathcal{O}}(v)+\bar{\nu}\right)+\nu^{*} w .
\end{gathered}
$$

In this case, rk $\widetilde{\mathcal{F}}<\operatorname{rk} \mathcal{F}_{k}$ and thus the map $\psi_{\alpha, \bar{\nu} ; \mathcal{Z}}$ again has no zeros if $\bar{\nu}$ is generic. We conclude that $\psi_{\alpha, \bar{\nu}}^{-1}(0)$ is a finite set. The independence of ${ }^{ \pm}\left|\psi_{\alpha, \bar{\nu}}^{-1}(0)\right|$ of the choice of $\bar{\nu}$ is shown by constructing a cobordism between $\psi_{\alpha, \bar{\nu}_{1}}^{-1}(0)$ and $\psi_{\alpha, \bar{\nu}_{2}}^{-1}(0)$; see part (5) of the proof of Lemma 2.22 below for a similar argument. The final claim of Proposition $2.18 \mathrm{~A}$ is nearly immediate from the definition of $\psi_{\alpha, \bar{\nu}}$; see Subsection 3.3 in [17. The trivial subbundle mentioned in the statement of the proposition is simply $\mathbb{C} \bar{\nu}$, if $\bar{\nu} \in \Gamma(\overline{\mathcal{S}} ; \mathcal{O})$ is generic and thus does not vanish.

We next prove Proposition 2.18B] The first step is to construct a section $\widetilde{s} \in \Gamma(\overline{\mathcal{S}} ; V)$ such that $\widetilde{s}=s$ outside of a small neighborhood of $\partial \overline{\mathcal{S}}$ that contains no zeros of $s$. This is achieved by cutting $s$ off near $\partial \overline{\mathcal{S}}$, so that the new section extends over $\partial \overline{\mathcal{S}}$ by zero. This procedure changes the estimate of $(1 \mathrm{~d})$ of Definition 2.14 in a well-controlled manner. We then add a small perturbation $t \nu$ to $\widetilde{s}$ such that $\widetilde{s}+t \nu$ has transverse zeros on $\mathcal{S}$ and no zeros on $\partial \overline{\mathcal{S}}$. The total number of zeros of this section is then the euler class of $V$ over $\overline{\mathcal{S}}$, $\langle e(V), \overline{\mathcal{S}}\rangle$. On the other hand, for each element of $s^{-1}(0)$ there will be a nearby zero of the perturbed section. All the remaining zeros will lie near $\partial \overline{\mathcal{S}}$. The final step is to show that all such zeros lie near the $s$-regular subsets of $\partial \overline{\mathcal{S}}$, for a good choice of $\nu$, and can be expressed in terms of the zeros of affine maps.

Let $\left\{\mathcal{Z}_{i}\right\}_{i \in I_{s}}$ be a collection of smooth manifolds in $\overline{\mathcal{M}}$ as in Definition 2.17] with corresponding semi-regularizations

$$
\left(U_{i}, E_{i}, \kappa_{i},\left(\mathcal{F}_{k_{i} ; j}\right)_{j \in J\left(\mathcal{Z}_{i}\right)} ; \mathcal{O}_{i}^{-} \oplus \mathcal{O}_{i}^{+}, \psi_{i}^{-} \oplus \psi_{i}^{+} ; \widetilde{\mathcal{F}}_{i}, \rho_{i}, \alpha_{i ;+}, \alpha_{i ; V}, \nu_{i}^{*}\right)
$$

By replacing $\mathcal{Z}_{i}$ with $\mathcal{Z}_{i} \cap U_{V}$, if necessary, it can be assumed that $\mathcal{Z}_{i} \subset U_{V}$. Let $\widetilde{\delta}_{i} \in C\left(\mathcal{Z}_{i} ; \mathbb{R}^{+}\right)$be such that $\vartheta_{\kappa_{i}}$ is defined on $E_{\widetilde{\delta}_{i}}$,

$$
\vartheta_{\kappa_{i} ; \mathcal{F}_{k_{i}}}\left(E_{\widetilde{\delta}_{i}} \times \mathcal{Z}_{i} \mathcal{F}_{k_{i} ; \tilde{\delta}_{i}}\right) \subset U_{k_{i}}, \quad \text { and } \quad \phi_{k_{i}} \vartheta_{\kappa_{i} ; \mathcal{F}_{k_{i}}}\left(E_{\widetilde{\delta}_{i}} \times \mathcal{Z}_{i} \mathcal{F}_{k_{i} ; \tilde{\delta}_{i}}\right) \subset U_{V}
$$

If $\delta \in C\left(\mathcal{Z}_{i} ; \mathbb{R}^{+}\right)$is less than $\widetilde{\delta}_{i}$ and $\mathcal{K}$ is a subset of $\mathcal{Z}_{i}$, let

$$
\begin{gathered}
\mathcal{U}_{i}(\delta)=E_{i ; \delta} \times \mathcal{Z}_{i} \mathcal{F}_{k_{i} ; \delta}-Y\left(E_{i} \oplus \mathcal{F}_{k_{i}} ; J\left(\mathcal{Z}_{i}\right)\right), \quad \text { and } \\
W_{i}(\delta ; \mathcal{K})=\phi_{k_{i}}\left(\vartheta_{\kappa_{i} ; \mathcal{F}_{k_{i}}}\left(E_{i ; \delta} \times \mathcal{K} \mathcal{F}_{k_{i} ; \delta}\right)\right) \subset \overline{\mathcal{M}}
\end{gathered}
$$

We denote $W_{i}\left(\delta ; \mathcal{Z}_{i}\right)$ by $W_{i}(\delta)$. Since all the zeros of $s$ are transverse, the next lemma implies that $s^{-1}(0)$ is a finite set. 
Lemma 2.19 There exists $\widetilde{\delta}_{i}^{*} \in C\left(\mathcal{Z}_{i} ; \mathbb{R}^{+}\right)$such that $W_{i}\left(\widetilde{\delta}_{i}^{*}\right) \cap s^{-1}(0)=\emptyset$.

Proof Let $\widetilde{\alpha}_{i}=\alpha_{i ;+} \oplus \alpha_{i ; V}$. By (1c), (2), and (3) of Definition 2.14 and (2) of Definition 2.15, there exists $\varepsilon_{\widetilde{\alpha}_{i}} \in C\left(\mathcal{Z}_{i} ; \mathbb{R}^{+}\right)$such that

$$
\left|\widetilde{\alpha}_{i}(b ; \widetilde{v})+\nu_{i}^{*}(b)\right| \geq \varepsilon_{\widetilde{\alpha}_{i}}(b)|\widetilde{v}| \quad \text { for all }(b ; \widetilde{v}) \in \widetilde{\mathcal{F}}_{i}
$$

If $\widetilde{\varepsilon}_{i ;+, V}$ is a $C^{0}$-negligible map as in (1d) of Definition 2.14 corresponding to $\mathcal{Z}_{i}$, let

$$
\begin{aligned}
\bar{\varepsilon}_{i ;+}(b, r)=\sup \left\{\left|\widetilde{\varepsilon}_{i ;+, V}(b ; w, v)\right|:(b ; w, v)\right. & \in E_{\widetilde{\delta}_{i}} \times_{\mathcal{Z}_{i}} \mathcal{F}_{k_{i} ;} ; \\
|w|,|v|<r, \quad(b ; v) & \left.\notin Y\left(\mathcal{F}_{k_{i}}, J\left(\mathcal{Z}_{i}\right)\right)\right\} .
\end{aligned}
$$

Then, $\bar{\varepsilon}_{i ;+}$ is continuous and $\lim _{r \longrightarrow 0} \bar{\varepsilon}_{i ;+}(b, r)=0$. Suppose

$$
(b ; w, v) \in \mathcal{U}_{i}(\delta), \quad \phi_{k_{i}} \vartheta_{\kappa_{i} ; \mathcal{F}_{k_{i}}}(b ; w, v) \in \mathcal{S}, \quad \text { and } \quad s\left(\phi_{k_{i}} \vartheta_{\kappa_{i} ; \mathcal{F}_{k_{i}}}(b ; w, v)\right)=0,
$$

where $\delta \leq \widetilde{\delta}_{i}$. Then, by Definition 2.14,

$$
\left|\widetilde{\alpha}_{i} \rho_{i}(b ; v)+\nu_{i}^{*}(b)\right| \leq \bar{\varepsilon}_{i ;+}(b, \delta(b))\left|\rho_{i}(b ; v)\right| .
$$

By (2.18) and (2.19), if $\bar{\varepsilon}_{i ;+}(b, \delta(b))<\varepsilon_{\widetilde{\alpha}_{i}}(b)$ for all $b \in \mathcal{Z}_{i}$, then $W_{i}(\delta) \cap s^{-1}(0)$ is empty.

Choose $\nu \in \Gamma(\overline{\mathcal{S}} ; V)$ with the following properties:

(A1) for all $i \in I_{s},\left.\bar{\nu}_{i} \equiv \nu\right|_{\mathcal{Z}_{i}}$ has no zeros, and the map

$$
\psi_{i ; \bar{\nu}_{i}}: \widetilde{\mathcal{F}}_{i} \longrightarrow \mathcal{O}_{i}^{+} \oplus V, \quad \psi_{i ; \bar{\nu}_{i}}(b ; v)=\left(\alpha_{i ;+}(v), \bar{\nu}_{i}(b)+\alpha_{i ; V}(v)\right),
$$

is transversal to the zero set. Furthermore, if $\mathcal{Z}_{i}$ is $s$-hollow, the sets $\mathbb{R} \nu_{i}^{*}$ and $\mathbb{R}^{*} \cdot \operatorname{Im} \psi_{i ; \bar{\nu}_{i}}(b ; v)$ are disjoint.

(A2) If $\mathcal{Z}_{i}$ is $s$-regular or $s$-neutral, $\psi_{i ; \bar{\nu}_{i}} \mid Y\left(\widetilde{\mathcal{F}}_{i} ; j\right)$ does not vanish for all $j \in \widetilde{I}\left(\mathcal{Z}_{i}\right)$;

(A3) If $\mathcal{Z}_{i}$ is $s$-regular, $\psi_{i ; \bar{\nu}_{i}}^{-1}(0)$ is a finite set.

Note that (A1) and (A2) are just transversality assumptions, due to (1c) and (3) of Definition 2.14 and (2) of Definition 2.15. Conditions (A3) holds if $\bar{\nu}_{i}$ is the restriction of a section $\bar{\nu}^{\prime} \in \Gamma\left(\overline{\mathcal{Z}}_{i} ; V\right)$ such that

$$
\left.\pi_{\widetilde{\mathcal{F}}_{i}}^{*} \bar{\nu}^{\prime} \in \Gamma_{\widetilde{\alpha}_{i ; V}} \overline{\left(\overline{\widetilde{\alpha}_{i ;+}^{-1}(0)}\right.} ; \pi_{\widetilde{\mathcal{F}}_{i}}^{*} V\right) .
$$


Lemma 2.20 If $\mathcal{Z}_{i}$ is $s$-hollow, there exists $\delta_{i}^{*} \in C\left(\mathcal{Z}_{i} ; \mathbb{R}^{+}\right)$with the following property. If $W$ is an open neighborhood of $\partial \overline{\mathcal{S}}$ in $\overline{\mathcal{M}}$, there exists $\epsilon>0$ such that for all $\eta \in C(\mathcal{S} ; \mathbb{R}), t \in \mathbb{R}^{+}$, and $\nu^{\prime} \in \Gamma(\mathcal{S} ; V)$, satisfying $\left.\nu^{\prime}\right|_{W}=\left.\nu\right|_{W}$ and $\left\|\nu-\nu^{\prime}\right\|_{C^{2}(\mathcal{S}-W)}<\epsilon$,

$$
W_{i}\left(\delta_{i}^{*}\right) \cap\left\{\eta s+t \nu^{\prime}\right\}^{-1}(0)=\emptyset .
$$

Proof By assumption (A1), the map

$$
\widetilde{\mathcal{F}}_{i} \oplus \mathbb{R} \longrightarrow \mathcal{O}_{i}^{+} \oplus V, \quad(b ; v) \longrightarrow\left(\alpha_{i ;+}(b ; v), \bar{\nu}_{i}(b)+\alpha_{i ; V}(v)\right)+\tau \nu_{i}^{*}(b),
$$

has no zeros over $\mathcal{Z}_{i}$. Thus, there exists $\delta_{\widetilde{\alpha}_{i}, \bar{\nu}_{i}} \in C\left(\mathcal{Z}_{i} ; \mathbb{R}^{+}\right)$such that

$$
\left|\left(0, \bar{\nu}_{i}(b)\right)+\widetilde{\alpha}_{i}(b ; \widetilde{v})+\tau \nu_{i}^{*}(b)\right| \geq \delta_{\widetilde{\alpha}_{i}, \bar{\nu}_{i}}(b)\left(1+\left|\widetilde{\alpha}_{i}(b ; \widetilde{v})\right|\right) \quad \text { for }(b ; \widetilde{v}) \in \widetilde{\mathcal{F}}_{i} .
$$

By continuity of $\nu$, there exists $\varepsilon_{i, \nu} \in C^{0}\left(\mathcal{Z}_{i} \times \mathbb{R} ; \mathbb{R}\right)$ such that

$$
\lim _{r \longrightarrow 0} \varepsilon_{i, \nu}(b, r)=0 \quad \text { and } \quad\left|\nu\left(\phi_{k_{i}} \vartheta_{\kappa_{i} ; \mathcal{F}_{k_{i}}}(b ; w, v)\right)-\bar{\nu}_{i}(b)\right| \leq \varepsilon_{i, \nu}(b, \delta(b))
$$

for all $(b ; w, v) \in \mathcal{U}_{i}(\delta)$ such that $\phi_{k_{i}} \vartheta_{\kappa_{i} ; \mathcal{F}_{k_{i}}}(b ; w, v) \in \mathcal{S}$. If $\nu^{\prime} \in \Gamma(\mathcal{S} ; V)$ is such that $\left.\nu^{\prime}\right|_{W}=\left.\nu\right|_{W}$ and $\left\|\nu-\nu^{\prime}\right\|_{C^{2}(\mathcal{S}-W)}<\epsilon$, then

$$
\left|\left\{\nu-\nu^{\prime}\right\}\left(\phi_{k_{i}} \vartheta_{\kappa ; \mathcal{F}_{k_{i}}}(b ; w, v)\right)\right| \leq C_{i, W}(b) \delta(b) \epsilon
$$

for all $(b ; w, v) \in \mathcal{U}_{i}(\delta)$ such that $\phi_{k_{i}} \vartheta_{\kappa_{i} ; \mathcal{F}_{k_{i}}}(b ; w, v) \in \mathcal{S}$, where $C_{i, W} \in C\left(\mathcal{Z}_{i} ; \mathbb{R}\right)$ depends only on $W$. Thus, by the above and (1d) of Definition 2.14.

$$
\begin{aligned}
\mid\left(0, t \bar{\nu}_{i}(b)\right)+ & +\widetilde{\alpha}_{i}\left(\eta\left(\phi_{k_{i}} \vartheta_{\kappa_{i} ; \mathcal{F}_{k_{i}}}(b ; w, v)\right) \rho_{i}(b ; v)\right)+\eta\left(\phi_{k_{i}} \vartheta_{\kappa_{i} ; \mathcal{F}_{k_{i}}}(b ; w, v)\right) \nu_{i}^{*}(b) \mid \\
\leq & \varepsilon_{\widetilde{\alpha}_{i}}(b)^{-1} \bar{\varepsilon}_{+}(b, \delta(b))\left|\widetilde{\alpha}_{i}\left(\eta\left(\phi_{k_{i}} \vartheta_{\kappa_{i} ; \mathcal{F}_{k_{i}}}(b ; w, v)\right) \rho_{i}(b ; v)\right)\right| \\
& +t\left(\varepsilon_{i, \nu}(b, \delta(b))+C_{i, W}(b) \delta(b) \epsilon\right)
\end{aligned}
$$

for all $(b ; w, v) \in \mathcal{U}_{i}(\delta)$ such that $\phi_{k_{i}} \vartheta_{\kappa_{i} ; \mathcal{F}_{k_{i}}}(b ; w, v) \in \mathcal{S}$ and

$$
\left\{\eta s+t \nu^{\prime}\right\} \phi_{k_{i}} \vartheta_{\kappa_{i} ; \mathcal{F}_{k_{i}}}(b ; w, v)=0,
$$

where $\varepsilon_{\widetilde{\alpha}_{i}}$ is as in the proof of Lemma 2.19. By (2.20) and (2.21),

$$
W_{i}(\delta) \cap\left\{\eta s+t \nu^{\prime}\right\}^{-1}(0)=\emptyset
$$

if $\varepsilon_{\widetilde{\alpha}_{i}}(b)^{-1} \bar{\varepsilon}_{+}(b, \delta(b)), \varepsilon_{i, \nu}(b, \delta(b)), C_{i, W}(b) \delta(b) \epsilon<\frac{1}{4} \delta_{\widetilde{\alpha}_{i}, \bar{\nu}_{i}}(b)$ for all $b \in \mathcal{Z}_{i}$.

Lemma 2.21 If $\mathcal{Z}_{i}$ is $s$-neutral, there exists $\delta_{i}^{*} \in C\left(\mathcal{Z}_{i} ; \mathbb{R}^{+}\right)$with the following property. If $W$ is an open neighborhood of $\partial \overline{\mathcal{S}}$ in $\overline{\mathcal{M}}$, there exists $\epsilon>0$ such that for all $\eta \in C(\mathcal{S} ; \mathbb{R}), t \in \mathbb{R}^{+}$, and $\nu^{\prime} \in \Gamma(\mathcal{S} ; V)$, satisfying $\left.\nu^{\prime}\right|_{W}=\left.\nu\right|_{W}$ and $\left\|\nu-\nu^{\prime}\right\|_{C^{2}(\mathcal{S}-W)}<\epsilon$,

$$
W_{i}\left(\delta_{i}^{*}\right) \cap\left\{\eta s+t \nu^{\prime}\right\}^{-1}(0)=\emptyset .
$$


Proof By assumptions (A1) and (A2), $\psi_{i, \bar{\nu}_{i}}^{-1}(0)$ is a discrete subset of $\widetilde{\mathcal{F}}_{i}-$ $Y\left(\widetilde{\mathcal{F}}_{i} ; \widetilde{I}\left(\mathcal{Z}_{i}\right)\right)$ and contains at most one point of each fiber. For each $\widetilde{v} \in \psi_{i, \bar{\nu}_{i}}^{-1}(0)$, let $\mathcal{K}_{\widetilde{v}}$ be a neighborhood of $\widetilde{v}$ in $\widetilde{\mathcal{F}}_{i}-Y\left(\widetilde{\mathcal{F}}_{i} ; \widetilde{I}\left(\mathcal{Z}_{i}\right)\right)$ such that the closure of $\mathcal{K}_{\widetilde{v}}$ in $\widetilde{\mathcal{F}}_{i}-Y\left(\widetilde{\mathcal{F}}_{i} ; \widetilde{I}\left(\mathcal{Z}_{i}\right)\right)$ is compact. For simplicity, it can be assumed that all the sets $\mathcal{K}_{\tilde{v}}$ are disjoint. By Proposition 2.3A there exists $\delta_{\rho_{i}, \bar{\nu}_{i}} \in C^{\infty}\left(\mathcal{S} ; \mathbb{R}^{+}\right)$ such that

$$
\mathcal{F}_{k_{i} ; \delta_{\rho_{i}, \bar{\nu}_{i}}} \cap \rho_{i}^{-1}\left(\bigcup_{\widetilde{v} \in \psi_{i, \bar{\nu}_{i}}^{-1}(0)} \mathbb{R}^{+} \cdot \mathcal{K}_{\widetilde{v}}\right)=\emptyset .
$$

Then, there exists $\delta_{\bar{\nu}_{i}} \in C\left(\mathcal{Z}_{i} ; \mathbb{R}^{+}\right)$such that for all $\tau \in \mathbb{R}$

$$
\left|\left(0, \bar{\nu}_{i}(b)\right)+\widetilde{\alpha}_{i}\left(\tau \rho_{i}(b ; v)\right)\right| \geq \delta_{\bar{\nu}_{i}}(b)\left(1+\left|\widetilde{\alpha}_{i}\left(\tau \rho_{i}(b ; v)\right)\right|\right)
$$

for all $(b ; v) \in \mathcal{F}_{k_{i} ; \delta_{\rho_{i}, \bar{\nu}_{i}}}-Y\left(\widetilde{\mathcal{F}}_{i} ; \widetilde{I}\left(\mathcal{Z}_{i}\right)\right)$. On the other hand, by the same argument as in the proof of Lemma 2.20

$$
\begin{aligned}
\mid\left(0, t \bar{\nu}_{i}(b)\right)+\widetilde{\alpha}_{i}( & \left.\eta(b ; w, v) \rho_{i}(b ; v)\right) \mid \\
\leq \varepsilon_{\widetilde{\alpha}_{i}}(b)^{-1} \bar{\varepsilon}_{+}(b, \delta(b)) \mid & \left|\widetilde{\alpha}_{i}\left(\eta(b ; w, v) \rho_{i}(b ; v)\right)\right| \\
& +t\left(\varepsilon_{i, \nu}(b, \delta(b))+C_{i, W}(b) \delta(b) \epsilon\right)
\end{aligned}
$$

for all $(b ; w, v) \in \mathcal{U}_{i}(\delta)$ such that $\phi_{k_{i}} \vartheta_{\kappa_{i} ; \mathcal{F}_{k_{i}}}(b ; w, v) \in \mathcal{S}$ and

$$
\left\{\eta s+t \nu^{\prime}\right\} \phi_{k_{i}}(b ; w, v)=0,
$$

if $\left.\nu^{\prime}\right|_{W}=\left.\nu\right|_{W}$ and $\left\|\nu-\nu^{\prime}\right\|_{C^{2}(\mathcal{S}-W)}<\epsilon$. Thus, $W_{i}(\delta) \cap\left\{\eta s+t \nu^{\prime}\right\}^{-1}(0)=\emptyset$ if $\varepsilon_{\widetilde{\alpha}_{i}}(b)^{-1} \bar{\varepsilon}_{+}(b, \delta(b)), \varepsilon_{i, \nu}(b, \delta(b)), C_{i, W}(b) \delta(b) \epsilon<\frac{1}{4} \delta_{\bar{\nu}_{i}}(b)$ for all $b \in \mathcal{Z}_{i}$ and $\delta \leq \delta_{\rho_{i}, \bar{\nu}_{i}}$.

Lemma 2.22 If $\mathcal{Z}_{i}$ is $s$-regular, there exist $\delta_{i}^{*} \in C\left(\mathcal{Z}_{i} ; \mathbb{R}^{+}\right)$, a compact subset $\mathcal{K}_{\tilde{\alpha}_{i} ; \bar{\nu}_{i}}$ of $\mathcal{Z}_{i}$, and $\delta_{i}, \epsilon_{i} \in \mathbb{R}^{+}$with the following property. If $W$ is an open neighborhood of $\partial \overline{\mathcal{S}}$ in $\overline{\mathcal{M}}$, there exists $\epsilon_{W} \in \mathbb{R}^{+}$such that for every $\eta \in$ $C(\mathcal{S} ; \mathbb{R})$, satisfying

$$
\eta\left(\phi_{k_{i}} \vartheta_{\kappa_{i} ; \mathcal{F}_{k_{i}}}(w, v)\right)=\beta_{\delta_{i}}(|v|)
$$

for all $(w, v) \in E_{i ; \delta_{i}^{*}} \times_{\mathcal{K}_{\tilde{\alpha}_{i} ; \bar{\nu}_{i}}} \mathcal{F}_{k_{i} ; \delta_{i}^{*}}$ such that $\phi_{k_{i}} \vartheta_{\kappa_{i} ; \mathcal{F}_{k_{i}}}(w, v) \in \mathcal{S}, t \in\left(0, \epsilon_{i}\right)$, and $\nu^{\prime} \in \Gamma(\mathcal{S} ; V)$, satisfying $\left.\nu^{\prime}\right|_{W}=\left.\nu\right|_{W},\left\|\nu-\nu^{\prime}\right\|_{C^{2}(\mathcal{S}-W)}<\epsilon_{W}$, and $\eta s+t \nu^{\prime}$ is transversal to the zero set on $\mathcal{S}$, then

$$
{ }^{ \pm}\left|W_{i}\left(\delta_{i}^{*}\right) \cap\left\{\eta s+t \nu^{\prime}\right\}^{-1}(0)\right|=\operatorname{deg} \rho_{i} \cdot{ }^{ \pm}\left|\psi_{i, \bar{\nu}}^{-1}(0)\right|
$$

and

$$
W_{i}\left(\delta_{i}^{*}\right) \cap\left\{\eta s+t \nu^{\prime}\right\}^{-1}(0) \subset W_{i}\left(\delta_{i}^{*} ; K_{\tilde{\alpha}_{i} ; \bar{\nu}_{i}}\right) .
$$

Furthermore, if $W^{*}$ is a neighborhood of $\mathcal{Z}_{i}$ in $\overline{\mathcal{M}}, \delta_{i}^{*}$ can be chosen so that $W_{i}\left(\delta_{i}\right) \subset W^{*}$. 
Proof (1) Let $\mathcal{K}$ be an open neighborhood of the finite subset $\psi_{i, \bar{\nu}_{i}}^{-1}(0)$ of

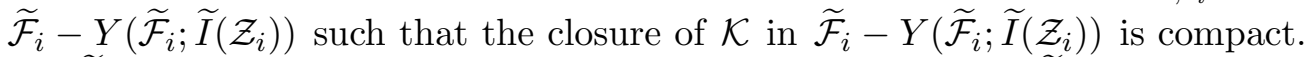
Let $\widetilde{\mathcal{K}}_{\widetilde{\alpha}_{i}, \bar{\nu}_{i}}=\rho_{i}^{-1}(\mathbb{R} \cdot \overline{\mathcal{K}})$. Note that by (1) of Proposition 2.3B,$\widetilde{\mathcal{K}}_{\widetilde{\alpha}_{i}, \bar{\nu}_{i}}$ is a closed subset of $\mathcal{F}_{k_{i}}-\mathcal{Z}_{i}$. We take

$$
\mathcal{K}_{\widetilde{\alpha}_{i}, \bar{\nu}_{i}}=\pi_{\mathcal{F}_{k_{i}}}\left(\widetilde{\mathcal{K}}_{\widetilde{\alpha}_{i}, \bar{\nu}_{i}}\right)=\pi_{\widetilde{\mathcal{F}}_{i}}(\overline{\mathcal{K}}) .
$$

This is a compact subset of $\mathcal{Z}_{i}$.

(2) As in the proofs of Lemmas 2.20 and 2.21 there exists $\delta_{\bar{\nu}_{i}} \in C\left(\mathcal{Z}_{i} ; \mathbb{R}^{+}\right)$ such that

$$
\left|\left(0, \bar{\nu}_{i}(b)\right)+\widetilde{\alpha}_{i}(b ; \widetilde{v})\right|>\delta_{\bar{\nu}_{i}}(b)\left(1+\left|\widetilde{\alpha}_{i}(b ; \widetilde{v})\right|\right) \quad \text { for all }(b ; \widetilde{v}) \in \widetilde{\mathcal{F}}_{i}-\mathbb{R} \cdot \mathcal{K} .
$$

On the other hand, as before, by the estimate (1d) of Definition 2.14.

$$
\begin{aligned}
& \left|\left(0, t \bar{\nu}_{i}(b)\right)+\widetilde{\alpha}_{i}\left(\eta(b ; w, v) \rho_{i}(b ; v)\right)\right| \\
& \quad \leq \widetilde{\varepsilon}_{+}\left(b, \delta_{i}^{*}(b)\right)\left|\widetilde{\alpha}_{i}\left(\eta(b ; w, v) \rho_{i}(b ; v)\right)\right|+t\left(\varepsilon_{i, \nu}\left(b, \delta_{i}^{*}(b)\right)+\widetilde{C}_{i, W}(b) \epsilon_{W}\right)
\end{aligned}
$$

for all $(b ; w, v) \in \mathcal{U}_{i}\left(\delta_{i}^{*}\right)$ such that $\phi_{k_{i}} \vartheta_{\kappa_{i} ; \mathcal{F}_{k_{i}}}(b ; w, v) \in \mathcal{S}$ and

$$
\left\{\eta s+t \nu^{\prime}\right\}(b ; w, v)=0,
$$

where $\widetilde{\varepsilon}_{+}\left(b, \delta_{i}^{*}(b)\right)=\varepsilon_{\widetilde{\alpha}_{i}}(b)^{-1} \bar{\varepsilon}_{+}\left(b, \delta_{i}^{*}(b)\right)$ and $\widetilde{C}_{i, W}(b)=C_{i, W}(b) \delta_{i}^{*}(b)$. By (2.24) and (2.25),

$$
\begin{aligned}
& \quad W_{i}\left(\delta_{i}^{*}\right) \cap\left\{\eta s+t \nu^{\prime}\right\}^{-1}(0) \\
& \qquad \subset \phi_{k_{i}} \vartheta_{\kappa_{i} ; \mathcal{F}_{k_{i}}}\left(\left\{(w, v) \in E_{i} \oplus \mathcal{F}_{k_{i}}: v \in \widetilde{\mathcal{K}}_{\widetilde{\alpha}_{i}, \bar{\nu}_{i}},|w|,|v|<\delta_{i}^{*}(b)\right\}\right) \\
& \text { if } \widetilde{\varepsilon}_{+}\left(b, \delta_{i}^{*}(b)\right), \varepsilon_{i, \nu}\left(b, \delta_{i}^{*}(b)\right), \widetilde{C}_{i, W}(b) \epsilon_{W}<\frac{1}{4} \delta_{\bar{\nu}_{i}}(b) \text { for all } b \in \mathcal{Z}_{i} .
\end{aligned}
$$

(3) Let $\widetilde{\rho}_{i} \equiv\left(\widetilde{\rho}_{i, i^{\prime}}\right)_{i^{\prime} \in I\left(\mathcal{Z}_{i}\right)}$ and $\alpha_{i ;-}$ be the monomials map and the vectorbundle isomorphism as in (3) and (4) of Definition 2.15 respectively, corresponding to $\mathcal{Z}_{i}$. Similarly, for each $i^{\prime} \in I\left(\mathcal{Z}_{i}\right)$, let $\widetilde{\varepsilon}_{i, i^{\prime}}^{-}$and $\varepsilon_{i, i^{\prime}}^{-}$be a $C^{1-}$ negligible map and a $\left(\rho_{i}, \widetilde{\rho}_{i, i^{\prime}}\right)$-controlled map as in (5) of Definition 2.15] corresponding to $\mathcal{Z}_{i}$. Since $\alpha_{i ;-}$ is an isomorphism on every fiber and the image of $\widetilde{\varepsilon}_{i, i^{\prime}}^{-}$lies in the subbundle $\alpha_{i ;-}\left(E_{i, i^{\prime}}\right)$ of $\mathcal{O}^{-}$, we can define a $C^{1}$-negligible map

$$
\widetilde{\varepsilon}_{i, i^{\prime}}: \mathcal{F}_{k_{i}} \mid \mathcal{U}_{i}-Y\left(\mathcal{F}_{k_{i}} \mid \mathcal{U}_{i} ; J\left(\mathcal{Z}_{i}\right)\right) \longrightarrow E_{i}
$$

by $\widetilde{\varepsilon}_{i, i^{\prime}}^{-}(w, v)=\alpha_{i ;-}\left(\widetilde{\varepsilon}_{i, i^{\prime}}(w, v) \otimes \widetilde{\rho}_{i, i^{\prime}}(v)\right)$. By the Contraction Principle, there exists $\delta_{-} \in \mathbb{R}^{+}$such that for all

$$
\left(w^{\prime}, v\right) \in E_{i ; \delta_{-}} \times_{\mathcal{K}_{\tilde{\alpha}_{i}, \bar{\nu} i}} \mathcal{F} \mathcal{T}_{k_{i}, \delta_{-}}
$$


the equation

$$
w+\sum_{i^{\prime} \in I\left(\mathcal{Z}_{i}\right)} \widetilde{\varepsilon}_{i, i^{\prime}}(w, v)=w^{\prime}, \quad w \in E_{i ; 2 \delta_{-}},
$$

has a unique solution. Furthermore, this solution satisfies $|w| \leq 2\left|w^{\prime}\right|$; see the proof of [17. Lemma 3.18], for example. Let $\epsilon_{-} \in \mathbb{R}^{+}$be such that

$$
\left|\alpha_{i ;-}(\widetilde{w})\right| \geq \epsilon_{-}|\widetilde{w}| \quad \text { for all } \widetilde{w} \in \widetilde{E} \mid \mathcal{K}_{\widetilde{\alpha}_{i}, \bar{\nu}_{i}} .
$$

By (3) of Definition 2.13 and (3) of Proposition 2.3B there exists $\delta_{+} \in \mathbb{R}^{+}$ such that

$$
\left|\varepsilon_{i, i^{\prime}}^{-}(w, v)\right| \leq \frac{\epsilon_{-} \delta_{-}}{4\left|I\left(\mathcal{Z}_{i}\right)\right|}\left|\widetilde{\rho}_{i, i^{\prime}}(v)\right|
$$

for all $i^{\prime} \in I\left(\mathcal{Z}_{i}\right)$ and $v \in \widetilde{\mathcal{K}}_{\widetilde{\alpha}_{i}, \bar{\nu}_{i}}$ such that $|v| \leq \delta_{+}$. It can be assumed that $\delta_{+} \leq \delta_{-}$. Then by (5) of Definition 2.15,

$$
\left|\alpha_{i ;-}\left(\left(w+\widetilde{\varepsilon}_{i, i^{\prime}}(w, v)\right) \otimes \widetilde{\rho}_{i, i^{\prime}}(v)\right)\right|<\frac{\epsilon_{-} \delta_{-}}{2\left|I\left(\mathcal{Z}_{i}\right)\right|}\left|\widetilde{\rho}_{i, i^{\prime}}(v)\right|
$$

for all $(w, v) \in E_{i ; \delta_{i}^{*}} \times \mathcal{Z}_{i} \mathcal{F}_{k_{i} ; \delta_{i}^{*}}$ where $v \in \widetilde{\mathcal{K}}_{\tilde{\alpha}_{i}, \bar{\nu}_{i}},|v| \leq \delta_{+}, \quad \phi_{k_{i}} \vartheta_{\kappa_{i} ; \mathcal{F}_{k_{i}}}(w, v) \in \mathcal{S}$.

(4) Let $\delta_{i}=\frac{1}{4} \delta_{+}^{2}$. If $\delta_{+}<\inf _{\mathcal{K}_{\tilde{\alpha}_{i}, \bar{\nu}_{i}}} \delta_{i}^{*}$, by (2) of Proposition 2.3B there exists $\epsilon_{i} \in \mathbb{R}^{+}$such that for all $t \in\left(0, \epsilon_{i}\right)$,

$$
\mathcal{F}_{k_{i} ; \delta_{i}^{*}} \cap\left\{t^{-1} \eta \rho_{i}\right\}^{-1}(\mathcal{K}) \subset \mathcal{F}_{k_{i}, \delta_{+}},
$$

and $t^{-1} \eta \rho_{i}: \mathcal{F}_{k_{i} ; \delta_{i}^{*}} \cap\left\{t^{-1} \eta \rho_{i}\right\}^{-1}(\mathcal{K}) \longrightarrow \mathcal{K}$ is a covering projection of oriented degree $\operatorname{deg} \rho_{i}$. Then, by (2.26) -(2.28) and the assumption on $\delta_{-}$,

$$
\begin{aligned}
W_{i}\left(\delta_{i}^{*}\right) \cap & \left\{\eta s+t \nu^{\prime}\right\}^{-1}(0) \\
& \subset \phi_{k_{i}} \vartheta_{\kappa_{i} ; \mathcal{F}_{k_{i}}}\left(\left\{(b ; w, v) \in E_{i} \oplus \mathcal{F}_{k_{i}}: b \in \mathcal{K}_{\widetilde{\alpha}_{i}, \bar{\nu}_{i}},|w|,|v| \leq \frac{1}{2} \delta_{i}^{*}(b)\right\}\right)
\end{aligned}
$$

for all $t \in\left(0, \epsilon_{i}\right)$, if $\delta_{ \pm} \leq \frac{1}{2} \delta_{i}^{*}(b)$ for all $b \in \mathcal{K}_{\widetilde{\alpha}_{i}, \bar{\nu}_{i}}$ and

$$
\widetilde{\varepsilon}_{+}\left(b, \delta_{i}^{*}(b)\right), \varepsilon_{i, \nu}\left(b, \delta_{i}^{*}(b)\right), \widetilde{C}_{i, W}(b) \epsilon_{W}<\frac{1}{4} \delta_{\bar{\nu}_{i}}(b)
$$

for all $b \in \mathcal{Z}_{i}$.

(5) By Definition 2.14, the set $W_{i}\left(\delta_{i}^{*}\right) \cap\left\{\eta s+t \nu^{\prime}\right\}^{-1}(0)$ consists of the solutions of the system

$$
\left\{\begin{aligned}
\psi_{i}^{-}(w, v) & =0 \in \mathcal{O}^{-} \\
\psi_{i}^{+}(w, v) & =0 \in \mathcal{O}^{+} \quad(w, v) \in \mathcal{U}_{i}\left(\delta_{i}^{*}\right) . \\
\left\{\eta s+t \nu^{\prime}\right\}(w, v) & =0 \in V
\end{aligned}\right.
$$


By our assumptions, the zeros of this system of equations are transverse. By (1)-(4) and Definitions 2.14 and 2.15] these zeros are the same as the zeros of the system

$$
\left\{\begin{aligned}
\alpha_{i ;-} \widetilde{\rho}_{i}(w, v)+\sum_{i^{\prime} \in I\left(\mathcal{Z}_{i}\right)}\left(\widetilde{\varepsilon}_{i, i^{\prime}}^{-}(w, v) \widetilde{\rho}_{i, i^{\prime}}(v)+\varepsilon_{i, i^{\prime}}^{-}(w, v)\right) & =0 \in \mathcal{O}^{-} \\
\beta_{\delta_{i}}(|v|)\left(\alpha_{i ;+}+\widetilde{\varepsilon}_{i ;+}(w, v)\right) \rho_{i}(v) & =0 \in \mathcal{O}^{+} \\
\beta_{\delta_{i}}(|v|)\left(\alpha_{i ; V}+\widetilde{\varepsilon}_{i ; V}(w, v)\right) \rho_{i}(v)+t \nu^{\prime}(w, v) & =0 \in V
\end{aligned}\right.
$$

where $\widetilde{\varepsilon}_{i ;+}$ and $\widetilde{\varepsilon}_{i ; V}$ denote the $\mathcal{O}^{+}$and $V$-components of $\widetilde{\varepsilon}_{i ;+, V}$ and $(w, v) \in$ $\mathcal{U}_{i}\left(\delta_{i}^{*}\right)$ as before. We will show that the zeros of (2.29) are cobordant to the zeros of the system

$$
\left\{\begin{array}{rl}
\alpha_{i ;-} \widetilde{\rho}_{i}(b ; w, v) & =0 \in \mathcal{O}^{-} \\
\alpha_{i ; V}\left(\beta_{\delta_{i}}(|v|) \rho_{i}(b ; v)\right) & =0 \in \mathcal{O}^{+} \\
\alpha_{i ;+}\left(\beta_{\delta_{i}}(|v|) \rho_{i}(b ; v)\right)+t \bar{\nu}_{i}(b) & =0
\end{array} \quad(b ; w, v) \in \mathcal{U}_{i}\left(\delta_{i}^{*}\right) .\right.
$$

We construct a cobordism between the two zero sets as follows. If $h_{-}, h_{+}$, and $h_{V}$ are bundle maps from $\mathcal{X} \equiv[0,1] \times \mathcal{U}_{i}\left(\delta_{i}^{*}\right)$ to $\mathcal{O}^{-}, \mathcal{O}^{+}$, and $V$, respectively, and $\underline{h}=\left(h_{-}, h_{+}, h_{V}\right)$, let $\mathcal{X}(\underline{h})$ be the set ot tuples $(\tau,(b ; w, v)) \in \mathcal{X}$ such that

$$
\left\{\begin{aligned}
& \beta_{\delta_{i}}(|v|)\left(\alpha_{i ;+}+\tau \widetilde{\varepsilon}_{i ;+}(b ; w, v)\right) \rho_{i}(b ; v)+h_{+}(\tau, b ; w, v)=0 \\
& \beta_{\delta_{i}}(|v|)\left(\alpha_{i ; V}+\tau \widetilde{\varepsilon}_{i ; V}(b ; w, v)\right) \rho_{i}(b ; v)+t\left(\tau \nu^{\prime}(b ; w, v)\right.\left.+(1-\tau) \bar{\nu}_{i}(b)\right) \\
&+h_{V}(\tau, b ; w, v)=0 \\
& \alpha_{i ;-} \widetilde{\rho}_{i}(b ; w, v)+\tau \sum_{i^{\prime} \in I\left(\mathcal{Z}_{i}\right)}\left(\widetilde{\varepsilon}_{i, i^{\prime}}^{-}(b ; w, v) \widetilde{\rho}_{i, i^{\prime}}(b ; v)+\varepsilon_{i, i^{\prime}}^{-}(b ; w, v)\right) \\
&+h_{-}(\tau, b ; w, v)=0 .
\end{aligned}\right.
$$

Since the zeros of (2.29) and (2.30) are transverse, for a generic choice of $\underline{h}$ with the boundary condition $\underline{h}_{\tau=0,1} \equiv 0, \mathcal{X}(\underline{h})$ is a smooth oriented manifold such that $\partial \mathcal{X}(\underline{h})=\mathcal{X}_{1}(\underline{h})-\mathcal{X}_{0}(\underline{h})$. By the same argument as in (1)-(4),

$$
\begin{aligned}
\mathcal{X}(\underline{h}) \subset[0,1] \times\left\{(b ; w, v) \in E_{i} \oplus \mathcal{F}_{k_{i}}:(b ; v)\right. & \in \widetilde{\mathcal{K}}_{\widetilde{\alpha}_{i}, \bar{\nu}_{i}}, \\
& \left.\delta_{i} \leq|v| \leq \frac{1}{2} \delta_{i}^{*}(b),|w|<\frac{1}{2} \delta_{i}^{*}(b)\right\},
\end{aligned}
$$

if $\left|h_{V}(\tau, b ; w, v)\right|,\left|h_{+}(\tau, b ; w, v)\right| \leq \frac{1}{3} t \delta_{\bar{\nu}_{i}}(b)$ for all $b \in \mathcal{Z}_{i}$ and $\left|h_{-}(\tau, b ; w, v)\right| \leq$ $\frac{\epsilon_{-} \delta_{-}}{4\left|I\left(\mathcal{Z}_{i}\right)\right|}\left|\widetilde{\rho}_{i, i^{\prime}}(v)\right|$ for all $v \in \widetilde{\mathcal{K}}_{\widetilde{\alpha}_{i}, \bar{\nu}_{i}}$ such that $\delta_{i} \leq|v| \leq \delta_{i}^{*}(b), i^{\prime} \in I\left(\mathcal{Z}_{i}\right)$. The lower bound on $|v|$ above follows from the fact that $\beta_{\delta_{i}}(|v|)$ is zero if $|v|<\delta_{i}$. Thus, $\mathcal{X}(\underline{h})$ is a compact space if $\underline{h}$ is sufficiently small. We conclude that

$$
{ }^{ \pm}\left|\mathcal{X}_{1}(\underline{h})\right|={ }^{ \pm}\left|\mathcal{X}_{0}(\underline{h})\right|=\operatorname{deg} \rho_{i} \cdot{ }^{ \pm}\left|\psi_{i, \bar{\nu}}^{-1}(0)\right| .
$$

The second equality is immediate from (A1), (A2), (A3), and (2) of Proposition 2.3B. This concludes the proof of the main claim of the lemma. The other claim is clear. 
Proposition 2.18B is essentially proved; it only remains to construct a cutoff function $\eta$ that has good properties. Let $W_{1}=\bigsqcup_{i \in I_{s}} W_{i}\left(\delta_{i}^{*} / 2\right)$. This is an open neighborhood of $\partial \overline{\mathcal{S}}$ in $\overline{\mathcal{M}}$. By Lemmas 2.19]2.22 it can be assumed that $s^{-1}(0) \cap \bar{W}_{1}=\emptyset$ and that

$$
\overline{\phi_{k_{i}}\left(\vartheta_{\kappa_{i} ; \mathcal{F}_{k_{i}}}\left(E_{i ; \delta_{i}^{*}} \times \mathcal{K}_{\tilde{\alpha}_{i}, \bar{\nu}_{i}} \mathcal{F}_{k_{i} ; \delta_{i}^{*}}\right)\right)} \cap \overline{\phi_{k_{j}}\left(\vartheta_{\kappa_{j} ; \mathcal{F}_{k_{j}}}\left(E_{j ; \delta_{j}^{*}} \times \mathcal{K}_{\tilde{\alpha}_{j}, \bar{\nu}_{j}} \mathcal{F}_{k_{j} ; \delta_{j}^{*}}\right)\right)}=\emptyset
$$

for all $i, j \in I_{s}^{*}$ such that $i \neq j$. Let $W_{0}$ be an open neighborhood of $\partial \overline{\mathcal{S}}$ in $\overline{\mathcal{M}}$ such that $\bar{W}_{0} \subset W_{1}$ and let $\eta^{\prime}: \mathcal{M} \longrightarrow[0,1]$ be a smooth function such that $\eta^{\prime} \mid W_{0}=0$ and $\eta^{\prime} \mid \mathcal{M}-W_{1}=1$. For each $i \in I_{s}^{*}$, let $\mathcal{K}_{\tilde{\alpha}_{i}, \bar{\nu}_{i}}^{\prime}$ be a compact subset of $\mathcal{Z}_{i}$ such that $\mathcal{K}_{\tilde{\alpha}_{i}, \bar{\nu}_{i}} \subset \operatorname{Int}_{\mathcal{Z}_{i}} \mathcal{K}_{\tilde{\alpha}_{i}, \bar{\nu}_{i}}^{\prime}$ and

$$
\overline{\phi_{k_{i}}\left(\vartheta_{\kappa_{i} ; \mathcal{F}_{k_{i}}}\left(E_{i ; \delta_{i}^{*}} \times_{\mathcal{K}_{\tilde{\alpha}_{i}, \bar{\nu}_{i}}^{\prime}} \mathcal{F}_{k_{i} ; \delta_{i}^{*}}\right)\right)} \cap \overline{\phi_{k_{j}}\left(\vartheta_{\kappa_{j} ; \mathcal{F}_{k_{j}}}\left(E_{j ; \delta_{j}^{*}} \times{ }_{\mathcal{K}_{\tilde{\alpha}_{j}, \bar{\nu}_{j}}^{\prime}} \mathcal{F}_{k_{j} ; \delta_{j}^{*}}\right)\right)}=\emptyset
$$

for all $i, j \in I_{s}^{*}$ such that $i \neq j$. Choose a smooth function $\eta_{i}^{\prime}: E_{i} \oplus \mathcal{F}_{k_{i}} \longrightarrow[0,1]$ such that

$$
\eta_{i}^{\prime} \mid E_{i ; \delta_{i}^{*} / 2} \times \mathcal{K}_{\tilde{\alpha}_{i}, \bar{\nu}_{i}} \mathcal{F}_{k_{i} ; \delta_{i}^{*} / 2}=1 \quad \text { and } \quad \eta_{i}^{\prime} \mid\left(E_{i} \oplus \mathcal{F}_{k_{i}}-E_{i ; \delta_{i}^{*}} \times_{\mathcal{K}_{\tilde{\alpha}_{i}, \bar{\nu}_{i}}^{\prime}} \mathcal{F}_{k_{i} ; \delta_{i}^{*}}\right)=0 \text {. }
$$

If $\delta_{i} \in \mathbb{R}^{+}$is as in Lemma 2.22 we define $\eta_{i}: \mathcal{S} \longrightarrow[0,1]$ by

$$
\eta_{i}\left(b^{*}\right)=\eta_{i}^{\prime}(b ; w, v) \beta_{\delta_{i}}(|v|)+\left(1-\eta_{i}^{\prime}(b ; w, v)\right) \eta^{\prime}\left(b^{*}\right)
$$

if $b^{*}=\phi_{k_{i}} \vartheta_{\kappa_{i} ; \mathcal{F}_{k_{i}}}(b ; w, v)$ for some $(b ; w, v) \in E_{i ; \delta_{i}^{*}} \times_{\mathcal{K}_{\tilde{\alpha}_{i}, \bar{\nu}_{i}}^{\prime}} \mathcal{F}_{k_{i} ; \delta_{i}^{*}}$, and

$$
\eta_{i}\left(b^{*}\right)=\eta^{\prime}\left(b^{*}\right)
$$

otherwise. This function is smooth on $\mathcal{S}$, since it is the restriction of a smooth function on $\mathcal{M}$. Let

$$
\eta=\eta^{\prime}+\sum_{i \in I_{s}^{*}} \eta_{i}
$$

Since $\eta$ vanishes on a neighborhood $W$ of $\partial \overline{\mathcal{S}}$ in $\overline{\mathcal{M}}$, the section $\widetilde{s} \equiv \eta s$ of $V$ over $\mathcal{S}$ extends by zero to a continuous section over $\overline{\mathcal{S}}$. Since $\nu$ does not vanish on $\overline{\mathcal{S}}$, we can assume that $\nu$ does not vanish on $\bar{W}$ as well. Furthermore, $\eta$ satisfies the requirements of Lemma 2.22 with $\nu$ replaced by $\left(1-\eta^{\prime}\right) \nu$ and $\delta_{i}^{*}$ replaced by $\delta_{i}^{*} / 2$. Since $\eta$ does not vanish on the complement of $W_{1}$, it follows that $\eta s+t\left(1-\eta^{\prime}\right) \nu$ is transverse to the zero set on $\left(\mathcal{S}-W_{1}\right) \cup \bar{W}$. Thus, for all $t, \epsilon \in \mathbb{R}^{+}$, there exists $\nu^{\prime} \in \Gamma(\overline{\mathcal{S}} ; V)$ such that

$\left.\nu^{\prime}\right|_{\mathcal{S}-W_{1}}=\left.\left(1-\eta^{\prime}\right) \nu\right|_{\mathcal{S}-W_{1}},\left.\quad \nu^{\prime}\right|_{W}=\left.\left(1-\eta^{\prime}\right) \nu\right|_{W}, \quad\left\|\nu^{\prime}-\left(1-\eta^{\prime}\right) \nu\right\|_{C^{2}(\mathcal{S}-W)}<\epsilon$, and $\eta s+t \nu^{\prime}$ is transversal to the zero set on $\mathcal{S}$. Since $\eta s+t \nu^{\prime}$ does not vanish on $\partial \overline{\mathcal{S}}$ and is a positive multiple of $s$ outside of $W_{1}$,

$$
\begin{aligned}
\langle e(V), \overline{\mathcal{S}}\rangle & \left.={ }^{ \pm} \mid\left\{\eta s+t \nu^{\prime}\right\}^{-1}(0)\right\} \mid \\
& ={ }^{ \pm}\left|s^{-1}(0)\right|+\sum_{i \in I_{s}}{ }^{ \pm}\left|\left\{\eta s+t \nu^{\prime}\right\}^{-1}(0) \cap W_{i}\left(\delta_{i}^{*} / 2\right)\right| .
\end{aligned}
$$


On the other hand, by Lemmas $2.20,2.22$,

$$
\sum_{i \in I_{s}}^{ \pm}\left|\left\{\eta s+t \nu^{\prime}\right\}^{-1}(0) \cap W_{i}\left(\delta_{i}^{*} / 2\right)\right|=\sum_{i \in I_{s}^{*}} \operatorname{deg} \rho_{i} \cdot{ }^{ \pm}\left|\psi_{i, \bar{\nu}}^{-1}(0)\right|,
$$

provided $t$ and $\epsilon$ are sufficiently small. Proposition 2.18B follows immediately from equations (2.32) and (2.33), assumptions (A1) and (A3), and the second remark after the statement of the proposition.

\section{Spaces of stable maps}

\subsection{Notation}

In this subsection, we describe our notation for spaces of tuples of stable rational maps and for important vector bundles over them. The zeros of certain sections of these bundles can be identified with rational curves with prescribed singularities. In many cases, using the topological method of Section 2 and the analytic estimates of Subsection 3.2, one can express the number of such zeros in terms of intersection numbers of certain tautological cohomology classes, that are also defined in this subsection. The notation described below is a generalization on that of [17, Subsection 1.3] and [18, Section 2]. Thus, we omit some details.

Definition 3.1 A finite partially ordered set $I$ is a linearly ordered set if for all $i_{1}, i_{2}, h \in I$ such that $i_{1}, i_{2}<h$, either $i_{1} \leq i_{2}$ or $i_{2} \leq i_{1}$.

The term linearly ordered set is sometimes used with a different meaning in combinatorics, as the referee pointed out. We continue to use this term with the meaning of Definition 3.1 to be consistent with earlier papers.

If $I$ is a linearly ordered set, let $\widehat{I}$ be the subset of the non-minimal elements of $I$. For every $h \in \widehat{I}$, denote by $\iota_{h} \in I$ the largest element of $I$ which is smaller than $h$, ie

$$
\iota_{h}=\max \{i \in I: i<h\} .
$$

Definition 3.2 A linearly ordered set $I$ is graded by $I=I^{-} \sqcup I^{+}$if $I-\widehat{I} \subset I^{-}$, $\iota_{h} \in I^{\mp}$ for all $h \in I^{ \pm} \cap \widehat{I}$, and for every $i \in I^{-}$there exists $h \in I^{+}$such that $\iota_{h}=i$. 
A graded linearly ordered set can be represented by an oriented graph. In Figure 2. the small black and large gray dots denote the elements of $I^{-}$and $I^{+}$, respectively. The arrows specify the partial ordering of the linearly ordered set $I$. We use graded linearly ordered sets to encode the structure of an $I^{+}$ tuple of stable maps. The set $I^{-}$will describe the nodes of the domain of the map. For example, the domain of an $I^{+}$-tuple of stable maps with the structure depicted by Figure 2 will have two connected components. One of these components will consist of six irreducible components and contain a double point and two triple points. We give more details below.
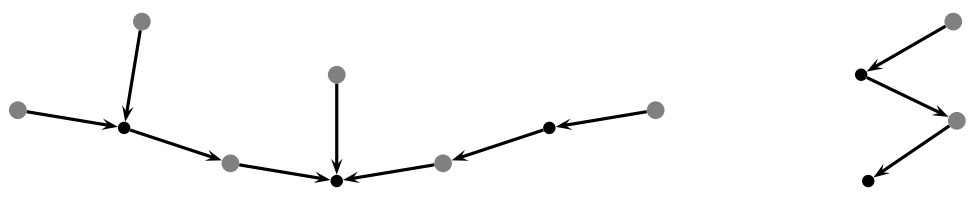

Figure 2: A graded linearly ordered set

If $I=I^{-} \sqcup I^{+}$is a graded linearly ordered set, $I^{-}$and $I^{+}$are linearly ordered sets. We denote by $\widehat{I}^{-}$and $\widehat{I}^{+}$the subsets of the non-minimal elements of $I^{-}$ and $I^{+}$, respectively. If $h \in \widehat{I}^{ \pm}$, we define $\iota_{h}^{ \pm}$as in (3.1), but with $I$ replaced by $\widehat{I}^{ \pm}$. If $h_{1}, h_{2} \in I$, let

$$
\begin{array}{ll}
{\left[h_{1}, h_{2}\right]=\left\{i \in I^{+}: h_{1} \leq i \leq h_{2}\right\},} & {\left[h_{1}, h_{2}\right)=\left\{i \in I^{+}: h_{1} \leq i<h_{2}\right\},} \\
\left(h_{1}, h_{2}\right]=\left\{i \in I^{+}: h_{1}<i \leq h_{2}\right\}, & \left(h_{1}, h_{2}\right)=\left\{i \in I^{+}: h_{1}<i<h_{2}\right\} .
\end{array}
$$

If $I=I^{-} \sqcup I^{+}$is a graded linearly ordered set and $i^{*}$ is an element of $I^{+}$, we define a new graded linearly ordered set

$$
I\left(i^{*}\right)=I^{-}\left(i^{*}\right) \sqcup I^{+}\left(i^{*}\right)
$$

as follows. We take $I^{ \pm}\left(i^{*}\right)=I^{ \pm} \sqcup\left\{i_{ \pm}^{*}\right\}$, where $i_{+}^{*}$ and $i_{-}^{*}$ are new elements. We define a partial ordering $\succ$ on the set $I^{ \pm}\left(i^{*}\right)$ by

$$
\begin{gathered}
h \succ i \text { if } h, i \in I \text { and } h>i ; \quad i \succ i_{ \pm}^{*} \quad \text { if } i \in I \text { and } i>i^{*} ; \\
i_{ \pm}^{*} \succ i \text { if } i \in I \text { and } i^{*} \geq i ; \quad i_{+}^{*} \succ i_{-}^{*} .
\end{gathered}
$$

It is easy to see that $I\left(i^{*}\right)$ is indeed a graded linearly ordered set.

We denote the south pole of the 2 -sphere $S^{2} \subset \mathbb{R}^{3}$ by $\infty$ and identify $S^{2}-\{\infty\}$ with $\mathbb{C}$ via the standard stereographic projection $q_{N}$ mapping the origin in $\mathbb{C}$ to the north pole of $S^{2}$. If $M$ is a finite set, a $\mathbb{P}^{n}$-valued bubble map with 
$M-$ marked points is a tuple $b=(M, I ; x,(j, y), u)$, where $I=I^{-} \sqcup I^{+}$is a graded linearly ordered set, and

$$
\begin{aligned}
x: \widehat{I}^{-} \longrightarrow S^{2}-\{\infty\}, & j: M \longrightarrow I^{+}, \quad y: M \longrightarrow S^{2}-\{\infty\}, \\
\text { and } \quad & u: I^{+} \longrightarrow C^{\infty}\left(S^{2} ; \mathbb{P}^{n}\right)
\end{aligned}
$$

are maps such that

$$
\left(\iota_{h_{1}}, x_{h_{1}}\right) \neq\left(\iota_{h_{2}}, x_{h_{2}}\right), \quad\left(j_{l_{1}}, y_{l_{1}}\right) \neq\left(j_{l_{2}}, y_{l_{2}}\right), \quad\left(\iota_{h}, x_{h}\right) \neq\left(j_{l}, y_{l}\right)
$$

for all $h, h_{1}, h_{2} \in \widehat{I}^{-}$and $l, l_{1}, l_{2} \in M$;

$$
u_{h_{1}}(\infty)=u_{h_{2}}(\infty)
$$

for all $h_{1}, h_{2} \in I^{+}$such that $\iota_{h_{1}}=\iota_{h_{2}}$; and

$$
u_{h}(\infty)=u_{\iota_{h}^{+}}\left(x_{\iota_{h}}\right)
$$

for all $h \in \widehat{I}^{+}$. We associate such a tuple with Riemann surface

$$
\Sigma_{b}=\left(\bigsqcup_{h \in I^{+}} \Sigma_{b, h}\right) / \sim
$$

where $\Sigma_{b, h}=\{h\} \times S^{2}$, and $\left(h_{1}, \infty\right) \sim\left(h_{2}, \infty\right)$ for all $h_{1}, h_{2} \in I^{+}$such that $\iota_{h_{1}}=\iota_{h_{2}}$, and $(h, \infty) \sim\left(\iota_{h}^{+}, x_{\iota_{h}}\right)$ for all $h \in \widehat{I}^{+}$, with marked points $\left(j_{l}, y_{l}\right) \in$ $\Sigma_{b, j_{l}}$, and continuous map $u_{b}: \Sigma_{b} \longrightarrow \mathbb{P}^{n}$, given by $u_{b} \mid \Sigma_{b, h}=u_{h}$ for all $h \in I^{+}$. We require that $\Sigma_{b, h}$ contain at least two singular and/or marked points of $\Sigma_{b}$ other than $(h, \infty)$ if $u_{h *}\left[S^{2}\right]=0 \in H_{2}\left(\mathbb{P}^{n} ; \mathbb{Z}\right)$. In addition, we implicitly consider each point $(h, \infty)$ to be a special marked point. Figure 2 is basically the dual graph of $\Sigma_{b}$. The black dots simply specify which of the special marked points are identified and thus are mapped to the same point in $\mathbb{P}^{n}$. If

$$
b_{1}=\left(M, I_{1} ; x^{(1)},\left(j^{(1)}, y^{(1)}\right), u^{(1)}\right) \quad \text { and } \quad b_{2}=\left(M, I_{2} ; x^{(2)},\left(j^{(2)}, y^{(2)}\right), u^{(2)}\right)
$$

are two bubble maps and $\widetilde{I}$ is a subset of $I_{1}^{+}$and of $I_{2}^{+}$, we say $b_{1}$ and $b_{2}$ are $\widetilde{I}$-equivalent if there exists a homeomorphism $\phi: \Sigma_{b_{1}} \longrightarrow \Sigma_{b_{2}}$ such that $\left.\phi\right|_{\Sigma_{b_{1}, i}}$ is holomorphic for all $i \in I^{+}, \phi\left(\Sigma_{b_{1}, i}\right) \subset \Sigma_{b_{2}, i}$ for all $i \in \widetilde{I}, u_{b_{1}}=u_{b_{2}} \circ \phi$,

$$
\phi\left(j_{l}^{(1)}, y_{l}^{(1)}\right)=\left(j_{l}^{(2)}, y_{l}^{(2)}\right) \quad \text { for all } l \in M,
$$

and for every $i \in I_{1}^{+}$there exists $i^{\prime} \in I_{2}^{+}$such that $\phi(i, \infty)=\left(i^{\prime}, \infty\right)$.

The general structure of bubble maps is described by tuples $\mathcal{T}=(M, I ; j, \underline{d})$, with $d_{i} \in \mathbb{Z}$ specifying the degree of the map $u_{b}$ on $\Sigma_{b, i}$. The above equivalence relation on the set of bubble maps induces an equivalence relation on the set of 
bubble types. If $\widetilde{I} \subset I^{+}$, we denote by $\mathcal{A}(\mathcal{T} \mid \widetilde{I})$ the group of $\widetilde{I}$-automorphisms of $\mathcal{T}$; let $\mathcal{A}(\mathcal{T})=\mathcal{A}(\mathcal{T} \mid \emptyset)$. For each $i \in I^{+}$, let

$$
H_{i} \mathcal{T}=\left\{h \in \widehat{I}^{-}: \iota_{h}=i\right\} \quad \text { and } \quad M_{i} \mathcal{T}=\left\{l \in M: j_{l}=i\right\} .
$$

If $i \in I^{-}$, we put

$$
H_{i} \mathcal{T}=\left\{h \in I^{+}: \iota_{h}=i\right\} \cup \begin{cases}\{i\}, & \text { if } i \in \widehat{I}^{-} \\ \emptyset, & \text { if } i \notin \widehat{I}^{-}\end{cases}
$$

Let $\mathcal{H}_{\mathcal{T}}$ denote the space of all holomorphic bubble maps with structure $\mathcal{T}$. This is a smooth complex manifold; see [10, Chapter 3], for example.

We denote by $\mathcal{U}_{\mathcal{T} \mid \widetilde{I}}$ the set of $\widetilde{I}$-equivalence classes of bubble maps in $\mathcal{H}_{\mathcal{T}}$. Then there exists a smooth submanifold $\mathcal{B}_{\mathcal{T}}$ of $\mathcal{H}_{\mathcal{T}}$ such that $\mathcal{U}_{\mathcal{T} \mid \widetilde{I}}$ is the quotient of $\mathcal{B}_{\mathcal{T}}$ by a natural action of the group

$$
G_{\mathcal{T} \mid \widetilde{I}} \equiv \mathcal{A}(\mathcal{T} \mid \widetilde{I}) \ltimes G_{\mathcal{T}}, \quad \text { where } \quad G_{\mathcal{T}}=\left(S^{1}\right)^{I^{+}} .
$$

For any $i \in \widetilde{I}$, denote by $\mathcal{U}_{\mathcal{T} \mid \widetilde{I}}^{(i)}$ the quotient of $\mathcal{B}_{\mathcal{T}}$ by the group

$$
G_{\mathcal{T} \mid \widetilde{I}}^{(i)} \equiv \mathcal{A}(\mathcal{T} \mid \widetilde{I}) \ltimes G_{\mathcal{T}}^{(i)}, \quad \text { where } \quad G_{\mathcal{T}}^{(i)}=\left(S^{1}\right)^{I^{+}-\{i\}} .
$$

Then, $\mathcal{U}_{\mathcal{T} \mid \widetilde{I}}$ is the quotient of $\mathcal{U}_{\mathcal{T} \mid \widetilde{I}}^{(i)}$ by the residual $S^{1}$-action. If $i \in I^{+}$is fixed by every element of the group $\mathcal{A}(\mathcal{T} \mid \widetilde{I})$, corresponding to the first quotient we obtain a line orbi-bundle $L_{i} \mathcal{T} \longrightarrow \mathcal{U}_{\mathcal{T} \mid \tilde{I}}$. In general, the direct sum of the line bundles $L_{i} \mathcal{T}$ taken over all elements of the orbit $\mathcal{A}(\mathcal{T} \mid \widetilde{I}) \cdot i$ is well-defined. If $h \in \widehat{I}^{+}$, let $\mathcal{F}_{h} \mathcal{T}=L_{\iota_{h}^{+}}^{*} \mathcal{T} \otimes L_{h} \mathcal{T}$.

The Gromov-convergence topology on the space of all holomorphic maps induces a partial ordering on the set of bubble types and their equivalence classes such that the spaces

$$
\overline{\mathcal{U}}_{\widetilde{\mathcal{T}}}^{(i)} \equiv \overline{\mathcal{U}}_{\widetilde{\mathcal{T}} \mid \tilde{I}^{+}}^{(i)}=\bigcup_{\mathcal{T} \leq \widetilde{\mathcal{T}}} \mathcal{U}_{\mathcal{T} \mid \tilde{I}^{+}}^{(i)} \quad \text { and } \quad \overline{\mathcal{U}}_{\tilde{\mathcal{T}}} \equiv \overline{\mathcal{U}}_{\tilde{\mathcal{T}} \mid \tilde{I}^{+}}=\bigcup_{\mathcal{T} \leq \widetilde{\mathcal{T}}} \mathcal{U}_{\mathcal{T} \mid \tilde{I}^{+}}
$$

are compact and Hausdorff. Here $\widetilde{\mathcal{T}} \equiv(M, \widetilde{I} ; \tilde{j}, \underline{\tilde{d}})$, and the unions are disjoint if taken over $\widetilde{I}^{+}$-equivalence classes of bubble types. If $\mathcal{T} \leq \widetilde{\mathcal{T}}$, let

$$
\overline{\mathcal{U}}_{\mathcal{T} \mid \widetilde{\mathcal{T}}}^{(i)}=\overline{\mathcal{U}}_{\mathcal{T} \mid \tilde{I}^{+}}^{(i)} \quad \text { and } \quad \overline{\mathcal{U}}_{\mathcal{T} \mid \widetilde{\mathcal{T}}}=\overline{\mathcal{U}}_{\mathcal{T} \mid \widetilde{I}^{+}} .
$$

The residual $S^{1}$-action on $\mathcal{U}_{\widetilde{\mathcal{T}}}^{(i)}$ extends to an action on $\overline{\mathcal{U}}_{\widetilde{\mathcal{T}}}^{(i)}$, and thus the line orbi-bundle $L_{i} \widetilde{\mathcal{T}} \longrightarrow \mathcal{U}_{\widetilde{\mathcal{T}}}$ extends over $\overline{\mathcal{U}}_{\mathcal{T}}$ as the line bundle $L_{i} \mathcal{T}$. 
For each $l \in M, h \in I^{+}$, and $i \in I^{-}$, we define evaluation maps $\mathcal{H}_{\mathcal{T}} \longrightarrow \mathbb{P}^{n}$ by

$$
\mathrm{ev}_{l}((M, I ; x,(j, y), u))=u_{j_{l}}\left(y_{l}\right), \quad \operatorname{ev}_{h}((M, I ; x,(j, y), u))=u_{h}(\infty),
$$

and $\mathrm{ev}_{i}=\mathrm{ev}_{h}$ if $h \in I^{+}$and $\iota_{h}=i$. These maps descend to all the quotients defined above and induce continuous maps on $\overline{\mathcal{U}}_{\mathcal{T}}$. If $\widetilde{M} \subset M \sqcup I^{-}$and $\mu=\mu_{\widetilde{M}}$ is an $\widetilde{M}$-tuple of submanifolds of $\mathbb{P}^{n}$, let

$$
\mathcal{U}_{\mathcal{T}}(\mu)=\left\{b \in \mathcal{U}_{\mathcal{T}}: \operatorname{ev}_{l}(b) \in \mu_{l} \text { for all } l \in \widetilde{M}\right\},
$$

and define the spaces $\overline{\mathcal{U}}_{\mathcal{T}}(\mu)$ and $\mathcal{U}_{\mathcal{T} \mid \widetilde{\mathcal{T}}}(\mu)$ analogously.

If $\mathcal{T}=(M, I ; j, \underline{d})$ is a bubble type and $k \in I^{+}$, we define the bubble type $\mathcal{T}_{k} \equiv\left(M_{k}, I_{k} ; j^{(k)}, \underline{d}^{(k)}\right)$ by

$$
M_{k}=M_{k} \mathcal{T} \sqcup H_{k} \mathcal{T} ; \quad I_{k}=\left\{k, \iota_{k}\right\} \subset I ; \quad j_{l}^{(k)}=k \quad \text { for all } l \in M_{k} ; \quad d_{k}^{(k)}=d_{k} .
$$

Let $\mathcal{U}_{\mathcal{T}, \mathcal{T}}=\prod_{k \in I^{+}} \mathcal{U}_{\mathcal{T}_{k}}$ and $\overline{\mathcal{U}}_{\mathcal{T}, \mathcal{T}}=\prod_{k \in I^{+}} \overline{\mathcal{U}}_{\mathcal{T}_{k}}$. Note that the spaces $\mathcal{U}_{\mathcal{T}}$ and $\overline{\mathcal{U}}_{\mathcal{T}}$ are contained in $\mathcal{U}_{\mathcal{T}, \mathcal{T}}$ and $\overline{\mathcal{U}}_{\mathcal{T}, \mathcal{T}}$, respectively.

Suppose $\mathcal{T}=(M, I ; j, \underline{d})$ is a bubble type, $i^{*}$ is an element of $I^{+}$such that $d_{i^{*}} \neq 0$ and $M_{0}$ is nonempty subset of $M_{i^{*}} \mathcal{T}$. We define bubble type

$$
\mathcal{T}\left(M_{0}\right) \equiv\left(M, I\left(i^{*}\right) ; j^{\prime}, \underline{d}^{\prime}\right)
$$

by

$$
j_{l}^{\prime}= \begin{cases}i^{*}, & \text { if } l \in M_{0} ; \\
i_{+}^{*}, & \text { if } l \in M_{i^{*}} \mathcal{T}-M_{0} ; \quad d_{i}^{\prime}=\left\{\begin{array}{ll}
0, & \text { if } i=i^{*} \\
j_{l}, & \text { otherwise }
\end{array} d_{i^{*},} \text { if } i=i_{+}^{*}\right. \\
d_{i}, & \text { otherwise }\end{cases}
$$

If $l \in M$, we will write $\mathcal{T}(l)$ for $\mathcal{T}(\{l\})$. In Figure 3, we show the domain of an element of the space $\mathcal{U}_{\mathcal{T}}$, where $I=\left\{i^{*}\right\}$ is a single-element set, and the domain of an element of the space $\mathcal{U}_{\mathcal{T}\left(M_{0}\right)}$, where $M_{0}=\left\{l_{1}, l_{2}\right\}$ is a two-element set.

In Figure 3, as well as in later figures, we denote each component of the domain by a disk and shade the component(s) on which the map into $\mathbb{P}^{n}$ is nonconstant. We indicate marked points on the ghost components, ie the components on which the map is constant, by putting small dots on the boundary of the corresponding disk. The point labeled by $i^{*}$, ie the same way as the component, is the special marked point $\left(i^{*}, \infty\right)$. Lemma 3.4 and Proposition 3.5. as well as the decomposition (3.4), show that it is crucial to clearly distinguish between ghost and non-ghost components. 


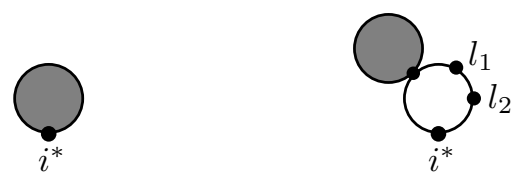

Figure 3: The domains of elements of $\mathcal{U}_{\mathcal{T}}$ and $\mathcal{U}_{\mathcal{T}\left(M_{0}\right)}$

If $[N] \equiv\{1, \ldots, N\}$ is a subset of $M$ such that the set $M-[N]$ contains no positive integers, we put

$$
c_{1}\left(\mathcal{L}_{i^{*}}^{*} \mathcal{T}\right) \equiv c_{1}\left(L_{i^{*}}^{*} \mathcal{T}\right)-\sum_{\emptyset \neq M_{0} \subset M_{i^{*}} \mathcal{T} \cap[N]} P D_{\overline{\mathcal{U}}_{\mathcal{T}, \mathcal{T}}}\left[\overline{\mathcal{U}}_{\mathcal{T}\left(M_{0}\right), \mathcal{T}\left(M_{0}\right)}\right] \in H^{2}\left(\overline{\mathcal{U}}_{\mathcal{T}, \mathcal{T}}\right) .
$$

By Proposition 3.5. $\overline{\mathcal{U}}_{\mathcal{T}, \mathcal{T}}$ is an ms-orbifold, while $\overline{\mathcal{U}}_{\mathcal{T}\left(M_{0}\right), \mathcal{T}\left(M_{0}\right)}$ is an mssuborbifold of $\overline{\mathcal{U}}_{\mathcal{T}, \mathcal{T}}$. Thus, the cohomology class on the left-hand side of (3.2) is well-defined. We illustrate definition (3.2) in Figure 4 in the case $I^{+}=\left\{i^{*}\right\}$ is a single-element set. In this figure, as well in the future ones, we denote spaces of tuples of stable maps by drawing a picture of the domain of a typical element of such a space. On the other hand, let

$$
\pi_{i}: \overline{\mathcal{U}}_{\mathcal{T}, \mathcal{T}} \longrightarrow \overline{\mathcal{U}}_{\mathcal{T}_{i}^{\prime}}, \quad \text { where } \quad \mathcal{T}_{i}^{\prime}=\left(M_{i}-[N], I_{i} ; j^{(i)}, \underline{d}^{(i)}\right),
$$

be the composition of the projection onto the $i$ th factor with the appropriate forgetful map. By [11, Lemma 2.2.2],

$$
c_{1}\left(\mathcal{L}_{i^{*}}^{*} \mathcal{T}\right)=\pi_{i^{*}}^{*} \psi_{i^{*}}
$$

where $\psi_{i^{*}}$ is the first chern class of the universal cotangent line bundle at the marked point $\left(i^{*}, \infty\right)$ over the moduli space

$$
\overline{\mathfrak{M}}_{0,\left(M_{i}-[N]\right) \sqcup\left\{i^{*}\right\}}\left(d_{i^{*}}, \mathbb{P}^{n}\right)
$$

of stable rational degree- $d_{i^{*}}$ maps into $\mathbb{P}^{n}$ with marked points labeled by the set $\left(M_{i}-[N]\right) \sqcup\left(i^{*}, \infty\right)$. In particular, $c_{1}\left(\mathcal{L}_{i^{*}}^{*} \mathcal{T}\right)$ is the first chern class of a line orbi-bundle over $\overline{\mathcal{U}}_{\mathcal{T}, \mathcal{T}}$. Whenever the bubble type $\mathcal{T}$ is clear from context, we will write $c_{1}\left(L_{i}^{*}\right)$ and $c_{1}\left(\mathcal{L}_{i}^{*}\right)$ for $c_{1}\left(L_{i}^{*} \mathcal{T}\right)$ and $c_{1}\left(\mathcal{L}_{i}^{*} \mathcal{T}\right)$, respectively. If $\widetilde{M}$ is a subset of $M \sqcup I^{-}$that contains $[N]$ and $\mu$ is an $\widetilde{M}$-tuple of constraints in $\mathbb{P}^{n}$ such that $\mu_{l_{1}} \cap \mu_{l_{2}}=\emptyset$ for all distinct elements $l_{1}, l_{2}$ of $N$,

$$
\begin{aligned}
{\left[\overline{\mathcal{U}}_{\mathcal{T}(l)}(\mu)\right] \cap c_{1}\left(\mathcal{L}_{i^{*}}^{*} \mathcal{T}\right) } & =\left[\overline{\mathcal{U}}_{\mathcal{T}(l)}(\mu)\right] \cap c_{1}\left(L_{i_{+}^{*}}^{*} \mathcal{T}(l)\right) \\
& \left.=\overline{\mathcal{U}}_{\mathcal{T}(l)}(\mu)\right] \cap c_{1}\left(\mathcal{L}_{i_{+}^{*}}^{*} \mathcal{T}(l)\right) \text { for all } l \in M_{i^{*}} \mathcal{T} .
\end{aligned}
$$

Note that by Lemma 3.4. $\mathcal{U}_{\mathcal{T}(l)}(\mu)$ is a pseudocycle in $\overline{\mathcal{U}}_{\mathcal{T}, \mathcal{T}}$ and thus induces a homology class. The first equality in (3.3) can be deduced from [11, Subsection 3.2]. 


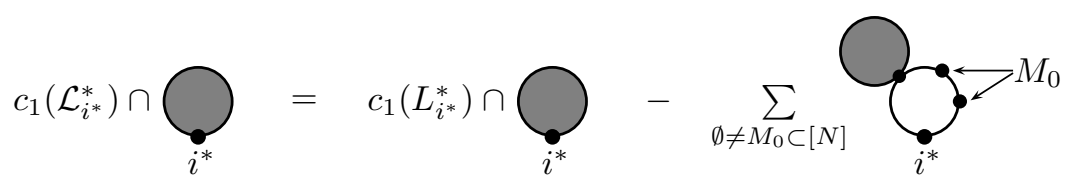

Figure 4: An example of Definition (3.2)

We are now ready to formally explain the notation involved in the statement of Theorem 1.1. Let $n, d, N$ be positive integers and let $\mu$ be an $N$-tuple of constraints in $\mathbb{P}^{n}$. If $k \geq 1$, denote by $\overline{\mathcal{V}}_{k}(\mu)$ the quotient of the disjoint union of the spaces $\overline{\mathcal{U}}_{\mathcal{T}}(\mu)$ taken over all bubble types $\mathcal{T}=\left([N], I_{k} ; j, \underline{d}\right)$ such that

$$
I_{k}^{+}=\{\widetilde{1}, \ldots, \widetilde{k}\}, \quad \sum d_{i}=d
$$

and $I_{k}^{-}=\{\widehat{0}\}$ is a one-element set, by the natural action of the symmetric group $S_{k}$. We define the spaces $\mathcal{V}_{k}(\mu)$ similarly. Denote by

$$
\eta_{\widehat{0}, l}, \widetilde{\eta}_{\widehat{0}, l} \in H^{2 l}\left(\overline{\mathcal{V}}_{k}(\mu)\right)
$$

the cohomology classes such that $\pi^{*} \eta_{\widehat{0}, l}$ and $\pi^{*} \widetilde{\eta}_{\widehat{0}, l}$ are the sum of all degree- $l$ monomials in

$$
\left\{c_{1}\left(\mathcal{L}_{\widetilde{1}}^{*}\right), \ldots, c_{1}\left(\mathcal{L}_{\widetilde{k}}^{*}\right)\right\} \quad \text { and } \quad\left\{c_{1}\left(L_{\widetilde{1}}^{*}\right), \ldots, c_{1}\left(L_{\widetilde{k}}^{*}\right)\right\},
$$

respectively, where $\pi: \bigcup_{\mathcal{T}} \overline{\mathcal{U}}_{\mathcal{T}} \longrightarrow \overline{\mathcal{V}}_{k}(\mu)$ is the quotient projection map. For example, if $k=2$,

$$
\pi^{*} \eta_{\widehat{0}, 3}=c_{1}^{3}\left(\mathcal{L}_{\widetilde{1}}^{*}\right)+c_{1}^{3}\left(\mathcal{L}_{\widetilde{2}}^{*}\right)+c_{1}^{2}\left(\mathcal{L}_{\widetilde{1}}^{*}\right) c_{1}\left(\mathcal{L}_{\widetilde{2}}^{*}\right)+c_{1}\left(\mathcal{L}_{\widetilde{1}}^{*}\right) c_{1}^{2}\left(\mathcal{L}_{\widetilde{2}}^{*}\right) .
$$

Let $a_{\widehat{0}}=\operatorname{ev}_{\widehat{0}}^{*} c_{1}\left(\gamma_{\mathbb{P}^{n}}^{*}\right) \in H^{2}\left(\overline{\mathcal{V}}_{k}(\mu)\right)$, where $\gamma_{\mathbb{P}^{n}} \longrightarrow \mathbb{P}^{n}$ is the tautological line bundle.

Suppose $\widetilde{\mathcal{T}}=(M, \widetilde{I} ; \tilde{j}, \underline{\tilde{d}})$ and $\mathcal{T}=(M, I ; j, \underline{d})$ are bubble types, such that $\mathcal{T}<\widetilde{\mathcal{T}}, \widetilde{M}$ is a subset of $M \sqcup \widetilde{I}^{-}$, and $\mu$ is an $\widetilde{M}$-tuple of constraints in $\mathbb{P}^{n}$. Let

$$
I_{0}=\left\{i \in I^{+}: d_{i}=0\right\} .
$$

Suppose $I_{0} \subset I^{+}-\widehat{I}^{+}$and for every $i \in I_{0}$ there exists $h \in I^{+}$such that $i<h$. We can then construct a decomposition of the spaces $\mathcal{U}_{\mathcal{T} \mid \widetilde{\mathcal{T}}}(\mu)$ and $\overline{\mathcal{U}}_{\mathcal{T} \mid \widetilde{\mathcal{T}}}(\mu)$ which is useful in computations as follows. Let $\overline{\mathcal{T}}=(\bar{M}, \bar{I} ; \bar{j}, \bar{d})$ be the bubble type given by

$$
\bar{M}=M-\bigcup_{i \in I_{0}} M_{i} \mathcal{T} \quad \text { and } \quad \bar{I}=\left(I \sqcup \bigcup_{i \in I_{0}} M_{i} \mathcal{T}\right) / \sim
$$


where $\iota_{i} \sim h$ if $i \in I_{0}$ and $h \in\left(\{i\} \cup H_{i} \mathcal{T}\right) \sqcup M_{i} \mathcal{T}$. The set $\bar{I}$ is a graded linearly ordered set, with its structure induced from $I$. Let $\bar{d}_{i}=d_{i}$ and $\bar{j}_{l}=j_{l}$ whenever $i \in \bar{I}^{+} \subset I^{+}$and $l \in \bar{M} \subset M$. Let $\widetilde{M^{\prime}}$ be the image of $\widetilde{M}$ under the quotient projection map $I \sqcup M \longrightarrow \bar{I} \sqcup \bar{M}$. We identify the $\widetilde{M}$-tuple $\mu$ of constraints with an $\widetilde{M}^{\prime}$-tuple $\bar{\mu}$ of constraints in $\mathbb{P}^{n}$ by

$$
\bar{\mu}_{\bar{l}}=\bigcap_{[l]=\bar{l}} \mu_{l} .
$$

Since every degree-zero holomorphic map is constant, we obtain

$$
\begin{aligned}
\mathcal{U}_{\mathcal{T} \mid \tilde{\mathcal{T}}}(\mu) & \approx\left(\prod_{i \in I_{0}} \mathfrak{M}_{\left(i \cup H_{i} \mathcal{T}\right) \sqcup M_{i} \mathcal{T}} \times \mathcal{U}_{\overline{\mathcal{T}}}(\mu)\right) / \mathcal{A}\left(\mathcal{T} \mid \widetilde{I}^{+}\right) \\
& \subset\left(\prod_{i \in I_{0}} \overline{\mathfrak{M}}_{\left(i \cup H_{i} \mathcal{T}\right) \sqcup M_{i} \mathcal{T}} \times \overline{\mathcal{U}}_{\overline{\mathcal{T}}}(\mu)\right) / \mathcal{A}\left(\mathcal{T} \mid \widetilde{I}^{+}\right) .
\end{aligned}
$$

Here $\overline{\mathfrak{M}}_{\left(i \cup H_{i} \mathcal{T}\right) \sqcup M_{i} \mathcal{T}}$ denotes the Deligne-Mumford moduli space of rational curves with marked points labeled by the set $\left(i \cup H_{i} \mathcal{T}\right) \sqcup M_{i} \mathcal{T}$, and also $\mathfrak{M}_{\left(i \cup H_{i} \mathcal{T}\right) \sqcup M_{i} \mathcal{T}}$ denotes the main stratum of $\overline{\mathfrak{M}}_{\left(i \cup H_{i} \mathcal{T}\right) \sqcup M_{i} \mathcal{T}}$. If $i \in I_{0} \subset \widetilde{I}^{+}$, then by definition the line bundle $L_{i} \widetilde{\mathcal{T}}$ restricts to the universal tangent line bundle at the marked point $i$ over $\overline{\mathfrak{M}}_{\left(i \cup H_{i} \mathcal{T}\right) \sqcup M_{i} \mathcal{T}}$. We will denote this bundle by $\gamma_{\mathcal{T} ; i}$. If $\widetilde{d}_{i} \neq 0$ for all $i \in \widetilde{I}^{+}$and $M_{0} \subset[N] \cap M_{i} \mathcal{T}$ for some $i \in \widetilde{I}^{+}$, we will write $\tilde{\mathcal{T}} / M_{0}$ for the bubble type $\overline{\mathcal{T}}$ corresponding to $\mathcal{T}=\tilde{\mathcal{T}}\left(M_{0}\right)$ under the construction of this paragraph. The decomposition (3.4) for the bubble $\mathcal{T}\left(M_{0}\right)$ of Figure 3 is illustrated in Figure 5

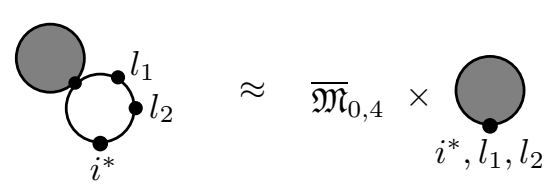

Figure 5: An example of the decomposition (3.4)

\subsection{Structural descriptions}

In this subsection, we define certain bundle sections over the spaces $\mathcal{U}_{\mathcal{T} \mid \tilde{\mathcal{T}}}$. These sections are central to this paper, as the zeros of these and closely related sections count rational curves with pre-specified singularities. We state a basic transversality lemma that implies that these sections are well-behaved over $\mathcal{U}_{\mathcal{T} \mid \widetilde{\mathcal{T}}}$ 
in most cases. The general structure of the spaces $\overline{\mathcal{U}}_{\widetilde{\mathcal{T}}}(\mu)$ and the behavior of the sections near the boundary strata are described by Proposition 3.5

Let $q_{S}$ denote the standard stereographic projection $\mathbb{C} \longrightarrow S^{2}$ mapping the origin in $\mathbb{C}$ to the south pole of $S^{2}$. Suppose

$$
b=(M, I ; x,(j, y), u) \in \mathcal{B}_{\mathcal{T}}
$$

and $m \in \mathbb{Z}^{+}$. If $i \in I^{+}$, let

$$
\mathcal{D}_{\mathcal{T}, i}^{(m)} b=\left.\frac{1}{m !} \frac{D^{m-1}}{d s^{m-1}} \frac{d}{d s}\left(u_{i} \circ q_{S}\right)\right|_{(s, t)=0},
$$

where $(s, t)$ are the real and imaginary coordinates on $\mathbb{C}$ and $\frac{D^{m-1}}{d s^{m-1}}$ denotes the $(m-1)$ st covariant derivative with respect to the Levi-Civita connection of some metric $g_{b, i}$ on $\mathbb{P}^{n}$. If $h \in \widehat{I}^{-}$and $l \in M$, we similarly define

$\mathcal{D}_{\mathcal{T}, h}^{(m)} b=\left.\frac{1}{m !} \frac{D^{m-1}}{d s^{m-1}} \frac{d}{d s} u_{\iota_{h}}\left(x_{h}+s\right)\right|_{s=0} \quad$ and $\quad \mathcal{D}_{\mathcal{T}, l}^{(m)} b=\left.\frac{1}{m !} \frac{D^{m-1}}{d s^{m-1}} \frac{d}{d s} u_{j_{l}}\left(y_{l}+s\right)\right|_{s=0}$ Here we take covariant derivatives with respect to some metrics $g_{b, h}$ and $g_{b, l}$

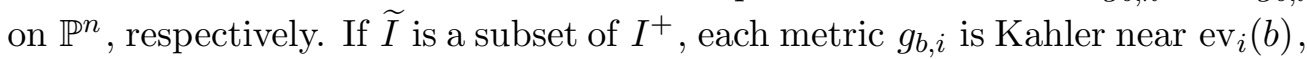
and the family $\left\{g_{b, i}\right\}$ is invariant under the action of the group of $G_{\mathcal{T} \mid \widetilde{I}}, \mathcal{D}_{\mathcal{\mathcal { T }}, i}^{(m)}$ induces a section of $\operatorname{Hom}\left(L_{i}^{\otimes m} \mathcal{T}, \mathrm{ev}_{i}^{*} T \mathbb{P}^{n}\right)$ over $\mathcal{U}_{\mathcal{T} \mid \widetilde{I}}$ given by

$$
\mathcal{D}_{\mathcal{T}, i}^{(m)}\left[b, c_{i}\right]=c_{i} \mathcal{D}_{\mathcal{T}, i}^{(m)} b, \quad \text { if } b \in \mathcal{B}_{\mathcal{T}}, c_{i} \in \mathbb{C} .
$$

Under analogous circumstances, $\mathcal{D}_{\mathcal{T}, h}^{(m)}$ and $\mathcal{D}_{\mathcal{T}, l}^{(m)}$ induce sections of

$$
\operatorname{Hom}\left(L_{\iota_{h}}^{* \otimes m} \mathcal{T}, \mathrm{ev}_{h}^{*} T \mathbb{P}^{n}\right) \quad \text { and } \quad \operatorname{Hom}\left(L_{j_{l}}^{* \otimes m} \mathcal{T}, \mathrm{ev}_{l}^{*} T \mathbb{P}^{n}\right),
$$

respectively. In a certain sense, the choice of the metrics does not matter, since $\mathcal{D}_{\mathcal{T}, i}^{(m)} b, \mathcal{D}_{\mathcal{T}, h}^{(m)} b$, and $\mathcal{D}_{\mathcal{T}, l}^{(m)} b$ are well-defined modulo the image of the lower-order derivatives, and only these quotients have a geometric meaning. However, the method of [17. Subsection 2.5] for proving the explicit estimates of Proposition 3.5 makes use of special properties of the metric near the point where the derivatives are taken. Thus, we put

$$
g_{b, i}=g_{\mathbb{P}^{n}, \mathrm{ev}_{i}(b)}, \quad g_{b, h}=g_{\mathbb{P}^{n}, \mathrm{ev}_{h}(b)}, \quad \text { and } \quad g_{b, l}=g_{\mathbb{P}^{n}, \mathrm{ev}_{l}(b)},
$$

where $g_{\mathbb{P}^{n}}$, is as in Lemma 3.3, which is exactly [17, Lemma 2.1].

Lemma 3.3 There exist $r_{\mathbb{P}^{n}}>0$ and a smooth family of Kahler metrics

$$
\left\{g_{\mathbb{P}^{n}, q}: q \in \mathbb{P}^{n}\right\}
$$

on $\mathbb{P}^{n}$ with the following property. If $B_{q}\left(q^{\prime}, r\right) \subset \mathbb{P}^{n}$ denotes the $g_{\mathbb{P}^{n}, q}$-geodesic ball about $q^{\prime}$, the triple $\left(B_{q}\left(q, r_{\mathbb{P}^{n}}\right), J, g_{\mathbb{P}^{n}, q}\right)$ is isomorphic to a ball in $\mathbb{C}^{n}$ for all $q \in \mathbb{P}^{n}$. 
Suppose $\widetilde{\mathcal{T}}=(M, \widetilde{I} ; \widetilde{j}, \underline{\widetilde{d}})$ and $\mathcal{T} \equiv(M, I ; j, \underline{d})$ are bubble types such that $\mathcal{T}<\widetilde{\mathcal{T}}$. For each $k \in \widetilde{I}^{+}$, let $\widetilde{\mathcal{T}}_{k} \equiv\left(M_{k}, \widetilde{I}_{k} ; \widetilde{j}^{(k)}, \widetilde{\widetilde{d}}^{(k)}\right)$ be the one-component bubble type defined as in Subsection 3.1 and let

$$
\mathcal{T}_{k}(\widetilde{\mathcal{T}}) \equiv\left(M_{k}, I_{k} ; j^{(k)}, \underline{d}^{(k)}\right)<\widetilde{\mathcal{T}}_{k}
$$

be the bubble type given by

$$
\begin{gathered}
I_{k}=\left\{\iota_{k}\right\} \cup\left\{h \in I: h \geq k ; h \nsupseteq h^{\prime} \text { for all } h^{\prime} \in \widetilde{I} \text { such that } h^{\prime}>k\right\}, \\
j_{l}^{(k)}=j_{l} \text { for all } l \in M_{k} ; \quad d_{h}^{(k)}=d_{h} \text { for all } h \in I_{k}^{+} .
\end{gathered}
$$

Let $\mathcal{U}_{\widetilde{\mathcal{T}}, \mathcal{T}}=\prod_{k \in \widetilde{I}^{+}} \mathcal{U}_{\mathcal{T}_{k}(\widetilde{\mathcal{T}}) \mid \widetilde{\mathcal{T}}_{k}} \subset \overline{\mathcal{U}}_{\widetilde{\mathcal{T}}, \widetilde{\mathcal{T}}}$. If $\widetilde{M}$ is a subset of $\widetilde{I}^{-} \cup M$, we define an evaluation map

$$
\begin{gathered}
\operatorname{ev}_{\widetilde{\mathcal{T}}, \widetilde{M}}: \overline{\mathcal{U}}_{\widetilde{\mathcal{T}}, \widetilde{\mathcal{T}}} \equiv \prod_{k \in \widetilde{I}^{+}} \overline{\mathcal{U}}_{\mathcal{T}_{k}} \longrightarrow \mathcal{X}_{\widetilde{\mathcal{T}}}(\widetilde{M}) \equiv\left(\mathbb{P}^{n}\right)^{\widehat{\widetilde{I}}^{-}} \sqcup \widetilde{I}^{+} \sqcup(\widetilde{M} \cap M) \\
\text { by } \quad \pi_{l}\left(\operatorname{ev}_{\widetilde{\mathcal{T}}, \widetilde{M}}(b)\right)= \begin{cases}\operatorname{ev}_{l}\left(b_{k}\right), & \text { if } l \in M_{k} ; \\
\operatorname{ev}_{l}\left(b_{l}\right), & \text { if } l \in \widetilde{I}^{+} ; \\
\operatorname{ev}_{l}\left(b_{\iota l}\right), & \text { if } l \in \widetilde{\widetilde{I}}^{-} .\end{cases}
\end{gathered}
$$

If $\mu$ is an $\widetilde{M}$-tuple of submanifolds in $\mathbb{P}^{n}$, let

$$
\begin{aligned}
\Delta_{\widetilde{\mathcal{T}}}(\mu)=\prod_{i \in \widetilde{I}^{-}}\left\{\left(x_{h}\right)_{h \in H_{i} \widetilde{\mathcal{T}}} \in\left(\mathbb{P}^{n}\right)^{H_{i} \widetilde{\mathcal{T}}}: \begin{array}{l}
x_{h}=x_{l} \text { for all } h, l \in H_{i} \widetilde{\mathcal{T}}, \\
x_{h} \in \mu_{i} \text { if } i \in \widetilde{M}
\end{array}\right\} \\
\times \prod_{l \in(\widetilde{M} \cap M)} \mu_{l} \subset \mathcal{X}_{\widetilde{\mathcal{T}}}(\widetilde{M}) .
\end{aligned}
$$

We denote by $\left.\mathcal{N} \Delta_{\widetilde{\mathcal{T}}}(\mu) \subset T \mathcal{X}_{\widetilde{\mathcal{T}}}(\widetilde{M})\right|_{\Delta_{\tilde{\mathcal{T}}}(\mu)}$ the normal bundle of $\Delta_{\widetilde{\mathcal{T}}}(\mu)$ in $\mathcal{X}_{\widetilde{\mathcal{T}}}(\widetilde{M})$ as well as an extension of this normal bundle to a neighborhood of $\Delta_{\widetilde{\mathcal{T}}}(\mu)$ in $\mathcal{X}_{\widetilde{\mathcal{T}}}(\widetilde{M})$. By definition,

$$
\mathcal{U}_{\widetilde{\mathcal{T}}}(\mu)=\operatorname{ev}_{\tilde{\mathcal{T}}, \widetilde{M}}^{-1}\left(\Delta_{\tilde{\mathcal{T}}}(\mu)\right) \cap \mathcal{U}_{\tilde{\mathcal{T}}, \widetilde{\mathcal{T}}} \quad \text { and } \quad \mathcal{U}_{\mathcal{T} \mid \widetilde{\mathcal{T}}}(\mu)=\operatorname{ev}_{\widetilde{\mathcal{T}}, \widetilde{M}}^{-1}\left(\Delta_{\widetilde{\mathcal{T}}}(\mu)\right) \cap \mathcal{U}_{\widetilde{\mathcal{T}}, \mathcal{T}} .
$$

The following lemma and now-standard arguments, such as in [10, Chapter 3], imply that $\mathcal{U}_{\widetilde{\mathcal{T}}, \mathcal{T}}(\mu)$ is a smooth orbifold, if $\mu$ is a tuple of constraints in general position:

Lemma 3.4 Suppose $u: S^{2} \longrightarrow \mathbb{P}^{n}$ is a holomorphic map of degree $d$, $z_{1}, \ldots, z_{k} \in S^{2}, \quad v_{i} \in T_{z_{i}} S^{2}-\{0\}$ for $i=1, \ldots, k, \quad$ and $\quad m_{1}, \ldots, m_{k} \in \mathbb{Z}^{+}$. 
If $d+1 \geq \sum_{i} m_{i}$, the map

$$
\phi:\left\{\xi \in \Gamma\left(S^{2} ; u^{*} T \mathbb{P}^{n}\right): \bar{\partial} \xi=0\right\} \longrightarrow \bigoplus_{i=1}^{i=k} \bigoplus_{j=1}^{j=m_{i}} T_{u\left(z_{i}\right)} \mathbb{P}^{n}, \quad \phi_{i, j}(\xi)=\left.D_{v_{i}}^{j-1} \xi\right|_{z_{i}},
$$

is surjective.

In the statement of this lemma, $\left.D_{v_{i}}^{j} \xi\right|_{z_{i}}$ denotes the $j$ th covariant derivative of $\xi$ along $u$ in the direction of $v$. The meaning of the lemma is the same no matter what connection is used near each point. The proof is a very slight generalization of that of [17, Corollary 6.3]. Lemma 3.4 implies that if $d$ is sufficiently high, a certain section over the space of degree- $d$ holomorphic maps is transverse to the zero set. In many actual computations, low, but positivedegree, cases will not appear for simple geometric reasons. For example, the space of degree-one maps whose image is a cuspidal curve is empty. Thus, if $k=1$ and $m_{1}=2$, the relevant implication of the lemma is valid as long as $d>0$.

Let

$$
\mathcal{F}_{\widetilde{\mathcal{T}}} \mathcal{T}=\bigoplus_{h \in I^{+}-\tilde{I}} \mathcal{F}_{h} \mathcal{T}=\bigoplus_{k \in \tilde{I}^{+}} \mathcal{F}\left\{\mathcal{T}_{k}(\widetilde{\mathcal{T}})\right\} \longrightarrow \mathcal{U}_{\widetilde{\mathcal{T}}, \mathcal{T}}
$$

If $\tilde{\mathcal{T}}$ is a bubble type such that $\widehat{\widetilde{I}}^{-}=\emptyset$, we will write $\mathcal{F} \mathcal{T}$ for $\mathcal{F}_{\tilde{\mathcal{T}}} \mathcal{T}$. By (2) of Proposition [3.5] $\mathcal{F}_{\widetilde{\mathcal{T}}} \mathcal{T}$ is the "normal bundle" of $\mathcal{U}_{\widetilde{\mathcal{T}}, \mathcal{T}}$ in $\overline{\mathcal{U}}_{\tilde{\mathcal{T}}, \tilde{\mathcal{T}}}$. Part (3) of Proposition 3.5] describes the behavior of various evaluation maps and bundle sections over $\mathcal{U}_{\widetilde{\mathcal{T}}, \widetilde{\mathcal{T}}}$ near the stratum $\mathcal{U}_{\widetilde{\mathcal{T}}, \mathcal{T}}$ of the boundary of $\overline{\mathcal{U}}_{\widetilde{\mathcal{T}}, \widetilde{\mathcal{T}}}$. However, before we can state the relevant expansions, we need to introduce more notation.

If $k \in \widetilde{I}^{+}, h_{1}, h_{2} \in I_{k}^{+}$, and $l \in M_{k}$, let

$$
\begin{gathered}
i_{\mathcal{T}}\left(h_{1}, h_{2}\right)=\max \left\{i \in I_{k}^{+}: i \leq h_{1}, i \leq h_{2}\right\}, \quad i_{\mathcal{T}}\left(l, h_{2}\right)=i_{\mathcal{T}}\left(j_{l}, h_{2}\right) ; \\
\chi_{\mathcal{T}, h_{1}}\left(h_{2}\right)= \begin{cases}0, & \text { if } d_{i}=0 \text { for all } i \in\left[i_{\mathcal{T}}\left(h_{1}, h_{2}\right), h_{1}\right] \cup\left[i_{\mathcal{T}}\left(h_{1}, h_{2}\right), h_{2}\right] ; \\
1, & \text { if } d_{i}=0 \text { for all } i \in\left[i_{\mathcal{T}}\left(h_{1}, h_{2}\right), h_{1}\right] \cup\left[i_{\mathcal{T}}\left(h_{1}, h_{2}\right), h_{2}\right]-\left\{h_{2}\right\}, \\
2, & \text { but } d_{h_{2}} \neq 0 ;\end{cases}
\end{gathered}
$$

Put $\chi_{h_{1}}(\mathcal{T})=\left\{h_{2} \in I_{k}^{+}: \chi_{\mathcal{T}, h_{1}}\left(h_{2}\right)=1\right\}$ and $\chi_{l}(\mathcal{T})=\left\{h_{2} \in \chi_{j_{l}}(\mathcal{T}): h_{2} \not \leq j_{l}\right\}$. If

$$
v=\left[(M, I ; x,(j, y), u),\left(v_{h}\right)_{h \in I^{+}-\tilde{I}}\right] \in \mathcal{F}_{\tilde{\mathcal{T}}} \mathcal{T}=\bigoplus_{k \in I^{+}-\tilde{I}} \mathcal{F}_{k} \mathcal{T}
$$


and $m, m^{\prime} \in \mathbb{Z}$, let

$$
\begin{aligned}
& x_{h_{1} ; h_{2}}(v)=\sum_{h \in\left(i_{\mathcal{T}}\left(h_{1}, h_{2}\right), h_{2}\right]} x_{\iota_{h}} \prod_{i \in\left(i_{\mathcal{T}}\left(h_{1}, h_{2}\right), h\right)} v_{i} \in L_{i_{\mathcal{T}}\left(h_{1}, h_{2}\right)}^{*} \mathcal{T} ; \\
& y_{h_{1} ; l}(v)=\sum_{h \in\left(i_{\mathcal{T}}\left(h_{1}, l\right), j_{l}\right]} x_{\iota_{h}} \prod_{i \in\left(i_{\mathcal{T}}\left(h_{1}, l\right), h\right)} v_{i}+y_{l} \prod_{i \in\left(i_{\mathcal{T}}\left(h_{1}, l\right), j_{l}\right]} v_{i} \in L_{i_{\mathcal{T}}\left(h_{1}, l\right)}^{*} \mathcal{T} . \\
& \rho_{\mathcal{T}, h_{1} ; h_{2}}^{\left(m ; m^{\prime}\right)}(v)=\left(\bigotimes_{i \in\left(k, i_{\mathcal{T}}\left(h_{1}, h_{2}\right)\right]} v_{i}\right)^{\otimes(-m)} \otimes\left(\bigotimes_{i \in\left(i_{\mathcal{T}}\left(h_{1}, h_{2}\right), h_{2}\right]} v_{i}\right)^{\otimes m^{\prime}} \in \widetilde{\mathcal{F}}_{h_{1} ; h_{2}}^{\left(m ; m^{\prime}\right)} \mathcal{T}, \\
& \text { where } \quad \widetilde{\mathcal{F}}_{h_{1} ; h_{2}}^{\left(m ; m^{\prime}\right)} \mathcal{T}=\left(\bigotimes_{i \in\left(k, i_{\mathcal{T}}\left(h_{1}, h_{2}\right)\right]} \mathcal{F}_{i} \mathcal{T}\right)^{\otimes(-m)} \otimes\left(\bigotimes_{i \in\left(i_{\mathcal{T}}\left(h_{1}, h_{2}\right), h_{2}\right]} \mathcal{F}_{i} \mathcal{T}\right)^{\otimes m^{\prime}} .
\end{aligned}
$$

The map $\rho_{\mathcal{T}, h_{1} ; h_{2}}^{\left(m ; m^{\prime}\right)}$ is defined if $h_{1}=k$ or $v \notin Y\left(\mathcal{F}_{\widetilde{\mathcal{T}}} \mathcal{T} ; I^{+}-\widetilde{I}\right)$. If $i \in I_{k}^{-}-\widetilde{I}$, we define the map $\rho_{\mathcal{T}, i ; h_{2}}^{\left(m ; m^{\prime}\right)}$ by (3.5), but with $i_{\mathcal{T}}\left(h_{1}, h_{2}\right)$ replaced by $\iota_{i} \in I_{k}^{+}$. Furthermore, we define the map $\rho_{\mathcal{T}, i ; i}^{\left(m ; m^{\prime}\right)}$ by replacing $h_{2}$ in (3.5) with the unique element $h(i) \in I_{k}^{+}$such that $\iota_{h(i)}=i$. We will write $x_{l ; h}(v), \rho_{\mathcal{T}, l ; h}$, and $\widetilde{F}_{l ; h} \mathcal{T}$ for $x_{j_{l} ; h}(v), \rho_{\mathcal{T}, j_{l} ; h}$, and $\widetilde{F}_{j_{l} ; h} \mathcal{T}$, respectively, whenever $l \in M_{k}$. Finally, if $d_{i} \neq 0$ for some $i \in\left[k, j_{l}\right]$, let $\sigma_{\widetilde{\mathcal{T}}, \mathcal{T}}(l)=1$; otherwise, let $\sigma_{\widetilde{\mathcal{T}}, \mathcal{T}}(l)=0$. In the former case, we put

$$
\begin{aligned}
j_{l}^{*}(\mathcal{T}) & = \begin{cases}j_{l} \in M_{k}, \\
\min \left\{i \in \widehat{I}_{k}^{-}: i \leq j_{l}, d_{h}=0 \text { for all } h \in\left(i ; j_{l}\right]\right\}, & \text { if } d_{j_{l}}=0 ;\end{cases} \\
y_{l ; \mathcal{T}}(v) & = \begin{cases}0, & \text { if } d_{j_{l}} \neq 0 ; \\
y_{h ; l}(v), & \text { if } d_{j_{l}}=0,\end{cases}
\end{aligned}
$$

where $h \in I_{k}^{+}$is given by $\iota_{h}=j_{l}^{*}(\mathcal{T})$ if $j_{l}^{*}(\mathcal{T}) \in \widehat{I}_{k}^{-}$.

Proposition 3.5 Suppose $\widetilde{\mathcal{T}}=(M, \widetilde{I} ; \tilde{j}, \underline{\widetilde{d}})$ and $\mathcal{T}=(M, I ; j, \underline{d})$ are bubble types such that $\mathcal{T}<\widetilde{\mathcal{T}}$.

(1) The spaces $\mathcal{U}_{\widetilde{\mathcal{T}}, \widetilde{\mathcal{T}}}$ and $\mathcal{U}_{\widetilde{\mathcal{T}}, \mathcal{T}}$ are smooth orbifolds, while $\overline{\mathcal{U}}_{\widetilde{\mathcal{T}}, \widetilde{\mathcal{T}}}$ is an msorbifold.

(2) There exist $\delta \in C\left(\mathcal{U}_{\widetilde{\mathcal{T}}, \mathcal{T}} ; \mathbb{R}^{+}\right)$and a map $\phi_{\widetilde{\mathcal{T}}, \mathcal{T}}: \mathcal{F}_{\widetilde{\mathcal{T}}} \mathcal{T}_{\delta} \longrightarrow \overline{\mathcal{U}}_{\widetilde{\mathcal{T}}, \widetilde{\mathcal{T}}}$ such that $\phi_{\widetilde{\mathcal{T}}, \mathcal{T}}$ is a homeomorphism onto an open neighborhood $W_{\widetilde{\mathcal{T}}, \mathcal{T}}$ of $\mathcal{U}_{\widetilde{\mathcal{T}}, \mathcal{T}}$ in $\overline{\mathcal{U}}_{\widetilde{\mathcal{T}}, \widetilde{\mathcal{T}}}$,

$$
\begin{gathered}
\phi_{\widetilde{\mathcal{T}}, \mathcal{T}}\left(\mathcal{F}_{\widetilde{\mathcal{T}}} \mathcal{T}_{\delta} \cap Y\left(\mathcal{F}_{\widetilde{\mathcal{T}}} \mathcal{T} ; I^{+}-\widetilde{I}\right)\right) \subset \partial \overline{\mathcal{U}}_{\widetilde{\mathcal{T}}, \widetilde{\mathcal{T}}}, \\
\text { and } \quad \phi_{\widetilde{\mathcal{T}}, \mathcal{T}}: \mathcal{F}_{\widetilde{\mathcal{T}}} \mathcal{T}_{\delta}-Y\left(\mathcal{F}_{\widetilde{\mathcal{T}}} \mathcal{T} ; I^{+}-\widetilde{I}\right) \longrightarrow \mathcal{U}_{\widetilde{\mathcal{T}}, \widetilde{\mathcal{T}}} \cap W_{\mathcal{T}}
\end{gathered}
$$


is an orientation-preserving diffeomorphism.

(3) Furthermore, there exist normal-neighborhood models and collections of trivializations such that the following identities are satisfied by all elements $k \in \widetilde{I}^{+}$and $(b ; v) \in \mathcal{F}_{\widetilde{\mathcal{T}}} \mathcal{T}_{\delta}-Y\left(\mathcal{F}_{\widetilde{\mathcal{T}}} \mathcal{T} ; I^{+}-\widetilde{I}\right):$

(3a) if $\widetilde{M}$ is a subset of $\widetilde{I}^{-} \sqcup M$,

$$
\mathrm{ev}_{\widetilde{\mathcal{T}}, \widetilde{M}}\left(\phi_{\widetilde{\mathcal{T}}, \mathcal{T}}(v)\right)=\mathrm{ev}_{\widetilde{\mathcal{T}}, \widetilde{M}}(b)+\varepsilon_{\widetilde{\mathcal{T}}, \widetilde{M}}(v),
$$

where $\varepsilon_{\widetilde{\mathcal{T}}, \widetilde{M}}: \mathcal{F}_{\widetilde{\mathcal{T}}} \mathcal{T}_{\delta}-Y\left(\mathcal{F}_{\widetilde{\mathcal{T}}} \mathcal{T} ; I^{+}-\widetilde{I}\right) \longrightarrow \operatorname{ev}_{\widetilde{\mathcal{T}}, \widetilde{M}}^{*} T \mathcal{X}_{\widetilde{\mathcal{T}}}(\widetilde{M})$ is a $C^{1}$-negligible map;

(3b) if $k \in \widetilde{I}^{+}$and $m \in \mathbb{Z}^{+}$,

$$
\begin{aligned}
& \mathcal{D}_{\widetilde{\mathcal{T}}, k}^{(m)} \phi_{\widetilde{\mathcal{T}}, \mathcal{T}}(v) \\
& \quad=\sum_{m^{\prime}=1}^{m}\left(\begin{array}{c}
m-1 \\
m^{\prime}-1
\end{array}\right) \sum_{h \in \chi_{k}(\mathcal{T})} x_{k ; h}(v)^{m-m^{\prime}}\left\{\mathcal{D}_{\mathcal{\mathcal { T }}, h}^{\left(m^{\prime}\right)}+\varepsilon_{\mathcal{T}, h}^{\left(m^{\prime}\right)}(v)\right\} \rho_{\mathcal{T}, k ; h}^{\left(m ; m^{\prime}\right)}(v),
\end{aligned}
$$

where each map

$$
\varepsilon_{\mathcal{T}, h}^{\left(m^{\prime}\right)}: \mathcal{F}_{\widetilde{\mathcal{T}}} \mathcal{T}_{\delta}-Y\left(\mathcal{F}_{\widetilde{\mathcal{T}}} \mathcal{T} ; I^{+}-\widetilde{I}\right) \longrightarrow \operatorname{Hom}\left(L_{h}^{\otimes m^{\prime}} \mathcal{T}, \mathrm{ev}_{k}^{*} T \mathbb{P}^{n}\right)
$$

is $C^{1}$-negligible;

(3c) if $l \in M$ and $m \in \mathbb{Z}^{+}$,

$$
\begin{aligned}
& \mathcal{D}_{\widetilde{\mathcal{T}}, l}^{(m)} \phi_{\widetilde{\mathcal{T}}, \mathcal{T}}(v) \\
& =\sigma_{\tilde{\mathcal{T}}, \mathcal{T}}(l) \sum_{m^{\prime}=m+1}^{\infty}\left(\begin{array}{c}
m^{\prime} \\
m
\end{array}\right) y_{l ; \mathcal{T}}(v)^{m^{\prime}-m}\left\{\mathcal{D}_{\mathcal{T}, j_{l}^{*}(\mathcal{T})}^{\left(m^{\prime}\right)}+\varepsilon_{\mathcal{T}, j_{l}^{*}(\mathcal{T})}^{\left(m^{\prime}\right)}(v)\right\} \rho_{\mathcal{T}, j_{l}^{*}(\mathcal{T}) ; j_{l}}^{\left(m ; m^{\prime}\right)}(v) \\
& +(-1)^{m} \sum_{m^{\prime}=1}^{\infty}\left\{\left(\begin{array}{c}
m+m^{\prime}-1 \\
m
\end{array}\right)\right. \\
& \left.\quad \sum_{h \in \chi_{l}(\mathcal{T})}\left(y_{h ; l}(v)-x_{l ; h}(v)\right)^{-\left(m+m^{\prime}\right)}\left\{\mathcal{D}_{\mathcal{\mathcal { T }}, h}^{\left(m^{\prime}\right)}+\varepsilon_{\mathcal{T}, h}^{\left(m^{\prime}\right)}(v)\right\} \rho_{\mathcal{T}, l ; h}^{\left(m ; m^{\prime}\right)}(v)\right\}
\end{aligned}
$$

where $\varepsilon_{\mathcal{T}, j_{l}^{*}(\mathcal{T})}^{\left(m^{\prime}\right)}(v)=0$ if $m^{\prime} \neq m$ and $\varepsilon_{\mathcal{T}, j_{l}^{*}(\mathcal{T})}^{(m)}$ is a $C^{1}$-negligible map on

$$
\mathcal{F}_{\widetilde{\mathcal{T}}} \mathcal{T}_{\delta}-Y\left(\mathcal{F}_{\widetilde{\mathcal{T}}} \mathcal{T} ; I^{+}-\widetilde{I}\right)
$$

The expansions (3b) and (3c) above look quite complicated. However, it is clear from the construction that they involve monomials maps between vector 
bundles. Figure [ 6 illustrates the expansion (3b) in a case when $\widetilde{I}^{+}=\left\{i^{*}\right\}$ is a single-element set and $m=1$. Note that, while the stratum $\mathcal{U}_{\mathcal{T} \mid \widetilde{\mathcal{T}}}$ of Figure 6 has codimension three in $\overline{\mathcal{U}}_{\widetilde{\mathcal{T}}}$, the section $\mathcal{D}_{\widetilde{\mathcal{T}}, i^{*}}^{(1)}$ depends only on two parameters of the normal bundle, $v_{h_{1}}$ and $v_{h_{2}} \otimes v_{h_{3}}$, at least up to negligible terms. Such bubble types $\mathcal{T}$ will always be hollow in the sense of Definition 2.14 and will not effect our computations.

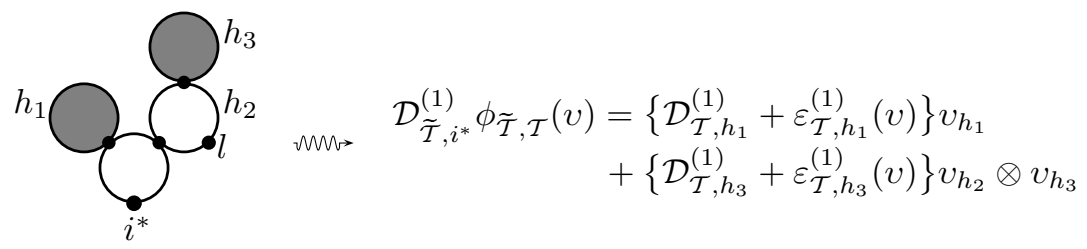

Figure 6: An example of the expansion (3b) of Proposition 3.5

One of the crucial points about the expansions (3b) and (3c) of Proposition 3.5 is that the terms that appear between the curly brackets depend on $h, j_{l}^{*}(\mathcal{T})$, and $m^{\prime}$, and not $k, l$, or $m$. Thus, by subtracting expansions of lower-derivatives with appropriate coefficients from expansions of higher-derivatives, we can get rather good estimates on the latter along the subspaces of the main stratum of a moduli space on which the former vanish; see the proofs of Lemmas 4.8 and 4.10 for example.

Statements (1) and (2) of Proposition 3.5 are basically special cases of Theorem 2.8 in [17. The map $\phi_{\widetilde{\mathcal{T}}, \mathcal{T}}$ of Proposition 3.5] is the product of the gluing maps, as constructed in Subsection 3.6 of [18, corresponding to each pair of bubble types $\mathcal{T}_{k}(\widetilde{\mathcal{T}})<\widetilde{\mathcal{T}}_{k}$. Claims $(3 \mathrm{a})$ and $(3 \mathrm{~b})$ are proved in Subsection 4.1 of [18 and in Subsection 2.5 of [17, though only a slightly weaker version of the $m=1$ case of (3b) is stated as part of Theorem 2.8 in [17. The proof of $(3 \mathrm{c})$ uses essentially the same trick as the proof of $(3 \mathrm{~b})$ in [17. The difference is that we make $\left(j_{l}, y_{l}\right)$, instead of $(k, \infty)$, a node and then use the explicit nature of the gluing map $\phi_{\widetilde{\mathcal{T}}_{k}, \mathcal{T}_{k}(\widetilde{\mathcal{T}})}$ to do integration by parts as before. The appropriate normal-neighborhood models and collections of trivializations referred to in (3) are described as follows. In (3a), we use the product of exponential maps in every component taken with respect to the family of metrics of Lemma 3.3. In (3b) and (3c), all the relevant bundles have the form $\operatorname{Hom}\left(L_{\widetilde{\mathcal{T}}, i}^{\otimes \pm m}, \mathrm{ev}_{i}^{*} T \mathbb{P}^{n}\right)$. We use parallel transport in the metric $g_{b, i}$ to identify $\phi_{\widetilde{\mathcal{T}}, \mathcal{T}}^{*} \mathrm{ev}_{i}^{*} T \mathbb{P}^{n}$ with $\pi_{\mathcal{F}_{\widetilde{\mathcal{T}}}}^{*} \mathrm{ev}_{i}^{*} T \mathbb{P}^{n}$. On the other hand, the map $\phi_{\widetilde{\mathcal{T}}_{k}, \mathcal{T}_{k}(\widetilde{\mathcal{T}})}$, con- 
structed in Subsection 3.6 of [18, is descendant from an $S^{1}$-equivariant map $\widetilde{\phi}_{\widetilde{\mathcal{T}}_{k}, \mathcal{T}_{k}(\widetilde{\mathcal{T}})}$ from a bundle over $\mathcal{B}_{\mathcal{T}_{k}(\widetilde{\mathcal{T}})}$ to $\overline{\mathcal{U}}_{\widetilde{\mathcal{T}}_{k}}^{(k)}$. This map $\widetilde{\phi}_{\widetilde{\mathcal{T}}_{k}, \mathcal{T}_{k}(\widetilde{\mathcal{T}})}$ induces an identification of the line bundles $\phi_{\widetilde{\mathcal{T}}, \mathcal{T}}^{*} L_{k} \widetilde{\mathcal{T}}$ and $\pi_{\mathcal{F}_{\tilde{\mathcal{T}}}}^{*} L_{k} \mathcal{T}$ over $\mathcal{F}_{\widetilde{\mathcal{T}}} \mathcal{T}_{\delta}$.

Remarks (1) By the construction of the map $\phi_{\widetilde{\mathcal{T}}_{k}, \mathcal{T}_{k}(\widetilde{\mathcal{T}})}$ in [18, Subsection 3.6], if $v$ is an element of $\mathcal{F}_{\widetilde{\mathcal{T}}} \mathcal{T}_{\delta}-Y\left(\mathcal{F}_{\widetilde{\mathcal{T}}} \mathcal{T} ; I^{+}-\widetilde{I}\right)$,

$$
\phi_{\widetilde{\mathcal{T}}, \mathcal{T}}(v)=\left(M, \widetilde{I} ; x(v),(\widetilde{j}, y(v)), u_{v}\right),
$$

where $y_{l}(v)=y_{k ; l}(v)$ for all $l \in M_{k}$. We use this fact in Section 4.

(2) Even in rather simple cases, the bundle $\mathcal{F}_{\widetilde{\mathcal{T}}} \mathcal{T} \longrightarrow \overline{\mathcal{U}}_{\tilde{\mathcal{T}}, \mathcal{T}}$ is not the normal bundle of $\overline{\mathcal{U}}_{\widetilde{\mathcal{T}}, \mathcal{T}}$ in $\overline{\mathcal{U}}_{\tilde{\mathcal{T}}, \tilde{\mathcal{T}}}$, as can be seen from Subsection 3.2 in [11]. Statement (2) of Proposition 3.5 only implies that the restrictions of $\mathcal{F}_{\widetilde{\mathcal{T}}} \mathcal{T}$ of the normal bundle of $\overline{\mathcal{U}}_{\widetilde{\mathcal{T}}, \mathcal{T}}$ in $\overline{\mathcal{U}}_{\widetilde{\mathcal{T}}, \widetilde{\mathcal{T}}}$ to $\mathcal{U}_{\widetilde{\mathcal{T}}, \widetilde{\mathcal{T}}}$ are isomorphic.

\section{Example 1: Rational triple-pointed curves in $\mathbb{P}^{3}$}

\subsection{Summary}

In this section, we illustrate our computational method by proving Theorem 1.2 We first describe the set $\mathcal{V}_{1}^{(2)}(\mu)$ that appears in the statement of the theorem. If $N=p+q$, we view $\mu$ as an $[N]$-tuple of constraints in $\mathbb{P}^{3}$, where

$$
[N]=\{1, \ldots, N\} .
$$

Let $I_{1}=I_{1}^{-} \sqcup I_{1}^{+}$be a two-element graded linearly ordered set. We denote the unique elements of $I_{1}^{-}$and $I_{1}^{+}$by $\widehat{0}$ and $\widetilde{1}$, respectively. Put

$$
\mathcal{T}_{0}=\left(M_{0}, I_{1}, ; j^{(0)}, d^{(0)}\right), \quad \mathcal{T}_{1}=\left(M_{1}, I_{1} ; j^{(1)}, d^{(0)}\right), \quad \mathcal{T}_{2}=\left(M_{2}, I_{1} ; j^{(2)}, d^{(0)}\right),
$$

where

$M_{0}=[N], M_{1}=M_{0} \sqcup\{\widehat{1}\}, M_{2}=M_{1} \sqcup\{\widehat{2}\} ; j_{l}^{(k)}=\widetilde{1}$ for all $l \in M_{k} ; d_{\widetilde{1}}^{(0)}=d$.

The tuples $\mathcal{T}_{0}, \mathcal{T}_{1}$, and $\mathcal{T}_{2}$ are bubble types, and we set

$$
\begin{aligned}
& \mathcal{V}_{1}^{(1)}(\mu)=\left\{b \in \mathcal{U}_{\mathcal{T}_{1}}(\mu): \mathrm{ev}_{\widetilde{1}}(b)=\operatorname{ev}_{\widehat{1}}(b)\right\}, \\
& \mathcal{U}_{\mathcal{T}_{2}}^{(1)}(\mu)=\left\{b \in \mathcal{U}_{\mathcal{T}_{2}}(\mu): \operatorname{ev}_{\widetilde{1}}(b)=\operatorname{ev}_{\widehat{1}}(b)\right\}, \\
& \mathcal{V}_{1}^{(2)}(\mu)=\left\{b \in \mathcal{U}_{\mathcal{T}_{2}}^{(1)}(\mu): \operatorname{ev}_{\widetilde{1}}(b)=\operatorname{ev}_{\widehat{2}}(b)\right\} .
\end{aligned}
$$


The cardinality of the last set is clearly six times the number of rational curves that have a triple point and pass through the tuple $\mu$ of points and lines in $\mathbb{P}^{3}$. Let $\overline{\mathcal{V}}_{1}^{(1)}(\mu)$ and $\overline{\mathcal{U}}_{\mathcal{T}_{2}}^{(1)}(\mu)$ denote the closures of the space $\mathcal{V}_{1}^{(1)}(\mu)$ in $\overline{\mathcal{U}}_{\mathcal{T}_{1}}(\mu)$ and of the space $\mathcal{U}_{\mathcal{T}_{2}}^{(1)}(\mu)$ in $\overline{\mathcal{U}}_{\mathcal{T}_{2}}(\mu)$, respectively. In the next subsection, we describe the boundary of the set $\overline{\mathcal{U}}_{\mathcal{T}_{2}}^{(1)}(\mu)$ and conclude that $\mathcal{U}_{\mathcal{T}_{2}}^{(1)}(\mu)$ is a 3-pseudovariety in $\overline{\mathcal{U}}_{\mathcal{T}_{2}}$. Thus, the map

$$
\mathrm{ev}_{\tilde{1}} \times \mathrm{ev}_{\widehat{2}}: \mathcal{U}_{\mathcal{T}_{2}}^{(1)}(\mu) \longrightarrow \mathbb{P}^{3} \times \mathbb{P}^{3}, \quad\left\{\mathrm{ev}_{\widetilde{1}} \times \mathrm{ev}_{\widehat{2}}\right\}(b)=\left(\mathrm{ev}_{\widetilde{1}}(b), \mathrm{ev}_{\widehat{2}}(b)\right),
$$

is a 6-pseudocycle in $\mathbb{P}^{3} \times \mathbb{P}^{3}$ in the sense of [10, Chapter 7] and 14, Section 1], ie the map (4.1) defines an element in $H_{6}\left(\mathbb{P}^{3} \times \mathbb{P}^{3} ; \mathbb{Z}\right)$. In particular, there is a well-defined homology-intersection number

$$
\begin{aligned}
\left\langle\left\langle\mathcal{V}_{1}^{(2)}(\mu)\right\rangle\right\rangle & \equiv\left\langle\left\langle\left\{\mathrm{ev}_{\widetilde{1}} \times \mathrm{ev}_{\widehat{2}}\right\}^{-1}\left(\Delta_{\mathbb{P}^{3} \times \mathbb{P}^{3}}\right), \overline{\mathcal{U}}_{\mathcal{T}_{2}}^{(1)}(\mu)\right\rangle\right\rangle \\
& =\sum_{r+s=3}\left\langle\left\langle\left\{\mathrm{ev}_{\widetilde{1}} \times \mathrm{ev}_{\widehat{2}}\right\}^{-1}\left(H^{r} \times H^{s}\right), \overline{\mathcal{U}}_{\mathcal{T}_{2}}^{(1)}(\mu)\right\rangle\right\rangle,
\end{aligned}
$$

where $\Delta_{\mathbb{P}^{3} \times \mathbb{P}^{3}}$ and $H^{r}$ denote the diagonal in $\mathbb{P}^{3} \times \mathbb{P}^{3}$ and a linear subspace of complex dimension $r$ in $\mathbb{P}^{3}$, respectively. However, this number is not $\left|\mathcal{V}_{1}^{(2)}(\mu)\right|$ in general. Since the map $\mathrm{ev}_{\tilde{1}} \times \mathrm{ev}_{\widehat{2}}$ is transversal to a generic submanifold $H^{r} \times H^{s}$ of $\mathbb{P}^{3} \times \mathbb{P}^{3}$, by definition,

$$
\left\langle\left\langle\mathcal{V}_{1}^{(2)}(\mu)\right\rangle\right\rangle=\left|\mathcal{V}_{1}^{(1)}\left(\mu+H^{0}\right)\right|+\left\langle a_{\widehat{0}}, \overline{\mathcal{V}}_{1}^{(1)}\left(\mu+H^{1}\right)\right\rangle+d\left\langle a_{\widehat{0}}^{2}, \overline{\mathcal{V}}_{1}^{(1)}(\mu)\right\rangle,
$$

where the spaces $\mathcal{V}_{1}^{(1)}\left(\mu+H^{0}\right)$ and $\overline{\mathcal{V}}_{1}^{(1)}\left(\mu+H^{1}\right)$ are defined as above, but with $p$ replaced by $p+1$ in the first case and with $q$ replaced by $q+1$ in the second case.

The number $\left\langle\left\langle\mathcal{V}_{1}^{(2)}(\mu)\right\rangle\right\rangle$ can also be obtained by perturbing the map ev $\mathrm{v}_{\widetilde{1}} \times \mathrm{ev}_{\widehat{2}}$. If

$$
\theta: \overline{\mathcal{U}}_{\mathcal{T}_{2}}^{(1)}(\mu) \longrightarrow \mathbb{P}^{3} \times \mathbb{P}^{3}
$$

is a small perturbation of $e v_{\widetilde{1}} \times e_{\widehat{2}}$ such that

$$
\theta^{-1}\left(\Delta_{\mathbb{P}^{3} \times \mathbb{P}^{3}}\right) \cap \partial \overline{\mathcal{U}}_{\mathcal{T}_{2}}^{(1)}(\mu)=\emptyset
$$

and $\theta \mid \mathcal{U}_{\mathcal{T}_{2}}^{(1)}(\mu)$ is smooth and transversal to $\Delta_{\mathbb{P}^{3} \times \mathbb{P}^{3}}$, then

$$
\left\langle\left\langle\mathcal{V}_{1}^{(2)}(\mu)\right\rangle\right\rangle={ }^{ \pm}\left|\theta^{-1}\left(\Delta_{\mathbb{P}^{3} \times \mathbb{P}^{3}}\right)\right| .
$$

Since $\mathcal{U}_{\mathcal{T}_{2}}^{(1)}(\mu)=\emptyset$ if $d=1$, by Lemma 3.4 the map $\mathrm{ev}_{\widetilde{1}} \times \mathrm{ev}_{\widehat{2}}$ is transversal to $\Delta_{\mathbb{P}^{3} \times \mathbb{P}^{3}}$ on $\mathcal{U}_{\mathcal{T}_{2}}^{(1)}(\mu)$. Thus, $\theta$ can be chosen so that $\theta=\mathrm{ev}_{\widetilde{1}} \times \mathrm{ev}_{\widehat{2}}$ outside of a 
very small neighborhood $W$ of $\partial \overline{\mathcal{U}}_{\mathcal{T}_{2}}^{(1)}(\mu)$. Then,

$$
\begin{aligned}
\left\langle\left\langle\mathcal{V}_{1}^{(2)}(\mu)\right\rangle\right\rangle & ={ }^{ \pm} \mid \theta^{-1}\left(\Delta_{\mathbb{P}^{3} \times \mathbb{P}^{3}} \mid\right. \\
& =\left|\mathcal{V}_{1}^{(2)}(\mu)\right|+{ }^{ \pm}\left|\left\{\operatorname{ev}_{\widetilde{1}} \times \mathrm{ev}_{\widehat{2}}\right\}^{-1}\left(\Delta_{\mathbb{P}^{3} \times \mathbb{P}^{3}}\right) \cap W\right| \\
& \equiv\left|\mathcal{V}_{1}^{(2)}(\mu)\right|+\mathcal{C}_{\partial \bar{U}_{\mathcal{T}_{2}}^{(1)}(\mu)}\left(\operatorname{ev}_{\tilde{1}} \times \mathrm{ev}_{\widehat{2}} ; \Delta_{\mathbb{P}^{3} \times \mathbb{P}^{3}}\right) .
\end{aligned}
$$

The last term above is the contribution to $\left\langle\left\langle\mathcal{V}_{1}^{(2)}(\mu)\right\rangle\right.$ from the boundary of $\overline{\mathcal{U}}_{\mathcal{T}_{2}}^{(1)}(\mu)$; it is the analogue of the term $\mathcal{C}_{\partial \overline{\mathcal{S}}}(s)$ in Proposition 2.18B If $\mathrm{ev}_{\widetilde{1}} \times \mathrm{ev}_{\widehat{2}}$ maps a stratum $\mathcal{Z}$ of $\partial \overline{\mathcal{U}}_{\mathcal{T}_{2}}^{(1)}(\mu)$ into $\Delta_{\mathbb{P}^{3} \times \mathbb{P}^{3}}$, near $\mathcal{Z}$ the map ev $\tilde{1}_{\widetilde{1}} \times \mathrm{ev}_{\widehat{2}}$ can be modeled on a section of a bundle over $\mathcal{Z}$. In Subsection 4.4 we use the topological approach of Section 2 to compute this contribution. Theorem 1.2 follows from equations (4.2) and (4.3) and Corollary 4.13.

Before concluding this subsection, we formally define the space $\overline{\mathcal{V}}_{2}^{(1,1)}(\mu)$. We do not need this space in this section, but it is used in the next section and it is natural to describe its structure along with the structure of the space $\overline{\mathcal{V}}_{1}^{(1)}(\mu)$. Let $I_{2}=I_{2}^{-} \sqcup I_{2}^{+}$be the graded linearly ordered set such that $I_{2}^{-}=\{\widehat{0}\}$ is a one-element set and $I_{2}^{+}=\{\widetilde{1}, \widetilde{2}\}$ is a two-element set. If $\mathcal{T}=\left(M_{2}, I_{2} ; j, \underline{d}\right)$ is a bubble type such that $j_{\widehat{1}}=\widetilde{1}$ and $j_{\widehat{2}}=\widetilde{2}$, put

$$
\mathcal{U}_{\mathcal{T}}^{(1)}=\left\{b \in \mathcal{U}_{\mathcal{T}}: \mathrm{ev}_{\widehat{1}}(b)=\mathrm{ev}_{\widehat{2}}(b)\right\} .
$$

Let $\overline{\mathcal{U}}_{\mathcal{T}}^{(1)}$ be the closure of $\mathcal{U}_{\mathcal{T}}^{(1)}$ in the space $\overline{\mathcal{U}}_{\mathcal{T}}$ or equivalently in $\overline{\mathcal{U}}_{\mathcal{T}, \mathcal{T}}$. We define $\mathcal{V}_{2}^{(1,1)}(\mu)$ and $\overline{\mathcal{V}}_{2}^{(1,1)}(\mu)$ to be the disjoint unions of the spaces $\mathcal{U}_{\mathcal{T}}^{(1)}(\mu)$ and $\overline{\mathcal{U}}_{\mathcal{T}}^{(1)}(\mu)$, respectively, taken over all bubble types $\mathcal{T}$ as above such that $d_{\widetilde{1}}, d_{\widetilde{2}}>0$ and $d_{\widetilde{1}}+d_{\widetilde{2}}=d$.

\subsection{On the structure of $\overline{\mathcal{U}}_{\mathcal{T}_{2}}^{(1)}(\mu), \overline{\mathcal{V}}_{1}^{(1)}(\mu)$, and similar spaces}

In this subsection, we describe the closure $\overline{\mathcal{U}}_{\mathcal{T}_{2}}^{(1)}(\mu)$ of the space $\mathcal{U}_{\mathcal{T}_{2}}^{(1)}(\mu)$ in $\overline{\mathcal{U}}_{\mathcal{T}_{2}}(\mu)$, or equivalently, in $\overline{\mathcal{U}}_{\mathcal{T}_{2}}$. The tuple $\mu$ can be arbitrary, and, in fact, $\mathbb{P}^{3}$ can be replaced by any other projective space. Lemmas 4.1 and 4.2 imply that $\mathcal{U}_{\mathcal{T}_{2}}^{(1)}(\mu)$ is a pseudovariety in $\overline{\mathcal{U}}_{\mathcal{T}_{2}}$ if $\mu$ is as in the previous subsection and is a pseudocycle in general. The two lemmas in particular describe the kinds of curves that can appear in the limit of rational one-component nodal curves, reproducing a known result in algebraic geometry, but in a fairly direct way. More importantly, we obtain a description of what happens in the limit on the 
finer level of stable maps. The analytic expansion (3b) of Proposition 3.5 plays a crucial role in the proof of the second lemma. We conclude this subsection with Lemma 4.3, which describes the structure of the space $\overline{\mathcal{V}}_{2}^{(1,1)}(\mu)$.

If $\mathcal{T}=\left(M_{2}, I ; j, \underline{d}\right)$ is a bubble type such that $\mathcal{T}<\mathcal{T}_{2}$ and $i, l \in I^{+} \cup M_{2}$, let

$$
\chi_{\mathcal{T}}(i, l)=\max \left(\chi_{\mathcal{T}, i}(l), \chi_{\mathcal{T}, l}(i)\right) ;
$$

see Subsection 3.1. Note that by continuity of the map $\mathrm{ev}_{\tilde{1}} \times \mathrm{ev}_{\hat{1}}$,

$$
\overline{\mathcal{U}}_{\mathcal{T}_{2}}^{(1)}(\mu) \subset\left\{\mathrm{ev}_{\widetilde{1}} \times \mathrm{ev}_{\widehat{1}}\right\}^{-1}\left(\Delta_{\mathbb{P}^{3} \times \mathbb{P}^{3}}\right) .
$$

Figures 7 and 8 summarize the three lemmas below. All other boundary strata are either empty or will be hollow with respect to all sections that we encounter. The map may be constant or not on the disk shaded light gray. The lines connecting two marked points indicate that the map has the same value at the two points.
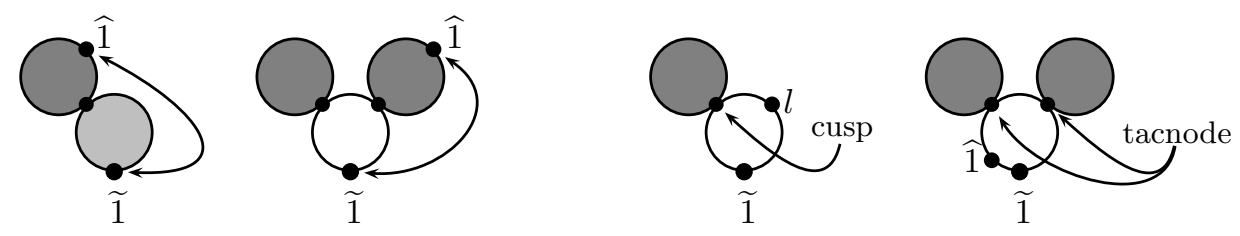

Figure 7: Some boundary strata of $\overline{\mathcal{V}}_{1}^{(1)}(\mu)$

Lemma 4.1 If $\mathcal{T}=\left(M_{2}, I ; j, \underline{d}\right)$ is a bubble type such that $\mathcal{T}<\mathcal{T}_{2}$ and $\chi_{\mathcal{T}}(\widetilde{1}, \widehat{1})>0$, the map

$$
\mathrm{ev}_{\widetilde{1}} \times \mathrm{ev}_{\widehat{1}}: \mathcal{U}_{\mathcal{T} \mid \mathcal{T}_{2}}(\mu) \longrightarrow \mathbb{P}^{3} \times \mathbb{P}^{3}
$$

is transversal to the diagonal $\Delta_{\mathbb{P}^{3} \times \mathbb{P}^{3}}$. Thus,

$$
\mathcal{U}_{\mathcal{T} \mid \mathcal{T}_{2}}^{(1)}(\mu) \equiv\left\{\operatorname{ev}_{\widetilde{1}} \times \operatorname{ev}_{\widehat{1}}\right\}^{-1}\left(\Delta_{\mathbb{P}^{3} \times \mathbb{P}^{3}}\right) \cap \mathcal{U}_{\mathcal{T}_{2}, \mathcal{T}}(\mu)
$$

is a smooth submanifold of $\mathcal{U}_{\mathcal{T} \mid \mathcal{T}_{2}}(\mu)$ of dimension less than the dimension of $\mathcal{U}_{\mathcal{T}_{2}}^{(1)}(\mu)$ with normal bundle isomorphic to $\operatorname{ev}_{\widetilde{1}}^{*} T \mathbb{P}^{3}$.

Proof The first statement is immediate from Lemma 3.4. The second claim follows from the first, since the dimension of $\mathcal{U}_{\mathcal{T} \mid \mathcal{T}_{2}}(\mu)$ is less than the dimension of $\mathcal{U}_{\mathcal{T}_{2}}(\mu)$. 
Lemma 4.2 If $\mathcal{T}$ is as in Lemma 4.1, but $\chi_{\mathcal{T}}(\widetilde{1}, \widehat{1})=0$, for every $h \in \widehat{I}^{+}$and $k \in \mathbb{Z}$ there exists a $C^{1}$-negligible map

$$
\varepsilon_{\mathcal{T}, \widehat{1} ; h}^{(k)}: \mathcal{F} \mathcal{T}_{\delta}-Y\left(\mathcal{F} \mathcal{T} ; \widehat{I}^{+}\right) \longrightarrow \operatorname{Hom}\left(L_{h}^{\otimes k} \mathcal{T}, \mathrm{ev}_{\widetilde{1}}^{*} T \mathbb{P}^{3}\right)
$$

where $\delta$ is as in Proposition 3.5, such that with notation as in Proposition 3.5 and with appropriate identifications,

$$
\begin{aligned}
\left\{\mathrm{ev}_{\tilde{1}} \times \mathrm{ev}_{\widehat{1}}\right\} & \left(\phi_{\mathcal{T}_{2}, \mathcal{T}}(v)\right) \\
= & \sum_{k=1}^{\infty} \sum_{h \in \chi_{\tilde{1}}(\mathcal{T})}\left(y_{h ; \widehat{1}}(v)-x_{\widehat{1} ; h}(v)\right)^{-k}\left\{\mathcal{D}_{\mathcal{T}_{, h}}^{(k)}+\varepsilon_{\mathcal{T}, \widehat{1} ; h}^{(k)}(v)\right\} \rho_{\mathcal{T}, \widehat{1} ; h}^{(0 ; k)}(v)
\end{aligned}
$$

for all $v \in \mathcal{F} \mathcal{T}_{\delta}-Y\left(\mathcal{F T} ; \widehat{I}^{+}\right)$. Thus, $\overline{\mathcal{U}}_{\mathcal{T}_{2}}^{(1)}(\mu) \cap \mathcal{U}_{\mathcal{T}_{2}, \mathcal{T}} \subset \mathcal{S}_{\mathcal{T} \mid \mathcal{T}_{2}}(\mu)$, where

$$
\mathcal{S}_{\mathcal{T} \mid \mathcal{T}_{2}}(\mu)=\left\{b \in \mathcal{U}_{\mathcal{T} \mid \mathcal{T}_{2}}(\mu): \sum_{h \in \chi_{\widetilde{1}}(\mathcal{T})} \mathcal{D}_{\mathcal{T}, h} v_{h}=0 \begin{array}{l}
\text { for some } v_{h} \in L_{h} \mathcal{T} \\
\text { with }\left(v_{h}\right)_{h \in \chi_{\tilde{1}}(\mathcal{T})} \neq 0
\end{array}\right\} .
$$

In particular, $\overline{\mathcal{U}}_{\mathcal{T}_{2}}^{(1)}(\mu) \cap \mathcal{U}_{\mathcal{T}_{2}, \mathcal{T}}$ is contained in a finite union of smooth submanifolds of $\mathcal{U}_{\mathcal{T}_{2}, \mathcal{T}}$ of dimension less than the dimension of $\mathcal{U}_{\mathcal{T}_{2}}^{(1)}(\mu)$.

Proof In this case, we choose a specific identification of small neighborhoods of $\Delta_{\mathbb{P}^{3} \times \mathbb{P}^{3}}$ in $\pi_{\widetilde{1}}^{*} T \mathbb{P}^{3}$ and in $\mathbb{P}^{3} \times \mathbb{P}^{3}$ :

$$
((x, x),(0, w)) \longrightarrow\left(x, \exp _{x, x} w\right),
$$

where $\exp _{x, \text {. }}$ denotes the exponential map with respect to the metric $g_{\mathbb{P}^{3}, x}$; see Lemma 3.3 Since $\chi_{\mathcal{T}}(\widetilde{1}, \widehat{1})=0$, $\operatorname{ev}_{\widetilde{1}}(b)=\operatorname{ev}_{\widehat{1}}(b)$ for all $b \in \mathcal{U}_{\mathcal{T}_{2}, \mathcal{T}}$, and thus $g_{b, \tilde{1}}=g_{b, \widehat{1}}$ for all $b \in \mathcal{U}_{\mathcal{T}_{2}, \mathcal{T}}$. The above expression for $\left\{\operatorname{ev}_{\tilde{1}} \times \operatorname{ev}_{\widehat{1}}\right\}\left(\phi_{\mathcal{T}_{2}, \mathcal{T}}(v)\right)$ is then simply the "difference" between the values of $e_{\tilde{1}}$ and $e_{\hat{1}}$ at $\phi_{\mathcal{T}_{2}, \mathcal{T}}(v)$, which is computable from (3b) of Proposition 3.5

$$
\begin{aligned}
& \left\{\operatorname{ev}_{\widetilde{1}} \times \operatorname{ev}_{\widehat{1}}\right\}\left(\phi_{\mathcal{T}_{2}, \mathcal{T}}(v)\right)=\sum_{m=1}^{\infty} y_{\widetilde{1} ; \tilde{1}}(v)^{-m} \mathcal{D}_{\mathcal{T}_{2}, \widetilde{1}}^{(m)} \phi_{\widetilde{\mathcal{T}}, \mathcal{T}}(v) \\
& =\sum_{k=1}^{\infty} \sum_{h \in \chi_{\tilde{1}}(\mathcal{T})}\left(\sum_{m=k}^{\infty}\left(\begin{array}{c}
m-1 \\
k-1
\end{array}\right) y_{\widetilde{1} ; \tilde{1}}(v)^{-m} x_{\widetilde{1} ; h}(v)^{m-k}\right)\left\{\mathcal{D}_{\mathcal{T}, h}^{(k)}+\varepsilon_{\mathcal{T}, \widehat{1} ; h}^{(k)}(v)\right\} \rho_{\mathcal{T}, \widetilde{1} ; h}^{(0 ; k)}(v) \\
& =\sum_{k=1}^{\infty} \sum_{h \in \chi_{\tilde{1}}(\mathcal{T})}\left(y_{\tilde{1} ; \tilde{1}}(v)-x_{\widetilde{1} ; h}(v)\right)^{-k}\left\{\mathcal{D}_{\mathcal{T}, h}^{(k)}+\varepsilon_{\mathcal{T}, \widehat{1} ; h}^{(k)}(v)\right\} \rho_{\mathcal{T}, \widetilde{1} ; h}^{(0 ; k)}(v)
\end{aligned}
$$

Note that $\rho_{\mathcal{T}, \widetilde{1} ; h}^{(m ; k)}=\rho_{\mathcal{T}, \widetilde{1} ; h}^{(0 ; k)}$ for all $m \in \mathbb{Z}$. The last expression in (4.4) is the same as the right-hand side of the expansion in the statement of the lemma; see 
Subsection 3.1. This sum is absolutely convergent for all $\delta$ sufficiently small, since there exists $C \in C\left(\mathcal{U}_{\mathcal{T} \mid \mathcal{T}_{2}} ; \mathbb{R}^{+}\right)$such that

$$
\begin{gathered}
\left|y_{h ; \widehat{1}}(b ; v)-x_{\widehat{1} ; h}(b ; v)\right|^{-1} \leq C(b) \quad \text { and } \\
\left|\rho_{\mathcal{T}, \widehat{1} ; h}^{(0 ; k)}(v)\right| \leq|v| \quad \text { for all } h \in \chi_{\widetilde{1}}(\mathcal{T}), \quad(b ; v) \in \mathcal{F} \mathcal{T} .
\end{gathered}
$$

The first inequality is immediate from the definitions of $y_{h ; \widehat{1}}$ and $x_{\widehat{1} ; h}$, while the second follows from the assumption $\chi_{\mathcal{T}}(\widetilde{1}, \widehat{1})=0$. The above expansion of $\left\{\mathrm{ev}_{\tilde{1}} \times \mathrm{ev}_{\widehat{1}}\right\} \circ \phi_{\mathcal{T}_{2}, \mathcal{T}}$ immediately implies that

$$
\overline{\mathcal{U}}_{\mathcal{T}_{2}}^{(1)}(\mu) \cap \mathcal{U}_{\mathcal{T}_{2}, \mathcal{T}} \subset \mathcal{S}_{\mathcal{T} \mid \mathcal{T}_{2}}(\mu)
$$

In fact, the opposite inclusion also holds, as can be seen from Lemma 3.4 and the Contraction Principle. The remaining claim of the lemma is obtained by simple dimension-counting from Lemma 3.4
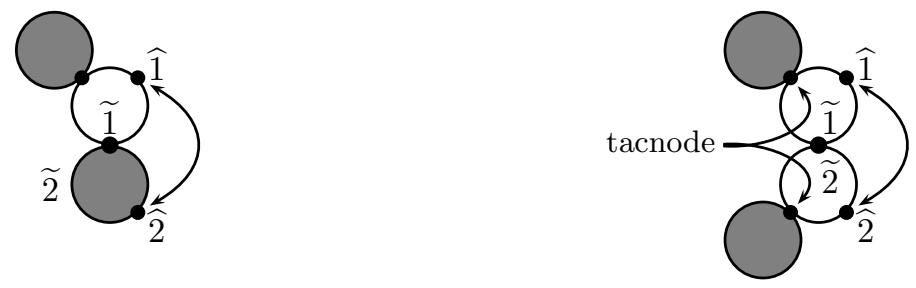

Figure 8: Some boundary strata of $\overline{\mathcal{V}}_{2}^{(1,1)}(\mu)$

Lemma 4.3 Suppose $\widetilde{\mathcal{T}}=\left(M_{2}, I_{2} ; \widetilde{j}, \widetilde{d}\right)$ and $\mathcal{T}=\left(M_{2}, I ; j, d\right)$ are bubble types such that $\widetilde{j}_{\widehat{1}}=\widetilde{1}, \widetilde{j}_{\widehat{2}}=\widetilde{2}$, and $\mathcal{T}<\widetilde{\mathcal{T}}$.

(1) If $d_{h}>0$ for some $h \in I^{+}$such that $h \leq j_{\widehat{1}}$ or $h \leq j_{\widehat{2}}$, the map

$$
\mathrm{ev}_{\widetilde{1}} \times \mathrm{ev}_{\hat{1}}: \mathcal{U}_{\mathcal{T} \mid \widetilde{\mathcal{T}}}(\mu) \longrightarrow \mathbb{P}^{3} \times \mathbb{P}^{3}
$$

is transversal to the diagonal $\Delta_{\mathbb{P}^{3} \times \mathbb{P}^{3}}$. Thus,

$$
\mathcal{U}_{\mathcal{T} \mid \widetilde{\mathcal{T}}}^{(1)}(\mu) \equiv\left\{\operatorname{ev}_{\tilde{1}} \times \mathrm{ev}_{\widehat{1}}\right\}^{-1}\left(\Delta_{\mathbb{P}^{3} \times \mathbb{P}^{3}}\right) \cap \mathcal{U}_{\mathcal{T} \mid \widetilde{\mathcal{T}}}(\mu)
$$

is a smooth submanifold of $\mathcal{U}_{\mathcal{T} \mid \widetilde{\mathcal{T}}}(\mu)$ with normal bundle isomorphic to $\mathrm{ev}_{\widetilde{1}}^{*} T \mathbb{P}^{3}$.

(2) If $d_{h}>0$ for all $h \in I^{+}$such that $h \leq j_{\widehat{1}}$ or $h \leq j_{\widehat{2}}$,

$$
\begin{aligned}
& \left\{\operatorname{ev}_{\tilde{1}} \times \operatorname{ev}_{\hat{1}}\right\}\left(\phi_{\tilde{\mathcal{T}}, \mathcal{T}}(v)\right) \\
& =\sum_{i=1,2}(-1)^{i} \sum_{k=1}^{\infty} \sum_{h \in \chi_{\tilde{i}}(\mathcal{T})}\left(y_{h, \hat{i}}(v)-x_{\widehat{i} ; h}(v)\right)^{-k}\left\{\mathcal{D}_{\mathcal{T}, h}^{(k)}+\varepsilon_{\mathcal{T}, \hat{i} ; h}^{(k)}(v)\right\} \rho_{\mathcal{T}, \hat{i} ; h}^{(0 ; k)}(v)
\end{aligned}
$$


for all $v \in \mathcal{F} \mathcal{T}_{\delta}-Y\left(\mathcal{F} \mathcal{T} ; \widehat{I}^{+}\right)$. Thus, $\overline{\mathcal{U}}_{\widetilde{\mathcal{T}}}^{(1)}(\mu) \cap \mathcal{U}_{\widetilde{\mathcal{T}}, \mathcal{T}} \subset \mathcal{S}_{\mathcal{T} \mid \widetilde{\mathcal{T}}}(\mu)$, where

$$
\begin{aligned}
& \mathcal{S}_{\mathcal{T} \mid \widetilde{\mathcal{T}}}(\mu)=\left\{b \in \mathcal{U}_{\mathcal{T} \mid \widetilde{\mathcal{T}}}(\mu): \sum_{h \in \chi_{\tilde{1}}(\mathcal{T}) \cup \chi_{\tilde{2}}(\mathcal{T})} \mathcal{D}_{\mathcal{T}, h} v_{h}=0\right. \\
& \left.\qquad \text { for some } v_{h} \in L_{h} \mathcal{T} \text { such that }\left(v_{h}\right)_{h \in \chi_{\tilde{1}} \cup \chi_{\tilde{2}}(\mathcal{T})} \neq 0\right\} .
\end{aligned}
$$

In either case, $\overline{\mathcal{U}}_{\widetilde{\mathcal{T}}}^{(1)}(\mu) \cap \mathcal{U}_{\widetilde{\mathcal{T}}, \mathcal{T}}$ is contained in a finite union of smooth submanifolds of $\mathcal{U}_{\widetilde{\mathcal{T}}, \mathcal{T}}$ of dimension less than the dimension of $\mathcal{U}_{\widetilde{\mathcal{T}}}^{(1)}(\mu)$.

Proof The proof is essentially the same as that of Lemmas 4.1 and 4.2. The only change is that in the second case we first obtain expansions for $e_{\hat{1}}-e_{\tilde{1}}$ and $e v_{\widehat{2}}-e v_{\widetilde{2}}$ and then take their difference.

\subsection{Behavior of the map $\mathrm{ev}_{\widetilde{1}} \times \mathrm{ev}_{\widehat{2}}$ near $\partial \overline{\mathcal{U}}_{\mathcal{T}_{2}}^{(1)}(\mu)$}

In this subsection, we use Lemma 3.4 and Proposition 3.5 to describe the behavior of ev ${ }_{\tilde{1}} \times e v_{\widehat{2}}$ near the boundary of the space $\overline{\mathcal{U}}_{\mathcal{T}_{2}}^{(1)}(\mu)$. We assume that $\mu$ is a tuple of points and lines in $\mathbb{P}^{3}$ as in Subsection 4.1

Lemma 4.4 If $\mathcal{T}=\left(M_{2}, I ; j, \underline{d}\right)$ is a bubble type such that $\mathcal{T}<\mathcal{T}_{2}, \chi_{\mathcal{T}}(\widetilde{1}, \widehat{2})>$ 0 , and $\chi_{\mathcal{T}}(\widehat{1}, \widehat{2})>0$,

$$
\left\{\mathrm{ev}_{\widetilde{1}} \times \mathrm{ev}_{\widehat{2}}\right\}^{-1}\left(\Delta_{\mathbb{P}^{3} \times \mathbb{P}^{3}}\right) \cap\left(\overline{\mathcal{U}}_{\mathcal{T}_{2}}^{(1)}(\mu) \cap \mathcal{U}_{\mathcal{T}_{2}, \mathcal{T}}\right)=\emptyset .
$$

Proof (1) If $\chi_{\mathcal{T}}(\widetilde{1}, \widehat{1})>0$, by Lemma 4.1.

$$
\overline{\mathcal{U}}_{\mathcal{T}_{2}}^{(1)}(\mu) \cap \mathcal{U}_{\mathcal{T}_{2}, \mathcal{T}} \subset \mathcal{U}_{\mathcal{T} \mid \mathcal{T}_{2}}^{(1)}(\mu)
$$

Since every degree-one map into $\mathbb{P}^{3}$ is injective, the map

$$
\mathrm{ev}_{\widetilde{1}} \times \mathrm{ev}_{\widehat{2}}: \mathcal{U}_{\mathcal{T} \mid \mathcal{T}_{2}}^{(1)}(\mu) \longrightarrow \mathbb{P}^{3} \times \mathbb{P}^{3}
$$

is transversal to $\Delta_{\mathbb{P}^{3} \times \mathbb{P}^{3}}$ by Lemma 3.4 Since the complex dimension of $\mathcal{U}_{\mathcal{T} \mid \mathcal{T}_{2}}^{(1)}(\mu)$ is less than three by Lemma 4.1, it follows that

$$
\left\{\mathrm{ev}_{\widetilde{1}} \times \mathrm{ev}_{\widehat{2}}\right\}^{-1}\left(\Delta_{\mathbb{P}^{3} \times \mathbb{P}^{3}}\right) \cap\left(\overline{\mathcal{U}}_{\mathcal{T}_{2}}^{(1)}(\mu) \cap \mathcal{U}_{\mathcal{T}_{2}, \mathcal{T}}\right)=\emptyset .
$$

(2) If $\chi_{\mathcal{T}}(\widetilde{1}, \widehat{1})=0$, by Lemma 4.2

$$
\overline{\mathcal{U}}_{\mathcal{T}_{2}}^{(1)}(\mu) \cap \mathcal{U}_{\mathcal{T} \mid \mathcal{T}_{2}} \subset \mathcal{S}_{\mathcal{T}_{2}, \mathcal{T}}(\mu)
$$


Since every degree-one map into $\mathbb{P}^{3}$ is an immersion, the map

$$
\mathrm{ev}_{\widetilde{1}} \times \mathrm{ev}_{\widehat{2}}: \mathcal{S}_{\mathcal{T} \mid \mathcal{T}_{2}}(\mu) \longrightarrow \mathbb{P}^{3} \times \mathbb{P}^{3}
$$

is transversal to $\Delta_{\mathbb{P}^{3} \times \mathbb{P}^{3}}$ by Lemma 3.4. Since the complex dimension of $\mathcal{S}_{\mathcal{T} \mid \mathcal{T}_{2}}(\mu)$ is less than three by Lemma 4.2, it follows that

$$
\left\{\mathrm{ev}_{\tilde{1}} \times \mathrm{ev}_{\widehat{2}}\right\}^{-1}\left(\Delta_{\mathbb{P}^{3} \times \mathbb{P}^{3}}\right) \cap\left(\overline{\mathcal{U}}_{\mathcal{T}_{2}}^{(1)}(\mu) \cap \mathcal{U}_{\mathcal{T}_{2}, \mathcal{T}}\right)=\emptyset .
$$

Lemma 4.5 If $\mathcal{T} \equiv\left(M_{2}, I ; j, \underline{d}\right)<\mathcal{T}_{2}$ is a bubble type such that $\chi_{\mathcal{T}}(\widetilde{1}, \widehat{2})=0$, $\chi_{\mathcal{T}}(\widehat{1}, \widehat{2})>0$, and $\mathcal{U}_{\mathcal{T} \mid \mathcal{T}_{2}}^{(1)}(\mu) \neq \emptyset$, then

$$
\overline{\mathcal{U}}_{\mathcal{T}_{2}}^{(1)}(\mu) \cap \mathcal{U}_{\mathcal{T}_{2}, \mathcal{T}} \subset\left\{\operatorname{ev}_{\widetilde{1}} \times \mathrm{ev}_{\widehat{2}}\right\}^{-1}\left(\Delta_{\mathbb{P}^{3} \times \mathbb{P}^{3}}\right), \quad \chi_{\mathcal{T}}(\widetilde{1}, \widehat{1})>0, \quad\left|\chi_{\widetilde{1}}(\mathcal{T})\right| \in\{1,2\} .
$$

Furthermore, there exist a rank- $\left|\chi_{\widetilde{1}}(\mathcal{T})\right|$ vector bundle $\widetilde{\mathcal{F}} \mathcal{T} \longrightarrow \mathcal{U}_{\mathcal{T}_{2}, \mathcal{T}}$, a monomials map $\rho: \mathcal{F} \mathcal{T} \longrightarrow \widetilde{\mathcal{F}} \mathcal{T}$, a section $\alpha \in \Gamma\left(\mathcal{U}_{\mathcal{T}_{2}, \mathcal{T}} ; \operatorname{Hom}\left(\widetilde{\mathcal{F}} \mathcal{T} ; \operatorname{ev}_{\widetilde{1}}^{*} T \mathbb{P}^{3}\right)\right)$, and a $C^{0}$-negligible map

$$
\varepsilon: \mathcal{F} \mathcal{T}-Y\left(\mathcal{F} \mathcal{T} ; \widehat{I}^{+}\right) \longrightarrow \operatorname{Hom}\left(\widetilde{\mathcal{F}} \mathcal{T}, \mathrm{ev}_{\widetilde{1}}^{*} T \mathbb{P}^{3}\right)
$$

such that

$$
\left\{\operatorname{ev}_{\widetilde{1}} \times \operatorname{ev}_{\widehat{2}}\right\}\left(\phi_{\mathcal{T}_{2}, \mathcal{T}}(v)\right)=\{\alpha+\varepsilon(v)\} \rho(v) \quad \text { for all } v \in \mathcal{F} \mathcal{T}_{\delta}-Y\left(\mathcal{F} \mathcal{T} ; \widehat{I}^{+}\right) .
$$

The vector-bundle map $\alpha$ is injective over $\mathcal{U}_{\mathcal{T} \mid \mathcal{T}_{2}}^{(1)}(\mu)$. Finally, if $\widehat{I}^{+}=\chi_{\widetilde{1}}(\mathcal{T}), \rho$ is the identity map, and

$$
\alpha(v)=\sum_{h \in \chi_{\tilde{1}}(\mathcal{T})}\left(y_{\widehat{2}}-x_{h}\right)^{-1} \otimes \mathcal{D}_{\mathcal{T}, h}^{(1)} v_{h} \quad \text { for all } v=\left(v_{h}\right)_{h \in \chi_{\tilde{1}}(\mathcal{T})} \in \mathcal{F} \mathcal{T} .
$$

Proof The first two claims of the first sentence are clear; the third follows by dimension-counting from Lemma 3.4. On the other hand, equation (4.4) with $\widehat{1}$ replaced by $\widehat{2}$ gives

$$
\begin{aligned}
\left\{\mathrm{ev}_{\widetilde{1}} \times \mathrm{ev}_{\widehat{2}}\right\}\left(\phi_{\mathcal{T}_{2}, \mathcal{T}}(v)\right) & \\
& =\sum_{h \in \chi_{\widetilde{1}}(\mathcal{T})}\left(y_{h ; \widehat{2}}(v)-x_{\widehat{2} ; h}(v)\right)^{-1}\left\{\mathcal{D}_{\mathcal{T}, h}^{(1)}+\widetilde{\varepsilon}_{\mathcal{T}, \widehat{2} ; h}(v)\right\} \rho_{\mathcal{T}, \hat{2} ; h}^{(0 ; 1)}(v) .
\end{aligned}
$$

Thus, we define the monomials map $\rho=\left(\rho_{h}\right)_{h \in \chi_{\tilde{1}}(\mathcal{T})}$ and the linear map $\alpha$ by:

$$
\rho_{h}(v)=\prod_{i \in\left(i_{\mathcal{T}}(\widehat{2}, h), h\right]} v_{i}, \quad \alpha\left(\left(\widetilde{v}_{h}\right)_{h \in \chi_{\widetilde{1}}(\mathcal{T})}\right)=\sum_{h \in \chi_{\widetilde{1}}(\mathcal{T})}\left(y_{h ; \widehat{2}}-x_{\widehat{2} ; h}\right)^{-1} \otimes \mathcal{D}_{\mathcal{T}, h}^{(1)} \widetilde{v}_{h},
$$


where

$$
\begin{aligned}
& x_{\widehat{2} ; h}=x_{h^{\prime}} \text { if } h^{\prime} \in\left(i_{\mathcal{T}}(\widehat{2}, h) ; h\right] \text { and } \iota_{h^{\prime}}^{+}=i_{\mathcal{T}}(\widehat{2}, h) ; \\
& y_{h, \widehat{2}}= \begin{cases}y_{\widehat{2}}, & \text { if } j_{\widehat{2}}=i_{\mathcal{T}}(\widehat{2}, h) ; \\
x_{h^{\prime}}, & \text { if } h^{\prime} \in\left(i_{\mathcal{T}}(\widehat{2}, h) ; j_{\widehat{2}}\right] \text { and } \iota_{h^{\prime}}^{+}=i_{\mathcal{T}}(\widehat{2}, h) .\end{cases}
\end{aligned}
$$

We write $b=(M, I ; x,(j, y), u)$ as before; then $x_{h}$ and $y_{l}$ are sections of a bundle over $\mathcal{U}_{\mathcal{T}_{2}, \mid \mathcal{T}}$. By Lemma 3.4 the map $\alpha$ is injective over $\mathcal{U}_{\mathcal{T} \mid \mathcal{T}_{2}}^{(1)}(\mu)$.

Lemma 4.6 If $\mathcal{T}=\left(M_{2}, I ; j, \underline{d}\right)$ is a bubble type such that $\mathcal{T}<\mathcal{T}_{2}$,

$$
\chi_{\mathcal{T}}(\widetilde{1}, \widehat{1})=\chi_{\mathcal{T}}(\widetilde{1}, \widehat{2})=\chi_{\mathcal{T}}(\widehat{1}, \widehat{2})=0,
$$

and $\mathcal{S}_{\mathcal{T} \mid \mathcal{T}_{2}}(\mu) \neq \emptyset,\left|\chi_{\tilde{1}}(\mathcal{T})\right| \in\{1,2\}$. Furthermore, the following properties hold.

(1) There exist a rank- $\left|\chi_{\widetilde{1}}(\mathcal{T})\right|$ vector bundle $\tilde{\mathcal{F}} \mathcal{T} \longrightarrow \mathcal{U}_{\mathcal{T} \mid \mathcal{T}_{2}}$, section

$$
\alpha \in \Gamma\left(\mathcal{U}_{\mathcal{T} \mid \mathcal{T}_{2}} ; \operatorname{Hom}\left(\widetilde{\mathcal{F}} \mathcal{T}, \mathrm{ev}_{\widetilde{1}}^{*} T \mathbb{P}^{3}\right)\right),
$$

and a monomials map and $C^{0}$-negligible map

$$
\rho, \varepsilon: \mathcal{F} \mathcal{T}-Y\left(\mathcal{F} \mathcal{T} ; \widehat{I}^{+}\right) \longrightarrow \widetilde{\mathcal{F}} \mathcal{T}, \operatorname{Hom}\left(\widetilde{\mathcal{F}} \mathcal{T}, \operatorname{ev}_{\widetilde{1}}^{*} T \mathbb{P}^{3}\right)
$$

such that

$$
\left\{\mathrm{ev}_{\widetilde{1}} \times \mathrm{ev}_{\widehat{2}}\right\}\left(\phi_{\mathcal{T}_{2}, \mathcal{T}}(v)\right)=\{\alpha+\varepsilon(v)\} \rho(v)
$$

for all $v \in \mathcal{F} \mathcal{T}_{\delta}$ such that $\phi_{\mathcal{T}_{2}, \mathcal{T}}(v) \in \mathcal{U}_{\mathcal{T}_{2}}^{(1)}$.

(2) If $\chi_{\tilde{1}}(\mathcal{T})=\{h\}$ is a single-element set, $\alpha$ is injective over $\mathcal{S}_{\mathcal{T} \mid \mathcal{T}_{2}}(\mu)$. If, in addition, $\widehat{I}^{+}=\chi_{\widetilde{1}}(\mathcal{T})$, then

$$
\rho\left(v_{h}\right)=v \otimes v
$$

for all $v \in \mathcal{F} \mathcal{T}$ and

$$
\alpha\left(\widetilde{v}_{h}\right)=\left(y_{\widehat{2}}-x_{h}\right)^{-2} \otimes\left(y_{\widehat{1}}-x_{h}\right)^{-1} \otimes\left(y_{\widehat{2}}-y_{\widehat{1}}\right) \otimes \mathcal{D}_{\mathcal{T}, h}^{(2)} \widetilde{v}_{h}
$$

for all $\widetilde{v} \in \tilde{\mathcal{F}} \mathcal{T} \equiv \mathcal{F} \mathcal{T}^{\otimes 2}$.

(3) If $\left|\chi_{\tilde{1}}(\mathcal{T})\right|>1$, there exist a line bundle $\mathcal{L} \longrightarrow \mathcal{U}_{\mathcal{T} \mid \mathcal{T}_{2}}$, a section

$$
\alpha_{+} \in \Gamma\left(\mathcal{U}_{\mathcal{T} \mid \mathcal{T}_{2}} ; \operatorname{Hom}\left(\widetilde{\mathcal{F}} \mathcal{T}, \mathcal{L}^{*} \otimes \operatorname{ev}_{\widetilde{1}}^{*} T \mathbb{P}^{3}\right)\right),
$$

and a monomials map and a $C^{0}$-negligible map

$$
\rho_{+}, \varepsilon_{+}: \mathcal{F} \mathcal{T}-Y\left(\mathcal{F} \mathcal{T} ; \widehat{I}^{+}\right) \longrightarrow \mathcal{L}, \operatorname{Hom}\left(\widetilde{\mathcal{F}} \mathcal{T}, \mathcal{L}^{*} \otimes \mathrm{ev}_{\tilde{1}}^{*} T \mathbb{P}^{3}\right)
$$

such that $\alpha_{+} \oplus \alpha$ is injective over $\mathcal{S}_{\mathcal{T} \mid \mathcal{T}_{2}}(\mu)$,

$$
\alpha_{+} \mid Y\left(\widetilde{\mathcal{F}} \mathcal{T},\left\{h_{1}\right\}\right) \quad \text { and } \quad \alpha_{+} \mid Y\left(\widetilde{\mathcal{F}} \mathcal{T},\left\{h_{2}\right\}\right)
$$


are onto $\operatorname{Im}\left(\alpha_{+}\right)$over $\mathcal{S}_{\mathcal{T} \mid \mathcal{T}_{2}}(\mu)$, and

$$
\left\{\mathrm{ev}_{\tilde{1}} \times \mathrm{ev}_{\widehat{1}}\right\}\left(\phi_{\mathcal{T}_{2}, \mathcal{T}}(v)\right)=\rho_{+}(v) \otimes\left\{\alpha_{+}+\varepsilon_{+}(v)\right\} \rho(v)
$$

for all $v \in \mathcal{F} \mathcal{T}_{\delta}-Y\left(\mathcal{F} \mathcal{T} ; \widehat{I}^{+}\right)$. If, in addition, $\widehat{I}^{+}=\chi_{\tilde{1}}(\mathcal{T})=\left\{h_{1}, h_{2}\right\}, \rho$ is the identity map, and

$$
\begin{gathered}
\alpha_{+}(v)=\left(y_{\widehat{1}}-x_{h_{1}}\right)^{-1} \otimes \mathcal{D}_{\mathcal{T}, h_{1}}^{(1)} v_{h_{1}}+\left(y_{\widehat{1}}-x_{h_{2}}\right)^{-1} \otimes \mathcal{D}_{\mathcal{T}, h_{2}}^{(1)} v_{h_{2}} ; \\
\alpha(v)=\left(y_{\widehat{1}}-x_{h_{2}}\right)^{-1} \otimes\left(y_{\widehat{2}}-x_{h_{1}}\right)^{-1} \otimes\left(y_{\widehat{2}}-x_{h_{2}}\right)^{-1} \otimes\left(y_{\widehat{2}}-y_{\widehat{2}}\right) \\
\otimes\left(x_{h_{1}}-x_{h_{2}}\right) \otimes \mathcal{D}_{\mathcal{T}, h_{2}}^{(1)} v_{h_{2}}
\end{gathered}
$$

for all $v=\left(v_{h_{1}}, v_{h_{2}}\right) \in \mathcal{F} \mathcal{T}_{\delta}$.

Proof (1) The first statement of this lemma follows from Lemma 3.4 by dimension-counting. If $\chi_{\widetilde{1}}(\mathcal{T})=\{h\}$ is a single-element set, the remaining claims are obtained by subtracting the expansion of $\left\{\mathrm{ev}_{\tilde{1}} \times \mathrm{ev}_{\hat{1}}\right\} \circ \phi_{\mathcal{T}_{2}, \mathcal{T}}$ given in Lemma 4.2 times

$$
\left(y_{\widetilde{1} ; \widehat{1}}(v)-x_{\widetilde{1} ; h}(v)\right)\left(y_{\tilde{1} ; \tilde{2}}(v)-x_{\widetilde{1} ; h}(v)\right)^{-1}
$$

from the corresponding expression for $\left\{\mathrm{ev}_{\tilde{1}} \times \mathrm{ev}_{\widehat{2}}\right\} \circ \phi_{\mathcal{T}_{2}, \mathcal{T}}$.

(2) If $\left|\chi_{\widetilde{1}}(\mathcal{T})\right|>1, h \in \chi_{\widetilde{1}}(\mathcal{T})$, and $l=\widehat{1}, \widehat{2}$, we put

$$
\begin{gathered}
i_{\mathcal{T}}^{*}(h)=\max \left\{i_{\mathcal{T}}(h, \widehat{1}), i_{\mathcal{T}}(h, \widehat{2})\right\} \\
i_{\mathcal{T}}^{+}(l)=\max \left\{i_{\mathcal{T}}(l, h): h \in \chi_{\widetilde{1}}(\mathcal{T})\right\}, \quad i_{\mathcal{T}}^{-}(l)=\min \left\{i_{\mathcal{T}}(l, h): h \in \chi_{\widetilde{1}}(\mathcal{T})\right\} .
\end{gathered}
$$

If $h_{1} \in \chi_{\tilde{1}}(\mathcal{T})$ is such that either $i_{\mathcal{T}}\left(h_{1}, \widehat{1}\right)=i_{\mathcal{T}}^{*}\left(h_{1}\right)$ or $i_{\mathcal{T}}\left(h_{1}, \widehat{2}\right)=i_{\mathcal{T}}(\widehat{2})$, we subtract the expansion of $\left\{\mathrm{ev}_{\tilde{1}} \times \mathrm{ev}_{\widehat{1}}\right\} \circ \phi_{\mathcal{T}_{2}, \mathcal{T}}$ given in Lemma 4.2 times

$$
\left(y_{\widetilde{1} ; \hat{1}}(v)-x_{\widetilde{1} ; h_{1}}(v)\right)\left(y_{\widetilde{1} ; \widehat{2}}(v)-x_{\widetilde{1} ; h_{1}}(v)\right)^{-1}
$$

from the corresponding expression for $\left\{\mathrm{ev}_{\widetilde{1}} \times \mathrm{ev}_{\widehat{2}}\right\} \circ \phi_{\mathcal{T}_{2}, \mathcal{T}}$ and then take the leading term.

\subsection{Computation of the number $\mathcal{C}_{\partial \overline{\mathcal{U}}_{\mathcal{I}_{2}}^{(1)}(\mu)}\left(\mathrm{ev}_{\tilde{1}} \times \mathrm{ev}_{\widehat{2}} ; \Delta_{\mathbb{P}^{3} \times \mathbb{P}^{3}}\right)$}

We are now ready to compute the term $\mathcal{C}_{\partial \overline{\mathcal{U}}_{\mathcal{T}_{2}}^{(1)}(\mu)}\left(\operatorname{ev}_{\widetilde{1}} \times \mathrm{ev}_{\widehat{2}} ; \Delta_{\mathbb{P}^{3} \times \mathbb{P}^{3}}\right)$ appearing in equation (4.3). We perturb the map $\mathrm{ev}_{\tilde{1}} \times \mathrm{ev}_{\widehat{2}}$ to a new continuous map $\theta$ on $\overline{\mathcal{U}}_{\mathcal{T}_{2}}$ such that the image of $\partial \overline{\mathcal{U}}_{\mathcal{T}_{2}}^{(1)}(\mu)$ under $\theta$ is disjoint from $\Delta_{\mathbb{P}^{3} \times \mathbb{P}^{3}}$ and $\theta \mid \mathcal{U}_{\mathcal{T}_{2}}^{(1)}(\mu)$ is smooth and transversal to $\Delta_{\mathbb{P}^{3} \times \mathbb{P}^{3}}$. In order to achieve these 
requirements, it is sufficient to perturb $\mathrm{ev}_{\widetilde{1}} \times \mathrm{ev}_{\widehat{2}}$ very slightly on a small neighborhood $W$ of

$$
\partial \overline{\mathcal{U}}_{\mathcal{T}_{2}}^{(1)}(\mu) \cap\left\{\mathrm{ev}_{\tilde{1}} \times \mathrm{ev}_{\widehat{2}}\right\}^{-1}\left(\Delta_{\mathbb{P}^{3} \times \mathbb{P}^{3}}\right) .
$$

Then,

$$
\mathcal{C}_{\partial \overline{\mathcal{U}}_{\mathcal{T}_{2}}^{(1)}(\mu)}\left(\mathrm{ev}_{\widetilde{1}} \times \mathrm{ev}_{\widehat{2}} ; \Delta_{\mathbb{P}^{3} \times \mathbb{P}^{3}}\right)={ }^{ \pm}\left|\theta^{-1}\left(\Delta_{\mathbb{P}^{3} \times \mathbb{P}^{3}}\right) \cap W\right| .
$$

Along the set $\partial \overline{\mathcal{U}}_{\mathcal{T}_{2}}^{(1)}(\mu) \cap\left\{\operatorname{ev}_{\widetilde{1}} \times \mathrm{ev}_{\widehat{2}}\right\}^{-1}\left(\Delta_{\mathbb{P}^{3} \times \mathbb{P}^{3}}\right)$, the maps ev $\widetilde{1}_{\widetilde{1}} \times \mathrm{ev}_{\widehat{2}}$ and $\theta$ can be viewed as sections of the bundle $\operatorname{ev}_{\widetilde{1}}^{*} T \mathbb{P}^{3}$. Thus, we can apply the terminology and the computational method of Section 2 to determine the number of zeros of a small perturbation of $\mathrm{ev}_{\tilde{1}} \times \mathrm{ev}_{\widehat{2}}$ near

$$
\partial \overline{\mathcal{U}}_{\mathcal{T}_{2}}^{(1)}(\mu) \cap\left\{\mathrm{ev}_{\tilde{1}} \times \mathrm{ev}_{\widehat{2}}\right\}^{-1}\left(\Delta_{\mathbb{P}^{3} \times \mathbb{P}^{3}}\right) .
$$

Of course, we cannot "cut off" the map near the entire boundary of $\overline{\mathcal{U}}_{\mathcal{T}_{2}}^{(1)}(\mu)$, as was done for vector-bundle sections in Subsection 2.3. However, the entire approach of Subsection 2.3 goes through, since $\left\{\mathrm{ev}_{\widetilde{1}} \times \mathrm{ev}_{\widehat{2}}\right\}^{-1}\left(\Delta_{\mathbb{P}^{3} \times \mathbb{P}^{3}}\right)$ is welldefined on all of the space $\overline{\mathcal{U}}_{\mathcal{T}_{2}}^{(1)}(\mu)$, and not just on $\mathcal{U}_{\mathcal{T}_{2}}^{(1)}(\mu)$.

We prove Corollary 4.13 which expresses the boundary contribution

$$
\mathcal{C}_{\partial \overline{\mathcal{U}}_{\mathcal{T}_{2}}^{(1)}(\mu)}\left(\mathrm{ev}_{\widetilde{1}} \times \mathrm{ev}_{\widehat{2}} ; \Delta_{\mathbb{P}^{3} \times \mathbb{P}^{3}}\right)
$$

in terms of level 1 numbers, by computing contributions from the individual strata $\mathcal{U}_{\mathcal{T}_{2}, \mathcal{T}}$. We split the computation into four cases, depending on whether $\chi_{\mathcal{T}}(\widetilde{1}, \widehat{2})$ and $\chi_{\mathcal{T}}(\widehat{1}, \widehat{2})$ are zero or not. By Lemma 4.4 if $\chi_{\mathcal{T}}(\widetilde{1}, \widehat{2}) \neq 0$ and $\chi_{\mathcal{T}}(\widehat{1}, \widehat{2}) \neq 0$, the space $\mathcal{U}_{\mathcal{T}_{2}, \mathcal{T}}$ makes no contribution. The case $\chi_{\mathcal{T}}(\widetilde{1}, \widehat{2})=0$ and $\chi_{\mathcal{T}}(\widehat{1}, \widehat{2}) \neq 0$ is handled in Lemma 4.7 Figure 9 shows the three possibilities for non-hollow spaces $\mathcal{U}_{\mathcal{T}_{2}, \mathcal{T}}$. In all three cases, we express the contribution from the stratum in terms of the number $N\left(\alpha_{1}\right)$ of zeros of an affine map between vector bundles. However, in two of the cases, this number is zero, basically for dimensional reasons; the remaining number is computed in Lemma 4.8. The case $\chi_{\mathcal{T}}(\widetilde{1}, \widehat{2}) \neq 0$ and $\chi_{\mathcal{T}}(\widehat{1}, \widehat{2})=0$ is symmetric to the one just considered and no separate computation is needed. The remaining case is dealt with in Lemma 4.12, Figure [11 shows the three possibilities for nonhollow spaces $\mathcal{U}_{\mathcal{T}_{2}, \mathcal{T}}$, but in all three cases the corresponding number $N\left(\alpha_{1}\right)$ is zero for dimensional reasons. In both figures, the numbers above the arrows show the multiplicity with which the corresponding number $N\left(\alpha_{1}\right)$ enters into $\mathcal{C}_{\partial \overline{\mathcal{U}}_{\mathcal{T}_{2}}^{(1)}(\mu)}\left(\mathrm{ev}_{\tilde{1}} \times \mathrm{ev}_{\widehat{2}} ; \Delta_{\mathbb{P}^{3} \times \mathbb{P}^{3}}\right)$.

Before proceeding with the actual proofs, we formally define more spaces of tuples of stable genus-zero maps that appear in the statements of Theorems 1.2 
and 1.3 and describe curves pictured in Figure 1. First, let

$$
\mathcal{S}_{1}(\mu)=\left\{b \in \mathcal{U}_{\mathcal{T}_{0}}(\mu): \mathcal{D}_{\mathcal{T}_{0}, \tilde{1}}^{(1)} b=0\right\}
$$

and let $\overline{\mathcal{S}}_{1}(\mu)$ be the closure of $\mathcal{S}_{1}(\mu)$ in $\overline{\mathcal{U}}_{\mathcal{T}_{0}}$. If $\mathcal{T}=\left(M_{0}, I_{2} ; j, \underline{d}\right)$, let

$$
\begin{aligned}
\mathcal{S}_{\mathcal{T}}(\mu)=\left\{b \in \mathcal{U}_{\mathcal{T}}(\mu):\right. & \\
& \left.\mathcal{D}_{\mathcal{T}, \widetilde{1}}^{(1)} v_{\widetilde{1}}+\mathcal{D}_{\mathcal{\mathcal { T }}, \widetilde{2}}^{(1)} v_{\widetilde{2}}=0 \text { for some }\left(b ; v_{\tilde{1}}, v_{\widetilde{2}}\right) \in L_{\widetilde{1}} \mathcal{T} \oplus L_{\widetilde{2}} \mathcal{T}-\mathcal{U}_{\mathcal{T}}\right\} .
\end{aligned}
$$

We denote by $\mathcal{S}_{2}(\mu)$ the quotient of the disjoint union of the spaces $\mathcal{S}_{\mathcal{T}}(\mu)$, taken over all bubble types $\mathcal{T}$ as above such that $d_{\widetilde{1}}, d_{\widetilde{2}}>0$ and $d_{\widetilde{1}}+d_{\widetilde{2}}=d$, by the natural action of the symmetric group $S_{2}$. Finally, if $\mathcal{T}=\left(M_{1}, I_{2} ; j, \underline{d}\right)$ is a bubble type such that $j_{\widehat{1}}=\widetilde{1}$, let

$$
\mathcal{U}_{\mathcal{T}}^{(1)}(\mu)=\left\{b \in \mathcal{U}_{\mathcal{T}}(\mu): \operatorname{ev}_{\widetilde{1}}(b)=\operatorname{ev}_{\widehat{1}}(b)\right\} .
$$

We denote by $\mathcal{V}_{2}^{(1)}(\mu)$ the disjoint union of the spaces $\mathcal{U}_{\mathcal{T}}^{(1)}(\mu)$, taken over all bubble types $\mathcal{T}$ as above such that $d_{\tilde{1}}, d_{\tilde{2}}>0$ and $d_{\tilde{1}}+d_{\tilde{2}}=d$.

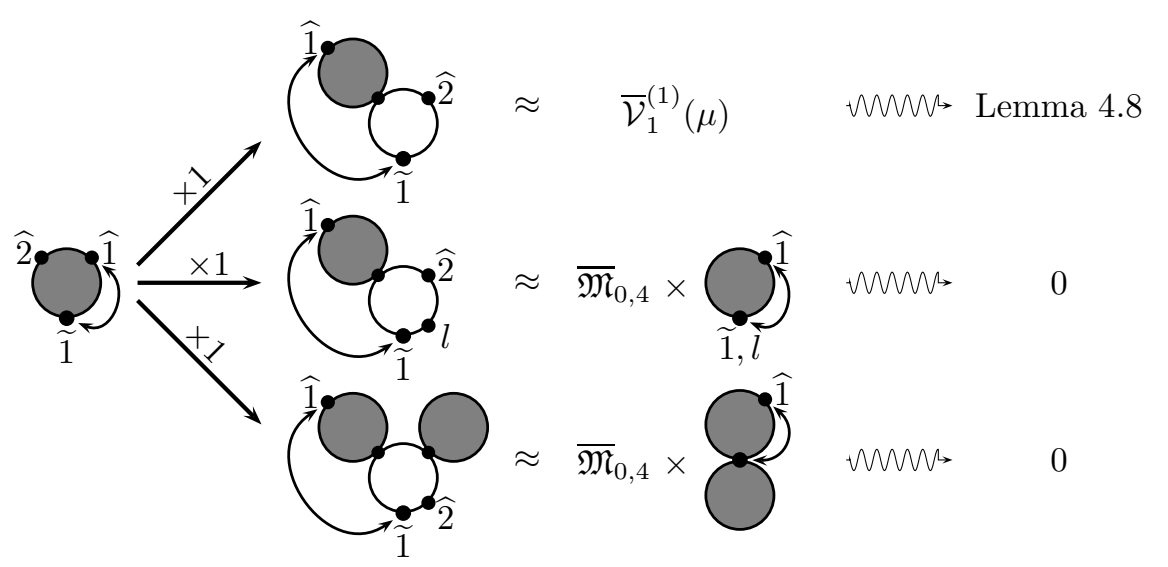

Figure 9: An outline of the proof of Lemma 4.7

Lemma 4.7 Suppose $\mathcal{T}=\left(M_{2}, I ; j, \underline{d}\right)$ is a bubble type such that $\mathcal{T}<\mathcal{T}_{2}$, $\chi_{\mathcal{T}}(\widetilde{1}, \widehat{2})=0$, and $\chi_{\mathcal{T}}(\widehat{1}, \widehat{2})>0$.

(1) If $\left|\widehat{I}^{+}\right|>\left|\chi_{\widetilde{1}}(\mathcal{T})\right|, \mathcal{U}_{\mathcal{T}}^{(1)}(\mu)$ is $\left(\mathrm{ev}_{\widetilde{1}} \times \mathrm{ev}_{\widehat{2}}, \Delta_{\mathbb{P}^{3} \times \mathbb{P}^{3}}\right)-$ hollow, and thus

$$
\mathcal{C}_{\mathcal{U}_{\mathcal{T}_{2}, \mathcal{T}}}\left(\mathrm{ev}_{\widetilde{1}} \times \mathrm{ev}_{\widehat{2}} ; \Delta_{\mathbb{P}^{3} \times \mathbb{P}^{3}}\right)=0 .
$$


(2) If $\left|\widehat{I}^{+}\right|=\left|\chi_{\widetilde{1}}(\mathcal{T})\right|=1,\left|M_{\widetilde{1}} \mathcal{T}\right| \in\{1,2\}$. If $\left|M_{\widetilde{1}} \mathcal{T}\right|=2$,

$$
\mathcal{C}_{\mathcal{U}_{\mathcal{T}_{2}, \mathcal{T}}}\left(\mathrm{ev}_{\widetilde{1}} \times \mathrm{ev}_{\widehat{2}} ; \Delta_{\mathbb{P}^{3} \times \mathbb{P}^{3}}\right)=0 \text {. }
$$

If $\left|M_{\tilde{1}} \mathcal{T}\right|=1$,

$$
\begin{aligned}
& \mathcal{C}_{\mathcal{U}_{\mathcal{T}_{2}, \mathcal{T}}}\left(\mathrm{ev}_{\widetilde{1}} \times \mathrm{ev}_{\widehat{2}} ; \Delta_{\mathbb{P}^{3} \times \mathbb{P}^{3}}\right)=\left\langle 6 a_{\widehat{0}}^{2}+4 a_{\widehat{0}} c_{1}\left(\mathcal{L}_{\widetilde{1}}^{*}\right)+c_{1}^{2}\left(\mathcal{L}_{\widetilde{1}}^{*}\right), \overline{\mathcal{V}}_{1}^{(1)}(\mu)\right\rangle+\left|\mathcal{S}_{2}(\mu)\right| \\
& -\left|\mathcal{V}_{2}^{(1)}(\mu)\right|-\left\langle 8 a_{\widehat{0}}^{2}+4 c_{1}\left(\mathcal{L}_{\widetilde{1}}^{*}\right), \overline{\mathcal{S}}_{1}(\mu)\right\rangle .
\end{aligned}
$$

(3) If $\left|\widehat{I}^{+}\right|=\left|\chi_{\widetilde{1}}(\mathcal{T})\right|=2, \mathcal{C}_{\mathcal{U}_{\mathcal{T}_{2}, \mathcal{T}}}\left(\mathrm{ev}_{\widetilde{1}} \times \mathrm{ev}_{\widehat{2}} ; \Delta_{\mathbb{P}^{3} \times \mathbb{P}^{3}}\right)=0$.

Proof (1) By Lemma 4.1, $\overline{\mathcal{U}}_{\mathcal{T}_{2}}^{(1)}(\mu) \cap \mathcal{U}_{\mathcal{T}_{2}, \mathcal{T}} \subset \mathcal{U}_{\mathcal{T} \mid \mathcal{T}_{2}}^{(1)}(\mu)$. With appropriate identifications, $\mathcal{U}_{\mathcal{T} \mid \mathcal{T}_{2}}^{(1)}(\mu)$ is the zero set of the section $\operatorname{ev}_{\mathcal{T}_{2}, M_{0}} \oplus\left(\operatorname{ev}_{\widehat{1}}-\mathrm{ev}_{\widetilde{1}}\right)$ of the bundle

$$
\mathrm{ev}_{\mathcal{T}_{2}, M_{0}}^{*} \mathcal{N} \Delta_{\mathcal{T}_{2}}(\mu) \oplus \mathrm{ev}_{\widetilde{1}}^{*} T \mathbb{P}^{3}
$$

over an open neighborhood of $\mathcal{U}_{\mathcal{T} \mid \mathcal{T}_{2}}^{(1)}(\mu)$ in $\mathcal{U}_{\mathcal{T}_{2}, \mathcal{T}}$. By Lemma 3.4, this section is transversal to the zero set, since the constraints $\mu$ are assumed to be in general position. By Proposition 3.5, there exists a $C^{1}$-negligible map

$$
\varepsilon_{-}: \mathcal{F} \mathcal{T}_{\delta}-Y\left(\mathcal{F} \mathcal{T} ; \widehat{I}^{+}\right) \longrightarrow \mathrm{ev}_{\mathcal{T}_{2}, M_{0}}^{*} \mathcal{N} \Delta_{\mathcal{T}_{2}}(\mu) \oplus \mathrm{ev}_{\widetilde{1}}^{*} T \mathbb{P}^{3}
$$

such that

$$
\left\{\mathrm{ev}_{\mathcal{T}_{2}, M_{0}} \times \mathrm{ev}_{\widetilde{1}} \times \mathrm{ev}_{\widehat{1}}\right\}\left(\phi_{\mathcal{T}_{2}, \mathcal{T}}(b ; v)\right)=\left\{\mathrm{ev}_{\mathcal{T}_{2}, M_{0}} \times \mathrm{ev}_{\widetilde{1}} \times \mathrm{ev}_{\widehat{1}}\right\}(b)+\varepsilon_{-}(b ; v)
$$

for all $(b ; v) \in \mathcal{F} \mathcal{T}_{\delta}-Y\left(\mathcal{F} \mathcal{T} ; \widehat{I}^{+}\right)$. On the other hand, by Lemma 4.5

$$
\left\{\mathrm{ev}_{\widetilde{1}} \times \mathrm{ev}_{\widehat{2}}\right\}\left(\phi_{\mathcal{T}_{2}, \mathcal{T}}(v)\right)=\{\alpha+\varepsilon(v)\} \rho(v) \in \mathrm{ev}_{\widetilde{1}}^{*} T \mathbb{P}^{3}
$$

for all $v \in \mathcal{F} \mathcal{T}_{\delta^{*}}-Y\left(\mathcal{F} \mathcal{T} ; \widehat{I}^{+}\right)$, where $\alpha$ is a linear map, which is injective over $\mathcal{U}_{\mathcal{T} \mid \mathcal{T}_{2}}^{(1)}(\mu)$, and its domain is a vector bundle of rank $\left|\chi_{\tilde{1}}(\mathcal{T})\right|$. Thus, if $\left|\widehat{I}^{+}\right|>\left|\chi_{\widetilde{1}}(\mathcal{T})\right|$, then $\mathcal{U}_{\mathcal{T} \mid \mathcal{T}_{2}}^{(1)}(\mu)$ is $\left(\operatorname{ev}_{\widetilde{1}} \times \mathrm{ev}_{\widehat{2}}, \Delta_{\mathbb{P}^{3} \times \mathbb{P}^{3}}\right)$-hollow, and

$$
\mathcal{C}_{\mathcal{U}_{\mathcal{T}_{2}, \mathcal{T}}}\left(\mathrm{ev}_{\widetilde{1}} \times \mathrm{ev}_{\widehat{2}} ; \Delta_{\mathbb{P}^{3} \times \mathbb{P}^{3}}\right)=0
$$

by Proposition 2.18B, or Lemma 2.20, and Lemma 4.1

(2) On the other hand, if $\left|\widehat{I}^{+}\right|=\left|\chi_{\tilde{1}}(\mathcal{T})\right|$, by the above and Lemma 4.5, $\mathcal{U}_{\mathcal{T} \mid \mathcal{T}_{2}}^{(1)}(\mu)$ is $\left(\mathrm{ev}_{\widetilde{1}} \times \mathrm{ev}_{\widehat{2}}, \Delta_{\mathbb{P}^{3} \times \mathbb{P}^{3}}\right)$-regular, and by Proposition 2.18B and rescaling of the linear map,

$$
\begin{gathered}
\mathcal{C}_{\mathcal{U}_{\mathcal{T}_{2}, \mathcal{T}}}\left(\mathrm{ev}_{\tilde{1}} \times \mathrm{ev}_{\widehat{2}} ; \Delta_{\mathbb{P}^{3} \times \mathbb{P}^{3}}\right)=N(\alpha), \quad \text { where } \\
\alpha \in \Gamma\left(\mathcal{U}_{\mathcal{T} \mid \mathcal{T}_{2}}^{(1)}(\mu) ; \operatorname{Hom}\left(\mathcal{F} \mathcal{T}, \operatorname{ev}_{\widetilde{1}}^{*} T \mathbb{P}^{3}\right)\right), \quad \alpha(v)=\sum_{h \in \chi_{\tilde{1}}(\mathcal{T})}\left(y_{\widehat{2}}-x_{h}\right)^{-1} \otimes \mathcal{D}_{\mathcal{T}, h}^{(1)} v_{h} .
\end{gathered}
$$


provided $\alpha$ is a regular section, as is implied by what follows. Since the map

$$
\mathcal{F} \mathcal{T} \equiv \bigoplus_{h \in \chi_{\tilde{1}}(\mathcal{T})} L_{\widetilde{1}}^{*} \mathcal{T} \otimes L_{h} \mathcal{T} \longrightarrow \mathcal{F}=\bigoplus_{h \in \chi_{\tilde{1}}(\mathcal{T})} L_{h} \mathcal{T}, \quad v_{h} \longrightarrow\left(y_{\widehat{2}}-x_{h}\right)^{-1} \otimes v_{h},
$$

is simply a rescaling of factors over $\mathcal{U}_{\mathcal{T}_{2}}(\mu)$,

$$
\begin{gathered}
\mathcal{C}_{\mathcal{U}_{\mathcal{T} \mid \mathcal{T}_{2}}}\left(\mathrm{ev}_{\widetilde{1}} \times \mathrm{ev}_{\widehat{2}} ; \Delta_{\mathbb{P}^{3} \times \mathbb{P}^{3}}\right)=N\left(\alpha^{\prime}\right), \quad \text { where } \\
\alpha^{\prime} \in \Gamma\left(\overline{\mathcal{U}}_{\mathcal{T} \mid \mathcal{T}_{2}}^{(1)}(\mu) ; \operatorname{Hom}\left(\mathcal{F}, \operatorname{ev}_{\widetilde{1}}^{*} T \mathbb{P}^{3}\right)\right), \quad \alpha^{\prime}(v)=\sum_{h \in \chi_{\tilde{1}}(\mathcal{T})} \mathcal{D}_{\mathcal{T}, h}^{(1)} v_{h} .
\end{gathered}
$$

Note that with respect to the decomposition (3.4), shown in Figure 9, the linear map $\alpha^{\prime}$ comes entirely from the second factor. Thus, if the first factor is positive-dimensional, $N\left(\alpha^{\prime}\right)=0$, ie

$$
\mathcal{C}_{\mathcal{U}_{\mathcal{T}_{2}, \mathcal{T}}}\left(\mathrm{ev}_{\widetilde{1}} \times \mathrm{ev}_{\widehat{2}} ; \Delta_{\mathbb{P}^{3} \times \mathbb{P}^{3}}\right)=0
$$

unless $\left|\chi_{\widetilde{1}}(\mathcal{T})\right|=1$ and $\left|M_{\tilde{1}} \mathcal{T}\right|=1$. If $\left|\chi_{\widetilde{1}}(\mathcal{T})\right|=1$ and $\left|M_{\tilde{1}} \mathcal{T}\right|=1$, we conclude that

$$
\begin{gathered}
\mathcal{C}_{\mathcal{U}_{\mathcal{T}_{2}, \mathcal{T}}}\left(\operatorname{ev}_{\widetilde{1}} \times \mathrm{ev}_{\widehat{2}} ; \Delta_{\mathbb{P}^{3} \times \mathbb{P}^{3}}\right)=N\left(\alpha_{1}\right), \quad \text { where } \\
\alpha_{1} \in \Gamma\left(\overline{\mathcal{V}}_{1}^{(1)}(\mu) ; \operatorname{Hom}\left(L_{\widetilde{1}} \mathcal{T}_{1}, \operatorname{ev}_{\widehat{0}}^{*} T \mathbb{P}^{3}\right)\right), \quad \alpha_{1}(v)=\mathcal{D}_{\mathcal{T}_{1}, \tilde{1}}^{(1)} v .
\end{gathered}
$$

The number $N\left(\alpha_{1}\right)$ is computed below.

Lemma 4.8 If $\alpha_{1} \in \Gamma\left(\overline{\mathcal{V}}_{1}^{(1)}(\mu) ; \operatorname{Hom}\left(L_{\widetilde{1}}, \operatorname{ev}_{\widehat{0}}^{*} T \mathbb{P}^{3}\right)\right)$ is given by $\alpha_{1}=\mathcal{D}_{\mathcal{T}_{1}, \tilde{1}}^{(1)}$,

$$
\begin{aligned}
N\left(\alpha_{1}\right)=\left\langle 6 a_{\widehat{0}}^{2}+4 a_{\widehat{0}} c_{1}\left(\mathcal{L}_{\widetilde{1}}^{*}\right)+c_{1}^{2}\left(\mathcal{L}_{\widetilde{1}}^{*}\right), \overline{\mathcal{V}}_{1}^{(1)}(\mu)\right\rangle+ & \left|\mathcal{S}_{2}(\mu)\right|-\left|\mathcal{V}_{2}^{(1)}(\mu)\right| \\
& -\left\langle 8 a_{\widehat{0}}^{2}+4 c_{1}\left(\mathcal{L}_{\widetilde{1}}^{*}\right), \overline{\mathcal{S}}_{1}(\mu)\right\rangle .
\end{aligned}
$$

Proof (1) Since $\alpha_{1}$ does not vanish on $\mathcal{V}_{1}^{(1)}(\mu)$ by Lemma 3.4 by Propositions $2.18 \mathrm{~A}$ and $2.18 \mathrm{~B}$.

$$
N\left(\alpha_{1}\right)=\left\langle 6 a_{\widehat{0}}^{2}+4 a_{\widehat{0}} c_{1}\left(L_{\widetilde{1}}^{*}\right)+c_{1}^{2}\left(L_{\widetilde{1}}^{*}\right), \overline{\mathcal{V}}_{1}^{(1)}(\mu)\right\rangle-\mathcal{C}_{\partial \overline{\mathcal{V}}_{1}^{(1)}(\mu)}\left(\alpha_{1}^{\perp}\right),
$$

where $\alpha_{1}^{\perp}$ denotes the composition of $\alpha_{1}$ with the projection $\pi_{\bar{\nu}_{1}}^{\perp}$ onto the quotient $\mathcal{O}_{1}$ of $\operatorname{ev}_{\widehat{0}}^{*} T \mathbb{P}^{3}$ by a generic trivial line subbundle $\mathbb{C} \bar{\nu}_{1}$. Figure 10 shows the five types of boundary strata that are not $\alpha_{1}^{\perp}$-hollow. Contributions from the first two are computed in (2) below, from the following two in (3), and from the last one in (4) below. 


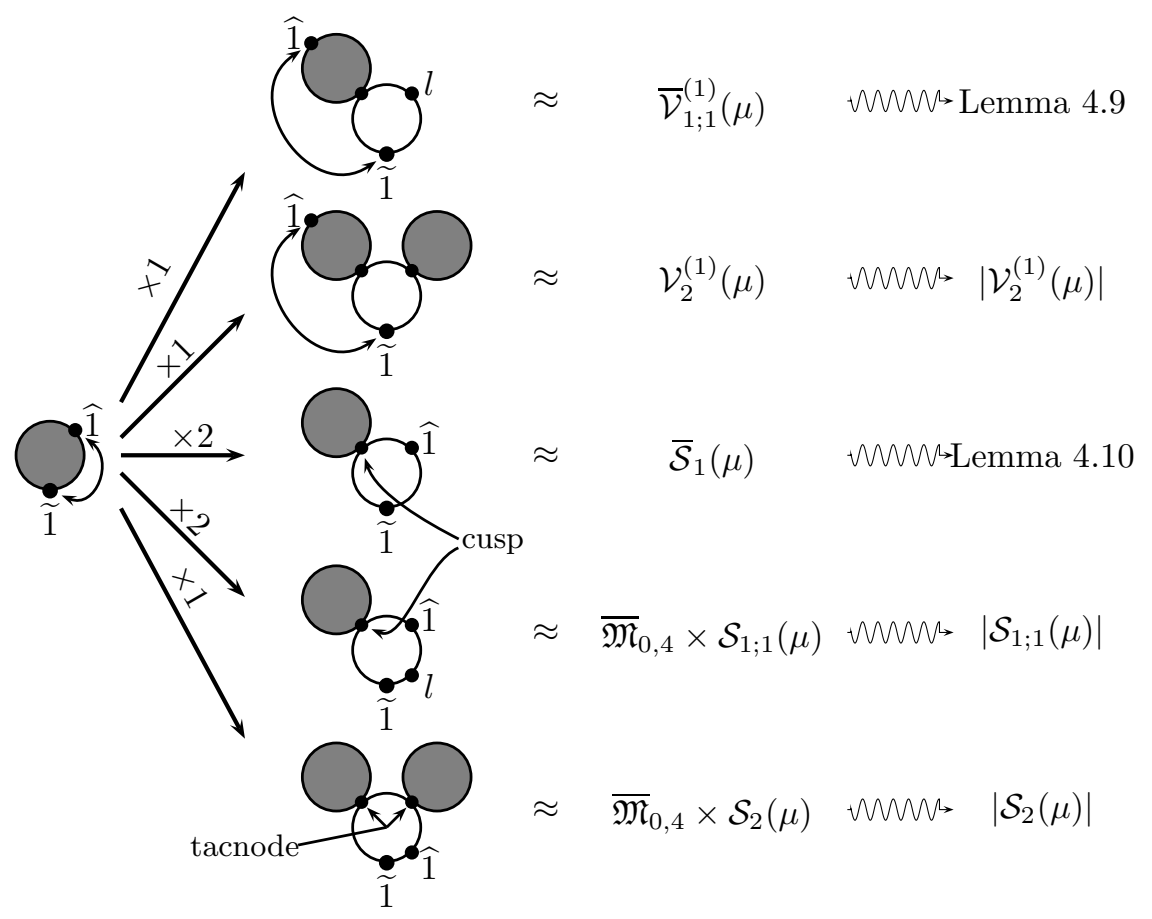

Figure 10: An outline of the proof of Lemma 4.8

(2) If $\mathcal{T}<\mathcal{T}_{1}$ and $\chi_{\mathcal{T}}(\widetilde{1}, \widehat{1})>0, \overline{\mathcal{V}}_{1}^{(1)}(\mu) \cap \mathcal{U}_{\mathcal{T}_{1}, \mathcal{T}} \subset \mathcal{U}_{\mathcal{T} \mid \mathcal{T}_{1}}^{(1)}(\mu)$ by Lemma 4.1 By Proposition 3.5.

$$
\begin{gathered}
\mathcal{D}_{\mathcal{T}_{1}, \tilde{1}}^{(1)}\left(\phi \mathcal{T}_{1}, \mathcal{T}(v)\right)=\sum_{h \in \chi_{\tilde{1}}(\mathcal{T})}\left(\mathcal{D}_{\mathcal{T}, h}^{(1)}+\varepsilon_{h}(v)\right) \rho_{h}(v), \quad \text { where } \\
\rho_{h}(v)=\prod_{i \in(\widetilde{1}, h]} v_{i}, \quad \text { for all } v \in \mathcal{F} \mathcal{T}_{\delta^{*}}-Y\left(\mathcal{F} \mathcal{T} ; \widehat{I}^{+}\right)
\end{gathered}
$$

and for some $C^{0}$-negligible maps

$$
\varepsilon_{h}: \mathcal{F} \mathcal{T}_{\delta^{*}}-Y\left(\mathcal{F} \mathcal{T} ; \widehat{I}^{+}\right) \longrightarrow \operatorname{Hom}\left(L_{h} \mathcal{T}, \mathrm{ev}_{\widehat{0}}^{*} T \mathbb{P}^{3}\right)
$$

Let $\widetilde{\mathcal{F}}_{h} \mathcal{T}$ denote the line bundle determined by $\rho_{h}$. By Lemma 3.4 the map

$$
\widetilde{\mathcal{F}} \mathcal{T} \equiv \bigoplus_{h \in \chi_{\tilde{1}}(\mathcal{T})} \widetilde{\mathcal{F}}_{h} \mathcal{T} \longrightarrow L_{\widetilde{1}}^{*} \mathcal{T} \otimes \operatorname{ev}_{\widehat{0}}^{*} T \mathbb{P}^{3}, \quad\left(\widetilde{v}_{h}\right)_{h \in \chi_{\tilde{1}}(\mathcal{T})} \longrightarrow \sum_{h \in \chi_{\tilde{1}}(\mathcal{T})} \mathcal{D}_{\mathcal{T}, h}^{(1)} \widetilde{v}_{h}
$$


is injective over $\mathcal{U}_{\mathcal{T} \mid \mathcal{T}_{1}}^{(1)}(\mu)$. If $\bar{\nu}_{1}$ is generic, the same is true of the map

$$
\alpha_{2}^{\prime}: \tilde{\mathcal{F}} \mathcal{T} \longrightarrow L_{\widetilde{1}}^{*} \mathcal{T} \otimes \mathcal{O}_{1}, \quad\left\{\alpha_{2}^{\prime}(\widetilde{v})\right\}(v)=\pi_{\bar{\nu}_{1}}^{\perp} \sum_{h \in \chi_{\tilde{1}}(\mathcal{T})} \mathcal{D}_{\mathcal{T}, h}^{(1)} \widetilde{v}_{h} \otimes v .
$$

By the same argument as in (1) of the proof of Lemma 4.7 .

$$
\mathcal{C}_{\mathcal{U}_{\mathcal{T}_{1}, \mathcal{T}}}\left(\alpha_{1}^{\perp}\right)=0 \quad \text { unless } \quad \widehat{I}^{+}=\chi_{\widetilde{1}}(\mathcal{T}) .
$$

If $\widehat{I}^{+}=\chi_{\widetilde{1}}(\mathcal{T})$, by dimension-counting either

$$
\begin{gathered}
\left|\widehat{I}^{+}\right|=\left|\chi_{\widetilde{1}}(\mathcal{T})\right|=1 \quad \text { and } \quad\left|M_{\widetilde{1}} \mathcal{T}\right|=1 \quad \text { or } \\
\left|\widehat{I}^{+}\right|=\left|\chi_{\widetilde{1}}(\mathcal{T})\right|=2 \quad \text { and } \quad\left|M_{\widetilde{1}} \mathcal{T}\right|=0 .
\end{gathered}
$$

In the second case, the map $\alpha_{2}^{\prime}$ is an isomorphism on every fiber of $\mathcal{F} \mathcal{T}$ over the finite set $\mathcal{U}_{\mathcal{T} \mid \mathcal{T}_{1}}^{(1)}(\mu)$. Thus, by Proposition 2.18B

$$
\mathcal{C}_{\mathcal{U}_{\mathcal{T}_{1}, \mathcal{T}}}\left(\alpha_{1}^{\perp}\right)=\left|\mathcal{U}_{\mathcal{T} \mid \mathcal{T}_{1}}^{(1)}(\mu)\right| .
$$

Note that the sum of the numbers $\left|\mathcal{U}_{\mathcal{T} \mid \mathcal{T}_{1}}^{(1)}(\mu)\right|$, taken over all bubble types $\mathcal{T}<\mathcal{T}_{1}$ such that

$$
\left|\widehat{I}^{+}\right|=\left|\chi_{\widetilde{1}}(\mathcal{T})\right|=2 \quad \text { and } \quad\left|M_{\widetilde{1}} \mathcal{T}\right|=0,
$$

is $\left|\mathcal{V}_{2}^{(1)}(\mu)\right|$. On the other hand, if $\widehat{I}^{+}=\chi_{\widetilde{1}}(\mathcal{T})=\{h\}$ is a single-element set and $\left|M_{\tilde{1}} \mathcal{T}\right|=1$, ie $\mathcal{T}=\mathcal{T}_{1}(l)$ for some $l \in[N]$, by Proposition 2.18B and the decomposition (3.4),

$$
\mathcal{C}_{\mathcal{U}_{\mathcal{T} \mid \mathcal{T}_{1}}^{(1)}(\mu)}\left(\alpha_{1}^{\perp}\right)=N\left(\alpha_{2}\right), \quad \text { where } \quad \alpha_{2} \in \Gamma\left(\overline{\mathcal{U}}_{\mathcal{T}_{1} / l}^{(1)}(\mu) ; \operatorname{Hom}\left(L_{h}, \mathcal{O}_{1}\right)\right)
$$

is the map induced by $\alpha_{2}^{\prime}$, ie the composition of $\mathcal{D}_{\mathcal{T}_{1} / l, h}^{(1)}$ with the projection $\pi_{\bar{\nu}_{1}}^{\perp}$ onto the quotient $\mathcal{O}_{1}$ of $\operatorname{ev}_{\widehat{0}}^{*} T \mathbb{P}^{3}$ by a generic line subbundle $\mathbb{C} \bar{\nu}_{1}$. Thus, by Lemma 4.9

$$
\sum_{l \in[N]} \mathcal{C}_{\mathcal{U}_{\mathcal{T}_{1}(l) \mid \mathcal{T}_{1}}}\left(\alpha_{1}^{\perp}\right)=\left\langle 4 a_{\widehat{0}}+c_{1}\left(L_{\widetilde{1}}^{*}\right), \overline{\mathcal{V}}_{1 ; 1}^{(1)}(\mu)\right\rangle-2\left|\mathcal{S}_{1 ; 1}(\mu)\right| .
$$

Summing up the above contributions, we find that

$$
\sum_{\chi_{\mathcal{T}}(\widetilde{1}, \widehat{1})>0} \mathcal{C}_{\mathcal{U}_{\mathcal{T}_{1}, \mathcal{T}}}\left(\alpha_{1}^{\perp}\right)=\left|\mathcal{V}_{2}^{(1)}(\mu)\right|+\left\langle 4 a_{\widehat{0}}+c_{1}\left(L_{\widetilde{1}}^{*}\right), \overline{\mathcal{V}}_{1 ; 1}^{(1)}(\mu)\right\rangle-2\left|\mathcal{S}_{1 ; 1}(\mu)\right| .
$$

In (4.6), $\mathcal{S}_{1 ; 1}(\mu)$ denotes the disjoint union of the sets

$$
\begin{aligned}
& \mathcal{S}_{\mathcal{T}_{0 ; l}}(\mu)=\left\{b \in \mathcal{U}_{\mathcal{T}_{0 ; l}}(\mu): \mathcal{D}_{\mathcal{T}_{0 ; l}, \tilde{1}}^{(1)} b=0\right\}, \quad \text { where } \\
& \mathcal{T}_{0 ; l}=\left(M_{0}-\{l\}, I_{1}(l) ; j, d\right), \quad I_{1}(l)=\{\widehat{0}=l\} \sqcup I_{1}^{+},
\end{aligned}
$$


taken over all $l \in[N]$. The space $\mathcal{V}_{1 ; 1}^{(1)}(\mu)$ is the disjoint union of the sets

$$
\begin{gathered}
\mathcal{U}_{\mathcal{T}_{1 ; l}}^{(1)}(\mu)=\left\{b \in \mathcal{U}_{\mathcal{T}_{1 ; l}}(\mu): \operatorname{ev}_{\widetilde{1}}(b)=\operatorname{ev}_{\widehat{1}}(b)\right\}, \quad \text { where } \\
\mathcal{T}_{1 ; l}=\left(M_{1}-\{l\}, I_{1}(l) ; j, d\right),
\end{gathered}
$$

taken over all $l \in[N]$. As usual, $\overline{\mathcal{V}}_{1 ; 1}^{(1)}(\mu)$ denotes the closure of $\mathcal{V}_{1 ; 1}^{(1)}(\mu)$ inside of a union of moduli spaces of stable maps. More geometrically, the image of every element of $\mathcal{S}_{1 ; 1}(\mu)$ (of $\mathcal{V}_{1 ; 1}^{(1)}(\mu)$ ) has a cusp (a node) at one of the constraints $\mu_{1}, \ldots, \mu_{N}$.

(3) If $\mathcal{T}<\mathcal{T}_{1}$ and $\chi_{\mathcal{T}}(\widetilde{1}, \hat{1})=0, \overline{\mathcal{V}}_{1}^{(1)}(\mu) \cap \mathcal{U}_{\mathcal{T}_{1}, \mathcal{I}} \subset \mathcal{S}_{\mathcal{T} \mid \mathcal{T}_{1}}(\mu)$ by Lemma 4.2. On the other hand, by Lemma 3.4. $\mathcal{S}_{\mathcal{T} \mid \mathcal{T}_{1}}(\mu)=\emptyset$ unless $\left|\chi_{\widetilde{1}}(\mathcal{T})\right| \in\{1,2\}$. Suppose $\chi_{\widetilde{1}}(\mathcal{T})=\{h\}$ is a single-element set. With appropriate identifications, $\mathcal{S}_{\mathcal{T} \mid \mathcal{T}_{1}}(\mu)$ is the zero set of the section $\operatorname{ev}_{\mathcal{T}_{1}, M_{0}} \oplus \mathcal{D}_{\mathcal{T}, h}^{(1)}$ of the bundle

$$
\mathrm{ev}_{\mathcal{T}_{1}, M_{0}}^{*} \mathcal{N} \Delta_{\mathcal{T}_{1}}(\mu) \oplus L_{h}^{*} \otimes \mathrm{ev}_{\widehat{0}}^{*} T \mathbb{P}^{3}
$$

defined over a neighborhood of $\mathcal{S}_{\mathcal{T} \mid \mathcal{T}_{1}}(\mu)$ in $\mathcal{U}_{\mathcal{T}_{1}, \mathcal{T}}$. By Lemma 3.4 this section is transverse to the zero set. By Proposition 3.5] and Lemma 4.2 .

$$
\begin{gathered}
\operatorname{ev}_{\mathcal{T}_{1}, M_{0}}\left(\phi_{\mathcal{T}_{1}, \mathcal{T}}(b ; v)\right)=\operatorname{ev}_{\mathcal{T}_{1}, M_{0}}(b)+\varepsilon_{-; 1}(b ; v), \\
\left\{\operatorname{ev}_{\tilde{1}} \times \operatorname{ev}_{\widehat{1}}\right\}\left(\phi_{\mathcal{T}_{1}, \mathcal{T}}(v)\right)=\left(y_{h ; \hat{1}}-x_{h ; \hat{1}}\right)^{-1} \otimes\left\{\mathcal{D}_{\mathcal{T}, h}^{(1)}+\varepsilon_{-; 2}(v)\right\} \rho_{\mathcal{T}, \widehat{1} ; h}^{(0 ; 1)}(v)
\end{gathered}
$$

for all $(b ; v) \in \mathcal{F} \mathcal{T}_{\delta}-Y\left(\mathcal{F} \mathcal{T} ; \widehat{I}^{+}\right)$and some $C^{1}$-negligible maps

$$
\varepsilon_{-; 1}, \varepsilon_{-; 2}: \mathcal{F} \mathcal{T}_{\delta}-Y\left(\mathcal{F} \mathcal{T} ; \widehat{I}^{+}\right) \longrightarrow \mathrm{ev}_{\mathcal{T}_{1}, M_{0}}^{*} \mathcal{N} \Delta_{\mathcal{T}_{1}}(\mu), L_{h}^{*} \otimes \mathrm{ev}_{\widehat{0}}^{*} T \mathbb{P}^{3}
$$

On the other hand, subtracting $\left(y_{\tilde{1} ; \hat{1}}(v)-x_{\widetilde{1} ; h}(v)\right)$ times the expansion of $\left\{\mathrm{ev}_{\tilde{1}} \times \mathrm{ev}_{\hat{1}}\right\} \circ \phi_{\mathcal{T}_{1}, \mathcal{T}}$ in Lemma 4.2 from the expansion of $\mathcal{D}_{\mathcal{T}_{1}, \widetilde{1}^{\circ}}^{(1)} \circ \phi_{\mathcal{T}_{1}, \mathcal{T}}$ in (3b) of Proposition 3.5 we obtain

$$
\begin{aligned}
& \mathcal{D}_{\mathcal{T}_{1},{ }_{1}}^{(1)} \phi_{\mathcal{T}_{1}, \mathcal{T}}(v)=-\left(y_{h ; \hat{1}}-x_{\widehat{1} ; h}\right) \otimes\left\{\mathcal{D}_{\mathcal{T}, h}^{(2)}+\varepsilon(v)\right\} \rho(v), \\
& \text { where } \quad \rho(v)=\prod_{i \in\left(\chi_{\mathcal{T}}(\widehat{1}, h), h\right]} v_{i}^{\otimes 2} \otimes \prod_{i \in\left(\widetilde{1}, \chi_{\mathcal{T}}(\widehat{1}, h)\right]} v_{i},
\end{aligned}
$$

for all $v \in \mathcal{F} \mathcal{T}_{\delta}$ such that $\phi_{\mathcal{T}_{1}, \mathcal{T}}(v) \in \mathcal{U}_{\mathcal{T}_{1}}^{(1)}(\mu)$ and for some $C^{0}$-negligible map

$$
\varepsilon: \mathcal{F} \mathcal{T}_{\delta}-Y\left(\mathcal{F} \mathcal{T} ; \widehat{I}^{+}\right) \longrightarrow L_{h}^{* \otimes 2} \otimes \mathrm{ev}_{\widehat{0}}^{*} T \mathbb{P}^{3} .
$$

By Lemma 3.4. $\mathcal{D}_{\mathcal{T}, h}^{(2)}$ does not vanish over $\mathcal{S}_{\mathcal{T} \mid \mathcal{T}_{1}}(\mu)$, and neither does the linear map

$$
\alpha_{2}^{\prime}: \mathcal{F}_{h} \mathcal{T}^{\otimes 2} \longrightarrow L_{\widetilde{1}}^{*} \mathcal{T} \otimes \mathcal{O}_{1}, \quad\left\{\alpha_{2}^{\prime}(\widetilde{v})\right\}(v)=\pi_{\bar{\nu}_{1}}^{\perp} \mathcal{D}_{\mathcal{T}, h}^{(2)} \widetilde{v} \otimes v
$$


provided $\bar{\nu}_{1}$ is generic. Thus, $\mathcal{S}_{\mathcal{T} \mid \mathcal{T}_{1}}(\mu)$ is $\alpha_{1}^{\perp}$-hollow unless $\widehat{I}^{+}=\chi_{\widetilde{1}}(\mathcal{T})$. On the other hand, if $\widehat{I}^{+}=\chi_{\widetilde{1}}(\mathcal{T})$, by Proposition $2.18 \mathrm{~B}$, a rescaling of the linear map, and the decomposition (3.4),

$$
\begin{gathered}
\mathcal{C}_{\mathcal{U}_{\mathcal{1}_{1}, \mathcal{T}}(\mu)}\left(\alpha_{1}^{\perp}\right)=2 N\left(\alpha_{2}\right), \quad \text { where } \\
\alpha_{2} \in \Gamma\left(\overline{\mathfrak{M}}_{\{\widetilde{1}, h\} \sqcup M_{\tilde{1}} \mathcal{T}} \times \mathcal{S}_{\overline{\mathcal{T}}}(\mu) ; \operatorname{Hom}\left(\pi_{1}^{*} L_{\widetilde{1}}^{*} \otimes \pi_{2}^{*} L_{h}^{\otimes 2}, \pi_{1}^{*} L_{\widehat{1}}^{*} \otimes \mathcal{O}_{1}\right)\right), \\
\alpha_{2}(\widetilde{v})=\pi_{\bar{\nu}_{1}}^{\perp} \circ\left\{\mathcal{D}_{\overline{\mathcal{T}}, h}^{(2)} \widetilde{v}\right\} .
\end{gathered}
$$

By dimension-counting, $\left|M_{\tilde{1}} \mathcal{T}\right| \in\{1,2\}$. If $\left|M_{\tilde{1}} \mathcal{T}\right|=2$, ie $M_{\tilde{1}} \mathcal{T}=\{\widehat{2}, l\}$ for some $l \in[N], \mathcal{S}_{\overline{\mathcal{T}}}(\mu)$ is a finite set and $\mathcal{D}_{\overline{\mathcal{T}}, h}^{(2)}$ does not vanish. Thus, by Propositions 2.18A and 2.18B

$$
\sum_{\chi_{\mathcal{T}}(\tilde{1}, \hat{1})=0,\left|M_{\tilde{1}} \mathcal{T}\right|=2} \mathcal{C}_{\mathcal{U}_{\mathcal{T} \mid \mathcal{T}_{1}}}\left(\alpha_{1}^{\perp}\right)=2\left\langle c_{1}\left(L_{\widetilde{1}}^{*}\right), \overline{\mathfrak{M}}_{\{\tilde{1}, h\} \sqcup M_{\tilde{1}} \mathcal{T}}\right\rangle\left|\mathcal{S}_{1 ; 1}(\mu)\right|=2\left|\mathcal{S}_{1 ; 1}(\mu)\right| .
$$

If $\left|M_{\tilde{1}} \mathcal{T}\right|=1$, that is, $M_{\tilde{1}} \mathcal{T}=\{\widehat{2}\}$, by Propositions $2.18 \mathrm{~A}$ and $2.18 \mathrm{~B}$ and Lemma 4.10.

$$
N\left(\alpha_{2}\right)=\left\langle 4 a_{\widehat{0}}+2 c_{1}\left(\mathcal{L}_{\widetilde{1}}^{*}\right), \overline{\mathcal{S}}_{1}(\mu)\right\rangle-\left|\mathcal{S}_{2}(\mu)\right| .
$$

Thus, summing up the above contributions, we obtain

$$
\sum_{\chi_{\mathcal{T}}(\tilde{1}, \widehat{1})=0,\left|\chi_{\tilde{1}}(\mathcal{T})\right|=1} \mathcal{C}_{\mathcal{U}_{\mathcal{T}_{1}, \mathcal{T}}}\left(\alpha_{1}^{\perp}\right)=\left\langle 8 a_{\widehat{0}}+4 c_{1}\left(\mathcal{L}_{\widetilde{1}}^{*}\right), \overline{\mathcal{S}}_{1}(\mu)\right\rangle+2\left|\mathcal{S}_{1 ; 1}(\mu)\right|-2\left|\mathcal{S}_{2}(\mu)\right| .
$$

(4) Finally, if $\mathcal{T}<\mathcal{T}_{1}, \chi_{\mathcal{T}}(\widetilde{1}, \widehat{1})=0$, and $\chi_{\widetilde{1}}(\mathcal{T})=\left\{h_{1}, h_{2}\right\}$ is a two-element set, the section $\mathcal{D}_{\mathcal{T}, h_{1}}^{(1)}$ does not vanish over the set $\mathcal{S}_{\mathcal{T} \mid \mathcal{T}_{1}}(\mu)$. We denote by

$$
\pi_{h_{1}}: \operatorname{ev}_{\widehat{0}}^{*} T \mathbb{P}^{3} \longrightarrow \operatorname{Im} \mathcal{D}_{\mathcal{T}, h_{1}}^{(1)} \quad \text { and } \quad \pi_{h_{1}}^{\perp}: \operatorname{ev}_{\widehat{0}}^{*} T \mathbb{P}^{3} \longrightarrow E_{h_{1}}
$$

the orthogonal projections, defined over a neighborhood of $\mathcal{S}_{\mathcal{T} \mid \mathcal{T}_{1}}(\mu)$ in $\mathcal{U}_{\mathcal{T}_{1}, \mathcal{T}}$, onto $\operatorname{Im} \mathcal{D}_{\mathcal{T}, h_{1}}^{(1)}$ and its orthogonal complement $E_{1}$ in $\operatorname{ev}_{\widehat{0}}^{*} T \mathbb{P}^{3}$. With appropriate identifications, $\mathcal{S}_{\mathcal{T} \mid \mathcal{T}_{1}}(\mu)$ is the zero set of the section $\operatorname{ev}_{\mathcal{T}_{1}, M_{0}} \oplus \pi_{h_{1}}^{\perp} \circ \mathcal{D}_{\mathcal{T}, h_{2}}^{(1)}$ of the bundle

$$
\mathrm{ev}_{\mathcal{T}_{1}, M_{0}}^{*} \mathcal{N} \Delta_{\mathcal{T}_{1}}(\mu) \oplus L_{h_{2}}^{*} \otimes E_{h_{1}}
$$

defined over a neighborhood of $\mathcal{S}_{\mathcal{T} \mid \mathcal{T}_{1}}(\mu)$ in $\mathcal{U}_{\mathcal{T}_{1}, \mathcal{T}}$. By Lemma 3.4 this section 
is transverse to the zero set. By Proposition 3.5 and Lemma 4.2 .

$$
\begin{gathered}
\operatorname{ev}_{\mathcal{T}_{1}, M_{0}}\left(\phi_{\mathcal{T}_{1}, \mathcal{T}}(b ; v)\right)=\operatorname{ev}_{\mathcal{T}_{1}, M_{0}}(b)+\varepsilon_{-; 1}(b ; v), \\
\pi_{h_{1}}^{\perp}\left\{\operatorname{ev}_{\tilde{1}} \times \operatorname{ev}_{\widehat{1}}\right\}\left(\phi_{\mathcal{T}_{1}, \mathcal{T}}(v)\right)=\left(y_{h_{2} ; \hat{1}}-x_{\widehat{1} ; h_{2}}\right)^{-1} \otimes \\
\left\{\pi_{h_{1}}^{\perp} \circ \mathcal{D}_{\mathcal{T}, h_{2}}^{(1)}+\varepsilon_{-; 2}(v)\right\} \rho_{\mathcal{T}, \widehat{1} ; h_{2}}^{(0 ; 1)}\left(v_{h_{2}}\right), \\
\pi_{h_{1}}\left\{\operatorname{ev}_{\widetilde{1}} \times \operatorname{ev}_{\widehat{1}}\right\}\left(\phi_{\mathcal{T}_{1}, \mathcal{T}}(v)\right)=\left\{y_{h_{1} ; \hat{1}}-x_{\widehat{1} ; h_{1}}\right)^{-1} \otimes\left(\mathcal{D}_{\mathcal{T}, h_{1}}^{(1)}+\varepsilon_{+; h_{1}}(v)\right\} \rho_{\mathcal{T}, \hat{1} ; h_{1}}^{(0 ; 1)}\left(v_{h_{1}}\right) \\
+\left(y_{h_{2} ; \hat{1}}-x_{\widehat{1} ; h_{2}}\right)^{-1} \otimes\left\{\pi_{h_{1}} \mathcal{D}_{\mathcal{T}, h_{2}}^{(1)}+\varepsilon_{+; h_{2}}(v)\right\} \rho_{\mathcal{T}, \widehat{1} ; h_{2}}^{(0 ; 1)}\left(v_{h_{2}}\right)
\end{gathered}
$$

for all $(b ; v) \in \mathcal{F} \mathcal{T}_{\delta}-Y\left(\mathcal{F} \mathcal{T} ; \widehat{I}^{+}\right)$and some $C^{1}$-negligible maps

$$
\begin{aligned}
\varepsilon_{-; 1}, \varepsilon_{-; 2}, \varepsilon_{+; h}: \mathcal{F} \mathcal{T}_{\delta} & -Y\left(\mathcal{F} \mathcal{T} ; \widehat{I}^{+}\right) \\
& \longrightarrow \operatorname{ev}_{\mathcal{T}_{1}, M_{0}}^{*} \mathcal{N} \Delta_{\mathcal{T}_{1}}(\mu), L_{h_{2}}^{*} \otimes E_{h_{1}}, L_{h}^{*} \otimes \operatorname{Im} \mathcal{D}_{\mathcal{T}, h_{1}}^{(1)} .
\end{aligned}
$$

However, subtracting the expansion of $\left\{\mathrm{ev}_{\tilde{1}} \times \mathrm{ev}_{\hat{1}}\right\} \circ \phi_{\mathcal{T}_{1}, \mathcal{T}}$ in Lemma 4.2 multiplied by $\left(y_{\widetilde{1} ; \hat{1}}(v)-x_{\widetilde{1} ; h_{1}}(v)\right)$ from the expansion of $\mathcal{D}_{\mathcal{T}, \widehat{1}}^{(1)} \circ \phi_{\mathcal{T}_{1}, \mathcal{T}}$ in $(3 \mathrm{~b})$ of Proposition 3.5 we find that

$$
\mathcal{D}_{\mathcal{T}_{1}, \widetilde{1}}^{(1)} \phi_{\mathcal{T}_{1}, \mathcal{T}}(v)=\left\{\alpha_{2}^{\prime}+\varepsilon(v)\right\} \rho(v)
$$

for all $v \in \mathcal{F} \mathcal{T}_{\delta}$ such that $\phi_{\mathcal{T}_{1}, \mathcal{T}}(v) \in \mathcal{U}_{\mathcal{T}_{1}}^{(1)}(\mu)$, where $\rho$ is a monomials map on $\mathcal{F} \mathcal{T}$ with values in a rank-two bundle $\widetilde{\mathcal{F}} \mathcal{T}$,

$$
\alpha_{2}^{\prime}: \tilde{\mathcal{F}} \mathcal{T} \longrightarrow L_{\widetilde{1}}^{*} \otimes \mathrm{ev}_{\widehat{0}}^{*} T \mathbb{P}^{3}
$$

is a linear map, such that $\alpha_{+} \oplus \alpha$ is injective over $\mathcal{U}_{\mathcal{T}_{1}}^{(1)}(\mu)$, and

$$
\varepsilon: \mathcal{F} \mathcal{T}-Y\left(\mathcal{F} \mathcal{T} ; \widehat{I}^{+}\right) \longrightarrow \operatorname{Hom}\left(\widetilde{\mathcal{F}} \mathcal{T}, L_{\widetilde{1}}^{*} \otimes \operatorname{ev}_{\widehat{0}}^{*} T \mathbb{P}^{3}\right)
$$

is a $C^{0}$-negligible map. Explicitly, if $\widehat{I}=\chi_{\widetilde{1}}(\mathcal{T}), \rho$ is the identity map, and

$$
\alpha\left(v_{h_{1}}, v_{h_{2}}\right)=\left(y_{\widehat{1}}-x_{h_{1}}\right)^{-1} \otimes\left(x_{h_{2}}-x_{h_{2}}\right) \otimes \mathcal{D}_{\mathcal{T}, h_{2}}^{(1)} v_{h_{2}} .
$$

Thus, if $\bar{\nu}_{1}$ is generic, $\mathcal{S}_{\mathcal{T} \mid \mathcal{T}_{1}}(\mu)$ is $\alpha_{1}^{\perp}$-hollow unless $\widehat{I}^{+}=\chi_{\widetilde{1}}(\mathcal{T})$. If $\widehat{I}^{+}=$ $\chi_{\tilde{1}}(\mathcal{T})$, by dimension-counting $M_{\widetilde{1}} \mathcal{T}=\{\widehat{2}\}$ if $\mathcal{S}_{\mathcal{T} \mid \mathcal{T}_{1}}(\mu) \neq \emptyset$, and by Proposition 2.18B rescaling of the linear map, and the decomposition (3.4),

$$
\begin{gathered}
\mathcal{C}_{\mathcal{S}_{\mathcal{T} \mid \mathcal{T}_{1}}(\mu)}\left(\alpha_{1}^{\perp}\right)=N\left(\alpha_{2}\right), \quad \text { where } \\
\alpha_{2} \in \Gamma\left(\overline{\mathfrak{M}}_{\left\{\widetilde{1}, \widehat{1}, h_{1}, h_{2}\right\}} \times \mathcal{S}_{\overline{\mathcal{T}}}(\mu) ; \operatorname{Hom}\left(\pi_{1}^{*} L_{\widetilde{1}}^{*} \otimes \pi_{2}^{*} L_{h_{2}}, \pi_{1}^{*} L_{\widetilde{1}}^{*} \otimes \mathcal{O}_{1}\right)\right), \\
\alpha_{2}(\widetilde{v})=\pi_{\bar{\nu}_{1}}^{\perp} \circ\left\{\mathcal{D}_{\overline{\mathcal{T}}}^{(1)} v\right\} .
\end{gathered}
$$


The set $\mathcal{S}_{\overline{\mathcal{T}}}(\mu)$ is finite and $\mathcal{D}_{\overline{\mathcal{T}}}^{(1)}$ does not vanish. Thus, by Propositions $2.18 \mathrm{~A}$ and $2.18 \mathrm{~B}$.

$$
\sum_{\chi_{\mathcal{T}}(\tilde{1}, \widehat{1})=0,\left|\chi_{\tilde{1}}(\mathcal{T})\right|=2} \mathcal{C}_{\mathcal{U}_{\mathcal{T} \mid \mathcal{T}_{1}}}\left(\alpha_{1}^{\perp}\right)=\left\langle c_{1}\left(L_{\widetilde{1}}^{*}\right), \overline{\mathfrak{M}}_{\left\{\tilde{1}, \hat{1}, h_{1}, h_{2}\right\}} \mathcal{T}\right\rangle\left|\mathcal{S}_{2}(\mu)\right|=\left|\mathcal{S}_{2}(\mu)\right| .
$$

The claim follows by plugging equations (4.6), (4.7), and (4.8) into (4.5) and using (3.2) and (3.3).

Lemma 4.9 If $\mathcal{O}_{1} \longrightarrow \overline{\mathcal{V}}_{1 ; 1}^{(1)}(\mu)$ is the quotient of the bundle $\operatorname{ev}_{\widehat{0}}^{*} T \mathbb{P}^{3}$ by a generic trivial line subbundle $\mathbb{C} \bar{\nu}_{1}, \pi_{\bar{\nu}_{1}}^{\perp}: \operatorname{ev}_{\widehat{0}}^{*} T \mathbb{P}^{3} \longrightarrow \mathcal{O}_{1}$ is the quotient projection, and $\alpha_{2} \in \Gamma\left(\overline{\mathcal{V}}_{1 ; 1}^{(1)}(\mu) ; \operatorname{Hom}\left(L_{\tilde{1}}, \mathcal{O}_{1}\right)\right)$ is given by $\alpha_{2}=\pi_{\bar{\nu}_{1}}^{\perp} \circ \mathcal{D}_{\mathcal{T}_{1 ; l}, \tilde{1}}^{(1)}$ on $\mathcal{U}_{\mathcal{T}_{1 ; l}}^{(1)}(\mu)$,

$$
N\left(\alpha_{2}\right)=\left\langle 4 a_{\widehat{0}}+c_{1}\left(L_{\widetilde{1}}^{*}\right), \overline{\mathcal{V}}_{1 ; 1}^{(1)}(\mu)\right\rangle-2\left|\mathcal{S}_{1 ; 1}(\mu)\right| .
$$

Proof By Propositions 2.18A and 2.18B

$$
N\left(\alpha_{2}\right)=\left\langle 4 a_{\widehat{0}}+c_{1}\left(L_{\widetilde{1}}^{*}\right), \overline{\mathcal{V}}_{1 ; 1}^{(1)}(\mu)\right\rangle-\mathcal{C}_{\partial \overline{\mathcal{V}}_{1 ; 1}^{(1)}(\mu)}\left(\alpha_{2}^{\perp}\right),
$$

where $\alpha_{2}^{\perp}$ denotes the composition of $\alpha_{2}$ with the projection $\pi_{\bar{\nu}_{2}}^{\perp}$ onto the quotient $\mathcal{O}_{2}$ of $\mathcal{O}_{1}$ by a generic trivial line subbundle $\mathbb{C} \bar{\nu}_{2}$. Suppose

$$
\mathcal{T} \equiv\left(M_{1}, I ; j, \underline{d}\right)<\mathcal{T}_{1 ; l}
$$

is a bubble type such that $\mathcal{D}_{\mathcal{T}_{1 ; l}, \tilde{1}}^{(1)}$ vanishes somewhere on $\overline{\mathcal{V}}_{1 ; 1}^{(1)}(\mu) \cap \mathcal{U}_{\mathcal{T}_{1 ; l}, \mathcal{T}}$. Then, by Lemmas $3.4,4.1$ and 4.2

$$
\mathcal{T}=\mathcal{T}_{1 ; l}(\widehat{1}) \quad \text { and } \quad \overline{\mathcal{V}}_{1 ; 1}^{(1)}(\mu) \cap \mathcal{U}_{\mathcal{T}_{1 ; l}, \mathcal{T}} \subset \mathcal{S}_{\mathcal{T} \mid \mathcal{T}_{1 ; l}}(\mu)
$$

By the same argument as in (3) of the proof of Lemma 4.8.

$$
\mathcal{C}_{\mathcal{S}_{\mathcal{T} \mid \mathcal{T}_{1 ; l}}(\mu)}\left(\alpha_{2}^{\perp}\right)=2\left|\mathcal{S}_{\mathcal{T} \mid \mathcal{T}_{1 ; l}}(\mu)\right| .
$$

We conclude that

$$
\mathcal{C}_{\partial \overline{\mathcal{V}}_{1 ; 1}^{(1)}(\mu)}\left(\alpha_{2}^{\perp}\right)=2\left|\mathcal{S}_{1 ; 1}(\mu)\right| .
$$

The claim follows from (4.9) and (4.10).

Lemma 4.10 If $\mathcal{O}_{1} \longrightarrow \overline{\mathcal{S}}_{1}(\mu)$ is the quotient of the bundle ev ${ }_{\widehat{0}}^{*} T \mathbb{P}^{3}$ by a generic trivial line subbundle $\mathbb{C} \bar{\nu}_{1}, \pi_{\bar{\nu}_{1}}^{\perp}: \operatorname{ev}_{\widehat{0}}^{*} T \mathbb{P}^{3} \longrightarrow \mathcal{O}_{1}$ is the quotient projection, and $\alpha_{2} \in \Gamma\left(\overline{\mathcal{S}}_{1}(\mu) ; \operatorname{Hom}\left(L_{\widetilde{1}}^{\otimes 2}, \mathcal{O}_{1}\right)\right)$ is given by $\alpha_{2}=\pi_{\bar{\nu}_{1}}^{\perp} \circ \mathcal{D}_{\mathcal{T}_{0}, \tilde{1}}^{(2)}$,

$$
N\left(\alpha_{2}\right)=\left\langle 4 a_{\widehat{0}}+2 c_{1}\left(\mathcal{L}_{\widetilde{1}}^{*}\right), \overline{\mathcal{S}}_{1}(\mu)\right\rangle-\left|\mathcal{S}_{2}(\mu)\right|
$$


Proof (1) By Propositions 2.18A and 2.18B

$$
N\left(\alpha_{2}\right)=\left\langle 4 a_{\widehat{0}}+2 c_{1}\left(L_{\widehat{1}}^{*}\right), \overline{\mathcal{S}}_{1}(\mu)\right\rangle-\mathcal{C}_{\partial \overline{\mathcal{S}}_{1}(\mu)}\left(\alpha_{2}^{\perp}\right),
$$

where $\alpha_{2}^{\perp}$ denotes the composition of $\alpha_{2}$ with the projection $\pi_{\bar{\nu}_{2}}^{\perp}$ onto the quotient $\mathcal{O}_{2}$ of $\mathcal{O}_{1}$ by a generic trivial line subbundle $\mathbb{C} \bar{\nu}_{2}$. We now use the expansion (3d) of Proposition 3.5 to describe the boundary strata of $\overline{\mathcal{S}}_{1}(\mu)$ and compute the contribution of each stratum to $\mathcal{C}_{\partial \overline{\mathcal{S}}_{1}(\mu)}\left(\alpha_{2}^{\perp}\right)$. Our description shows that $\partial \overline{\mathcal{S}}_{1}(\mu)$ is a finite set and thus $\mathcal{S}_{1}(\mu)$ is a one-pseudovariety in $\overline{\mathcal{V}}_{1}(\mu)$ and $\overline{\mathcal{V}}_{1}$.

(2) If $\mathcal{T}=\left(M_{0}, I ; j, \underline{d}\right)<\mathcal{T}_{0}$, by Proposition 3.5 ,

$\mathcal{D}_{\mathcal{T}_{0}, \tilde{1}}^{(1)} \phi_{\mathcal{T}_{0}, \mathcal{T}}(v)=\sum_{h \in \chi_{\tilde{1}}(\mathcal{T})}\left(\mathcal{D}_{\mathcal{T}, h}^{(1)}+\varepsilon_{\mathcal{T}, \tilde{1} ; h}^{(1)}\right) \rho_{\mathcal{T}, \widetilde{1}}^{(0 ; 1)}(v), \quad$ where $\quad \rho_{\mathcal{T}, \widetilde{1}}^{(0 ; 1)}(v)=\prod_{i \in(\widetilde{1}, h]} v_{i}$,

for all $v \in \mathcal{F} \mathcal{T}$ sufficiently small. Thus, $\overline{\mathcal{S}}_{1}(\mu) \cap \mathcal{U}_{\mathcal{T}_{0}, \mathcal{T}}$ is contained in the finite set $\mathcal{S}_{\mathcal{T} \mid \mathcal{T}_{0}}(\mu)$. If $d_{\widetilde{1}} \neq 0$, section $\mathcal{D}_{\mathcal{T}_{0}, \tilde{1}}^{(2)}$ does not vanish on $\mathcal{S}_{\mathcal{T} \mid \mathcal{T}_{0}}(\mu)$ and thus $\mathcal{U}_{\mathcal{T} \mid \mathcal{T}_{0}}$ does not contribute to $\mathcal{C}_{\partial \overline{\mathcal{S}}_{1}(\mu)}\left(\alpha_{2}^{\perp}\right)$, since $\mathcal{D}_{\mathcal{T}_{0}, \widetilde{1}}^{(1)}$ is defined everywhere on $\overline{\mathcal{S}}_{1}(\mu)$.

(3) If $d_{\widetilde{1}}=0$ and $\mathcal{S}_{\mathcal{T} \mid \mathcal{T}_{0}}(\mu) \neq \emptyset, \widehat{I}^{+}=\chi_{\widetilde{1}}(\mathcal{T})$ and $\left|\chi_{\widetilde{1}}(\mathcal{T})\right| \in\{1,2\}$. Suppose $\chi_{\tilde{1}}(\mathcal{T})=\{h\}$ is a single-element set, ie $\mathcal{T}=\mathcal{T}_{0}(l)$ for some $l \in[N]$. With appropriate identifications, $\mathcal{S}_{\mathcal{T} \mid \mathcal{T}_{0}}(\mu)$ is the zero set of the section $\operatorname{ev}_{\mathcal{T}_{0}, M_{0}} \oplus \mathcal{D}_{\mathcal{T}, h}^{(1)}$ of the bundle

$$
\mathrm{ev}_{\mathcal{T}_{0}, M_{0}}^{*} \mathcal{N} \Delta_{\mathcal{T}_{0}}(\mu) \oplus L_{h}^{*} \otimes \mathrm{ev}_{\widehat{0}}^{*} T \mathbb{P}^{3}
$$

defined over a neighborhood of $\mathcal{S}_{\mathcal{T} \mid \mathcal{T}_{0}}(\mu)$ in $\mathcal{U}_{\mathcal{T}_{0}, \mathcal{T}}$. By Lemma 3.4 this section is transverse to the zero set. By Proposition 3.5 and Lemma 4.2 .

$$
\begin{gathered}
\mathrm{ev}_{\mathcal{T}_{0}, M_{0}}\left(\phi_{\mathcal{T}_{0}, \mathcal{T}}(b ; v)\right)=\operatorname{ev}_{\mathcal{T}_{0}, M_{0}}(b)+\varepsilon_{-; 1}(b ; v), \\
\mathcal{D}_{\mathcal{T}_{0}, \tilde{1}}^{(1)}\left(\phi_{\mathcal{T}_{0}, \mathcal{T}}(v)\right)=\left\{\mathcal{D}_{\mathcal{T}, h}^{(1)}+\varepsilon_{-; 2}(v)\right\} v,
\end{gathered}
$$

for all $(b ; v) \in \mathcal{F} \mathcal{T}_{\delta}-Y\left(\mathcal{F} \mathcal{T} ; \widehat{I}^{+}\right)$and some $C^{1}$-negligible maps

$$
\varepsilon_{-; 1}, \varepsilon_{-; 2}: \mathcal{F} \mathcal{T}_{\delta}-Y\left(\mathcal{F} \mathcal{T} ; \widehat{I}^{+}\right) \longrightarrow \operatorname{ev}_{\mathcal{T}_{0}, M_{0}}^{*} \mathcal{N} \Delta_{\mathcal{T}_{0}}(\mu), L_{h}^{*} \otimes \mathrm{ev}_{\widehat{0}}^{*} T \mathbb{P}^{3}
$$

On the other hand, subtracting the expansion of $\mathcal{D}_{\mathcal{T}_{0}, \widetilde{1}^{\circ}}^{(1)} \phi_{\mathcal{T}_{0}, \mathcal{T}}$ of $(3 \mathrm{~d})$ of Proposition 3.5 times $x_{h}$ from the expansion of $\mathcal{D}_{\mathcal{T}_{0}, \tilde{1}}^{(2)} \circ \phi_{\mathcal{T}_{0}, \mathcal{T}}$ of $(3 \mathrm{~d})$ of Proposition 3.5. we obtain

$\mathcal{D}_{\mathcal{T}_{0}, \widetilde{1}}^{(2)} \phi_{\mathcal{T}_{0}, \mathcal{T}}(v)=\left\{\mathcal{D}_{\mathcal{T}, h}^{(2)}+\varepsilon_{\mathcal{T}, \widetilde{1} ; h}^{(2)}(v)\right\} v \otimes v \quad$ for all $v \in \mathcal{F} \mathcal{T}_{\delta}$ with $\phi_{\mathcal{T}_{0}, \mathcal{T}}(v) \in \mathcal{S}_{1}$. 
By Lemma 3.4 $\mathcal{D}_{\mathcal{T}, h}^{(2)}$ does not vanish on the finite set $\mathcal{S}_{\mathcal{T} \mid \mathcal{T}_{0}}(\mu)$. Thus, by Proposition 2.18B

$$
\sum_{\left|\chi_{\tilde{1}}(\mathcal{T})\right|=1} \mathcal{C}_{\mathcal{U}_{\mathcal{T}_{0}, \mathcal{T}}(\mu)}\left(\alpha_{2}^{\perp}\right)=2\left|\mathcal{S}_{1 ; 1}(\mu)\right|
$$

(4) If $\chi_{\widetilde{1}}(\mathcal{T})=\left\{h_{1}, h_{2}\right\}$ is a two-element set, the section $\mathcal{D}_{\mathcal{T}, h_{1}}^{(1)}$ does not vanish over the set $\mathcal{S}_{\mathcal{T} \mid \mathcal{T}_{0}}(\mu)$. Let $\pi_{h_{1}}, \pi_{h_{1}}^{\perp}$, and $E_{h_{1}}$ be as in (4) of the proof of Lemma 4.8 Similarly to the previous case, $\mathcal{S}_{\mathcal{T} \mid \mathcal{T}_{0}}(\mu)$ is the zero set of the section

$$
\operatorname{ev}_{\mathcal{T}_{0}, M_{0}} \oplus \pi_{h_{1}}^{\perp} \circ \mathcal{D}_{\mathcal{T}, h_{2}}^{(1)}
$$

of the bundle $\mathrm{ev}_{\mathcal{T}_{0}, M_{0}}^{*} \mathcal{N} \Delta_{\mathcal{T}_{0}}(\mu) \oplus L_{h_{2}}^{*} \otimes E_{h_{1}}$ defined over a neighborhood of $\mathcal{S}_{\mathcal{T} \mid \mathcal{T}_{0}}(\mu)$ in $\mathcal{U}_{\mathcal{T}_{0}, \mathcal{T}}$. By Lemma [3.4 this section is transverse to the zero set. By Proposition 3.5 and Lemma 4.2

$$
\begin{gathered}
\operatorname{ev} \mathcal{T}_{0}, M_{0} \\
\left.\pi_{h_{1}}^{\perp} \mathcal{D}_{\mathcal{T}_{0}, \tilde{1}}^{(1)}\left(\phi_{\mathcal{T}_{0}, \mathcal{T}}(b ; v)\right)=\operatorname{ev} \mathcal{T}_{0}, M_{0}(b)\right)=\left\{\pi_{h_{1}}^{\perp} \circ \mathcal{D}_{\mathcal{T}, h_{2}}^{(1)}+\varepsilon_{-; 2}(v)\right\} v_{h_{2}}, \\
\left.\pi_{h_{1}} \mathcal{D}_{\mathcal{T}_{0}, \tilde{1}}^{(1)}\left(\phi_{\mathcal{T}_{0}, \mathcal{T}}(v)\right)=\left\{\mathcal{D}_{\mathcal{T}, h_{1}}^{(1)}+\varepsilon_{+; h_{1}}(v)\right\} v_{h_{1}}\right)+\left\{\pi_{h_{1}} \mathcal{D}_{\mathcal{T}, h_{2}}^{(1)}+\varepsilon_{+; h_{2}}(v)\right\} v_{h_{2}}
\end{gathered}
$$

for all $(b ; v) \in \mathcal{F} \mathcal{T}_{\delta}-Y\left(\mathcal{F} \mathcal{T} ; \widehat{I}^{+}\right)$and some $C^{1}$-negligible maps $\varepsilon_{-; 1}, \varepsilon_{-; 2}, \varepsilon_{+; h}$. On the other hand, subtracting the expansion of $\mathcal{D}_{\mathcal{T}, \tilde{1}}^{(1)} \circ \phi_{\mathcal{T}_{0}}, \mathcal{T}$ of (3d) of Proposition 3.5 times $x_{h_{1}}$ from the expansion of $\mathcal{D}_{\mathcal{T}_{0}, \tilde{1}^{\circ}}^{(2)} \circ \phi_{\mathcal{T}_{0}, \mathcal{T}}$ of $(3 \mathrm{~d})$ of Proposition 3.5 we obtain

$$
\mathcal{D}_{\mathcal{T}_{0}, \tilde{1}}^{(2)} \phi_{\mathcal{T}_{0}, \mathcal{T}}(v)=\left(x_{h_{2}}-x_{h_{1}}\right) \otimes\left\{\mathcal{D}_{\mathcal{T}, h_{2}}^{(1)}+\varepsilon(v)\right\} v_{h_{2}}
$$

for all $v \in \mathcal{F}_{\mathcal{T}_{\delta}}$ such that $\phi_{\mathcal{T}_{0}, \mathcal{T}}(v) \in \mathcal{S}_{1}(\mu)$,

where $\varepsilon$ is a $C^{0}$-negligible map. Since $\mathcal{D}_{\mathcal{T}, h}^{(1)}$ does not vanish on $\mathcal{S}_{\mathcal{T} \mid \mathcal{T}_{0}}(\mu)$, from Proposition 2.18B, we conclude that

$$
\sum_{\left|\chi_{\tilde{1}}(\mathcal{T})\right|=2} \mathcal{C}_{\mathcal{U}_{\mathcal{T} \mid \mathcal{T}_{0}}(\mu)}\left(\alpha_{2}^{\perp}\right)=\left|\mathcal{S}_{2}(\mu)\right| .
$$

The claim follows by plugging equations (4.12) and (4.13) into (4.11) and using (3.2) and (3.3).

Lemma 4.11 Suppose $\mathcal{T}=\left(M_{2}, I ; j, \underline{d}\right)$ is a bubble type such that $\mathcal{T}<\mathcal{T}_{2}$, $\chi_{\mathcal{T}}(\widetilde{1}, \widehat{2})>0$, and $\chi_{\mathcal{T}}(\widehat{1}, \widehat{2})=0$.

(1) If $\left|\widehat{I}^{+}\right| \neq 1$ or $\left|M_{j_{\hat{1}}} \mathcal{T}\right| \neq 1, \mathcal{C}_{\mathcal{U}_{\mathcal{T}_{2}, \mathcal{T}}}\left(\mathrm{ev}_{\widetilde{1}} \times \mathrm{ev}_{\widehat{2}} ; \Delta_{\mathbb{P}^{3} \times \mathbb{P}^{3}}\right)=0$. 


$$
\begin{aligned}
& \text { If }\left|\widehat{I}^{+}\right|=1 \text { and }\left|M_{j_{\widehat{1}}} \mathcal{T}\right|=1 \\
& \begin{aligned}
\mathcal{C}_{\mathcal{U}_{\mathcal{T}_{2}, \mathcal{T}}}\left(\operatorname{ev}_{\widetilde{1}} \times \operatorname{ev}_{\widehat{2}} ; \Delta_{\mathbb{P}^{3} \times \mathbb{P}^{3}}\right) & =\left\langle 6 a_{\widehat{0}}^{2}+4 a_{\widehat{0}} c_{1}\left(\mathcal{L}_{\widetilde{1}}^{*}\right)+c_{1}^{2}\left(\mathcal{L}_{\widetilde{1}}^{*}\right), \overline{\mathcal{V}}_{1}^{(1)}(\mu)\right\rangle \\
+ & \left|\mathcal{S}_{2}(\mu)\right|-\left|\mathcal{V}_{2}^{(1)}(\mu)\right|-\left\langle 8 a_{\widehat{0}}^{2}+4 c_{1}\left(\mathcal{L}_{\widetilde{1}}^{*}\right), \overline{\mathcal{S}}_{1}(\mu)\right\rangle .
\end{aligned}
\end{aligned}
$$

Proof This lemma follows from Lemma 4.7 by symmetry.

Remark These contributions can be computed directly, ie similarly to the proof of Lemma 4.7 and in fact one finds a somewhat different expression for the contribution in (2). What this means is that we have found a relationship between certain intersection numbers:

$$
\left\langle 8 a_{\widehat{0}} c_{1}\left(\mathcal{L}_{\widetilde{1}}^{*}\right), \overline{\mathcal{V}}_{1}^{(1)}(\mu)\right\rangle+2\left|\mathcal{V}_{2}^{(1)}(\mu)\right|=\left\langle 4 a_{\widehat{0}}-\eta_{\widehat{0}, 1}, \overline{\mathcal{V}}_{2}^{(1,1)}(\mu)\right\rangle+\left|\mathcal{V}_{2,(0,1)}^{(1 ; 0,1)}(\mu)\right|
$$

Here $\mathcal{V}_{2,(0,1)}^{(1 ; 0,1)}(\mu)$ denotes a set of tuples of stable maps whose cardinality is six times the number of rational curves that pass through the constraints $\mu$ and have the form described by the last picture of Figure 1. Using Lemma 2.2.2 in [11, it is possible to restate the relation (4.14) in terms of numbers of rational curves of various shapes.

Lemma 4.12 If $\mathcal{T}=\left(M_{2}, I ; j, \underline{d}\right)$ is a bubble type such that $\mathcal{T}<\mathcal{T}_{2}$ and $\chi_{\mathcal{T}}(\widetilde{1}, \widehat{2})=\chi_{\mathcal{T}}(\widehat{1}, \widehat{2})=0$,

$$
\mathcal{C}_{\mathcal{U}_{\mathcal{T}_{2}, \mathcal{T}}}\left(\mathrm{ev}_{\widetilde{1}} \times \mathrm{ev}_{\widehat{2}} ; \Delta_{\mathbb{P}^{3} \times \mathbb{P}^{3}}\right)=0 .
$$

Proof (1) Since $\chi_{\mathcal{T}}(\widetilde{1}, \widehat{1})=0$, by Lemma 4.2

$$
\overline{\mathcal{U}}_{\mathcal{T}_{2}}^{(1)}(\mu) \cap \mathcal{U}_{\mathcal{T}_{2}, \mathcal{T}} \subset \mathcal{S}_{\mathcal{T} \mid \mathcal{T}_{2}}(\mu)
$$

If $\mathcal{S}_{\mathcal{T} \mid \mathcal{T}_{2}}(\mu) \neq \emptyset,\left|\chi_{\widetilde{1}}(\mathcal{T})\right| \in\{1,2\}$. If $h$ is the unique element of $\chi_{\widetilde{1}}(\mathcal{T})$, mixing the argument in (1) of the proof of Lemma 4.7 with (3) of the proof of Lemma 4.8, we find that $\mathcal{S}_{\mathcal{T} \mid \mathcal{T}_{2}}(\mu)$ is $\left(\operatorname{ev}_{\widetilde{1}} \times \mathrm{ev}_{\widehat{2}}, \Delta_{\mathbb{P}^{3} \times \mathbb{P}^{3}}\right)$-hollow unless $\widehat{I}^{+}=$ $\chi_{\widetilde{1}}(\mathcal{T})$. On the other hand, if $\widehat{I}^{+}=\chi_{\widetilde{1}}(\mathcal{T})=\{h\}$, by Proposition 2.18B the decomposition (3.4), and a rescaling of the linear map,

$$
\begin{gathered}
\mathcal{C}_{\mathcal{U}_{\mathcal{T}_{2}, \mathcal{T}}}\left(\mathrm{ev}_{\widetilde{1}} \times \mathrm{ev}_{\widehat{2}} ; \Delta_{\mathbb{P}^{3} \times \mathbb{P}^{3}}\right)=2 N\left(\alpha_{1}\right), \quad \text { where } \\
\alpha_{1}=\pi_{2}^{*} \mathcal{D}_{\overline{\mathcal{T}}}^{(2)} \in \Gamma\left(\overline{\mathfrak{M}}_{\{\widetilde{1}, h\} \sqcup M_{\tilde{1}} \mathcal{T}} \times \mathcal{S}_{\overline{\mathcal{T}}}(\mu) ; \pi_{2}^{*} \operatorname{Hom}\left(L_{\widetilde{1}}^{*}, \operatorname{ev}_{\widehat{0}}^{*} T \mathbb{P}^{3}\right)\right) .
\end{gathered}
$$

Since $\left|M_{\tilde{1}} \mathcal{T}\right| \geq 2$, the first factor is positive-dimensional, while the linear map $\alpha_{1}$ comes entirely from the second factor. Thus,

$$
\mathcal{C}_{\mathcal{U}_{\mathcal{T}_{2}, \mathcal{T}}}\left(\mathrm{ev}_{\widetilde{1}} \times \mathrm{ev}_{\widehat{2}} ; \Delta_{\mathbb{P}^{3} \times \mathbb{P}^{3}}\right)=0 .
$$




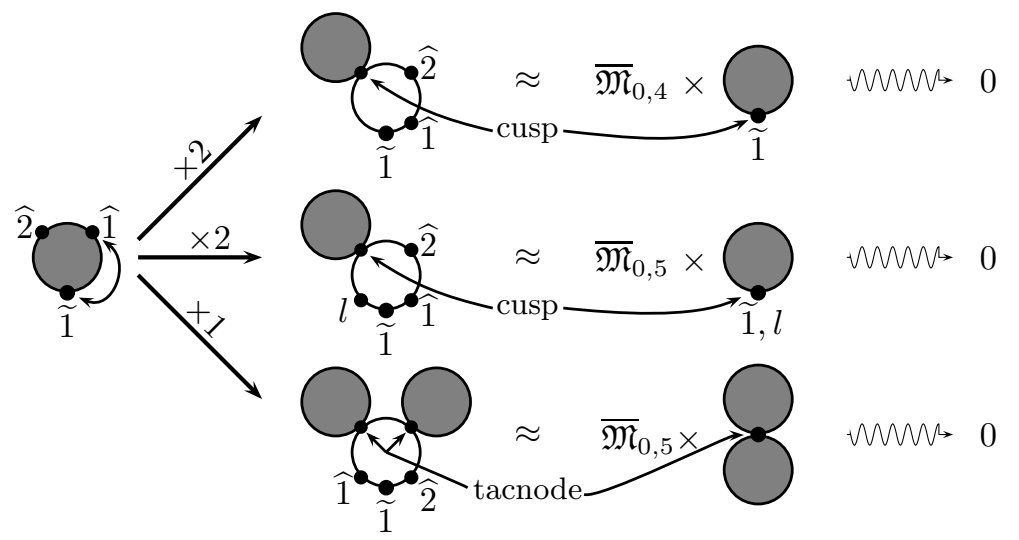

Figure 11: An outline of the proof of Lemma 4.12

(2) If $\chi_{\widetilde{1}}(\mathcal{T})=\left\{h_{1}, h_{2}\right\}$ is a two-element set, the section $\mathcal{D}_{\mathcal{T}, h_{1}}^{(1)}$ does not vanish over the set $\mathcal{S}_{\mathcal{T} \mid \mathcal{T}_{2}}(\mu)$. Mixing (1) of the proof of Lemma 4.7 with (4) of the proof of Lemma 4.8, we find that $\mathcal{S}_{\mathcal{T} \mid \mathcal{T}_{2}}(\mu)$ is $\left(\mathrm{ev}_{\tilde{1}} \times \mathrm{ev}_{\widehat{2}}, \Delta_{\mathbb{P}^{3} \times \mathbb{P}^{3}}\right)$-hollow unless $\widehat{I}^{+}=$ $\chi_{\tilde{1}}(\mathcal{T})$. On the other hand, if $\widehat{I}^{+}=\chi_{\tilde{1}}(\mathcal{T})=\left\{h_{1}, h_{2}\right\}$, by Proposition 2.18B, the decomposition (3.4), and a rescaling of the linear map,

$$
\begin{gathered}
\mathcal{C}_{\mathcal{U}_{\mathcal{T}_{2}, \mathcal{T}}}\left(\mathrm{ev}_{\widetilde{1}} \times \mathrm{ev}_{\widehat{2}} ; \Delta_{\mathbb{P}^{3} \times \mathbb{P}^{3}}\right)=N\left(\alpha_{1}\right), \quad \text { where } \\
\alpha_{1} \in \Gamma\left(\overline{\mathfrak{M}}_{\left\{\widetilde{1}, h_{1}, h_{2}\right\} \sqcup M_{\tilde{1}} \mathcal{T}} \times \mathcal{S}_{\overline{\mathcal{T}}}(\mu) ; \pi_{2}^{*} \operatorname{Hom}\left(L_{h_{1}} \oplus L_{h_{2}}, \operatorname{Im} \mathcal{D}_{\overline{\mathcal{T}}}^{(1)} \oplus \operatorname{ev}_{\widehat{0}}^{*} T \mathbb{P}^{3}\right)\right), \\
\alpha_{1}\left(v_{h_{1}}, v_{h_{2}}\right)=\left(\mathcal{D}_{\overline{\mathcal{T}}}^{(1)} v_{h_{1}}+\mathcal{D}_{\overline{\mathcal{T}}}^{(1)} v_{h_{2}}, \mathcal{D}_{\overline{\mathcal{T}}}^{(1)} v_{h_{2}}\right) .
\end{gathered}
$$

Since $\left|M_{\tilde{1}} \mathcal{T}\right| \geq 2$, the first factor is positive-dimensional, while the linear map $\alpha_{1}$ comes entirely from the second factor. Thus,

$$
\mathcal{C}_{\mathcal{U}_{\mathcal{T}_{2}, \mathcal{T}}}\left(\mathrm{ev}_{\widetilde{1}} \times \mathrm{ev}_{\widehat{2}} ; \Delta_{\mathbb{P}^{3} \times \mathbb{P}^{3}}\right)=0 .
$$

Corollary 4.13 The contribution from the boundary to the number $\left\langle\left\langle\mathcal{V}_{1}^{(2)}(\mu)\right\rangle\right\rangle$ is given by

$$
\begin{array}{r}
\mathcal{C}_{\partial \overline{\mathcal{U}}_{\mathcal{T}_{2}}^{(2)}}\left(\mathrm{ev}_{\widetilde{1}} \times \mathrm{ev}_{\widehat{2}} ; \Delta_{\mathbb{P}^{3} \times \mathbb{P}^{3}}\right)=\left\langle 12 a_{\widehat{0}}^{2}+8 a_{\widehat{0}} \eta_{\widehat{0}, 1}+2 \eta_{\widehat{0}, 1}^{2}, \overline{\mathcal{V}}_{1}^{(1)}(\mu)\right\rangle+2\left|\mathcal{S}_{2}(\mu)\right| \\
-2\left|\mathcal{V}_{2}^{(1)}(\mu)\right|-\left\langle 16 a_{\widehat{0}}+8 \eta_{\widehat{0}, 1}, \overline{\mathcal{S}}_{1}(\mu)\right\rangle .
\end{array}
$$

Proof This corollary follows immediately from Lemmas 4.14 4.4, 4.7, 4.11, and 4.12 . 


\section{Example 2: Rational tacnodal curves in $\mathbb{P}^{3}$}

\subsection{Summary}

In this section, we prove Theorem 1.3. The general approach is the same as in Section 4. If $\mathcal{T}_{1}$ is the bubble type as defined in Section 4, let

$$
\begin{gathered}
\mathcal{S}_{1}^{(1)}(\mu)=\left\{\left(b,\left[v_{\widetilde{1}}, v_{\widetilde{1}}\right]\right) \in \mathbb{P}\left(L_{\widetilde{1}} \oplus L_{\widetilde{1}}^{*}\right) \rightarrow \overline{\mathcal{V}}_{1}^{(1)}: b \in \mathcal{V}_{1}^{(1)}(\mu), \mathcal{D}_{\widetilde{1}, \widehat{1}}\left(b,\left[v_{\widetilde{1}}, v_{\widehat{1}}\right]\right)=0\right\} \\
\text { where } \quad \mathcal{D}_{\widetilde{1}, \widehat{1}} \in \Gamma\left(\mathbb{P}\left(L_{\widetilde{1}} \oplus L_{\widetilde{1}}^{*}\right) \mid \mathcal{V}_{1}^{(1)}(\mu) ; \gamma_{L_{\tilde{1}} \oplus L_{\widetilde{1}}^{*}}^{*} \otimes \operatorname{ev}_{\widehat{0}}^{*} T \mathbb{P}^{3}\right), \\
\mathcal{D}_{\widetilde{1}, \widehat{1}}\left(v_{\widetilde{1}}, v_{\widehat{1}}\right)=\mathcal{D}_{\mathcal{T}_{1}, \widetilde{1}_{\tilde{1}}}^{(1)} v_{\widetilde{1}}+\mathcal{D}_{\mathcal{T}_{1}, \widehat{1}_{1}}^{(1)} v_{\widehat{1}} .
\end{gathered}
$$

The set $\mathcal{S}_{1}^{(1)}(\mu)$ can be identified with the set of rational one-component tacnodal curves passing through the constraints $\mu$, but with a choice of a branch at each node. In particular, the cardinality of the set $\mathcal{S}_{1}^{(1)}(\mu)$ is twice the enumerative number of Theorem 1.3 .

Note that section $\mathcal{D}_{\widetilde{1}, \hat{1}}$ does not extend continuously over all of the boundary of $\overline{\mathcal{V}}_{1}^{(1)}(\mu)$. In fact, this can be seen from (3c) of Proposition 3.5. Nevertheless, the behavior of this section can be understood everywhere. By Proposition 2.18B and equation (2.16), we have

$$
\left|\mathcal{S}_{1}^{(1)}(\mu)\right|=\left\langle 6 a_{\widehat{0}}^{2}+c_{1}^{2}\left(L_{\widetilde{1}}^{*}\right), \overline{\mathcal{V}}_{1}^{(1)}(\mu)\right\rangle-\mathcal{C}_{\partial \mathbb{P}\left(L_{\tilde{1}} \oplus L_{\hat{1}}^{*}\right)}\left(\mathcal{D}_{\tilde{1}, \hat{1}}\right),
$$

where $\mathcal{C}_{\partial \mathbb{P}\left(L_{\tilde{1}} \oplus L_{\tilde{1}}^{*}\right)}\left(\mathcal{D}_{\widetilde{1}, \hat{1}}\right)$ is the $\mathcal{D}_{\widetilde{1}, \hat{1}}$-contribution from the boundary strata of $\mathbb{P}\left(L_{\widetilde{1}} \oplus L_{\widetilde{1}}^{*}\right)$ to the euler class of $\gamma_{L_{\tilde{1}} \oplus L_{\widetilde{1}}^{*}}^{*} \otimes \mathrm{ev}_{\widehat{0}}^{*} T \mathbb{P}^{3}$. This contribution is computed in the rest of this section. Theorem 1.3 is obtained by plugging the expressions of Corollaries 5.3 and 5.9 into (5.1) and then using identities (3.2) and (3.3).

Before proceeding with our computation of the contributions from various strata, we observe that the section $\mathcal{D}_{\widetilde{1}, \hat{1}}$ extends over $\overline{\mathcal{V}}_{1}^{(1)}(\mu) \cap \mathcal{U}_{\mathcal{T}_{1}, \mathcal{T}}$ if

$$
\mathcal{T} \equiv\left(M_{1}, I ; j, \underline{d}\right)<\mathcal{T}_{1}
$$

is a bubble type such that $j_{\widehat{1}}=\widetilde{1}$, as can be seen from Proposition 3.5. If in addition $d_{\tilde{1}} \neq 0$, by Lemmas 3.4 and 4.1. $\mathcal{D}_{\tilde{1}, \hat{1}}$ does not vanish over

$$
\overline{\mathcal{V}}_{1}^{(1)}(\mu) \cap \mathcal{U}_{\mathcal{T}_{1}, \mathcal{T}} \subset \mathcal{U}_{\mathcal{T} \mid \mathcal{T}_{1}}^{(1)}(\mu) .
$$

Thus, such spaces $\mathcal{U}_{\mathcal{T}_{1}, \mathcal{T}}$ do not contribute to $\mathcal{C}_{\partial \mathbb{P}\left(L_{\widetilde{1}} \oplus L_{\widetilde{1}}^{*}\right)}\left(\mathcal{D}_{\tilde{1}, \widehat{1}}\right)$ and will not be considered below. 


\subsection{Contributions from the spaces $\mathcal{U}_{\mathcal{T}_{1}, \mathcal{T}}$ with $\chi_{\mathcal{T}}(\widetilde{1}, \widehat{1})=0$}

In this subsection, we prove Corollary 5.3. which gives the total contribution to $\mathcal{C}_{\partial \mathbb{P}\left(L_{\widetilde{1}} \oplus L_{\widetilde{1}}^{*}\right)}\left(\mathcal{D}_{\widetilde{1}, \hat{1}}\right)$ from all the spaces $\mathcal{U}_{\mathcal{T}_{1}, \mathcal{T}}$, where

$$
\mathcal{T} \equiv\left(M_{1}, I ; j, \underline{d}\right)<\mathcal{T}_{1}
$$

is a bubble type such that $\chi_{\mathcal{T}}(\widetilde{1}, \widehat{1})=0$. We use Lemma 4.2, which describes the intersection $\overline{\mathcal{V}}_{1}^{(1)}(\mu) \cap \mathcal{U}_{\mathcal{T}_{1}, \mathcal{T}}$, along with Proposition 3.5.

Figure 12 shows the three types of boundary strata $\overline{\mathcal{V}}_{1}^{(1)}(\mu) \cap \mathcal{U}_{\mathcal{T}_{1}, \mathcal{T}}$ such that

$$
\mathbb{P}\left(L_{\tilde{1}} \oplus L_{\widetilde{1}}^{*}\right) \mid \overline{\mathcal{V}}_{1}^{(1)}(\mu) \cap \mathcal{U}_{\mathcal{T}_{1}, \mathcal{T}}
$$

is not contained in a finite union of $\mathcal{D}_{\widetilde{1}, \hat{1}}-$ hollow sets. For such boundary strata,

$$
\mathbb{P}\left(L_{\tilde{1}} \oplus L_{\widetilde{1}}^{*}\right) \mid \overline{\mathcal{V}}_{1}^{(1)}(\mu) \cap \mathcal{U}_{\mathcal{T}_{1}, \mathcal{T}}
$$

is a union of two $\mathcal{D}_{\widetilde{1}, \hat{1}}$-regular subsets: a section over the base $\overline{\mathcal{V}}_{1}^{(1)}(\mu) \cap \mathcal{U}_{\mathcal{T}_{1}, \mathcal{T}}$ and its complement. Each number in the odd rows of the last column in Figure 12 gives the multiplicity with which the number $N(\alpha)$ of zeros of an affine map over the larger $\mathcal{D}_{\widetilde{1}, \hat{1}}-$ regular set enters into $\mathcal{C}_{\partial \mathbb{P}\left(L_{\tilde{1}} \oplus L_{\tilde{1}}^{*}\right)}\left(\mathcal{D}_{\widetilde{1}, \widehat{1}}\right)$; each number in the even rows gives such a multiplicity for the smaller set. Lemma $5.1 \mathrm{com}-$ putes the contributions from the first two types of boundary strata of Figure 12 Lemma 5.2 deals with remaining one.

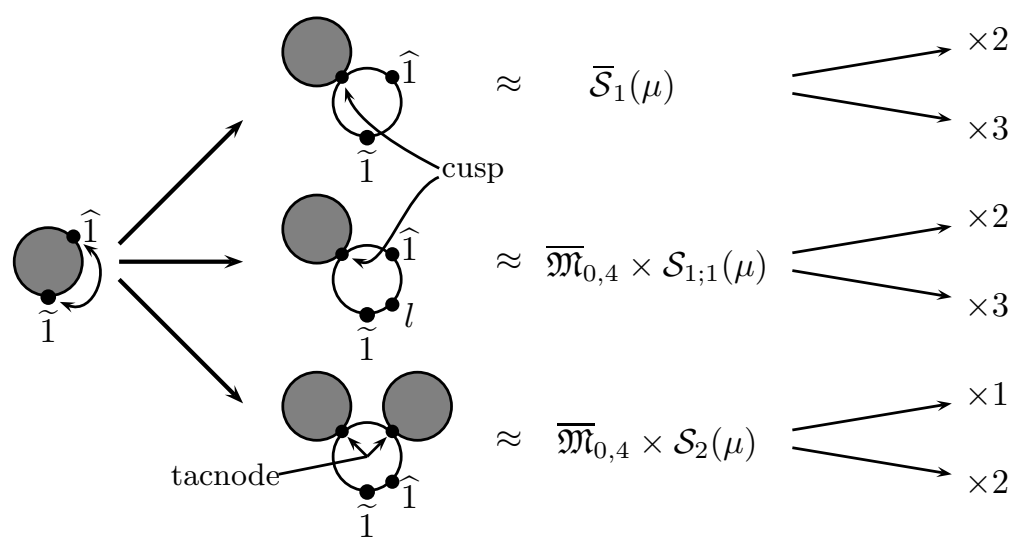

Figure 12: An outline of Subsection 5.2 
Lemma 5.1 Suppose $\mathcal{T}=\left(M_{1}, I ; j, \underline{d}\right)$ is a bubble type such that $\mathcal{T}<\mathcal{T}_{1}$, $\chi_{\mathcal{T}}(\widetilde{1}, \widehat{1})=0$, and $\left|\chi_{\widetilde{1}}(\mathcal{T})\right|=1$.

(1) If $\left|\widehat{I}^{+}\right|>\left|\chi_{\widetilde{1}}(\mathcal{T})\right|, \mathbb{P}\left(L_{\widetilde{1}} \oplus L_{\widetilde{1}}^{*}\right) \mid \mathcal{S}_{\mathcal{T} \mid \mathcal{T}_{1}}(\mu)$ is a finite union of $\mathcal{D}_{\widetilde{1}, \hat{1}}$-hollow subsets and thus

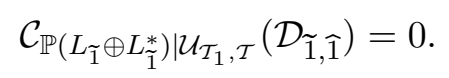

(2) If $\left|\widehat{I}^{+}\right|=\left|\chi_{\widetilde{1}}(\mathcal{T})\right|$ and $M_{\widetilde{1}} \mathcal{T}=\{\widehat{1}\}$,

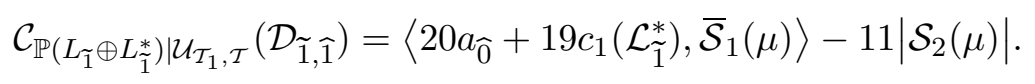

(3) If $\left|\widehat{I}^{+}\right|=\left|\chi_{\widetilde{1}}(\mathcal{T})\right|$ and $M_{\widetilde{1}} \mathcal{T}=\{\widehat{1}, l\}$ for some $l \in[N]$,

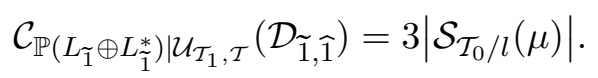

Proof (1) Let $h$ be the unique element of $\chi_{\widetilde{1}}(\mathcal{T})$. By Lemma 4.2

$$
\overline{\mathcal{V}}_{1}^{(1)}(\mu) \cap \mathcal{U}_{\mathcal{T}_{1}, \mathcal{T}} \subset \mathcal{S}_{\mathcal{T} \mid \mathcal{T}_{1}}(\mu) \equiv\left\{b \in \mathcal{U}_{\mathcal{T} \mid \mathcal{T}_{1}}(\mu): \mathcal{D}_{\mathcal{T}, h}^{(1)} b=0\right\} .
$$

With appropriate identifications, $\mathcal{S}_{\mathcal{T} \mid \mathcal{T}_{1}}(\mu)$ is the zero set of the section

$$
\operatorname{ev}_{\mathcal{T}_{1}, M_{0}} \oplus \mathcal{D}_{\mathcal{T}, h}^{(1)}
$$

of the bundle

$$
\mathrm{ev}_{\mathcal{T}_{1}, M_{0}}^{*} \mathcal{N} \Delta_{\mathcal{T}_{1}}(\mu) \oplus L_{h}^{*} \otimes \mathrm{ev}_{\widehat{0}}^{*} T \mathbb{P}^{3}
$$

defined over a neighborhood of $\mathcal{S}_{\mathcal{T} \mid \mathcal{T}_{1}}(\mu)$ in $\mathcal{U}_{\mathcal{T}_{1}, \mathcal{T}}$. By Lemma 3.4 this section is transverse to the zero set. By Proposition 3.5] and Lemma 4.2.

$$
\begin{gathered}
\operatorname{ev}_{\mathcal{T}_{1}, M_{0}}\left(\phi_{\mathcal{T}_{1}, \mathcal{T}}(b ; v)\right)=\operatorname{ev}_{\mathcal{T}_{1}, M_{0}}(b)+\varepsilon_{-; 1}(b ; v), \\
\left\{\operatorname{ev}_{\tilde{1}} \times \operatorname{ev}_{\widehat{1}}\right\}\left(\phi_{\mathcal{T}_{1}, \mathcal{T}}(v)\right)=\left(y_{h ; \hat{1}}-x_{\widehat{1} ; h}\right)^{-1} \otimes\left\{\mathcal{D}_{\mathcal{T}, h}^{(1)}+\varepsilon_{-; 2}(v)\right\} \otimes \rho_{\mathcal{T}, \widehat{1} ; h}^{(0 ; 1)}(v)
\end{gathered}
$$

for all $(b ; v) \in \mathcal{F} \mathcal{T}_{\delta}-Y\left(\mathcal{F} \mathcal{T} ; \widehat{I}^{+}\right)$and some $C^{1}$-negligible maps

$$
\varepsilon_{-; 1}, \varepsilon_{-; 2}: \mathcal{F} \mathcal{T}_{\delta}-Y\left(\mathcal{F} \mathcal{T} ; \widehat{I}^{+}\right) \longrightarrow \mathrm{ev}_{\mathcal{T}_{1}, M_{0}}^{*} \mathcal{N} \Delta_{\mathcal{T}_{2}}(\mu), L_{h}^{*} \otimes \mathrm{ev}_{\widehat{0}}^{*} T \mathbb{P}^{3}
$$

(2) Subtracting the expansion of $\left\{\mathrm{ev}_{\widetilde{1}} \times \mathrm{ev}_{\hat{1}}\right\} \circ \phi_{\mathcal{T}_{1}, \mathcal{T}}$ of Lemma 4.2 multiplied by

$$
\left(y_{\tilde{1} ; \hat{1}}(v)-x_{\widetilde{1} ; h}(v)\right) \quad \text { and by } \quad-\left(y_{\tilde{1} ; \tilde{1}}(v)-x_{\widetilde{1} ; h}(v)\right)^{-1}
$$

from the expansions of $\mathcal{D}_{\mathcal{T}_{1}, \tilde{1}}^{(1)} \circ \phi_{\mathcal{T}_{1}, \mathcal{T}}$ and $\mathcal{D}_{\mathcal{T}_{1}, \widehat{1}}^{(1)} \circ \phi_{\mathcal{T}_{1}, \mathcal{T}}$, respectively, given by Proposition 3.5 we obtain

$$
\mathcal{D}_{\widetilde{1}, \hat{1}} \phi_{\mathcal{T}_{1}, \mathcal{T}}\left(\left[v_{\widetilde{1}}, v_{\widehat{1}}\right] ; v\right)=\{\alpha+\varepsilon(v)\} \rho(v)
$$

for all $\left(\left[v_{\tilde{1}}, v_{\widehat{1}}\right] ; v\right) \in \mathcal{F} \mathcal{T}_{\delta}$ with $\phi_{\mathcal{T}_{1}, \mathcal{T}}(v) \in \mathcal{V}_{1}^{(1)}$, 
where $\rho$ is a monomials map on $\mathcal{F} \mathcal{T}$ with values in a line bundle $\widetilde{\mathcal{F}} \mathcal{T}$,

$$
\alpha: \widetilde{\mathcal{F}} \mathcal{T} \longrightarrow \gamma_{L_{\tilde{1}} \oplus L_{\widehat{1}}^{*}}^{*} \otimes \mathrm{ev}_{\widehat{0}}^{*} T \mathbb{P}^{3}
$$

is a linear map, and

$$
\varepsilon: \mathcal{F} \mathcal{T}-Y\left(\mathcal{F} \mathcal{T} ; \widehat{I}^{+}\right) \longrightarrow \operatorname{Hom}\left(\widetilde{\mathcal{F}} \mathcal{T}, \gamma_{L_{\tilde{1}} \oplus L_{\widetilde{1}}^{*}}^{*} \otimes \mathrm{ev}_{\widehat{0}}^{*} T \mathbb{P}^{3}\right)
$$

is a $C^{0}$-negligible map. Explicitly,

$$
\begin{gathered}
\rho(v)=\prod_{i \in\left(i_{\mathcal{T}}(h, \widehat{1}), h\right]} v_{i}^{\otimes 2} \otimes \prod_{i \in\left(\widetilde{1}, i_{\mathcal{T}}(h, \widehat{1})\right]} v_{i}^{*}, \\
\alpha\left(\left[v_{\widetilde{1}}, v_{\widehat{1}}\right], \widetilde{v}\right)=-\left(y_{h ; \widehat{1}}-x_{\widehat{1} ; h}\right)^{-3} \otimes \mathcal{D}_{\mathcal{T}, h}^{(2)} \widetilde{v} \\
\otimes \begin{cases}\left(y_{h ; \hat{1}}-x_{\widehat{1} ; h}\right)^{2} \otimes v_{\widetilde{1}}+v_{\widehat{1}}, & \text { if } i_{\mathcal{T}}(h, \widehat{1})=\widetilde{1} ; \\
v_{\widehat{1}}, & \text { if } i_{\mathcal{T}}(h, \widehat{1})>\widetilde{1} .\end{cases}
\end{gathered}
$$

In particular, $\alpha$ is an injective linear map outside of a section $\mathcal{Z}_{\mathcal{T}}$ of $\mathbb{P}\left(L_{\widetilde{1}} \oplus L_{\widetilde{1}}^{*}\right)$ over $\mathcal{S}_{\mathcal{T} \mid \mathcal{T}_{1}}(\mu)$. Thus, $\mathbb{P}\left(L_{\widetilde{1}} \oplus L_{\widetilde{1}}^{*}\right) \mid \mathcal{S}_{\mathcal{T} \mid \mathcal{T}_{1}}(\mu)-\mathcal{Z}_{\mathcal{T}}$ is $\mathcal{D}_{\widetilde{1}, \hat{1}}$-hollow unless $\widehat{I}^{+}=$ $\chi_{\widetilde{1}}(\mathcal{T})$. If $\widehat{I}^{+}=\chi_{\widetilde{1}}(\mathcal{T})$, by Proposition 2.18B the decomposition (3.4), and a rescaling of the linear map,

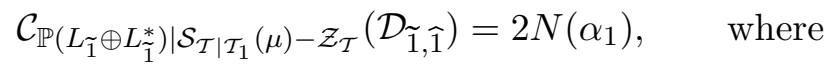

$$
\begin{aligned}
& \alpha_{1} \in \Gamma\left(\mathbb{P} \mathcal{F} \times \mathcal{S}_{\overline{\mathcal{T}}}(\mu) ; \operatorname{Hom}\left(\gamma_{\mathcal{F}}^{*} \otimes L_{h}^{\otimes 2}, \gamma_{\mathcal{F}}^{*} \otimes \mathrm{ev}_{\widehat{0}}^{*} T \mathbb{P}^{3}\right)\right) \text {, } \\
& \mathcal{F}=L_{\tilde{1}} \oplus L_{\widetilde{1}}^{*} \longrightarrow \overline{\mathfrak{M}}_{\{\tilde{1}, h\} \sqcup M_{\tilde{1}} \mathcal{T}}, \quad \alpha_{1}=\mathcal{D}_{\overline{\mathcal{T}}, h}^{(2)} .
\end{aligned}
$$

If $M_{\widetilde{1}} \mathcal{T}=\{\widehat{1}, l\}$ for some $l \in[N], \mathcal{D}_{\overline{\mathcal{T}}, h}^{(2)}$ does not vanish on the finite set $\mathcal{S}_{\overline{\mathcal{T}}}(\mu)$ and thus

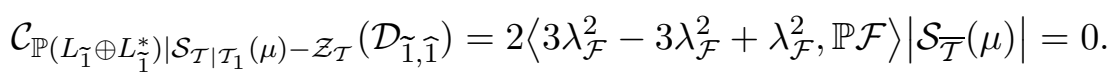

If $M_{\tilde{1}} \mathcal{T}=\{\widehat{1}\}, \overline{\mathcal{T}}=\mathcal{T}_{0}$, and by Propositions 2.18A and 2.18B and identity (2.16),

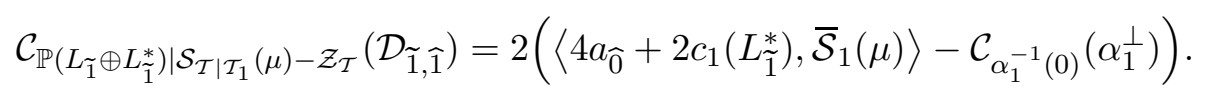

The zero set of $\alpha_{1}$ is precisely $\mathbb{P} \mathcal{F} \times \mathcal{D}_{\mathcal{T}_{0}, \tilde{1}}^{(2)-1}(0)$. From the argument in (3) and (4) of the proof of Lemma 4.10, we obtain

$$
\mathcal{C}_{\alpha_{1}^{-1}(0)}\left(\alpha_{1}^{\perp}\right)=\left\langle\lambda_{\mathcal{F}}, \mathbb{P} \mathcal{F}\right\rangle\left(2\left|\mathcal{S}_{1 ; 1}(\mu)\right|+\left|\mathcal{S}_{2}(\mu)\right|\right)=2\left|\mathcal{S}_{1 ; 1}(\mu)\right|+\left|\mathcal{S}_{2}(\mu)\right| .
$$

Putting the last two equations together, we conclude that if $M_{\tilde{1}} \mathcal{T}=\{\widehat{1}\}$,

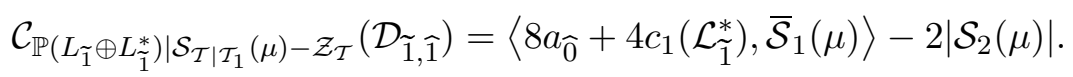


(3) In order to compute the contribution from the space $\mathcal{Z}_{\mathcal{T}}$, we keep the two leading terms of the expression for $\mathcal{D}_{\tilde{1}, \hat{1}}$ obtained as in (2). We can model a neighborhood of $\mathcal{Z}_{\mathcal{T}}$ in $\mathbb{P}\left(L_{\widetilde{1}} \oplus L_{\widetilde{1}}^{*}\right)$ by the map

$$
L_{\widetilde{1}}^{*} \otimes L_{\widetilde{1}}^{*} \longrightarrow \mathbb{P}\left(L_{\widetilde{1}} \oplus L_{\widetilde{1}}^{*}\right), \quad\left(\left[v_{\widetilde{1}}, v_{\widehat{1}}\right], u\right) \longrightarrow\left[v_{\widetilde{1}}, v_{\widehat{1}}+u\left(v_{\widetilde{1}}\right)\right] .
$$

If $i_{\mathcal{T}}(\widehat{1}, h)=\widetilde{1}$, near $\mathcal{Z}_{\mathcal{T}}$,

$$
\begin{aligned}
\mathcal{D}_{\widetilde{1}, \hat{1}} \phi_{\mathcal{T}_{1}, \mathcal{T}}\left(\left[v_{\tilde{1}}, v_{\widehat{1}}\right] ; u, v\right)= & -\left(y_{\widetilde{1} ; \tilde{1}}-x_{\widetilde{1} ; h}\right)^{-3} \otimes\left(\mathcal{D}_{\mathcal{T}, h}^{(2)}+\varepsilon_{2}(u, v)\right) \rho_{\mathcal{T}, \tilde{1} ; h}^{(0 ; 2)}(v) \otimes u \\
& -\left(y_{\widetilde{1} ; \tilde{1}}-x_{\widetilde{1} ; h}\right)^{-2} \otimes v_{\widetilde{1}} \otimes\left(\mathcal{D}_{\mathcal{T}, h}^{(3)}+\varepsilon_{3}(u, v)\right) \rho_{\mathcal{T}, \widetilde{1} ; h}^{(0 ; 3)}(v) .
\end{aligned}
$$

Note that by Lemma 3.4, the images of $\mathcal{D}_{\mathcal{T}, h}^{(2)}$ and $\mathcal{D}_{\mathcal{T}, h}^{(3)}$ are distinct over $\mathcal{S}_{\mathcal{T} \mid \mathcal{T}_{1}}(\mu)$. If $i_{\mathcal{T}}(\widehat{1}, h)>\widetilde{1}$, we similarly find $\mathcal{Z}_{\mathcal{T}}$ is $\mathcal{D}_{\widetilde{1}, \hat{1}}$-hollow unless $\widehat{I}^{+}=$ $\chi_{\widetilde{1}}(\mathcal{T})$. If $\widehat{I}^{+}=\chi_{\widetilde{1}}(\mathcal{T})$, by Proposition 2.18B the decomposition (3.4), and a rescaling of the linear map,

$$
\begin{gathered}
\mathcal{C}_{\mathcal{Z}_{\mathcal{T}}}\left(\mathcal{D}_{\tilde{1}, \hat{1}}\right)=3 N\left(\alpha_{1}\right), \quad \text { where } \\
\alpha_{1} \in \Gamma\left(\overline{\mathcal{Z}}_{\mathcal{T}} ; \operatorname{Hom}\left(\gamma_{\mathcal{F}}^{*} \otimes L_{h}^{\otimes 2} \oplus \gamma_{\mathcal{F}}^{*} \otimes L_{h}^{\otimes 3} ; \gamma_{\mathcal{F}}^{*} \otimes \mathrm{ev}_{\widehat{0}}^{*} T \mathbb{P}^{3}\right)\right), \\
\overline{\mathcal{Z}}_{\mathcal{T}} \subset \mathbb{P} \mathcal{F} \times \mathcal{S}_{\overline{\mathcal{T}}}(\mu), \quad \mathcal{F}=L_{\tilde{1}} \oplus L_{\widetilde{1}}^{*} \longrightarrow \overline{\mathfrak{M}}_{\{\tilde{1}, h\} \sqcup M_{\tilde{1} \mathcal{T}},} \\
\alpha_{1}\left(v_{2}, v_{3}\right)=\mathcal{D}_{\overline{\mathcal{T}}, h}^{(2)} v_{2}+\mathcal{D}_{\overline{\mathcal{T}}, h}^{(3)} v_{3} .
\end{gathered}
$$

If $M_{\widetilde{1}} \mathcal{T}=\{\widehat{1}, l\}$ for some $l \in[N], \alpha_{1}$ has full rank over $\overline{\mathcal{Z}}_{\mathcal{T}}$ and thus

$$
\sum_{M_{\tilde{1}} \mathcal{T}=\{\hat{1}, l\}} \mathcal{C}_{\mathcal{Z}_{\mathcal{T}}}\left(\mathcal{D}_{\tilde{1}, \hat{1}}\right)=3 \sum_{l \in[N]}\left\langle 3 \lambda_{\mathcal{F}}-2 \lambda_{\mathcal{F}}, \overline{\mathcal{Z}}_{\mathcal{T}}\right\rangle\left|\mathcal{S}_{\overline{\mathcal{T}}}(\mu)\right|=3\left|\mathcal{S}_{1 ; 1}(\mu)\right| .
$$

If $M_{\tilde{1}} \mathcal{T}=\{\widehat{1}\}, \overline{\mathcal{Z}}_{\mathcal{T}} \approx \overline{\mathcal{S}}_{1}(\mu)$, and

$$
\mathcal{C}_{\mathcal{Z}_{\mathcal{T}}}\left(\mathcal{D}_{\widetilde{1}, \hat{1}}\right)=3 N\left(\alpha_{1}\right)=\left\langle 12 a_{\widehat{0}}+15 c_{1}\left(\mathcal{L}_{\widetilde{1}}^{*}\right), \overline{\mathcal{S}}_{1}(\mu)\right\rangle-9\left|\mathcal{S}_{2}(\mu)\right| ;
$$

see [17. Lemma 5.12]. The claim follows from equations (5.2)-(5.5).

Lemma 5.2 Suppose $\mathcal{T}=\left(M_{1}, I ; j, \underline{d}\right)$ is a bubble type such that $\mathcal{T}<\mathcal{T}_{1}$, $\chi_{\mathcal{T}}(\widetilde{1}, \widehat{1})=0$, and $\left|\chi_{\widetilde{1}}(\mathcal{T})\right|=2$.

(1) If $\left|\widehat{I}^{+}\right|>\left|\chi_{\widetilde{1}}(\mathcal{T})\right|, \mathbb{P}\left(L_{\widetilde{1}} \oplus L_{\widetilde{1}}^{*}\right) \mid \mathcal{S}_{\mathcal{T} \mid \mathcal{T}_{1}}(\mu)$ is a finite union of $\mathcal{D}_{\widetilde{1}, \hat{1}}$-hollow subsets and thus

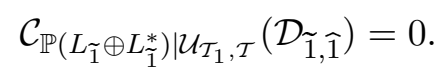

(2) If $\left|\widehat{I}^{+}\right|=\left|\chi_{\widetilde{1}}(\mathcal{T})\right|, M_{\tilde{1}} \mathcal{T}=\{\widehat{1}\}$ if $\mathcal{S}_{\mathcal{T} \mid \mathcal{T}_{1}}(\mu) \neq \emptyset$, and

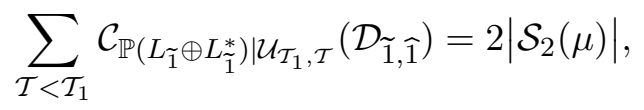


where the sum is taken over all equivalence classes of bubble types of the above form.

Proof The proof is a mixture of the proof of Lemma 5.1 with (2) of the proof of Lemma 4.12 thus, we omit it.

Corollary 5.3 The total contribution from the boundary strata $\mathcal{U}_{\mathcal{T}_{1}, \mathcal{T}}$ such that $\chi_{\mathcal{T}}(\widetilde{1}, \widehat{1})=0$ to the number $\mathcal{C}_{\partial \mathbb{P}\left(L_{\widetilde{1}} \oplus L_{\widetilde{1}}^{*}\right)}\left(\mathcal{D}_{\widetilde{1}, \widehat{1}}\right)$ is given by

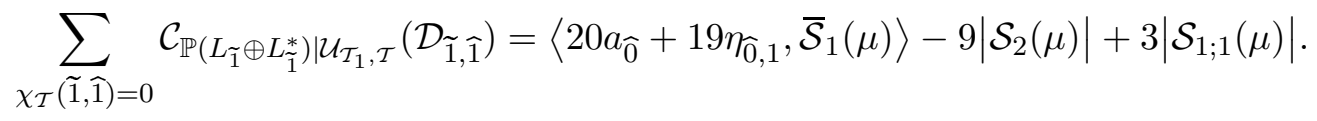

Proof This Corollary follows immediately from Lemmas 5.1 and 5.2 .

\subsection{Contributions from the spaces $\mathcal{U}_{\mathcal{T} \mid \mathcal{T}_{1}}$ with $\chi_{\mathcal{T}}(\widetilde{1}, \widehat{1})>0$}

In this subsection, we prove Corollary 5.9. which gives the total contribution to the number $\mathcal{C}_{\partial \mathbb{P}\left(L_{\widetilde{1}} \oplus L_{\widetilde{1}}^{*}\right)}\left(\mathcal{D}_{\widetilde{1}, \widehat{1}}\right)$ from the spaces $\mathcal{U}_{\mathcal{T}_{1}, \mathcal{T}}$, where

$$
\mathcal{T} \equiv\left(M_{1}, I ; j, \underline{d}\right)<\mathcal{T}_{1}
$$

is a bubble type such that $\chi_{\mathcal{T}}(\widetilde{1}, \widehat{1})>0$. Note that by the last paragraph of Subsection [5.1 it is sufficient to consider bubble types $\mathcal{T}$ such that $j_{\widehat{1}}>\widetilde{1}$.

Figure 13 shows the three types of boundary strata $\overline{\mathcal{V}}_{1}^{(1)}(\mu) \cap \mathcal{U}_{\mathcal{T}_{1}, \mathcal{T}}$ such that

$$
\mathbb{P}\left(L_{\tilde{1}} \oplus L_{\widetilde{1}}^{*}\right) \mid \overline{\mathcal{V}}_{1}^{(1)}(\mu) \cap \mathcal{U}_{\mathcal{T}_{1}, \mathcal{T}}
$$

is not contained in a finite union of $\mathcal{D}_{\tilde{1}, \hat{1}}$-hollow sets. As in Subsection 5.2 , we have to split each space

$$
\mathbb{P}\left(L_{\tilde{1}} \oplus L_{\widetilde{1}}^{*}\right) \mid \overline{\mathcal{V}}_{1}^{(1)}(\mu) \cap \mathcal{U}_{\mathcal{T}_{1}, \mathcal{T}}
$$

into two or three subspaces, as indicated on the right-hand side of Figure 13 . Lemma 5.4 computes the contributions from the first two types of boundary strata of Figure [13] Lemma [5.7 deals with remaining one.

Lemma 5.4 Suppose $\mathcal{T}=\left(M_{1}, I ; j, \underline{d}\right)$ is a bubble type such that $\mathcal{T}<\mathcal{T}_{1}$, $\chi_{\mathcal{T}}(\widetilde{1}, \widehat{1})>0$, and $d_{\widetilde{1}}=0$. (1) If $\left|\widehat{I}^{+}\right|>\left|\chi_{\widetilde{1}}(\mathcal{T})\right|, \mathbb{P}\left(L_{\widetilde{1}} \oplus L_{\widetilde{1}}^{*}\right) \mid \mathcal{U}_{\mathcal{T} \mid \mathcal{T}_{1}}^{(1)}(\mu)$ is a finite union of $\mathcal{D}_{\tilde{1}, \hat{1}}-$ hollow subspaces and thus

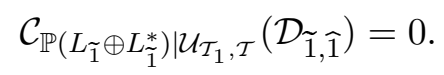




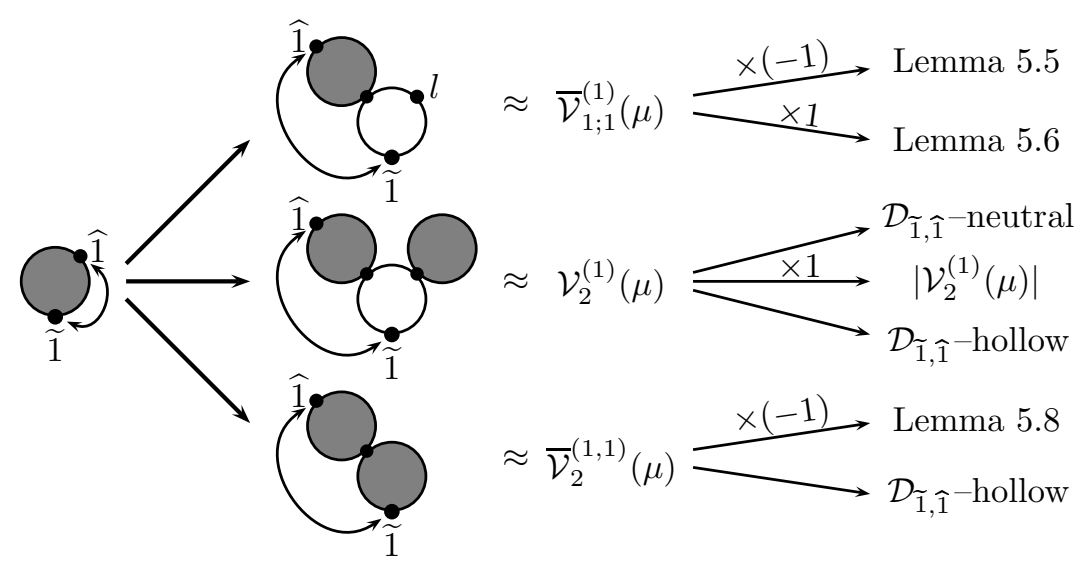

Figure 13: An outline of Subsection 5.3

(2) The total contribution from the boundary strata $\mathcal{U}_{\mathcal{I}_{1}, \mathcal{I}}$ such that $\left|\widehat{I}^{+}\right|=$ $\left|\chi_{\widetilde{1}}(\mathcal{T})\right|=1$ is given by

$$
\sum_{\left|\widehat{I}^{+}\right|=\left|\chi_{\tilde{1}}(\mathcal{T})\right|=1} \mathcal{C}_{\mathbb{P}\left(L_{\widetilde{1}} \oplus L_{\widetilde{1}}^{*}\right) \mid \mathcal{U}_{\mathcal{T}_{1}, \mathcal{T}}}\left(\mathcal{D}_{\widetilde{1}, \hat{1}}\right)=\left\langle c_{1}\left(L_{\widetilde{1}}^{*}\right), \overline{\mathcal{V}}_{1 ; 1}^{(1)}(\mu)\right\rangle-3\left|\mathcal{S}_{1 ; 1}(\mu)\right| .
$$

(3) The total contribution from the boundary strata $\mathcal{U}_{\mathcal{T}_{1}, \mathcal{T}}$ such that $\left|\widehat{I}^{+}\right|=$ $\left|\chi_{\tilde{1}}(\mathcal{T})\right|=2$ is given by

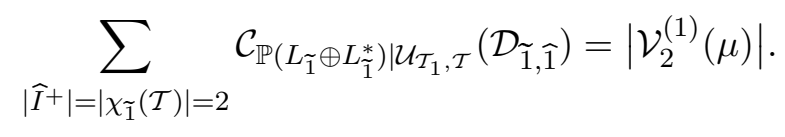

Proof (1) By Lemma 4.1

$$
\overline{\mathcal{U}}_{\mathcal{T}_{1}}^{(1)}(\mu) \cap \mathcal{U}_{\mathcal{T}_{1}, \mathcal{T}} \subset \mathcal{U}_{\mathcal{T} \mid \mathcal{T}_{1}}^{(1)}(\mu)
$$

With appropriate identifications, $\mathcal{U}_{\mathcal{T} \mid \mathcal{T}_{1}}^{(1)}(\mu)$ is the zero set of the section

$$
\mathrm{ev}_{\mathcal{T}_{1}, M_{0}} \oplus\left(\mathrm{ev}_{\widehat{1}}-\mathrm{ev}_{\tilde{1}}\right)
$$

of the bundle

$$
\operatorname{ev}_{\mathcal{T}_{1}, M_{0}}^{*} \mathcal{N} \Delta_{\mathcal{T}_{1}}(\mu) \oplus \operatorname{ev}_{\widetilde{1}}^{*} T \mathbb{P}^{3}
$$

over an open neighborhood of $\mathcal{U}_{\mathcal{T} \mid \mathcal{T}_{1}}^{(1)}(\mu)$ in $\mathcal{U}_{\mathcal{T}_{1}, \mathcal{T}}$. By Lemma 3.4 this section is transversal to the zero set. By Proposition 3.5 there exists a $C^{1}$-negligible 
map

$$
\varepsilon_{-}: \mathcal{F} \mathcal{T}_{\delta}-Y\left(\mathcal{F} \mathcal{T} ; \widehat{I}^{+}\right) \longrightarrow \mathrm{ev}_{\mathcal{T}_{1}, M_{0}}^{*} \mathcal{N} \Delta_{\mathcal{T}_{1}}(\mu) \oplus \operatorname{ev}_{\widehat{0}}^{*} T \mathbb{P}^{3}
$$

such that

$$
\left\{\mathrm{ev}_{\mathcal{T}_{1}, M_{0}} \times \mathrm{ev}_{\widetilde{1}} \times \mathrm{ev}_{\widehat{1}}\right\}\left(\phi_{\mathcal{T}_{1}, \mathcal{T}}(b ; v)\right)=\left\{\mathrm{ev}_{\mathcal{T}_{1}, M_{0}} \times \mathrm{ev}_{\widetilde{1}} \times \mathrm{ev}_{\widehat{1}}\right\}(b)+\varepsilon_{-}(b ; v)
$$

for all $(b ; v) \in \mathcal{F} \mathcal{T}_{\delta}-Y\left(\mathcal{F} \mathcal{T} ; \widehat{I}^{+}\right)$. On the other hand, by Proposition 3.5

$$
\mathcal{D}_{\widetilde{1}, \widehat{1}} \phi_{\mathcal{T}_{1}, \mathcal{T}}\left(\left[v_{\tilde{1}}, v_{\widehat{1}}\right] ; v\right)=\{\alpha+\varepsilon(v)\} \rho(v) \quad \text { for all } v \in \mathcal{F} \mathcal{T}_{\delta}-Y\left(\mathcal{F} \mathcal{T} ; \widehat{I}^{+}\right) .
$$

In this equation, $\rho$ is the monomials map on $\mathcal{F} \mathcal{T}$ defined by

$$
\rho_{h}(v)= \begin{cases}\rho_{\mathcal{T}}^{(1), \widehat{1} ; \widehat{1}}(v), & \text { if } h \in \chi_{\widehat{j}_{\widehat{1}}}(\mathcal{T})-\chi_{\widehat{1}}(\mathcal{T}) ; \\ \rho_{\mathcal{T}}^{(1 ; 1), \widehat{1} ; h}(v), & \text { if } h \in \chi_{\widehat{1}}(\mathcal{T}) ; \\ \rho_{\mathcal{T}, \widetilde{1} ; h}^{(1 ; 1)}(v), & \text { if } h \in \chi_{\widetilde{1}}(\mathcal{T}), h \not \leq j_{\widehat{1}} ;\end{cases}
$$

with values in the bundle $\tilde{\mathcal{F}} \mathcal{T}=\bigoplus_{h \in \tilde{I}} \widetilde{\mathcal{F}}_{h} \mathcal{T}$, where

$$
\begin{aligned}
& \widetilde{I}=\chi_{j_{\widehat{1}}}(\mathcal{T}) \cup\left\{h \in \chi_{\widetilde{1}}(\mathcal{T}): h \not \leq j_{\widehat{1}}\right\}, \\
& \widetilde{\mathcal{F}}_{h} \mathcal{T}= \begin{cases}\widetilde{\mathcal{F}}_{\mathcal{T}, \widehat{i}, \hat{1}}^{(1)} \mathcal{T}, & \text { if } h \in \chi_{j_{\widehat{1}}}(\mathcal{T})-\chi_{\widehat{1}}(\mathcal{T}) ; \\
\widetilde{\mathcal{F}}_{\mathcal{T}}^{(1 ; 1)} \mathcal{T}, \quad \text { if } h \in \chi_{\widehat{1}}(\mathcal{T}) ; \\
\widetilde{\mathcal{F}}_{\mathcal{T}, \tilde{1} ;: h}^{(1 ; 1)} \mathcal{T}, \quad \text { if } h \in \chi_{\widetilde{1}}(\mathcal{T}), h \not \leq j_{\widehat{1}} .\end{cases}
\end{aligned}
$$

The linear map $\alpha: \tilde{\mathcal{F}} \mathcal{T} \longrightarrow \gamma_{L_{\tilde{1}} \oplus L_{\widetilde{1}}^{*}}^{*} \otimes \mathrm{ev}_{\widehat{0}}^{*} T \mathbb{P}^{3}$ is given by

$$
\alpha\left(\left[v_{\widetilde{1}}, v_{\widehat{1}}\right], \widetilde{v}_{h}\right)= \begin{cases}v_{\widehat{1}} \otimes \mathcal{D}_{\mathcal{T}, j_{\hat{1}}^{*}(\mathcal{T})}^{(1)} \widetilde{v}_{h}, & \text { if } h \in \chi_{j_{\widehat{1}}}(\mathcal{T})-\chi_{\widehat{1}}(\mathcal{T}) ; \\ -v_{\widehat{1}} \otimes\left(y_{h ; \hat{1}}-x_{\widehat{1}, h}\right)^{-2} \otimes \mathcal{D}_{\mathcal{T}, h}^{(1)} \widetilde{v}_{h}, & \text { if } h \in \chi_{\widehat{1}}(\mathcal{T}) ; \\ v_{\widetilde{1}} \otimes \mathcal{D}_{\mathcal{T}, h}^{(1)} \widetilde{v}_{h}, & \text { if } h \in \chi_{\widehat{1}}(\mathcal{T}), h \not j_{\widehat{1}} .\end{cases}
$$

In particular, by Lemma 3.4, $\alpha$ has full rank over $\mathcal{U}_{\mathcal{T} \mid \mathcal{T}_{1}}^{(1)}(\mu)$ outside of the set

$$
\mathcal{Z}_{\mathcal{T}} \equiv \begin{cases}\mathbb{P} L_{\widetilde{1}}, & \text { if } h \leq j_{\widehat{1}} \text { for all } h \in \chi_{\widetilde{1}}(\mathcal{T}) \\ \mathbb{P} L_{\tilde{1}} \cup \mathbb{P} L_{\widetilde{1}}^{*}, & \text { otherwise. }\end{cases}
$$

As usual,

$$
\varepsilon: \mathcal{F} \mathcal{T}-Y\left(\mathcal{F} \mathcal{T} ; \widehat{I}^{+}\right) \longrightarrow \operatorname{Hom}\left(\widetilde{\mathcal{F}} \mathcal{T}, \gamma_{L_{\tilde{1}} \oplus L_{\widehat{1}}^{*}}^{*} \otimes \mathrm{ev}_{\widehat{0}}^{*} T \mathbb{P}^{3}\right)
$$

is a $C^{0}$-negligible map. Thus,

$$
\mathbb{P}\left(L_{\widetilde{1}} \oplus L_{\widetilde{1}}^{*}\right) \mid \mathcal{U}_{\mathcal{T} \mid \mathcal{T}_{1}}^{(1)}(\mu)-\mathcal{Z}_{\mathcal{T}}
$$


is $\mathcal{D}_{\widetilde{1}, \hat{1}}$-hollow unless $\widehat{I}^{+}=\widetilde{I}$. Since $\chi_{\mathcal{T}}(\widetilde{1}, \widehat{1})>0$ and $d_{\widetilde{1}}=0$, if $\widehat{I}^{+}=\widetilde{I}$ and $\mathcal{U}_{\mathcal{T} \mid \mathcal{T}_{1}}(\mu) \neq \emptyset$, either $\mathcal{T}=\mathcal{T}_{1}(l)$ for some $l \in[N]$ or $\left|\widehat{I}^{+}\right|=\left|\chi_{\widetilde{1}}(\mathcal{T})\right|=2$ and $M_{\widetilde{1}} \mathcal{T}=\emptyset$. In the second case, the matrix corresponding to the monomials $\operatorname{map} \rho$ is

$$
\left(\begin{array}{cc}
-1 & 0 \\
0 & 1
\end{array}\right)
$$

Thus, $\rho$ is neutral, and

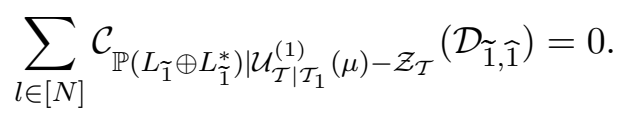

In the first case, the degree of $\rho$ is -1 . Thus, by Proposition 2.18B a rescaling of the linear map, and the decomposition (3.4), we obtain

$$
\begin{gathered}
\sum_{\left|\widehat{I}^{+}\right|=\left|\chi_{\tilde{1}}(\mathcal{T})\right|=1} \mathcal{C}_{\mathbb{P}\left(L_{\widetilde{1}} \oplus L_{\widetilde{1}}^{*}\right) \mid \mathcal{U}_{\mathcal{T}_{1}(l) \mid \mathcal{T}_{1}}^{(1)}(\mu)-\mathcal{Z}_{\mathcal{T}}}\left(\mathcal{D}_{\widetilde{1}, \hat{1}}\right)=-N\left(\alpha_{1}\right), \quad \text { where } \\
\alpha_{1} \in \Gamma\left(\mathbb{P}^{1} \times \overline{\mathcal{V}}_{1 ; 1}^{(1)}(\mu) ; \operatorname{Hom}\left(\gamma^{*} \otimes L_{\widetilde{1}}^{*}, \gamma^{*} \otimes \operatorname{ev}_{\widehat{0}}^{*} T \mathbb{P}^{3}\right)\right), \quad \alpha_{1} \mid \mathcal{U}_{\mathcal{T}_{1} / l}^{(1)}(\mu)=\mathcal{D}_{\mathcal{T}_{1} / l, \hat{1}}^{(1)} .
\end{gathered}
$$

Thus, by Lemma 5.5

$$
\begin{aligned}
\sum_{l \in[N]} \mathcal{C}_{\mathbb{P}\left(L_{\widetilde{1}} \oplus L_{\widetilde{1}}^{*}\right) \mid \mathcal{U}_{\mathcal{T}_{1}(l) \mid \mathcal{T}_{1}}^{(1)}(\mu)-\mathcal{Z}_{\mathcal{T}}}\left(\mathcal{D}_{\widetilde{1}, \widehat{1}}\right)=-\left\langle 4 a_{\widehat{0}}-c_{1}\left(L_{\widetilde{1}}^{*}\right), \overline{\mathcal{V}}_{1 ; 1}^{(1)}(\mu)\right\rangle \\
+2\left|\mathcal{S}_{1 ; 1}(\mu)\right|-\left|\mathcal{V}_{2 ; 1}^{(1,1)}(\mu)\right| .
\end{aligned}
$$

The space $\mathcal{V}_{2 ; 1}^{(1,1)}(\mu)$ is the disjoint union of sets

$$
\mathcal{U}_{\mathcal{T}}^{(1)}(\mu) \equiv\left\{b \in \mathcal{U}_{\mathcal{T}}(\mu): \operatorname{ev}_{\widehat{1}}(b)=\operatorname{ev}_{\widehat{2}}(b)\right\}
$$

taken over all bubble types $\mathcal{T}=\left(M_{2}-\{l\}, I_{2}(l) ; j, \underline{d}\right)$, where

$$
I_{2}(l)=\{\widehat{0}=l\} \sqcup I_{2}^{+}, \quad j_{\widehat{1}}=\widetilde{1}, \quad j_{\widehat{2}}=\widetilde{2}, \quad d_{\widetilde{1}}, d_{\widetilde{2}}>0, \quad \text { and } \quad d_{\widetilde{1}}+d_{\widetilde{2}}=d .
$$

The image of every element of $\mathcal{V}_{2 ; 1}^{(1,1)}(\mu)$ has two components arranged in a circle, as in the fifth picture of Figure 1 with one of the two nodes lying on one of the constraints $\mu_{1}, \ldots, \mu_{N}$.

(3) We next consider the contribution from the space $\mathbb{P} L_{\widetilde{1}} \mid \mathcal{U}_{\mathcal{T} \mid \mathcal{T}_{1}}^{(1)}(\mu)$. We model a neighborhood of $\mathbb{P} L_{\widetilde{1}}$ in $\mathbb{P}\left(L_{\widetilde{1}} \oplus L_{\widetilde{1}}^{*}\right)$ by the map

$$
L_{\widetilde{1}}^{*} \otimes L_{\widetilde{1}}^{*} \longrightarrow \mathbb{P}\left(L_{\widetilde{1}} \oplus L_{\widetilde{1}}^{*}\right), \quad\left(\left[v_{\widetilde{1}}, v_{\widehat{1}}\right], u\right) \longrightarrow\left[v_{\widetilde{1}}, u\left(v_{\widetilde{1}}\right)\right] .
$$


In this case, with notation as in $(2)$ above, $\widetilde{I}=\chi_{j_{\widehat{1}}}(\mathcal{T}) \sqcup \chi_{\widetilde{1}}(\mathcal{T})$,

$$
\left(\rho_{h}(u, v), \alpha\left(\widetilde{v}_{h}\right)\right)= \begin{cases}\left(u \otimes \rho_{\mathcal{T}, \widehat{1}, \widehat{1}}^{(1)}(v), \mathcal{D}_{\mathcal{T}, j_{\widehat{1}}^{*}(\mathcal{T})}^{(1)} \widetilde{v}_{h}\right), & \text { if } h \in \chi_{j_{\widehat{1}}}(\mathcal{T})-\chi_{\widehat{1}}(\mathcal{T}) ; \\ \left(u \otimes \rho_{\mathcal{T}, \widehat{1} ; h}^{(; ;)}(v),\right. & \text { if } h \in \chi_{\widehat{1}}(\mathcal{T}) ; \\ \left.-\left(y_{h ; \hat{1}}-x_{\widehat{1} ; h}\right)^{-2} \otimes \mathcal{D}_{\mathcal{T}, h}^{(1)} \widetilde{v}_{h}\right), & \text { if } h \in \chi_{\widetilde{1}}(\mathcal{T}) .\end{cases}
$$

As before $\alpha$ has full rank on $\mathbb{P} L_{\widetilde{1}} \mid \mathcal{U}_{\mathcal{T} \mid \mathcal{T}_{1}}^{(1)}(\mu)$. Thus, $\mathbb{P} L_{\widetilde{1}} \mid \mathcal{U}_{\mathcal{T} \mid \mathcal{T}_{1}}^{(1)}(\mu)$ is $\mathcal{D}_{\widetilde{1}, \hat{1}}$-hollow unless either $\mathcal{T}=\mathcal{T}_{1}(l)$ for some $l \in[N]$ or $\left|\widehat{I}^{+}\right|=\left|\chi_{\widetilde{1}}(\mathcal{T})\right|=2$ and $M_{\tilde{1}} \mathcal{T}=\emptyset$. In both cases, the degree of $\rho$ is one. In the second case, $\alpha$ is an isomorphism on every fiber, and thus

$$
\sum_{\left|\hat{I}^{+}\right|=\left|\chi_{\tilde{1}}(\mathcal{T})\right|=2} \mathcal{C}_{\mathbb{P} L_{\tilde{1}} \mid \mathcal{U}_{\mathcal{T} \mid \mathcal{T}_{1}}^{(1)}(\mu)}\left(\mathcal{D}_{\tilde{1}, \hat{1}}\right)=\left|\mathcal{V}_{2}^{(1)}(\mu)\right| .
$$

In the first case, via the decomposition (3.4), we obtain

$$
\begin{gathered}
\sum_{l \in[N]} \mathcal{C}_{\mathbb{P} L_{\widetilde{1}} \mid \mathcal{U}_{\mathcal{T} \mid \mathcal{T}_{1}(l)}^{(1)}(\mu)}\left(\mathcal{D}_{\widetilde{1}, \hat{1}}\right)=N\left(\alpha_{1}\right), \quad \text { where } \\
\alpha_{1} \in \Gamma\left(\overline{\mathcal{V}}_{1 ; 1}^{(1)}(\mu) ; \operatorname{Hom}\left(L_{\widetilde{1}} \oplus L_{\widetilde{1}}^{*}, \operatorname{ev}_{\widehat{0}}^{*} T \mathbb{P}^{3}\right)\right), \\
\left\{\alpha_{1} \mid \mathcal{U}_{\mathcal{T}_{1 ; l}}^{(1)}(\mu)\right\}\left(v_{\widetilde{1}}, v_{\widehat{1}}\right)=\mathcal{D}_{\mathcal{T}_{1 ; l}, \widetilde{1}_{\tilde{1}}^{(1)}} v_{\widetilde{1}} \mathcal{D}_{\mathcal{T}_{1 ; l}, \hat{1}}^{(1)} v_{\widehat{1}} .
\end{gathered}
$$

Thus, by Lemma 5.6

$$
\sum_{l \in[N]} \mathcal{C}_{\mathbb{P} L_{\tilde{1}} \mid \mathcal{U}_{\mathcal{T} \mid \mathcal{T}_{1}(l)}}\left(\mathcal{D}_{\tilde{1}, \widehat{1}}\right)=\left\langle 4 a_{\widehat{0}}, \overline{\mathcal{V}}_{1 ; 1}^{(1)}(\mu)\right\rangle-5\left|\mathcal{S}_{1 ; 1}(\mu)\right|+\left|\mathcal{V}_{2 ; 1}^{(1,1)}(\mu)\right| .
$$

(4) Finally, it is easy to see that the set $\mathbb{P} L_{\widetilde{1}}^{*} \mid \mathcal{U}_{\mathcal{T} \mid \mathcal{T}_{1}}^{(1)}$ is $\mathcal{D}_{\widetilde{1}, \hat{1}}$-hollow. Indeed, in this case, the target bundle $\widetilde{\mathcal{F}} \mathcal{T}$ has the same rank as the target in case (2), but the domain of $\rho$ is $L_{\tilde{1}} \otimes L_{\tilde{1}} \oplus \mathcal{F} \mathcal{T}$, instead of $\mathcal{F} \mathcal{T}$. Thus, the claim follows from equations (5.6)-(5.9).

Lemma 5.5 If $\alpha_{1} \in \Gamma\left(\mathbb{P}^{1} \times \overline{\mathcal{V}}_{1 ; 1}^{(1)}(\mu) ; \operatorname{Hom}\left(\gamma^{*} \otimes L_{\widetilde{1}}^{*}, \gamma^{*} \otimes \mathrm{ev}_{\widehat{0}}^{*} T \mathbb{P}^{3}\right)\right)$ is given by $\alpha_{1} \mid \mathcal{U}_{\mathcal{T}_{1 ; l}^{(1)}}^{(\mu)}=\mathcal{D}_{\mathcal{T}_{1 ; l}, \hat{1}}^{(1)}$,

$$
N\left(\alpha_{1}\right)=\left\langle 4 a_{\widehat{0}}-c_{1}\left(L_{\widetilde{1}}^{*}\right), \overline{\mathcal{V}}_{1 ; 1}^{(1)}(\mu)\right\rangle-2\left|\mathcal{S}_{1 ; 1}(\mu)\right|+\left|\mathcal{V}_{2 ; 1}^{(1)}(\mu)\right| .
$$

Proof (1) By Propositions 2.18A and 2.18B

$$
N\left(\alpha_{1}\right)=\left\langle 4 a_{\widehat{0}}-c_{1}\left(L_{\widetilde{1}}^{*}\right), \overline{\mathcal{V}}_{1 ; 1}^{(1)}(\mu)\right\rangle-\mathcal{C}_{\mathbb{P}^{1} \times \partial \overline{\mathcal{V}}_{1 ; 1}^{(1)}(\mu)}\left(\alpha_{1}^{\perp}\right),
$$


where $\alpha_{1}^{\perp}$ denotes the composition of $\alpha_{1}$ with the projection map onto the quotient $\mathcal{O}_{1}$ of $\gamma^{*} \otimes \mathrm{ev}_{\widehat{0}}^{*} T \mathbb{P}^{3}$ by generic trivial line subbundle $\mathbb{C} \bar{\nu}_{1}$. Suppose $\mathcal{T}<\mathcal{T}_{1 ; l}$ is a bubble type such that

$$
\overline{\mathcal{V}}_{1 ; 1}^{(1)}(\mu) \cap \mathcal{U}_{\mathcal{T}_{1 ; l}, \mathcal{T}} \neq \emptyset .
$$

(2) If $\chi_{\mathcal{T}}(\widetilde{1}, \widehat{1})>0$, by Lemma 4.1

$$
\overline{\mathcal{V}}_{1 ; 1}^{(1)}(\mu) \cap \mathcal{U}_{\mathcal{T}_{1 ; l}, \mathcal{T}} \subset \mathcal{U}_{\mathcal{T} \mid \mathcal{T}_{1 ; l}}^{(1)}(\mu),
$$

and thus $\widehat{I}^{+}=\{h\}$ is a single-element set. Furthermore, $d_{\widetilde{1}} \neq 0$, since our constraints $\mu$ are disjoint. Thus, if $j_{\widehat{1}}=\widetilde{1}, \alpha_{1}$ extends over $\mathbb{P}^{1} \times \mathcal{U}_{\mathcal{T} \mid \mathcal{T}_{1 ; l}}^{(1)}(\mu)$, and this extension does not vanish by Lemma 3.4. It follows that $\mathbb{P}^{1} \times \mathcal{U}_{\mathcal{T}_{1 ; l}, \mathcal{T}}$ does not contribute to $\mathcal{C}_{\mathbb{P}^{1} \times \partial \overline{\mathcal{V}}_{1 ; 1}^{(1)}(\mu)}\left(\alpha_{1}^{\perp}\right)$. If $j_{\widehat{1}}=h$ and $d_{h}=0$, by Proposition 3.5. $\alpha_{1}$ again has a nonvanishing extension over $\mathbb{P}^{1} \times \mathcal{U}_{\mathcal{T} \mid \mathcal{T}_{1 ; l}}^{(1)}(\mu)$. Thus, we only need to consider the case $j_{\widehat{1}}=h$ and $d_{\tilde{1}}, d_{h}>0$. By Proposition [3.5.

$$
\alpha_{1}\left(\phi_{\mathcal{T}_{1 ; l}, \mathcal{T}}(v)\right)=\left\{\mathcal{D}_{\mathcal{T}, \widehat{1}}^{(1)}+\varepsilon(v)\right\} v^{*} \quad \text { for all } v \in \mathcal{F} \mathcal{T}_{\delta}-Y(\mathcal{F} \mathcal{T} ;\{h\}) .
$$

Since $\mathcal{D}_{\mathcal{T}, \widehat{1}}^{(1)}$ does not vanish on $\mathcal{U}_{\mathcal{T} \mid \mathcal{T}_{1 ; l}}^{(1)}(\mu)$, from Proposition 2.18B we conclude that

$$
\begin{gathered}
\sum_{\chi_{\mathcal{T}}(\widetilde{1}, \hat{1})>0} \mathcal{C}_{\mathbb{P}^{1} \times \mathcal{U}_{\mathcal{T}_{1 ; l}, \mathcal{T}}}\left(\alpha_{1}^{\perp}\right)=-N\left(\alpha_{2}\right) \cdot\left|\mathcal{V}_{2 ; 1}^{(1,1)}(\mu)\right|, \quad \text { where } \\
\alpha_{2} \in \Gamma\left(\mathbb{P}^{1} ; \operatorname{Hom}\left(\mathbb{C}, \mathbb{C}^{3} / \gamma\right)\right)
\end{gathered}
$$

is a nonvanishing section. Thus, by Proposition 2.18A

$$
\sum_{\chi_{\mathcal{T}}(\widetilde{1}, \hat{1})>0} \mathcal{C}_{\mathbb{P}^{1} \times \mathcal{U}_{\mathcal{T}_{1 ; l}, \mathcal{T}}}\left(\alpha_{1}^{\perp}\right)=-\left|\mathcal{V}_{2 ; 1}^{(1,1)}(\mu)\right| .
$$

(3) If $\chi_{\mathcal{T}}(\widetilde{1}, \widehat{1})=0$, by Lemma 4.2

$$
\overline{\mathcal{V}}_{1 ; 1}^{(1)}(\mu) \cap \mathcal{U}_{\mathcal{T}_{1 ; l}, \mathcal{T}} \subset \mathcal{S}_{\mathcal{T} \mid \mathcal{T}_{1 ; l}}(\mu) .
$$

Thus, $\widehat{I}^{+}=\{h\}$ is again a single-element set. Adding the expansion of $\operatorname{ev}_{\widetilde{1}} \times \mathrm{ev}_{\widehat{1}}$ of Lemma 4.2 times $\left(y_{\widehat{1}}-x_{h}\right)^{-1}$ to the expansion of $\mathcal{D}_{\mathcal{T}_{1 ; l}, \hat{1}}^{(1)}$ of $(3 \mathrm{c})$ of Proposition 3.5. we obtain

$$
\begin{gathered}
\alpha_{1}\left(\phi_{\mathcal{T}_{1 ; l}, \mathcal{T}}(v)\right)=-\left(y_{\widehat{1}}-x_{h}\right)^{-2} \otimes\left\{\mathcal{D}_{\mathcal{T}, h}^{(2)}+\varepsilon(v)\right\} v \otimes v \\
\text { for all } v \in \mathcal{F} \mathcal{T}_{\delta} \text { such that } \phi_{\mathcal{T}_{1 ; l}, \mathcal{T}}(v) \in \mathcal{V}_{1 ; 1}^{(1)}
\end{gathered}
$$


Thus, as in the second half of (2) of the proof of Lemma 4.8 we can conclude that

$$
\begin{gathered}
\sum_{\chi_{\mathcal{T}}(\widetilde{1}, \hat{1})=0} \mathcal{C}_{\mathbb{P}^{1} \times \mathcal{U}_{\mathcal{T}(l), \mathcal{T}}}\left(\alpha_{1}^{\perp}\right)=2 N\left(\alpha_{2}\right) \cdot\left|\mathcal{S}_{1 ; 1}(\mu)\right|, \quad \text { where } \\
\alpha_{2} \in \Gamma\left(\mathbb{P}^{1} ; \operatorname{Hom}\left(\mathbb{C}, \mathbb{C}^{3} / \gamma\right)\right)
\end{gathered}
$$

is a nonvanishing section. Thus, by Proposition 2.18A

$$
\sum_{\chi_{\mathcal{T}}(\widetilde{1}, \widehat{1})=0} \mathcal{C}_{\mathbb{P} 1} \times \mathcal{U}_{\mathcal{T}_{1 ; l}, \mathcal{T}}\left(\alpha_{1}^{\perp}\right)=2\left|\mathcal{S}_{1 ; 1}(\mu)\right| .
$$

The claim follows from equations (5.10)-(5.12).

Lemma 5.6 If $\alpha_{1} \in \Gamma\left(\overline{\mathcal{V}}_{1 ; 1}^{(1)}(\mu) ; \operatorname{Hom}\left(L_{\widetilde{1}} \oplus L_{\widetilde{1}}^{*}, \mathrm{ev}_{\widehat{0}}^{*} T \mathbb{P}^{3}\right)\right)$ is given by

$$
\left.\alpha_{1}\right|_{\mathcal{U}_{\mathcal{T}_{1 ; l}}^{(1)}(\mu)}\left(v_{\tilde{1}}, v_{\widehat{1}}\right)=\mathcal{D}_{\mathcal{T}_{1 ; l}, \tilde{1}^{(1)}} v_{\tilde{1}}+\mathcal{D}_{\mathcal{T}_{1 ; l}, \hat{1}}^{(1)} v_{\widehat{1}}
$$

for all $l \in[N]$,

$$
N\left(\alpha_{1}\right)=\left\langle 4 a_{\widehat{0}}, \overline{\mathcal{V}}_{1 ; 1}^{(1)}(\mu)\right\rangle-5\left|\mathcal{S}_{1 ; 1}(\mu)\right|+\left|\mathcal{V}_{2 ; 1}^{(1,1)}(\mu)\right| .
$$

Proof (1) Let $\mathcal{F}=L_{\tilde{1}} \oplus L_{\widetilde{1}}^{*}$. By Propositions 2.18A and 2.18B

$$
N\left(\alpha_{1}\right)=\left\langle 4 a_{\widehat{0}}, \overline{\mathcal{V}}_{1 ; 1}^{(1)}(\mu)\right\rangle-\mathcal{C}_{\mathbb{P} \mathcal{F} \mid \partial \overline{\mathcal{V}}_{1 ; 1}^{(1)}(\mu)}\left(\alpha_{1}^{\perp}\right),
$$

where $\widetilde{\alpha}_{1}^{\perp}$ denotes the composition of the section

$$
\widetilde{\alpha}_{1} \in \Gamma\left(\mathbb{P} \mathcal{F} ; \operatorname{Hom}\left(\gamma_{\mathcal{F}}, \pi_{\mathbb{P} \mathcal{F}}^{*} \operatorname{ev}_{\widehat{0}}^{*} T \mathbb{P}^{3}\right)\right),
$$

induced by $\alpha$, with the projection map onto the quotient $\mathcal{O}_{1}$ of $\pi_{\mathbb{P} \mathcal{F}}^{*} \operatorname{ev}_{\widehat{0}}^{*} T \mathbb{P}^{3}$ by generic trivial line subbundle $\mathbb{C} \bar{\nu}_{1}$. Suppose $\mathcal{T}<\mathcal{T}_{1 ; l}$ is a bubble type such that

$$
\overline{\mathcal{V}}_{1 ; 1}^{(1)}(\mu) \cap \mathcal{U}_{\mathcal{T}_{1 ; l}, \mathcal{T}} \neq \emptyset \text {. }
$$

(2) If $\chi_{\mathcal{T}}(\widetilde{1}, \widehat{1})>0$, then

$$
\overline{\mathcal{V}}_{1 ; 1}^{(1)}(\mu) \cap \mathcal{U}_{\mathcal{T}_{1 ; l}, \mathcal{T}} \subset \mathcal{U}_{\mathcal{T} \mid \mathcal{T}_{1 ; l}}^{(1)}(\mu),
$$

$\widehat{I}^{+}=\{h\}$ is a single-element set, and $d_{\tilde{1}} \neq 0$. As before, we only need to consider the case $j_{\widehat{1}}=h$ and $d_{\tilde{1}}, d_{h}>0$. By Proposition 3.5 . $\widetilde{\alpha}_{1}\left(\phi_{\mathcal{T}_{1 ; l}, \mathcal{T}}\left(\left[v_{\widetilde{1}}, v_{\widehat{1}}\right], v\right)\right)=\left\{v_{\widehat{1}} \otimes \mathcal{D}_{\mathcal{T}, \widehat{1}}^{(1)}+\varepsilon(v)\right\} v^{*} \quad$ for all $v \in \mathcal{F} \mathcal{T}_{\delta}-Y(\mathcal{F} \mathcal{T} ;\{h\})$, 
where $\varepsilon$ is a $C^{0}$-negligible map. Since the linear map $v_{\widehat{1}} \otimes \mathcal{D}_{\mathcal{T}, \hat{1}}^{(1)}$ does not vanish outside of the set $\mathcal{Z}_{\mathcal{T}}=\mathbb{P} L_{\tilde{1}}$, by Proposition 3.5 and a rescaling of the linear map,

$$
\begin{gathered}
\sum_{\chi_{\mathcal{T}}(\widetilde{1}, \widehat{1})>0} \mathcal{C}_{\mathbb{P} \mathcal{F} \mid \mathcal{U}_{\mathcal{T} \mid \mathcal{T}_{1 ; l}(1)}^{(1)}(\mu)-\mathcal{Z}_{\mathcal{T}}}\left(\alpha_{1}^{\perp}\right)=-N\left(\alpha_{2}\right) \cdot\left|\mathcal{V}_{2 ; 1}^{(1,1)}(\mu)\right|, \quad \text { where } \\
\alpha_{2} \in \Gamma\left(\mathbb{P}^{1} ; \operatorname{Hom}\left(\gamma^{*}, \gamma^{*} \otimes \mathbb{C}^{2}\right)\right)
\end{gathered}
$$

is a nonvanishing section. Thus, by Proposition 2.18A

$$
\sum_{\chi \mathcal{T}(\widetilde{1}, \widehat{1})>0} \mathcal{C}_{\mathbb{P} \mathcal{F} \mid \mathcal{U}_{\mathcal{T} \mid \mathcal{T}_{1 ; l}}^{(1)}(\mu)-\mathcal{Z}_{\mathcal{T}}}\left(\widetilde{\alpha}_{1}^{\perp}\right)=-\left|\mathcal{V}_{2 ; 1}^{(1,1)}(\mu)\right|
$$

On the other hand, we can model a neighborhood of $\mathcal{Z}_{\mathcal{T}}$ in $\mathbb{P} \mathcal{F}$ by the map

$$
L_{\widetilde{1}}^{*} \otimes L_{\widetilde{1}}^{*} \longrightarrow \mathbb{P} \mathcal{F}, \quad\left(\left[v_{\tilde{1}}, v_{\widehat{1}}\right], u\right) \longrightarrow\left[v_{\tilde{1}}, u\left(v_{\tilde{1}}\right)\right] .
$$

Since by Proposition 3.5

$$
\begin{gathered}
\widetilde{\alpha}_{1}\left(\phi_{\mathcal{T}_{1 ; l}, \mathcal{T}}\left(\left[v_{\tilde{1}}, v_{\widehat{1}}\right], u, v\right)\right)=\left\{\mathcal{D}_{\mathcal{T}, \widehat{1}}^{(1)}+\varepsilon_{1}(v)\right\} u \otimes v^{*}+\mathcal{D}_{\mathcal{T}, \tilde{1}}+\varepsilon_{1}(v) \\
\text { for all } v \in \mathcal{F} \mathcal{T}_{\delta}-Y(\mathcal{F} \mathcal{T} ;\{h\}),
\end{gathered}
$$

it follows that $\mathcal{Z}_{\mathcal{T}} \mid \mathcal{U}_{\mathcal{T} \mid \mathcal{T}_{1 ; l}}^{(1)}(\mu)$ is $\widetilde{\alpha}_{1}^{\perp}$-hollow. Thus,

$$
\sum_{\chi_{\mathcal{T}}(\widetilde{1}, \hat{1})>0} \mathcal{C}_{\mathbb{P} \mathcal{F} \mid \mathcal{U}_{\mathcal{T}_{1 ; l}, \mathcal{T}}}\left(\widetilde{\alpha}_{1}^{\perp}\right)=-\left|\mathcal{V}_{2 ; 1}^{(1,1)}(\mu)\right| .
$$

(3) If $\chi_{\mathcal{T}}(\widetilde{1}, \widehat{1})=0$,

$$
\overline{\mathcal{V}}_{1 ; 1}^{(1)}(\mu) \cap \mathcal{U}_{\mathcal{T}_{1 ; l}, \mathcal{T}} \subset \mathcal{S}_{\mathcal{T} \mid \mathcal{T}_{1 ; l}}(\mu)
$$

and $\widehat{I}^{+}=\{h\}$ is a single-element set. Subtracting the expansion of $\mathrm{ev}_{\widetilde{1}} \times \mathrm{ev}_{\widehat{1}}$ of Lemma 4.2 times

$$
\left(y_{\widehat{1}}-x_{h}\right) \quad \text { and times } \quad\left(y_{\widehat{1}}-x_{h}\right)^{-1}
$$

from the expansions of $\mathcal{D}_{\mathcal{T}_{1 ; l}, \tilde{1}}^{(1)}$ and $\mathcal{D}_{\mathcal{T}_{1 ; l}, \hat{1}}^{(1)}$ in Proposition 3.5. we obtain

$$
\begin{array}{r}
\widetilde{\alpha}_{1}\left(\phi_{\mathcal{T}_{1 ; l}, \mathcal{T}}\left(\left[v_{\tilde{1}}, v_{\widehat{1}}\right], v\right)\right)=-\left(y_{\widehat{1}}-x_{h}\right)^{-1} \otimes\left(v_{\tilde{1}} \otimes \mathcal{D}_{\mathcal{T}, h}^{(2)}+\varepsilon_{\tilde{1}}(v)\right) v^{\otimes 2} \\
-\left(y_{\widehat{1}}-x_{h}\right)^{-3} \otimes\left(v_{\widehat{1}} \otimes \mathcal{D}_{\mathcal{\mathcal { T }}, h}^{(2)}+\varepsilon_{\widehat{1}}(v)\right) v^{\otimes 2}
\end{array}
$$

for all $v \in \mathcal{F} \mathcal{T}_{\delta}$ such that $\phi_{\mathcal{T}_{1 ; l}, \mathcal{T}}(v) \in \mathcal{V}_{1 ; 1}^{(1)}$. Let

$$
\mathcal{Z}_{\mathcal{T}}=\left\{\left[v_{\tilde{1}}, v_{\widehat{1}}\right] \in \mathbb{P} \mathcal{F}: v_{\tilde{1}}+\left(y_{\widehat{1}}-x_{h}\right)^{-2} v_{\widehat{0}}=0 \in L_{\tilde{1}}\right\} .
$$


Similarly to the argument in (2) of the proof of Lemma 5.4 from the above we can conclude that

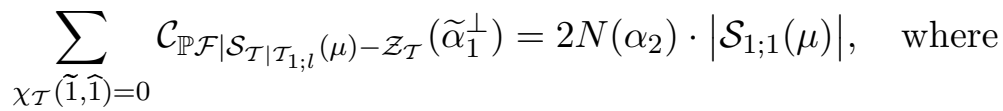

$$
\begin{aligned}
& \alpha_{2} \in \Gamma\left(\mathbb{P}^{1} ; \operatorname{Hom}\left(\gamma^{*}, \gamma^{*} \otimes \mathbb{C}^{2}\right)\right)
\end{aligned}
$$

is a nonvanishing section. Thus, by Lemma $2.18 \mathrm{~A}$

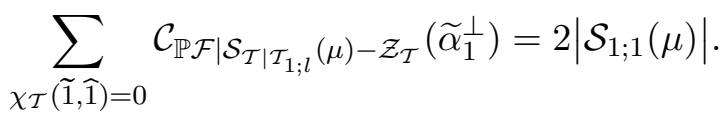

On the other hand, the same argument as in (3) of the proof of Lemma 5.4 shows that

$$
\mathcal{C}_{\mathcal{Z}_{\mathcal{T}} \mid \mathcal{S}_{\mathcal{T} \mid \mathcal{T}_{1 ; l}}(\mu)}\left(\widetilde{\alpha}_{1}\right)=3\left|\mathcal{Z}_{\mathcal{T}}\right|
$$

Thus, we conclude that

$$
\sum_{\chi_{\mathcal{T}}(\widetilde{1}, \hat{1})=0} \mathcal{C}_{\mathbb{P} \mathcal{F} \mid \mathcal{U}_{\mathcal{T}_{1 ; l}, \mathcal{T}}}\left(\widetilde{\alpha}_{1}^{\perp}\right)=5\left|\mathcal{S}_{1 ; 1}(\mu)\right| .
$$

The claim follows from equations (5.13)

Lemma 5.7 Suppose $\mathcal{T}=\left(M_{1}, I ; j, \underline{d}\right)$ is a bubble type such that $\mathcal{T}<\mathcal{T}_{1}$, $\chi_{\mathcal{T}}(\widetilde{1}, \widehat{1})>0$, and $d_{\tilde{1}}>0$.

(1) If $j_{\widehat{1}}=\widetilde{1},\left|\widehat{I}^{+}\right| \neq 1$, or $d_{j_{\widehat{1}}}=0, \mathbb{P}\left(L_{\widetilde{1}} \oplus L_{\widetilde{1}}^{*}\right) \mid \mathcal{U}_{\mathcal{T} \mid \mathcal{T}_{1}}^{(0)}$ is a finite union of $\mathcal{D}_{\widehat{1}, \hat{1}}-$ hollow subspaces and thus

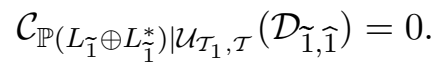

(2) The total contribution from the boundary spaces $\mathcal{U}_{\mathcal{T}_{1}, \mathcal{I}}$ such that $j_{\widehat{1}}>\widetilde{1}$, $\left|\widehat{I}^{+}\right|=1$, and $d_{j_{\widehat{1}}}>0$, is given by

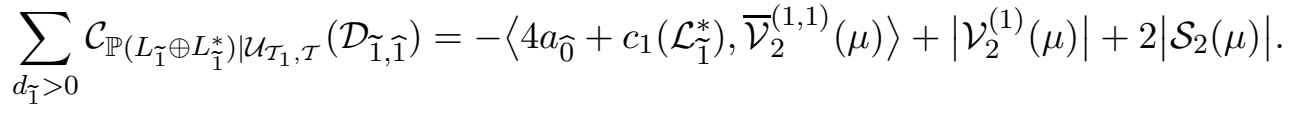

Proof (1) We proceed as in (1) of the proof of Lemma 5.4. In particular, we have

$$
\mathcal{D}_{\widetilde{1}, \widehat{1}} \phi_{\mathcal{T}_{1}, \mathcal{T}}\left(\left[v_{\tilde{1}}, v_{\widehat{1}}\right] ; v\right)=\{\alpha+\varepsilon(v)\} \rho(v) \quad \text { for all } v \in \mathcal{F} \mathcal{T}_{\delta}-Y\left(\mathcal{F} \mathcal{T} ; \widehat{I}^{+}\right) .
$$

In this equation, $\rho$ is the monomials map on $\mathcal{F} \mathcal{T}$ defined by

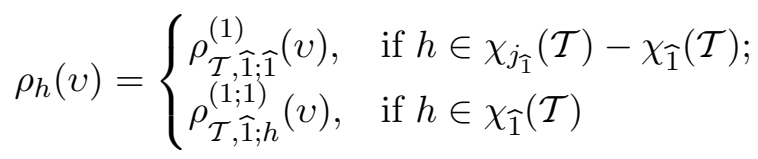


with values in the bundle

$$
\tilde{\mathcal{F}} \mathcal{T}=\bigoplus_{h \in \chi_{j_{\widehat{1}}}(\mathcal{T})} \widetilde{\mathcal{F}}_{h} \mathcal{T}, \quad \text { where } \quad \widetilde{\mathcal{F}}_{h} \mathcal{T}= \begin{cases}\widetilde{\mathcal{F}}_{\mathcal{T}}^{(1)}, \hat{i} ; \mathcal{T} & \text { if } h \in \chi_{j_{\widehat{1}}}(\mathcal{T})-\chi_{\widehat{1}}(\mathcal{T}) \\ \widetilde{\mathcal{F}}_{\mathcal{T}, \hat{1} ; h}^{(1 ; 1)} \mathcal{T}, & \text { if } h \in \chi_{\widehat{1}}(\mathcal{T})\end{cases}
$$

The linear map $\alpha: \tilde{\mathcal{F}} \mathcal{T} \longrightarrow \gamma_{L_{\tilde{1}}}^{*} L_{\widetilde{1}}^{*} \otimes \mathrm{ev}_{\widehat{0}}^{*} T \mathbb{P}^{3}$ is given by

$$
\alpha\left(\left[v_{\widetilde{1}}, v_{\widehat{1}}\right], \widetilde{v}_{h}\right)=v_{\widehat{1}} \otimes \begin{cases}\mathcal{D}_{\mathcal{T}, j_{\hat{1}}^{*}(\mathcal{T})}^{(1)} \widetilde{v}_{h}, & \text { if } h \in \chi_{j_{\widehat{1}}}(\mathcal{T})-\chi_{\widehat{1}}(\mathcal{T}) ; \\ -\left(y_{h ; \hat{1}}-x_{\widehat{1} ; h}\right)^{-2} \otimes \mathcal{D}_{\mathcal{T}, h}^{(1)} \widetilde{v}_{h}, & \text { if } h \in \chi_{\widehat{1}}(\mathcal{T}) .\end{cases}
$$

In particular, by Lemma 3.4 $\alpha$ has full rank over $\mathcal{U}_{\mathcal{T} \mid \mathcal{T}_{1}}^{(1)}(\mu)$ outside of the set $\mathcal{Z}_{\mathcal{T}} \equiv \mathbb{P} L_{\widetilde{1}}$. Thus,

$$
\mathbb{P}\left(L_{\widetilde{1}} \oplus L_{\widetilde{1}}^{*}\right) \mid \mathcal{U}_{\mathcal{T} \mid \mathcal{T}_{1}}^{(1)}(\mu)-\mathcal{Z}_{\mathcal{T}}
$$

is $\mathcal{D}_{\widetilde{1}, \hat{1}}$-hollow unless $\widehat{I}^{+}=\{h\}$ is a one-element set, $j_{\widehat{1}}=h$, and $d_{h} \neq 0$. If $\widehat{I}^{+}=\{h\}, j_{\widehat{I}}=h$, and $d_{h} \neq 0$, the degree of the map $\rho$ is -1 , and from Proposition 2.18B and a rescaling of the linear map,

$$
\begin{gathered}
\mathcal{C}_{\mathbb{P}\left(L_{\tilde{1}} \oplus L_{\widetilde{1}}^{*}\right) \mid \mathcal{U}_{\mathcal{T} \mid \mathcal{T}_{1}}^{(1)}(\mu)-\mathcal{Z}_{\mathcal{T}}}\left(\mathcal{D}_{\widetilde{1}, \widehat{1}}\right)=-N\left(\alpha_{1}^{\prime}\right), \quad \text { where } \\
\alpha_{1}^{\prime} \in \Gamma\left(\mathbb{P} \mathcal{F} ; \operatorname{Hom}\left(\gamma_{\mathcal{F}}^{*} \otimes L_{h}^{*}, \gamma_{\mathcal{F}}^{*} \otimes \operatorname{ev}_{\widehat{0}}^{*} T \mathbb{P}^{3}\right)\right), \\
\mathcal{F}=L_{\widetilde{1}} \oplus L_{\widetilde{1}}^{*} \longrightarrow \mathcal{U}_{\mathcal{T} \mid \mathcal{T}_{1}}^{(1)}(\mu), \quad \alpha_{1}^{\prime}=\mathcal{D}_{\mathcal{T}, \widehat{1}}^{(1)} .
\end{gathered}
$$

Thus, using Lemma [5.8, another rescaling of the linear map, and an obvious symmetry, we obtain

$$
\begin{aligned}
\sum_{\mathcal{T}} \mathcal{C}_{\mathbb{P}\left(L_{\widetilde{1}} \oplus L_{\widetilde{1}}^{*}\right) \mid \mathcal{U}_{\mathcal{T}_{1} \mid \mathcal{T}_{1}}^{(1)}(\mu)-\mathcal{Z}_{\mathcal{T}}}\left(\mathcal{D}_{\widetilde{1}, \hat{1}}\right)=-\left\langle 4 a_{\widehat{0}}\right. & \left.+c_{1}\left(\mathcal{L}_{\widetilde{1}}^{*}\right), \overline{\mathcal{V}}_{2}^{(1,1)}(\mu)\right\rangle \\
& +\left|\mathcal{V}_{2}^{(1)}(\mu)\right|+2\left|\mathcal{S}_{2}(\mu)\right| .
\end{aligned}
$$

Finally, it is easy to see that the set $\mathcal{Z}_{\mathcal{T}} \mid \mathcal{U}_{\mathcal{T} \mid \mathcal{T}_{1}}^{(1)}(\mu)$ is $\mathcal{D}_{\tilde{1}, \hat{1}}$-hollow for all bubble types $\mathcal{T}$.

Lemma 5.8 Suppose $\widetilde{\mathcal{T}}=\left(M_{2}, I_{2} ; \widetilde{j}, \widetilde{d}\right)$ is a bubble type such that

$$
\widetilde{j}_{\widehat{1}}=\widetilde{1}, \quad j_{\widehat{2}}=\widetilde{2}, \quad \widetilde{d}_{\tilde{1}}, \widetilde{d}_{\widetilde{2}}>0, \quad \text { and } \quad \widetilde{d}_{\widetilde{1}}+\widetilde{d}_{\widetilde{2}}=d,
$$

and $\mathcal{F} \longrightarrow \overline{\mathcal{U}}_{\tilde{\mathcal{T}}, \widetilde{\mathcal{T}}}$ is a rank-two vector bundle such that $c_{1}(\mathcal{F}) \mid \overline{\mathcal{U}}_{\widetilde{\mathcal{T}}}^{(1)}(\mu)=0$. If

$$
\alpha_{1} \in \Gamma\left(\mathbb{P} \mathcal{F} \mid \mathcal{U}_{\widetilde{\mathcal{T}}}^{(1)}(\mu) ; \operatorname{Hom}\left(\gamma^{*} \otimes L_{\tilde{1}} ; \gamma^{*} \otimes \mathrm{ev}_{\widetilde{0}}^{*} T \mathbb{P}^{3}\right)\right)
$$


is given by $\alpha_{1}\left(v_{\widetilde{1}}\right)=\mathcal{D}_{\widetilde{\mathcal{T}}, \widetilde{1}}^{(1)} v_{\widetilde{1}}$,

$$
N\left(\alpha_{1}\right)=\left\langle 4 a_{\widehat{0}}+c_{1}\left(\mathcal{L}_{\widetilde{1}}^{*}\right), \overline{\mathcal{U}}_{\widetilde{\mathcal{T}}}^{(1)}(\mu)\right\rangle-\left|\mathcal{U}_{\widetilde{\mathcal{T}} / \widehat{1}}^{(1)}(\mu)\right|-\left|\mathcal{U}_{\widetilde{\mathcal{T}} /\{\widehat{1}, \widehat{2}\}}(\mu)\right| .
$$

In other words, the sum of the numbers $N\left(\alpha_{1}\right)$ taken over all bubble types $\widetilde{\mathcal{T}}$ over the above form is given by

$$
\sum_{\tilde{\mathcal{T}}} N\left(\alpha_{1}\right)=\left\langle 4 a_{\widehat{0}}+c_{1}\left(\mathcal{L}_{\tilde{1}}^{*}\right), \overline{\mathcal{V}}_{2}^{(1,1)}(\mu)\right\rangle-\left|\mathcal{V}_{2}^{(1)}(\mu)\right|-2\left|\mathcal{S}_{2}(\mu)\right|
$$

Proof By Proposition $2.18 \mathrm{~A}$ and the assumption $c_{1}(\mathcal{F}) \mid \overline{\mathcal{U}}_{\widetilde{\mathcal{T}}}^{(1)}(\mu)=0$,

$$
N\left(\alpha_{1}\right)=\left\langle 4 a_{\widehat{0}}+c_{1}\left(L_{\widetilde{1}}^{*}\right), \overline{\mathcal{U}}_{\widetilde{\mathcal{T}}}^{(1)}(\mu)\right\rangle-\mathcal{C}_{\mathbb{P} \mathcal{F} \mid \partial \overline{\mathcal{U}}_{\tilde{\mathcal{T}}}^{(1)}(\mu)}\left(\alpha_{1}^{\perp}\right),
$$

where $\alpha_{1}^{\perp}$ denotes the composition of $\alpha_{1}$ with the projection map onto the quotient $\mathcal{O}_{1}$ of $\gamma^{*} \otimes \mathrm{ev}_{\widehat{0}}^{*} T \mathbb{P}^{3}$ by generic trivial line subbundle $\mathbb{C} \bar{\nu}_{1}$. Suppose $\mathcal{T}=\left(M_{2}, I ; j, \underline{d}\right)$ is a bubble type such that $\mathcal{T}<\widetilde{\mathcal{T}}$ and

$$
\overline{\mathcal{U}}_{\widetilde{\mathcal{T}}}^{(1)}(\mu) \cap \mathcal{U}_{\widetilde{\mathcal{T}}, \mathcal{T}} \neq \emptyset
$$

The section $\alpha_{1}$ extends over $\mathbb{P} \mathcal{F} \mid \partial \overline{\mathcal{U}}_{\widetilde{\mathcal{T}}}^{(1)}(\mu)$. Moreover, by Lemmas 4.3 and 3.4 this extension does not vanish unless $d_{\tilde{1}}=0$. Thus, in computing the number $\mathcal{C}_{\mathbb{P} \mathcal{F} \mid \partial \overline{\mathcal{U}}_{\tilde{\mathcal{T}}}^{(1)}(\mu)}\left(\alpha_{1}^{\perp}\right)$, we only need to consider bubble types $\mathcal{T}$ such that $d_{\widetilde{1}}=0$.

(2) If $d_{\widetilde{2}} \neq 0$, then

$$
\overline{\mathcal{U}}_{\widetilde{\mathcal{T}}}^{(1)}(\mu) \cap \mathcal{U}_{\widetilde{\mathcal{T}}, \mathcal{T}} \subset \mathcal{U}_{\mathcal{T} \mid \widetilde{\mathcal{T}}}^{(1)}(\mu)
$$

and $\mathcal{T}=\widetilde{\mathcal{T}}(\widehat{1})$ or $\mathcal{T}=\widetilde{\mathcal{T}}(l)$ for some $l \in[N] \cap M_{\widetilde{1}} \mathcal{T}$. Moreover, by Proposition 3.5

$$
\alpha_{1}\left(\phi_{\widetilde{\mathcal{T}}, \mathcal{T}}(v)\right)=\left\{\mathcal{D}_{\mathcal{T}, h}^{(1)}+\varepsilon(v)\right\} v \quad \text { for all } v \in \mathcal{F} \mathcal{T}_{\delta},
$$

if $h$ is the unique element of $\widehat{I}^{+}$. Thus, by the same argument as in (1) of the proof of Lemma 5.4, we conclude that

$$
\mathcal{C}_{\mathbb{P F} \mid \mathcal{U}_{\mathcal{T} \mid \tilde{\mathcal{T}}}^{(1)}(\mu)}\left(\alpha_{1}^{\perp}\right)=N\left(\alpha_{2}\right), \quad \text { where } \quad \alpha_{2} \in \Gamma\left(\mathbb{P}^{1} \times \overline{\mathcal{U}}_{\mathcal{T} \mid \widetilde{\mathcal{T}}}^{(1)}(\mu) ; \operatorname{Hom}(\mathbb{C}, \mathbb{C} / \gamma)\right)
$$

is a nonvanishing section. Thus, from Proposition 2.18A and the decomposition (3.4), we obtain

$$
\sum_{d_{\tilde{2}}>0} \mathcal{C}_{\mathbb{P} \mathcal{F} \mid \mathcal{U}_{\tilde{\mathcal{T}}, \mathcal{T}}}\left(\alpha_{1}^{\perp}\right)=\left|\mathcal{U}_{\tilde{\mathcal{T}} / \widehat{1}}^{(1)}(\mu)\right|+\sum_{l \in[N] \cap M_{\tilde{1}} \mathcal{T}}\left|\mathcal{U}_{\widetilde{\mathcal{T}} / l}^{(1)}(\mu)\right| .
$$


(3) If $d_{\widetilde{2}}=0$, then

$$
\overline{\mathcal{U}}_{\widetilde{\mathcal{T}}}^{(1)}(\mu) \cap \mathcal{U}_{\widetilde{\mathcal{T}}, \mathcal{T}} \subset \mathcal{S}_{\mathcal{T} \mid \widetilde{\mathcal{T}}}(\mu)
$$

$j_{\widehat{1}}=\widetilde{1}, j_{\widehat{2}}=\widetilde{2}, d_{\widetilde{1}}=d_{\widetilde{2}}=0$, and $\left|\widehat{I}^{+}\right|=2$. Furthermore,

$$
\alpha_{1}\left(\phi_{\widetilde{\mathcal{T}}, \mathcal{T}}(v)\right)=\left\{\mathcal{D}_{\mathcal{T}, h_{1}}^{(1)}+\varepsilon(v)\right\} v_{h_{1}} \quad \text { for all } v=\left(v_{h_{1}}, v_{h_{2}}\right) \in \mathcal{F} \mathcal{T}_{\delta},
$$

if $\widehat{I}^{+}=\left\{h_{1}, h_{2}\right\}$ and $h_{1}=j_{\widehat{1}}$. By an argument similar to (4) of Lemma 4.8, we conclude that

$$
\mathcal{C}_{\mathbb{P} \mathcal{F} \mid \mathcal{S}_{\mathcal{T} \mid \tilde{\mathcal{T}}}(\mu)}\left(\alpha_{1}^{\perp}\right)=N\left(\alpha_{2}\right), \quad \text { where } \quad \alpha_{2} \in \Gamma\left(\mathbb{P}^{1} \times \mathcal{S}_{\mathcal{T} \mid \widetilde{\mathcal{T}}}(\mu) ; \operatorname{Hom}(\mathbb{C}, \mathbb{C} / \gamma)\right)
$$

is a nonvanishing section. Thus, from Proposition 2.18A and the decomposition (3.4), we obtain

$$
\mathcal{C}_{\mathbb{P} \mathcal{F} \mid \mathcal{U}_{\widetilde{\mathcal{T}}, \mathcal{T}}}\left(\alpha_{1}^{\perp}\right)=\left|\mathcal{S}_{\widetilde{\mathcal{T}} /\{\hat{1}, \widehat{2}\}}(\mu)\right| .
$$

The claim follows by plugging equations (5.18) and (5.19) into (5.17) and using (3.2) and (3.3).

Remark By the second rescaling of the linear map referred to in the proof of Lemma 5.7 the number $\sum_{\widetilde{\mathcal{T}}} N\left(\alpha_{1}\right)$ of Lemma 5.7 does not change if we replace $\mathcal{D}_{\widetilde{\mathcal{T}}, \widetilde{1}}^{(1)}$ by $\mathcal{D}_{\widetilde{\mathcal{T}}, \widehat{1}}^{(1)}$. However, a direct computation, ie using Propositions 2.18A, $2.18 \mathrm{~B}$ and 3.5 gives a slightly different answer. As a result, we obtain yet another enumerative relationship:

$$
2\left\langle\eta_{\widehat{0}, 1}, \overline{\mathcal{V}}_{2}^{(1,1)}(\mu)\right\rangle=\left|\mathcal{V}_{2,(0,1)}^{(1,0,1)}(\mu)\right|
$$

Corollary 5.9 The total contribution from the boundary strata $\mathcal{U}_{\mathcal{T}_{1}, \mathcal{T}}$ such that $\chi_{\mathcal{T}}(\widetilde{1}, \widehat{1})>0$ to the number $\mathcal{C}_{\partial \mathbb{P}\left(L_{\widetilde{1}} \oplus L_{\widetilde{1}}^{*}\right)}\left(\mathcal{D}_{\widetilde{1}, \widehat{1}}\right)$ is given by

$$
\begin{aligned}
\sum_{\chi_{\mathcal{T}}(\widetilde{1}, \hat{1})=1} \mathcal{C}_{\mathbb{P}\left(L_{\tilde{1}} \oplus L_{\widehat{1}}^{*}\right) \mid \mathcal{U}_{\mathcal{1}_{1}, \mathcal{T}}}\left(\mathcal{D}_{\widetilde{1}, \hat{1}}\right)=\left\langle c_{1}\left(L_{\widetilde{1}}^{*}\right),\right. & \left.\overline{\mathcal{V}}_{1 ; 1}^{(1)}(\mu)\right\rangle-\left\langle 4 a_{\widehat{0}}+\frac{1}{2} \eta_{\widehat{0}, 1}, \overline{\mathcal{V}}_{2}^{(1,1)}(\mu)\right\rangle \\
& +2\left|\mathcal{V}_{2}^{(1)}(\mu)\right|+2\left|\mathcal{S}_{2}(\mu)\right|-3\left|\mathcal{S}_{1 ; 1}(\mu)\right|
\end{aligned}
$$

Proof This Corollary follows immediately from Lemmas 5.4 and 5.7 


\section{$6 \quad$ Level 1 numbers}

\subsection{Evaluation of cohomology classes on the spaces $\overline{\mathcal{V}}_{1}^{(1)}(\mu)$}

In this subsection, we evaluate various tautological classes on the space $\overline{\mathcal{V}}_{1}^{(1)}(\mu)$ and compute the other level 1 numbers of Lemma 1.8. We again use the computational method of Section 2 but first we represent each cohomology class by a vector-bundle section $s$ on neighborhood of $\overline{\mathcal{U}}_{\mathcal{T}_{1}}(\mu)$ in $\overline{\mathcal{U}}_{\mathcal{T}_{1}}$. We choose this section $s$ so that it is smooth and transversal to the zero set on all the strata of $\overline{\mathcal{U}}_{\mathcal{T}_{1}}(\mu)$, as well as on a finite number of natural submanifolds of the strata. We will impose additional restrictions on each given section to simplify our computations.

Lemma 6.1 With assumptions as in (2) of Lemma 1.8,

$$
\begin{array}{r}
\left\langle a_{\widehat{0}}, \overline{\mathcal{V}}_{1}^{(1)}(\mu)\right\rangle=\left\langle(2 d-6) a_{\widehat{0}}^{3}-4 a_{\widehat{0}}^{2} \eta_{\widehat{0}, 1}-a_{\widehat{0}} \eta_{\widehat{0}, 1}^{2}, \overline{\mathcal{V}}_{1}(\mu)\right\rangle+\left\langle a_{\widehat{0}}^{2}, \overline{\mathcal{V}}_{1}\left(\mu+H^{1}\right)\right\rangle \\
+\left\langle a_{\widehat{0}}, \overline{\mathcal{V}}_{2}(\mu)\right\rangle
\end{array}
$$

Proof (1) In this case, we choose a generic hyperplane $H^{2}$ in $\mathbb{P}^{3}$, instead of a section of $\operatorname{ev}_{\widehat{0}}^{*} \mathcal{O}\left(1_{\mathbb{P}^{3}}\right)$. Let $\widetilde{\mu}$ be the $\widetilde{M}=[N] \sqcup\{\widehat{0}\}$-tuple of constraints in $\mathbb{P}^{3}$ given by

$$
\widetilde{\mu}_{l}=\mu_{l} \quad \text { for all } l \in[N] ; \quad \widetilde{\mu}_{\widehat{0}}=H^{2} .
$$

By Proposition 3.5. $\mathcal{U}_{\mathcal{T}_{1}}(\widetilde{\mu})$ is a pseudovariety in $\overline{\mathcal{U}}_{\widetilde{\mathcal{T}}_{1}}$, and thus

$$
\mathrm{ev}_{\widetilde{1}} \times \mathrm{ev}_{\widehat{1}}: \mathcal{U}_{\widetilde{\mathcal{T}}_{1}}(\widetilde{\mu}) \longrightarrow \mathbb{P}^{3} \times \mathbb{P}^{3}
$$

is a 6-pseudocycle and determines the homology-intersection number

$$
\begin{aligned}
\left\langle\left\langle a_{\widehat{0}}, \overline{\mathcal{V}}_{1}^{(1)}(\mu)\right\rangle\right\rangle & \equiv\left\langle\left\langle\mathcal{V}_{1}^{(1)}(\widetilde{\mu})\right\rangle\right\rangle \equiv\left\langle\left\langle\left\{\mathrm{ev}_{\widetilde{1}} \times \mathrm{ev}_{\widehat{1}}\right\}^{-1}\left(\Delta_{\mathbb{P}^{3} \times \mathbb{P}^{3}}\right), \overline{\mathcal{U}}_{\mathcal{T}_{1}}(\widetilde{\mu})\right\rangle\right\rangle \\
& =\sum_{r+s=3}\left\langle\left\langle\left\{\mathrm{ev}_{\widetilde{1}} \times \mathrm{ev}_{\widehat{1}}\right\}^{-1}\left(H^{r} \times H^{s}\right), \overline{\mathcal{U}}_{\mathcal{T}_{1}}(\widetilde{\mu})\right\rangle\right\rangle, \\
& =2 d\left\langle a_{\widehat{0}}^{3}, \overline{\mathcal{V}}_{1}(\mu)\right\rangle+\left\langle a_{\widehat{0}}^{2}, \overline{\mathcal{V}}_{1}\left(\mu+H^{1}\right)\right\rangle .
\end{aligned}
$$

By the same argument as in Subsection 4.1

$$
\left\langle a_{\widehat{0}}, \overline{\mathcal{V}}_{1}^{(1)}(\mu)\right\rangle=\left|\mathcal{V}_{1}^{(1)}(\widetilde{\mu})\right|=\left\langle\left\langle a_{\widehat{0}}, \overline{\mathcal{V}}_{1}^{(1)}(\mu)\right\rangle\right\rangle-\mathcal{C}_{\partial \overline{\mathcal{U}}_{\mathcal{T}_{1}}(\widetilde{\mu})}\left(\mathrm{ev}_{\widetilde{1}} \times \mathrm{ev}_{\widehat{1}}, \Delta_{\mathbb{P}^{3} \times \mathbb{P}^{3}}\right),
$$

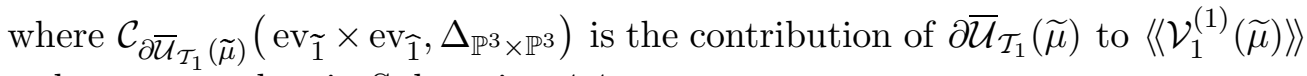
to be computed as in Subsection 4.4 
(2) If $\mathcal{T}=\left(M_{1}, I ; j, \underline{d}\right)<\mathcal{T}_{1}$ is a bubble type such that $\chi_{\mathcal{T}}(\widetilde{1}, \widehat{1})>0$, the map $\operatorname{ev}_{\tilde{1}} \times \operatorname{ev}_{\widehat{1}}$ is transversal to $\Delta_{\mathbb{P}^{3} \times \mathbb{P}^{3}}$ on $\mathcal{U}_{\mathcal{T} \mid \mathcal{T}_{1}}(\widetilde{\mu})$ by Lemma 3.4 . Thus, the image of $\mathcal{U}_{\mathcal{T} \mid \mathcal{T}_{1}}(\widetilde{\mu})$ is disjoint from $\Delta_{\mathbb{P}^{3} \times \mathbb{P}^{3}}$, and $\mathcal{U}_{\mathcal{T} \mid \mathcal{T}_{1}}$ does not contribute to

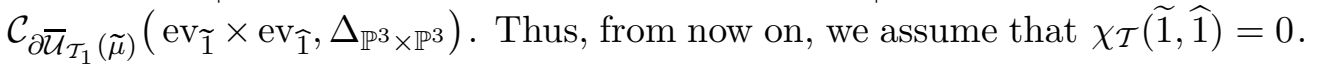
Note that $\mathcal{U}_{\mathcal{T} \mid \mathcal{T}_{1}}(\widetilde{\mu})=\emptyset$ unless $\left|\chi_{\tilde{1}}(\mathcal{T})\right| \in\{1,2\}$.

(3) With appropriate identifications, $\mathcal{U}_{\mathcal{T} \mid \mathcal{T}_{1}}(\widetilde{\mu})$ is the zero set of the section $\mathrm{ev}_{\mathcal{T}_{1}, \widetilde{M}}$ of the bundle $\mathrm{ev}_{\mathcal{T}_{1}, \widetilde{M}}^{*} \mathcal{N}_{\mathcal{T}_{1}}(\widetilde{\mu})$ over an open neighborhood of $\mathcal{U}_{\mathcal{T} \mid \mathcal{T}_{1}}(\widetilde{\mu})$ in $\mathcal{U}_{\mathcal{T}_{1}, \mathcal{T}}$. By Lemma 3.4, this section is transversal to the zero set. By Proposition [3.5] there exists a $C^{1}$-negligible map

$$
\varepsilon_{-}: \mathcal{F} \mathcal{T}_{\delta}-Y\left(\mathcal{F} \mathcal{T} ; \widehat{I}^{+}\right) \longrightarrow \mathrm{ev}_{\mathcal{T}_{1}, \widetilde{M}}^{*} \mathcal{N} \Delta_{\mathcal{T}_{1}}(\widetilde{\mu})
$$

such that

$$
\mathrm{ev}_{\mathcal{T}_{1}, \widetilde{M}}\left(\phi_{\mathcal{T}_{1}, \mathcal{T}}(b ; v)\right)=\mathrm{ev}_{\mathcal{T}, \widetilde{M}}(b)+\varepsilon_{-}(b ; v)
$$

for all $(b ; v) \in \mathcal{F} \mathcal{T}_{\delta}-Y\left(\mathcal{F} \mathcal{T} ; \widehat{I}^{+}\right)$. On the other hand, by Lemma 4.2

$$
\begin{gathered}
\left\{\mathrm{ev}_{\widetilde{1}} \times \mathrm{ev}_{\widehat{1}}\right\} \phi_{\mathcal{T}_{1}, \mathcal{T}}(v)=\sum_{h \in \chi_{\widetilde{1}}(\mathcal{T})}\left(y_{h ; \widehat{1}}-x_{\widehat{1} ; h}\right)^{-1} \otimes\left\{\mathcal{D}_{\mathcal{T}, h}^{(1)}+\varepsilon_{h}(v)\right\} \rho_{h}(v), \\
\text { where } \quad \rho_{h}(v)=\prod_{i \in\left(i_{\mathcal{T}}(h, \widehat{2}), h\right]} v_{i},
\end{gathered}
$$

for all $v \in \mathcal{F} \mathcal{T}_{\delta}$. Since the linear map,

$$
\mathcal{F} \equiv \bigoplus_{h \in \chi_{\tilde{1}}(\mathcal{T})} L_{h} \mathcal{T}, \quad v \longrightarrow \sum_{h \in \chi_{\tilde{1}}(\mathcal{T})} \mathcal{D}_{\mathcal{T}, h}^{(1)} v_{h},
$$

is injective over $\mathcal{U}_{\mathcal{T} \mid \mathcal{T}_{1}}(\widetilde{\mu})$ by Lemma 3.4. $\mathcal{U}_{\mathcal{T} \mid \mathcal{T}_{1}}$ is $\left(\operatorname{ev}_{\tilde{1}} \times \mathrm{ev}_{\hat{1}}, \Delta_{\mathbb{P}^{3} \times \mathbb{P}^{3}}\right)$-hollow unless $\chi_{\widetilde{1}}(\mathcal{T})=\widehat{I}^{+}$. If $\chi_{\widetilde{1}}(\mathcal{T})=\widehat{I}^{+}$, by Proposition 2.18B decomposition (3.4), and a rescaling of the linear map,

$$
\begin{gathered}
\mathcal{C}_{\mathcal{U}_{\mathcal{T} \mid \tilde{T}_{1}}\left(\operatorname{ev}_{\widetilde{1}} \times \mathrm{ev}_{\widehat{1}} ; \Delta_{\mathbb{P}^{3} \times \mathbb{P}^{3}}\right)=N\left(\alpha_{1}\right),} \quad \text { where } \\
\alpha_{1} \in \Gamma\left(\overline{\mathfrak{M}}_{\{\tilde{1}\} \sqcup \chi_{\tilde{1}}(\mathcal{T}) \sqcup M_{\tilde{1}} \mathcal{T}} \times \mathcal{U}_{\overline{\mathcal{T}}}(\widetilde{\mu}) ; \pi_{2}^{*} \operatorname{Hom}\left(\mathcal{F}, \operatorname{ev}_{\widehat{0}}^{*} T \mathbb{P}^{3}\right)\right), \quad \alpha_{1}(v)=\sum_{h \in \chi_{\tilde{1}}(\mathcal{T})} \mathcal{D}_{\overline{\mathcal{T}}, h}^{(1)} v_{h} .
\end{gathered}
$$

Since the linear map $\alpha_{1}$ comes entirely from the second factor, $N\left(\alpha_{1}\right)=0$ unless $\left|\chi_{\widetilde{1}}(\mathcal{T})\right|+\left|M_{\widetilde{1}} \mathcal{T}\right|=2$.

(4) Thus, we only need to consider the case $\left|\chi_{\widetilde{1}}(\mathcal{T})\right|=1$ and $M_{\tilde{1}} \mathcal{T}=\{\widehat{1}\}$ and to compute the number $N\left(\alpha_{1}\right)$, where

$$
\alpha_{1}=\mathcal{D}_{\mathcal{T}_{0}, \widetilde{1}}^{(1)} \in \Gamma\left(\mathcal{U}_{\mathcal{T}_{0}}(\widetilde{\mu}) ; \operatorname{Hom}\left(L_{\widetilde{1}}, \operatorname{ev}_{\widehat{0}}^{*} T \mathbb{P}^{3}\right)\right) .
$$


Since $\alpha_{1}$ does not vanish on $\mathcal{U}_{\mathcal{T}_{0}}(\widetilde{\mu})$ by Lemma 3.4 by Propositions 2.18A and $2.18 \mathrm{~B}$.

$$
N\left(\alpha_{1}\right)=\left\langle 6 a_{\widehat{0}}^{2}+4 a_{\widehat{0}} c_{1}\left(L_{\widetilde{1}}^{*}\right)+c_{1}^{2}\left(L_{\widetilde{1}}^{*}\right), \overline{\mathcal{U}}_{\mathcal{T}_{0}}(\widetilde{\mu})\right\rangle-\mathcal{C}_{\partial \overline{\mathcal{U}}_{\widetilde{\mathcal{T}}_{0}}(\widetilde{\mu})}\left(\alpha_{1}^{\perp}\right),
$$

where $\alpha_{1}^{\perp}$ denotes the composition of $\alpha_{1}$ with the projection $\pi_{\bar{\nu}_{1}}^{\perp}$ onto the quotient $\mathcal{O}_{1}$ of $\operatorname{ev}_{\widehat{0}}^{*} T \mathbb{P}^{3}$ by a generic trivial line subbundle $\mathbb{C} \bar{\nu}_{1}$.

(5) If $\mathcal{T}=\left(M_{0}, I ; j, \underline{d}\right)<\mathcal{T}_{0}$ is a bubble type such that $d_{\widetilde{1}}>0$, the section $\alpha_{1}^{\perp}$ does not vanish over $\mathcal{U}_{\mathcal{T} \mid \mathcal{T}_{0}}(\widetilde{\mu})$ by Lemma 3.4 if $\bar{\nu}_{1}$ is generic. Thus, $\mathcal{U}_{\mathcal{T} \mid \mathcal{T}_{0}}$ does not contribute to $\mathcal{C}_{\partial \overline{\mathcal{U}}_{\mathcal{T}_{0}}(\widetilde{\mu})}\left(\alpha_{1}^{\perp}\right)$. If $d_{\widetilde{1}}=0$, by $(3 \mathrm{~b})$ of Proposition 3.5 ,

$$
\begin{gathered}
\mathcal{D}_{\mathcal{T}_{0}, \tilde{1}}^{(1)} \phi_{\mathcal{T}_{0}, \mathcal{T}}(v)=\sum_{h \in \chi_{\tilde{1}}(\mathcal{T})}\left\{\mathcal{D}_{\mathcal{T}, h}^{(1)}+\varepsilon_{\mathcal{T}, \tilde{1} ; h}^{(1 ; 1)}(v)\right\} \rho_{\mathcal{T}, \tilde{1} ; h}^{(1 ; 1)}(v), \\
\text { where } \quad \rho_{\mathcal{T}, \widetilde{1} ; h}^{(1 ; 1)}(v)=\prod_{i \in(\widetilde{1}, h]} v_{i},
\end{gathered}
$$

for all $v \in \mathcal{F} \mathcal{T}$ sufficiently small. Thus, as before, we conclude that $\mathcal{U}_{\mathcal{T} \mid \mathcal{T}_{0}}(\widetilde{\mu})$ is $\alpha_{1}^{\perp}$-hollow unless $\widehat{I}^{+}=\chi_{\widetilde{1}}(\mathcal{T})$. If $\widehat{I}^{+}=\chi_{\widetilde{1}}(\mathcal{T})$, either $\mathcal{T}=\mathcal{T}_{0}(l)$ for some $l \in[N]$ or $\left|\widehat{I}^{+}\right|=\left|\chi_{\tilde{1}}(\mathcal{T})\right|=2$ and $\left|M_{\tilde{1}} \mathcal{T}\right|=0$. Thus, by Proposition 2.18B and decomposition (3.4),

$$
\begin{aligned}
& \mathcal{C}_{\mathcal{U}_{\mathcal{T} \mid \mathcal{T}_{0}}}\left(\alpha_{1}^{\perp}\right)=N\left(\alpha_{2}\right), \quad \text { where } \quad \alpha_{2} \in \Gamma\left(\mathcal{U}_{\overline{\mathcal{T}}}(\widetilde{\mu}) ; \operatorname{Hom}\left(\mathcal{F}_{2}, \mathcal{O}_{1}\right)\right), \\
& \mathcal{F}_{1}=\bigoplus_{h \in \chi_{\tilde{1}}(\mathcal{T})} L_{h}, \quad \mathcal{O}_{1}=\operatorname{ev}_{\widehat{0}}^{*} T \mathbb{P}^{3} / \mathbb{C} \bar{\nu}_{1}, \quad \alpha_{2}(v)=\pi_{\bar{\nu}_{1}}^{\perp} \sum_{h \in \chi_{\tilde{1}}(\mathcal{T})} \mathcal{D}_{\overline{\mathcal{T}}, h}^{(1)} v_{h} .
\end{aligned}
$$

In either case, $\alpha_{2}$ does not vanish on $\overline{\mathcal{U}}_{\overline{\mathcal{T}}}(\widetilde{\mu})$, and thus, by Propositions 2.18A and $2.18 \mathrm{~B}$.

$$
\mathcal{C}_{\partial \overline{\mathcal{U}}_{\mathcal{T}_{0}}(\widetilde{\mu})}\left(\alpha_{1}^{\perp}\right)=\sum_{l \in[N]}\left\langle 4 a_{\widehat{0}}+c_{1}\left(L_{\widetilde{1}}^{*}\right), \overline{\mathcal{U}}_{\mathcal{T}_{0} / l}(\widetilde{\mu})\right\rangle+\left|\mathcal{V}_{2}(\widetilde{\mu})\right| .
$$

The lemma follows from equations (6.1) - (6.4) and by using (3.2) and (3.3).

Lemma 6.2 With assumptions as in (3) of Lemma 1.8,

$$
\left\langle a_{\widehat{0}}^{2}, \overline{\mathcal{V}}_{1}^{(1)}(\mu)\right\rangle=2\left\langle a_{\widehat{0}}^{3}, \overline{\mathcal{V}}_{1}\left(\mu+H^{1}\right)\right\rangle-\left\langle 4 a_{\widehat{0}}^{3} \eta_{\widehat{0}, 1}+a_{\widehat{0}}^{2} \eta_{\widehat{0}, 1}^{2}, \overline{\mathcal{V}}_{1}(\mu)\right\rangle+\left\langle a_{\widehat{0}}^{2}, \overline{\mathcal{V}}_{2}(\mu)\right\rangle .
$$

Proof The proof is nearly identical to that of Lemma 6.1.

Lemma 6.3 With assumptions as in (3) of Lemma 1.8,

$$
\begin{aligned}
\left\langle a_{\widehat{0}} \eta_{\widehat{0}, 1}, \overline{\mathcal{V}}_{1}^{(1)}(\mu)\right\rangle=\left\langle a_{\widehat{0}} \eta_{\widehat{0}, 1}, \overline{\mathcal{V}}_{1}\left(\mu+H^{0}\right)\right\rangle+\left\langle a_{\widehat{0}}^{2} \eta_{\widehat{0}, 1}, \overline{\mathcal{V}}_{1}\left(\mu+H^{1}\right)\right\rangle \\
+d\left\langle a_{\widehat{0}}^{3} \eta_{\widehat{0}, 1}, \overline{\mathcal{V}}_{1}(\mu)\right\rangle-\left\langle 4 a_{\widehat{0}}^{2}+a_{\widehat{0}} \eta_{\widehat{0}, 1}, \overline{\mathcal{V}}_{2}(\mu)\right\rangle .
\end{aligned}
$$


Proof (1) Let $\widetilde{M}=M_{0} \sqcup\{\widehat{0}\}$ and let $\widetilde{\mu}$ be the $\widetilde{M}$-tuple of constraints given by $\widetilde{\mu}_{l}=\mu_{l}$ for all $l \in[N]$ and $\widetilde{\mu}_{\widehat{0}}=H^{2}$. If $s$ is a section of the bundle $\mathcal{L}_{\widetilde{1}}^{*}$ over a neighborhood of $\overline{\mathcal{U}}_{\mathcal{T}_{1}}(\widetilde{\mu})$ in $\overline{\mathcal{U}}_{\mathcal{T}_{1}}$ such that $s$ is transversal to the zero set on all smooth strata of $\mathcal{U}_{\mathcal{T}_{1}}(\mu)$, the map

$$
\mathrm{ev}_{\widetilde{1}} \times \mathrm{ev}_{\widehat{1}}: s^{-1}(0) \cap \mathcal{U}_{\mathcal{T}_{1}}(\widetilde{\mu}) \longrightarrow \mathbb{P}^{3} \times \mathbb{P}^{3}
$$

is a 6-pseudocycle. In particular, we have a well-defined intersection number,

$$
\begin{aligned}
\left\langle\left\langle a_{\widehat{0}} c_{1}\left(\mathcal{L}_{\widetilde{1}}^{*}\right), \overline{\mathcal{V}}_{1}^{(1)}(\mu)\right\rangle\right\rangle \equiv & \left\langle\left\langle s^{-1}(0) \cap \mathcal{V}_{1}^{(1)}(\widetilde{\mu})\right\rangle\right\rangle \\
\equiv & \left\langle\left\langle\left\{\operatorname{ev}_{\widetilde{1}} \times \operatorname{ev}_{\widehat{1}}\right\}^{-1}\left(\Delta_{\mathbb{P}^{3} \times \mathbb{P}^{3}}\right), s^{-1}(0) \cap \overline{\mathcal{U}}_{\mathcal{T}_{1}}(\widetilde{\mu})\right\rangle\right\rangle \\
= & \left\langle a_{\widehat{0}} c_{1}\left(\mathcal{L}_{\widetilde{1}}^{*}\right), \overline{\mathcal{V}}_{1}\left(\mu+H^{0}\right)\right\rangle+\left\langle a_{\widehat{0}}^{2} c_{1}\left(\mathcal{L}_{\widetilde{1}}^{*}\right), \overline{\mathcal{V}}_{1}\left(\mu+H^{1}\right)\right\rangle \\
& +d\left\langle a_{\widehat{0}}^{3} c_{1}\left(\mathcal{L}_{\widetilde{1}}^{*}\right), \overline{\mathcal{V}}_{1}(\mu)\right\rangle .
\end{aligned}
$$

As before,

$$
\begin{aligned}
\left\langle a_{\widehat{0}} c_{1}\left(\mathcal{L}_{\widetilde{1}}^{*}\right),\right. & \left.\overline{\mathcal{V}}_{1}^{(1)}(\mu)\right\rangle={ }^{ \pm}\left|s^{-1}(0) \cap \mathcal{V}_{1}^{(1)}(\widetilde{\mu})\right| \\
= & \left\langle\left\langle a_{\widehat{0}} c_{1}\left(\mathcal{L}_{\widetilde{1}}^{*}\right), \overline{\mathcal{V}}_{1}^{(1)}(\mu)\right\rangle\right\rangle-\mathcal{C}_{\partial \overline{\mathcal{U}}_{\mathcal{T}_{1}}(\widetilde{\mu})}\left(\mathrm{ev}_{\widetilde{1}} \times \mathrm{ev}_{\widehat{1}}, \Delta_{\mathbb{P}^{3} \times \mathbb{P}^{3}}\right),
\end{aligned}
$$

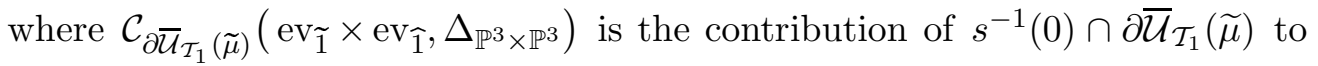
$\left\langle\left\langle s^{-1}(0) \cap \mathcal{V}_{1}^{(1)}(\widetilde{\mu})\right\rangle\right\rangle$.

(2) If $\mathcal{T}=\left(M_{1}, I ; j, \underline{d}\right)<\mathcal{T}_{1}$ is a bubble type such that $\chi_{\mathcal{T}}(\widetilde{1}, \widehat{1})>0$, the map ev $\tilde{1} \times \mathrm{ev}_{\widehat{1}}$ is transversal to $\Delta_{\mathbb{P}^{3} \times \mathbb{P}^{3}}$ on $\mathcal{U}_{\mathcal{T} \mid \mathcal{T}_{1}}(\widetilde{\mu})$ by Lemma 3.4 Thus, if $s$ is chosen to be transversal to the zero set on the set $\left\{\operatorname{ev}_{\tilde{1}} \times \operatorname{ev}_{\hat{1}}\right\}^{-1}\left(\Delta_{\mathbb{P}^{3} \times \mathbb{P}^{3}}\right) \cap$ $\mathcal{U}_{\mathcal{T} \mid \mathcal{T}_{1}}(\widetilde{\mu})$

$$
s^{-1}(0) \cap\left\{\operatorname{ev}_{\tilde{1}} \times \operatorname{ev}_{\widehat{1}}\right\}^{-1}\left(\Delta_{\mathbb{P}^{3} \times \mathbb{P}^{3}}\right) \cap \mathcal{U}_{\mathcal{T} \mid \mathcal{T}_{1}}(\widetilde{\mu})=\emptyset,
$$

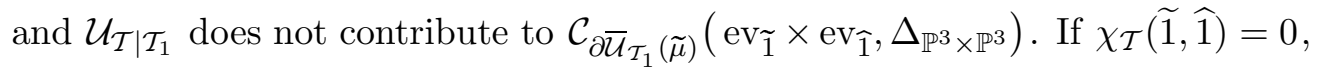
it can be assumed that $s$ is transversal to the zero set on the submanifold $\mathcal{S}_{\mathcal{T} \mid \mathcal{T}_{1}}(\mu)$, ie that $s^{-1}(0) \cap \mathcal{S}_{\mathcal{T} \mid \mathcal{T}_{1}}(\mu)=\emptyset$. Then, as in the proofs of Lemma 6.1 and 6.2 we can conclude that $s^{-1}(0) \cap \mathcal{U}_{\mathcal{T} \mid \mathcal{T}_{1}}(\mu)$ is $\left(\mathrm{ev}_{\tilde{1}} \times \mathrm{ev}_{\widehat{1}}, \Delta_{\mathbb{P}^{3} \times \mathbb{P}^{3}}\right)$-hollow unless $\chi_{\widetilde{1}}(\mathcal{T})=\widehat{I}^{+}$.

(3) We can also assume that section $\phi_{\mathcal{T}_{1}, \mathcal{T}}^{*} s$ is constant along the fibers of the bundle $\mathcal{F} \mathcal{T}$ over an open subset $\mathcal{K}_{\mathcal{T}}$ of $\mathcal{U}_{\mathcal{T} \mid \mathcal{T}_{1}}$ that contains all of the finitely many zeros of the map affine map

$$
\mathcal{F} \equiv \bigoplus_{h \in \chi_{\tilde{1}}(\mathcal{T})} L_{h} \longrightarrow \mathrm{ev}_{\widehat{0}}^{*} T \mathbb{P}^{3}, \quad(b, v) \longrightarrow \nu(b)+\sum_{h \in \chi_{\tilde{1}}(\mathcal{T})} \mathcal{D}_{\mathcal{T}, h}^{(1)} v_{h}
$$


over $s^{-1}(0) \cap \mathcal{U}_{\mathcal{T} \mid \mathcal{T}_{1}}(\mu)$ for a generic section $\nu \in \Gamma\left(\overline{\mathcal{U}}_{\mathcal{T} \mid \mathcal{T}_{1}}(\mu) ; \operatorname{ev}_{\widehat{0}}^{*} T \mathbb{P}^{3}\right)$. Then, as before,

$$
\begin{gathered}
\mathcal{C}_{\mathcal{U}_{\mathcal{T} \mid \mathcal{T}_{1}}}\left(\mathrm{ev}_{\widetilde{1}} \times \mathrm{ev}_{\widehat{1}} ; \Delta_{\mathbb{P}^{3} \times \mathbb{P}^{3}}\right)=N\left(\alpha_{1}\right), \quad \text { where } \\
\alpha_{1} \in \Gamma\left(s ^ { - 1 } ( 0 ) \cap \left(\overline{\mathfrak{M}}_{\widehat{1}, \chi_{\tilde{1}}}(\mathcal{T}), M_{\tilde{1}} \mathcal{T}\right.\right. \\
\left.\left.\alpha_{1}(v)=\sum_{h \in \chi_{\tilde{1}}(\mathcal{T})} \mathcal{U}_{\overline{\mathcal{T}}}(\widetilde{\mu})\right) ; \operatorname{Hom}\left(\mathcal{F}, \operatorname{ev}_{\widetilde{0}}^{*} T \mathbb{P}^{3}\right)\right),
\end{gathered}
$$

(4) If $c_{1}\left(\mathcal{L}_{\widetilde{1}}^{*}\right) \mid \overline{\mathcal{U}}_{\mathcal{T} \mid \mathcal{T}_{1}}(\mu)=0$, we can choose $s$ so that

$$
s^{-1}(0) \cap \overline{\mathcal{U}}_{\mathcal{T} \mid \mathcal{T}_{1}}(\mu)=\emptyset .
$$

Thus, we only need to compute contributions $N\left(\alpha_{1}\right)$ from the strata $\mathcal{U}_{\mathcal{T} \mid \mathcal{T}_{1}}(\mu)$ to which $c_{1}\left(\mathcal{L}_{\widetilde{1}}^{*}\right)$ restricts non-trivially. By dimension-counting, $\left|\chi_{\widetilde{1}}(\mathcal{T})\right| \in\{1,2\}$ if $\mathcal{U}_{\mathcal{T} \mid \mathcal{T}_{1}}(\mu) \neq \emptyset$. If $\left|\chi_{\tilde{1}}(\mathcal{T})\right|=1$, either $M_{\widetilde{1}} \mathcal{T}=\{\widehat{1}\}$ or $M_{\tilde{1}} \mathcal{T}=\{\widehat{1}, l\}$ for some $l \in[N]$. In either case, $c_{1}\left(\mathcal{L}_{\widetilde{1}}^{*}\right)$ restricts trivially to $\mathcal{U}_{\mathcal{T} \mid \mathcal{T}_{1}}(\mu)$. On the other hand, if $\left|\chi_{\widetilde{1}}(\mathcal{T})\right|=2$ and $M_{\widetilde{1}} \mathcal{T}=\{\widehat{1}, l\}$ for some $l \in[N], N\left(\alpha_{1}\right)=0$, because the second factor in the decomposition (3.4) is a finite set of points, while the map $\alpha_{1}$ comes entirely from the second factor. In the remaining case, ie $\left|\chi_{\tilde{1}}(\mathcal{T})\right|=2$ and $M_{\tilde{1}} \mathcal{T}=\{\widehat{1}\}, c_{1}\left(\mathcal{L}_{\widetilde{1}}^{*}\right)$ is the pullback of the poincare dual of a point by the projection map $\pi_{1}$. Thus, in this case,

$$
\begin{aligned}
& \mathcal{C}_{\mathcal{U}_{\mathcal{T} \mid \mathcal{T}_{1}}}\left(\mathrm{ev}_{\tilde{1}} \times \mathrm{ev}_{\widehat{1}} ; \Delta_{\mathbb{P}^{3} \times \mathbb{P}^{3}}\right)=N\left(\alpha_{1}\right), \quad \text { where } \\
& \alpha_{1} \in \Gamma\left(\mathcal{U}_{\overline{\mathcal{T}}}(\widetilde{\mu}) ; \operatorname{Hom}\left(\mathcal{F}, \mathrm{ev}_{\widehat{0}}^{*} T \mathbb{P}^{3}\right)\right), \quad \alpha_{1}(v)=\sum_{h \in \chi_{\tilde{1}}(\mathcal{T})} \mathcal{D}_{\overline{\mathcal{T}}, h}^{(1)} v_{h} .
\end{aligned}
$$

Since $\alpha_{1}$ has full rank on all of $\mathcal{U}_{\mathcal{T} \mid \mathcal{T}_{1}}(\widetilde{\mu})$, by Propositions 2.18A and 2.18B

$$
N\left(\alpha_{1}\right)=\left\langle 4 a_{\widehat{0}}+\left(c_{1}\left(L_{h_{1}}^{*}\right)+c_{1}\left(L_{h_{2}}^{*}\right)\right), \overline{\mathcal{U}}_{\overline{\mathcal{T}}}(\widetilde{\mu})\right\rangle-\mathcal{C}_{\mathbb{P} \mathcal{F} \mid \partial \overline{\mathcal{U}}_{\overline{\mathcal{T}}}(\widetilde{\mu})}\left(\widetilde{\alpha}_{1}^{\perp}\right),
$$

where $\widetilde{\alpha}_{1}^{\perp}$ denotes the composition of the linear map

$$
\widetilde{\alpha}_{1} \in \Gamma\left(\mathbb{P} \mathcal{F} ; \operatorname{Hom}\left(\gamma_{\mathcal{F}}, \pi_{\mathbb{P} \mathcal{F}}^{*} \operatorname{ev}_{\widehat{0}}^{*} T \mathbb{P}^{3}\right)\right)
$$

with the projection onto the quotient of $\pi_{\mathbb{P} \mathcal{F}}^{*} \mathrm{ev}_{\widehat{0}}^{*} T \mathbb{P}^{3}$ by a generic trivial line subbundle $\mathbb{C} \bar{\nu}_{1}$. If

$$
\mathcal{T}^{\prime} \equiv\left(M_{0}, I^{\prime} ; j^{\prime}, \underline{d}^{\prime}\right)<\overline{\mathcal{T}}
$$

is a bubble type such that $\widetilde{\alpha}_{1}$ vanishes somewhere on $\mathbb{P} \mathcal{F} \mid \mathcal{U}_{\mathcal{T}^{\prime} \mid \overline{\mathcal{T}}}(\widetilde{\mu}), \mathcal{T}^{\prime}=\overline{\mathcal{T}}(l)$ for some $l \in[N]$ and

$$
\widetilde{\alpha}_{1}^{-1}(0) \cap \mathbb{P} \mathcal{F} \mid \mathcal{U}_{\mathcal{T}^{\prime} \mid \overline{\mathcal{T}}}(\widetilde{\mu})=\left\{\left(b,\left[v_{h_{1}}, v_{h_{2}}\right]\right): b \in \mathcal{U}_{\mathcal{T}^{\prime} \mid \overline{\mathcal{T}}}(\widetilde{\mu}), v_{j_{l}^{\prime}}=0\right\},
$$


as can be seen from Lemma 3.4. From Proposition 3.5 we then conclude that

$$
\mathcal{C}_{\mathbb{P} \mathcal{F} \mid \mathcal{U}_{\mathcal{T}^{\prime} \mid \overline{\mathcal{T}}}(\widetilde{\mu})}\left(\widetilde{\alpha}_{1}^{\perp}\right)=\left|\mathcal{U}_{\mathcal{T}^{\prime} \mid \overline{\mathcal{T}}}(\widetilde{\mu})\right| .
$$

Thus, summing equation (6.7) over all bubble type $\mathcal{T}$ and using (3.2) and (3.3), we obtain

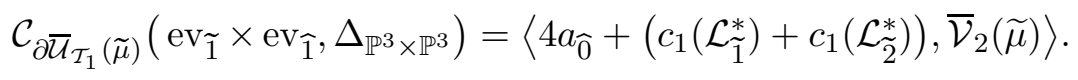

The claim follows from equations (6.5), (6.6), and (6.8).

Lemma 6.4 With assumptions as in (3) of Lemma 1.8,

$$
\begin{aligned}
\left\langle\eta_{\widehat{0}, 1}^{2}, \overline{\mathcal{V}}_{1}^{(1)}(\mu)\right\rangle=\left\langle\eta_{\widehat{0}, 1}^{2}, \overline{\mathcal{V}}_{1}(\right. & \left.\left.\mu+H^{0}\right)\right\rangle+\left\langle a_{\widehat{0}} \eta_{\widehat{0}, 1}^{2}, \overline{\mathcal{V}}_{1}\left(\mu+H^{1}\right)\right\rangle \\
+ & \left\langle 4 a_{\widehat{0}}^{3} \eta_{\widehat{0}, 1}+d \cdot a_{\widehat{0}}^{2} \eta_{\widehat{0}, 1}^{2}, \overline{\mathcal{V}}_{1}(\mu)\right\rangle-\left|\mathcal{V}_{3}(\mu)\right| .
\end{aligned}
$$

Proof (1) We proceed as in the proof of Lemma 6.3 Let $s$ be a section of $\mathcal{L}_{\widetilde{1}}^{*} \oplus \mathcal{L}_{\widetilde{1}}^{*}$ with good properties. Then, we have a well-defined homology intersection number

$$
\begin{aligned}
& \left\langle\left\langle c_{1}^{2}\left(\mathcal{L}_{\widetilde{1}}^{*}\right), \overline{\mathcal{V}}_{1}^{(1)}(\mu)\right\rangle\right\rangle \equiv\left\langle\left\langle s^{-1}(0) \cap \mathcal{V}_{1}^{(1)}(\mu)\right\rangle\right\rangle \\
& \equiv\left\langle\left\langle\left\{\operatorname{ev}_{\widetilde{1}} \times \operatorname{ev}_{\widehat{1}}\right\}^{-1}\left(\Delta_{\mathbb{P}^{3} \times \mathbb{P}^{3}}\right), s^{-1}(0) \cap \overline{\mathcal{U}}_{\mathcal{T}_{1}}(\mu)\right\rangle\right\rangle \\
& =\sum_{q+r=3}\left\langle c_{1}^{2}\left(\mathcal{L}_{\widetilde{1}}^{*}\right) a_{\widehat{0}}^{q} a_{\widehat{1}}^{r}, \overline{\mathcal{U}}_{\mathcal{I}_{1}}(\mu)\right\rangle \\
& =\left\langle c_{1}^{2}\left(\mathcal{L}_{\widetilde{1}}^{*}\right), \overline{\mathcal{V}}_{1}\left(\mu+\left\{H^{0}\right\}\right)\right\rangle+\left\langle a_{\widehat{0}} c_{1}^{2}\left(\mathcal{L}_{\widetilde{1}}^{*}\right), \overline{\mathcal{V}}_{1}\left(\mu+\left\{H^{1}\right\}\right)\right\rangle \\
& \quad+d \cdot\left\langle a_{\widetilde{1}}^{2} c_{1}^{2}\left(\mathcal{L}_{\widetilde{1}}^{*}\right), \overline{\mathcal{V}}_{1}(\mu)\right\rangle+4\left\langle a_{\widehat{0}}^{3} c_{1}\left(\mathcal{L}_{\widetilde{1}}^{*}\right), \overline{\mathcal{V}}_{1}\left(\mu+\left\{H^{0}\right\}\right)\right\rangle .
\end{aligned}
$$

A little care is required to obtain the last equality above. For example, note that

$$
\left\langle c_{1}^{2}\left(\mathcal{L}_{\widetilde{1}}^{*}\right) a_{\widehat{1}}^{3}, \overline{\mathcal{U}}_{\mathcal{T}_{1}}(\mu)\right\rangle=\left\langle c_{1}^{2}\left(\mathcal{L}_{\widetilde{1}}^{*}\right), \overline{\mathcal{V}}_{1}\left(\mu+\left\{H^{0}\right\}\right)\right\rangle+\left\langle a_{\widehat{0}}^{3} c_{1}\left(\mathcal{L}_{\widetilde{1}}^{*}\right), \overline{\mathcal{V}}_{1}\left(\mu+\left\{H^{0}\right\}\right)\right\rangle,
$$

with our definitions; see (3.2). As before,

$$
\begin{aligned}
\left\langle c_{1}^{2}\left(\mathcal{L}_{\widetilde{1}}^{*}\right), \overline{\mathcal{V}}_{1}^{(1)}(\mu)\right\rangle & ={ }^{ \pm}\left|s^{-1}(0) \cap \mathcal{V}_{1}^{(1)}(\mu)\right| \\
& =\left\langle\left\langle c_{1}^{2}\left(\mathcal{L}_{\widetilde{1}}^{*}\right), \overline{\mathcal{V}}_{1}^{(1)}(\mu)\right\rangle\right\rangle-\mathcal{C}_{\partial \overline{\mathcal{U}}_{\mathcal{T}_{1}}(\mu)}\left(\mathrm{ev}_{\widetilde{1}} \times \mathrm{ev}_{\widehat{1}}, \Delta_{\mathbb{P}^{3} \times \mathbb{P}^{3}}\right),
\end{aligned}
$$

where $\mathcal{C}_{\partial \overline{\mathcal{U}}_{\mathcal{T}_{1}(\mu)}}\left(\mathrm{ev}_{\tilde{1}} \times \mathrm{ev}_{\widehat{1}}, \Delta_{\mathbb{P}^{3} \times \mathbb{P}^{3}}\right)$ is the contribution of $s^{-1}(0) \cap \partial \overline{\mathcal{U}}_{\mathcal{T}_{1}}(\mu)$ to $\left\langle\left\langle s^{-1}(0) \cap \mathcal{V}_{1}^{(1)}(\mu)\right\rangle\right\rangle$.

(2) If $\mathcal{T}=\left(M_{1}, I ; j, \underline{d}\right)<\mathcal{T}_{1}$ is a bubble type such that $\chi_{\mathcal{T}}(\widetilde{1}, \widehat{1})>0$, as in the proof of Lemma 6.3, the space $\mathcal{U}_{\mathcal{T} \mid \mathcal{T}_{1}}$ does not contribute to

$$
\mathcal{C}_{\partial \overline{\mathcal{U}}_{\mathcal{T}_{1}}(\mu)}\left(\mathrm{ev}_{\widetilde{1}} \times \mathrm{ev}_{\widehat{1}}, \Delta_{\mathbb{P}^{3} \times \mathbb{P}^{3}}\right) .
$$


If $\chi_{\mathcal{T}}(\widetilde{1}, \widehat{1})=0$, but $\widehat{I}^{+} \neq \chi_{\widetilde{1}}(\mathcal{T}), \mathcal{U}_{\mathcal{T} \mid \mathcal{T}_{1}}(\mu)$ is $\left(\operatorname{ev}_{\widetilde{1}} \times \mathrm{ev}_{\widehat{1}}, \Delta_{\mathbb{P}^{3} \times \mathbb{P}^{3}}\right)$-hollow and again does not contribute to $\mathcal{C}_{\partial \overline{\mathcal{U}}_{\mathcal{T}_{1}}(\mu)}\left(\mathrm{ev}_{\widetilde{1}} \times \mathrm{ev}_{\widehat{1}}, \Delta_{\mathbb{P}^{3} \times \mathbb{P}^{3}}\right)$. If $\widehat{I}^{+}=\chi_{\widetilde{1}}(\mathcal{T})$, by dimension-counting

$$
\begin{gathered}
\left|\chi_{\widetilde{1}}(\mathcal{T})\right| \in\{1,2\}, \quad M_{\widetilde{1}} \mathcal{T}=\{\widehat{1}\} \quad \text { or } \quad M_{\widetilde{1}} \mathcal{T}=\{\widehat{1}, l\} \text { for some } l \in[N], \\
\text { OR } \quad\left|\chi_{\widetilde{1}}(\mathcal{T})\right|=3, \quad M_{\widetilde{1}} \mathcal{T}=\{\widehat{1}\} .
\end{gathered}
$$

In all cases, but the last, $c_{1}^{2}\left(\mathcal{L}_{\widetilde{1}}^{*}\right)$ restricts trivially to $\overline{\mathcal{U}}_{\mathcal{T} \mid \mathcal{T}_{1}}(\mu)$. If $\left|\chi_{\widetilde{1}}(\mathcal{T})\right|=3$ and $M_{\widetilde{1}} \mathcal{T}=\{\widehat{1}\}$, under the decomposition (3.4),$c_{1}^{2}\left(\mathcal{L}_{\widetilde{1}}^{*}\right)$ is the pullback of the poincare dual of a point by the projection map onto the second factor. Thus, similarly to the proof of Lemma 6.3

$$
\begin{gathered}
\mathcal{C}_{\mathcal{U}_{\mathcal{T} \mid \mathcal{T}_{1}}\left(\mathrm{ev}_{\tilde{1}} \times \mathrm{ev}_{\widehat{1}} ; \Delta_{\mathbb{P}^{3} \times \mathbb{P}^{3}}\right)=N\left(\alpha_{1}\right), \quad \text { where }} \alpha_{1} \in \Gamma\left(\mathcal{U}_{\overline{\mathcal{T}}}(\mu) ; \operatorname{Hom}\left(\mathcal{F}, \operatorname{ev}_{\widehat{0}}^{*} T \mathbb{P}^{3}\right)\right), \quad \mathcal{F}=\bigoplus_{h \in \chi_{\tilde{1}}(\mathcal{T})} L_{h}, \quad \alpha_{1}(v)=\sum_{h \in \chi_{\tilde{1}}(\mathcal{T})} \mathcal{D}_{\overline{\mathcal{T}}, h}^{(1)} v_{h} .
\end{gathered}
$$

Since $\alpha_{1}$ is an isomorphism on every fiber of $\mathcal{F}$ over the finite set $\mathcal{U}_{\mathcal{T} / M_{\tilde{1}} \mathcal{T}}(\mu)$, $N\left(\alpha_{1}\right)=\left|\mathcal{U}_{\overline{\mathcal{T}}}(\mu)\right|$. Thus, over all bubble types $\mathcal{T}$ as above, we obtain

$$
\mathcal{C}_{\partial \overline{\mathcal{U}}_{\mathcal{T}_{1}(\mu)}}\left(\mathrm{ev}_{\widetilde{1}} \times \mathrm{ev}_{\widehat{1}}, \Delta_{\mathbb{P}^{3} \times \mathbb{P}^{3}}\right)=\left|\mathcal{V}_{3}(\mu)\right| \text {. }
$$

The claim follows from equations (6.9), (6.10), and (6.11).

\subsection{Other level 1 numbers}

In this subsection, we compute the level 1 numbers of Lemmas 1.4 and 1.6 and thus conclude the computation of the enumerative numbers of Theorems 1.2 and 1.3

Lemma 6.5 With assumptions as in Lemma 1.4,

$$
\begin{aligned}
\left|\mathcal{V}_{2}^{(1)}(\mu)\right|=\mid \mathcal{V}_{2}(\mu+ & \left.H^{0}\right)\left|+\left\langle a_{\widehat{0}}, \overline{\mathcal{V}}_{2}\left(\mu+H^{1}\right)\right\rangle+3\right| \mathcal{V}_{3}(\mu) \mid \\
& -\left\langle(12-d) a_{\widehat{0}}^{2}+4 a_{\widehat{0}} \eta_{\widehat{0}, 1}+2 \eta_{\widehat{0}, 2}-\eta_{\widehat{0}, 1}^{2}, \overline{\mathcal{V}}_{2}(\mu)\right\rangle .
\end{aligned}
$$

Proof (1) By definition and the usual argument,

$$
\begin{aligned}
&\left|\mathcal{V}_{2}^{(1)}(\mu)\right|=\sum_{\tilde{\mathcal{T}}}\left(\left\langle\left\langle\left\{\mathrm{ev}_{\widetilde{1}} \times \mathrm{ev}_{\widehat{1}}\right\}^{-1}\left(\Delta_{\mathbb{P}^{3} \times \mathbb{P}^{3}}\right), \overline{\mathcal{U}}_{\widetilde{\mathcal{T}}}(\mu)\right\rangle\right\rangle\right. \\
&\left.-\mathcal{C}_{\partial \overline{\mathcal{U}}_{\tilde{\mathcal{T}}}(\mu)}\left(\mathrm{ev}_{\widetilde{1}} \times \mathrm{ev}_{\widehat{1}} ; \Delta_{\mathbb{P}^{3} \times \mathbb{P}^{3}}\right)\right) \\
&=\left|\mathcal{V}_{2}\left(\mu+H^{0}\right)\right|+\left\langle a_{\widehat{0}}, \overline{\mathcal{V}}_{2}\left(\mu+H^{1}\right)\right\rangle+d\left\langle a_{\widetilde{0}}^{2}, \overline{\mathcal{V}}_{2}(\mu)\right\rangle \\
&-\sum_{\widetilde{\mathcal{T}}} \mathcal{C}_{\partial \overline{\mathcal{U}}_{\tilde{\mathcal{T}}}(\mu)}\left(\mathrm{ev}_{\widetilde{1}} \times \mathrm{ev}_{\widehat{1}} ; \Delta_{\mathbb{P}^{3} \times \mathbb{P}^{3}}\right)
\end{aligned}
$$


where the union is taken over all bubble types $\widetilde{\mathcal{T}}=\left(M_{1}, I_{2} ; \widetilde{j}, \widetilde{d}\right)$ such that

$$
\widetilde{j}_{\widehat{1}}=\widetilde{1}, \quad \widetilde{d}_{\widetilde{1}}, \widetilde{d}_{\widetilde{2}}>0, \quad \text { and } \quad \widetilde{d}_{\widetilde{1}}+\widetilde{d}_{\widetilde{2}}=0 .
$$

Let $\mathcal{T}=\left(M_{1}, I ; j, \underline{d}\right)$ be a bubble type such that $\mathcal{T}<\widetilde{\mathcal{T}}$ and $\mathcal{U}_{\mathcal{T} \mid \widetilde{\mathcal{T}}}(\mu) \neq \emptyset$. If $\chi_{\mathcal{T}}(\widetilde{1}, \hat{1})>0$, as in the proof of Lemma 6.1, $\mathcal{U}_{\widetilde{\mathcal{T}}, \mathcal{T}}$ does not contribute to

$$
\mathcal{C}_{\partial \overline{\mathcal{U}}_{\tilde{\mathcal{T}}}(\mu)}\left(\mathrm{ev}_{\tilde{1}} \times \mathrm{ev}_{\widehat{1}} ; \Delta_{\mathbb{P}^{3} \times \mathbb{P}^{3}}\right) .
$$

Thus, we assume, $\chi_{\mathcal{T}}(\widetilde{1}, \widehat{1})=0$.

(2) By Lemma 4.2

$$
\begin{gathered}
\left\{\operatorname{ev}_{\widetilde{1}} \times \operatorname{ev}_{\widehat{1}}\right\} \phi_{\widetilde{\mathcal{T}}, \mathcal{T}}(v)=\sum_{h \in \chi_{\tilde{1}}(\mathcal{T})}\left(y_{h ; \hat{1}}-x_{\widehat{1} ; h}\right)^{-1} \otimes\left\{\mathcal{D}_{\mathcal{T}, h}^{(1)}+\varepsilon_{h}(v)\right\} \rho_{h}(v), \\
\text { where } \quad \rho_{h}(v)=\prod_{i \in\left(i_{\mathcal{T}}(h, \widehat{1}), h\right]} v_{i},
\end{gathered}
$$

for all $v \in \mathcal{F} \mathcal{T}_{\delta}$. Since the linear map,

$$
\mathcal{F} \equiv \bigoplus_{h \in \chi_{\tilde{1}}(\mathcal{T})} L_{h} \mathcal{T}, \quad v \longrightarrow \sum_{h \in \chi_{\tilde{1}}(\mathcal{T})} \mathcal{D}_{\mathcal{T}, h}^{(1)} v_{h},
$$

is injective over $\mathcal{U}_{\mathcal{T} \mid \widetilde{\mathcal{T}}}(\widetilde{\mu})$ by Lemma $3.4 \mathcal{U}_{\mathcal{T} \mid \tilde{\mathcal{T}}_{1}}$ is $\left(\mathrm{ev}_{\widetilde{1}} \times \mathrm{ev}_{\widehat{1}}, \Delta_{\mathbb{P}^{3} \times \mathbb{P}^{3}}\right)$-hollow unless $\chi_{\widetilde{1}}(\mathcal{T})=\widehat{I}^{+}$. If $\chi_{\widetilde{1}}(\mathcal{T})=\widehat{I}^{+}$by dimension-counting,

$$
\begin{aligned}
\left|\chi_{\tilde{1}}(\mathcal{T})\right|=\left|\widehat{I}^{+}\right|=1 \quad \text { and } \quad M_{\tilde{1}} \mathcal{T}=\{\widehat{1}\} \quad \text { or } \quad M_{\tilde{1}} \mathcal{T}=\{\widehat{1}, l\} \text { for some } l \in[N] \\
\text { OR } \quad\left|\chi_{\tilde{1}}(\mathcal{T})\right|=\left|\widehat{I}^{+}\right|=2 \quad \text { and } \quad M_{\tilde{1}} \mathcal{T}=\{\widehat{1}\} .
\end{aligned}
$$

Furthermore, by Proposition 2.18B a rescaling of the linear map, and the decomposition (3.4),

$$
\begin{gathered}
\mathcal{C}_{\mathcal{U}_{\mathcal{T} \mid \tilde{\mathcal{T}}}(\mu)}\left(\mathrm{ev}_{\tilde{1}} \times \mathrm{ev}_{\widehat{1}} ; \Delta_{\mathbb{P}^{3} \times \mathbb{P}^{3}}\right)=N\left(\alpha_{1}\right), \quad \text { where } \\
\alpha_{1} \in \Gamma\left(\overline{\mathfrak{M}}_{\{\widetilde{1}\} \sqcup \chi_{\tilde{1}}(\mathcal{T}) \sqcup M_{\tilde{1}}(\mathcal{T})} \times \mathcal{U}_{\overline{\mathcal{T}}}(\mu) ; \operatorname{Hom}\left(\mathcal{F}, \mathrm{ev}_{\tilde{0}}^{*} T \mathbb{P}^{3}\right)\right), \\
\mathcal{F}=\bigoplus_{h \in \chi_{\tilde{1}}(\mathcal{T})} L_{h}, \quad \alpha_{1}(v)=\sum_{h \in \chi_{\tilde{1}}(\mathcal{T})} \mathcal{D}_{\overline{\mathcal{T}}, h}^{(1)} v_{h} .
\end{gathered}
$$

Since $\alpha_{1}$ comes entirely from the second component, $N\left(\alpha_{1}\right)=0$ unless the first component is zero-dimensional, ie unless $\chi_{\tilde{1}}(\mathcal{T})=\{h\}$ is a single-element set and $M_{\tilde{1}} \mathcal{T}=\{\widehat{1}\}$. Thus, we assume that this is the case.

(3) By Propositions 2.18A and 2.18B

$$
N\left(\alpha_{1}\right)=\left\langle 6 a_{\widehat{0}}^{2}+4 a_{\widehat{0}} c_{1}\left(L_{h}^{*}\right)+c_{1}^{2}\left(L_{h}^{*}\right), \overline{\mathcal{U}}_{\overline{\mathcal{T}}}(\mu)\right\rangle-\mathcal{C}_{\partial \overline{\mathcal{U}}_{\overline{\mathcal{T}}}(\mu)}\left(\alpha_{1}^{\perp}\right) .
$$


Suppose $\mathcal{T}^{\prime}=\left(M_{0}, I^{\prime} ; j^{\prime}, \underline{d}^{\prime}\right)$ is a bubble type such that $\mathcal{T}^{\prime}<\overline{\mathcal{T}}$ and $\overline{\mathcal{U}}_{\mathcal{T}^{\prime} \mid \overline{\mathcal{T}}}(\mu) \neq$ $\emptyset$. Then, $\overline{\mathcal{U}}_{\mathcal{T}^{\prime} \mid \overline{\mathcal{T}}}(\mu)$ does not contribute to $\mathcal{C}_{\partial \overline{\mathcal{U}}_{\overline{\mathcal{T}}}(\mu)}\left(\alpha_{1}^{\perp}\right)$ unless $d_{h}^{\prime}=0$. If $d_{h}^{\prime}=0$, by Proposition 3.5.

$$
\alpha_{1} \phi_{\overline{\mathcal{T}}, \mathcal{T}^{\prime}}(v)=\sum_{h^{\prime} \in \chi_{h}\left(\mathcal{T}^{\prime}\right)}\left\{\mathcal{D}_{\mathcal{T}^{\prime}, h^{\prime}}^{(1)}+\varepsilon_{h^{\prime}}(v)\right\} \rho_{h^{\prime}}(v), \quad \text { where } \quad \rho_{h^{\prime}}(v)=\prod_{i \in\left(h, h^{\prime}\right]} v_{i},
$$

for all $v \in \mathcal{F} \mathcal{T}_{\delta}^{\prime}$. Thus, $\overline{\mathcal{U}}_{\mathcal{T}^{\prime}} \overline{\mathcal{T}}(\mu)$ is $\alpha_{1}^{\perp}$-hollow unless $\chi_{h}(\mathcal{T})=\widehat{I}^{\prime+}$. In such a case, either $\mathcal{T}^{\prime}=\overline{\mathcal{T}}(l)$ for some $l \in M_{h} \overline{\mathcal{T}}$ or $\left|\chi_{h}\left(\mathcal{T}^{\prime}\right)\right|=\left|\widehat{I}^{+}\right|=2$ and $M_{h} \mathcal{T}^{\prime}=\emptyset$. In either case,

$\mathcal{C}_{\mathcal{U}_{\mathcal{T}^{\prime} \mid \bar{T}}(\mu)}\left(\alpha_{1}^{\perp}\right)=N\left(\alpha_{1}\right), \quad$ where $\quad \alpha_{2} \in \Gamma\left(\overline{\mathcal{U}}_{\mathcal{T}^{\prime}}(\mu) ; \operatorname{Hom}\left(\mathcal{F} \mathcal{T}^{\prime}, \operatorname{ev}_{\widehat{0}}^{*} T \mathbb{P}^{3} / \mathbb{C} \bar{\nu}_{1}\right)\right)$

is a nonvanishing section. Thus, using Proposition 2.18A along with identities (3.2) and (3.2), we conclude that

$$
N\left(\alpha_{1}\right)=\left\langle 6 a_{\widehat{0}}^{2}+4 a_{\widehat{0}} c_{1}\left(\mathcal{L}_{h}^{*}\right)+c_{1}^{2}\left(\mathcal{L}_{h}^{*}\right), \overline{\mathcal{U}}_{\overline{\mathcal{T}}}(\mu)\right\rangle-\sum_{\left|\chi_{h}\left(\mathcal{T}^{\prime}\right)\right|=2}\left|\mathcal{U}_{\mathcal{T}^{\prime} \mid \overline{\mathcal{T}}}(\mu)\right| .
$$

Summing equation (6.13) over all bubble types $\tilde{\mathcal{T}}$, we obtain

$$
\begin{aligned}
\sum_{\tilde{\mathcal{T}}} \mathcal{C}_{\partial \overline{\mathcal{U}}_{\tilde{\mathcal{T}}}(\mu)}\left(\mathrm{ev}_{\widetilde{1}} \times \mathrm{ev}_{\widehat{1}} ; \Delta_{\mathbb{P}^{3} \times \mathbb{P}^{3}}\right) & \\
& =\left\langle 12 a_{\widehat{0}}^{2}+4 a_{\widehat{0}} \eta_{\widehat{0}, 1}+2 \eta_{\widehat{0}, 2}-\eta_{\widehat{0}, 1}^{2}, \overline{\mathcal{V}}_{2}(\mu)\right\rangle-3\left|\mathcal{V}_{3}(\mu)\right|
\end{aligned}
$$

The claim follows from (6.12) and (6.14).

Lemma 6.6 With assumptions as in Lemma 1.6.

$$
\left\langle a_{\widehat{0}}, \overline{\mathcal{V}}_{2}^{(1,1)}(\mu)\right\rangle=2\left\langle a_{\widehat{0}}, \overline{\mathcal{V}}_{2}\left(\mu+\left\{H^{1}: H^{2}\right\}\right)\right\rangle-\left\langle 8 a_{\widehat{0}}^{2}+2 a_{\widehat{0}} \eta_{\widehat{0}, 1}, \overline{\mathcal{V}}_{2}(\mu)\right\rangle .
$$

Proof (1) Let $\widetilde{M}=M_{0} \sqcup\{\widehat{0}\}$ and let $\widetilde{\mu}$ be the $\widetilde{M}$-tuple of constraints given by $\widetilde{\mu}_{l}=\mu_{l}$ if $l \in M_{0}$ and $\widetilde{\mu}_{\hat{0}}=H^{2}$, where $H^{2}$ is a generic hyperplane in $\mathbb{P}^{3}$. By definition and the same argument as before,

$$
\begin{array}{r}
\left\langle a_{\widehat{0}}, \overline{\mathcal{V}}_{2}^{(1,1)}(\mu)\right\rangle=\sum_{\widetilde{\mathcal{T}}}\left(\left\langle\left\langle\left\{\mathrm{ev}_{\widehat{1}} \times \mathrm{ev}_{\widehat{2}}\right\}^{-1}\left(\Delta_{\mathbb{P}^{3} \times \mathbb{P}^{3}}\right), \overline{\mathcal{U}}_{\widetilde{\mathcal{T}}}(\widetilde{\mu})\right\rangle\right\rangle\right. \\
\left.-\mathcal{C}_{\partial \overline{\mathcal{U}}_{\tilde{\mathcal{T}}}(\widetilde{\mu})}\left(\mathrm{ev}_{\widehat{1}} \times \mathrm{ev}_{\widehat{2}} ; \Delta_{\mathbb{P}^{3} \times \mathbb{P}^{3}}\right)\right) \\
=2\left\langle a_{\widehat{0}}, \overline{\mathcal{V}}_{2}\left(\mu+\left\{H^{1}: H^{2}\right\}\right)\right\rangle-\sum_{\widetilde{\mathcal{T}}} \mathcal{C}_{\partial \overline{\mathcal{U}}_{\widetilde{\mathcal{T}}}(\widetilde{\mu})}\left(\mathrm{ev}_{\widehat{1}} \times \mathrm{ev}_{\widehat{2}} ; \Delta_{\mathbb{P}^{3} \times \mathbb{P}^{3}}\right),
\end{array}
$$


where the union is taken over all bubble types $\widetilde{\mathcal{T}}=\left(M_{2}, I_{2} ; \widetilde{j}, \widetilde{d}\right)$ such that

$$
\widetilde{j}_{\widehat{1}}=\widetilde{1}, \quad \widetilde{j}_{\widehat{2}}=\widetilde{2}, \quad \widetilde{d}_{\tilde{1}}, \widetilde{d}_{\widetilde{2}}>0, \quad \text { and } \quad \widetilde{d}_{\widetilde{1}}+\widetilde{d}_{\widetilde{2}}=0 .
$$

Let $\mathcal{T}=\left(M_{2}, I ; j, \underline{d}\right)$ be a bubble type such that $\mathcal{T}<\widetilde{\mathcal{T}}$ and $\mathcal{U}_{\mathcal{T} \mid \widetilde{\mathcal{T}}}(\mu) \neq \emptyset$. If $d_{h} \neq 0$ for some $h \in \widehat{I}$ such that $h \leq j_{\widehat{1}}$ or $h \leq j_{\widehat{2}}$ as in the proof of Lemma 6.1. $\mathcal{U}_{\widetilde{\mathcal{T}}, \mathcal{T}}$ does not contribute to $\mathcal{C}_{\partial \overline{\mathcal{U}}_{\tilde{\mathcal{T}}}(\widetilde{\mu})}\left(\mathrm{ev}_{\widehat{1}} \times \mathrm{ev}_{\widehat{2}} ; \Delta_{\mathbb{P}^{3} \times \mathbb{P}^{3}}\right)$; see also Lemma 4.3 Thus, we assume that $d_{h}=0$ for all $h \in \widehat{I}$ such that $h \leq j_{\widehat{1}}$ or $h \leq j_{\widehat{2}}$.

(2) By Lemma 4.3.

$$
\begin{aligned}
\left\{\operatorname{ev}_{\widehat{1}}\right. & \left.\times \operatorname{ev}_{\widehat{2}}\right\} \phi_{\widetilde{\mathcal{T}}, \mathcal{T}}(v) \\
& =\sum_{i=1,2}(-1)^{i} \sum_{k=1}^{\infty} \sum_{h \in \chi_{\tilde{i}}(\mathcal{T})}\left(y_{h ; \hat{i}}(v)-x_{\widehat{i} ; h}(v)\right)^{-k}\left\{\mathcal{D}_{\mathcal{T}, h}^{(k)}+\varepsilon_{\mathcal{T}, \hat{i} ; h}^{(k)}(v)\right\} \rho_{\mathcal{T}, \hat{i} ; h}^{(0 ; k)}(v)
\end{aligned}
$$

for all $v \in \mathcal{F} \mathcal{T}_{\delta}-Y\left(\mathcal{F T} ; \widehat{I}^{+}\right)$. Since the map

$$
\mathcal{F} \equiv \bigoplus_{h \in \chi_{\tilde{1}}(\mathcal{T}) \cup \chi_{\tilde{2}}(\mathcal{T})} L_{h} \longrightarrow \operatorname{ev}_{\widehat{0}}^{*} T \mathbb{P}^{3}, \quad v \longrightarrow \sum_{h \in \chi_{\tilde{1}}(\mathcal{T}) \cup \chi_{\tilde{2}}(\mathcal{T})} \mathcal{D}_{\mathcal{T}, h}^{(1)} v_{h}
$$

is injective over $\mathcal{U}_{\mathcal{T} \mid \widetilde{\mathcal{T}}}(\mu)$, it follows that $\mathcal{U}_{\mathcal{T} \mid \widetilde{\mathcal{T}}}(\mu)$ is $\left(\mathrm{ev}_{\widehat{1}} \times \mathrm{ev}_{\widehat{2}}, \Delta_{\mathbb{P}^{3} \times \mathbb{P}^{3}}\right)$-hollow unless

$$
\chi_{\widetilde{1}}(\mathcal{T}) \cup \chi_{\widetilde{2}}(\mathcal{T})=\widehat{I}^{+} .
$$

In such a case, by Proposition 2.18B] a rescaling of the linear map, and the decomposition (3.4),

$$
\begin{gathered}
\mathcal{C}_{\mathcal{U}_{\mathcal{T} \mid \tilde{\mathcal{T}}}(\widetilde{\mu})}\left(\operatorname{ev}_{\widehat{1}} \times \operatorname{ev}_{\widehat{2}} ; \Delta_{\mathbb{P}^{3} \times \mathbb{P}^{3}}\right)=N\left(\alpha_{1}\right), \quad \text { where } \\
\alpha_{1} \in \Gamma\left(\overline{\mathfrak{M}}_{\{\tilde{1}\} \sqcup \chi_{\tilde{1}}(\mathcal{T}) \sqcup M_{\tilde{1}}(\mathcal{T})} \times \overline{\mathfrak{M}}_{\{\widetilde{2}\} \sqcup \chi_{\tilde{2}}(\mathcal{T}) \sqcup M_{\tilde{2}}(\mathcal{T})} \times \mathcal{U}_{\overline{\mathcal{T}}}(\widetilde{\mu}) ; \operatorname{Hom}\left(\mathcal{F}, \operatorname{ev}_{\tilde{0}}^{*} T \mathbb{P}^{3}\right)\right), \\
\alpha(v)=\sum_{h \in \chi_{\tilde{1}}(\mathcal{T}) \cup \chi_{\tilde{2}}(\mathcal{T})} \mathcal{D}_{\overline{\mathcal{T}}, h}^{(1)} v_{h} .
\end{gathered}
$$

Since $\alpha_{1}$ comes entirely from the third component, $N\left(\alpha_{1}\right)=0$ unless the first two components are zero-dimensional, ie unless

$$
\left|\chi_{\widetilde{1}}(\mathcal{T})\right|=\left|\chi_{\widetilde{2}}(\mathcal{T})\right|=1 \quad \text { and } \quad\left|M_{\widetilde{1}}(\mathcal{T})\right|=\left|M_{\widetilde{2}}(\mathcal{T})\right|=1 .
$$

It follows that

$$
\begin{gathered}
\sum_{\widetilde{\mathcal{T}}} \mathcal{C}_{\partial \overline{\mathcal{U}}_{\tilde{\mathcal{T}}}(\widetilde{\mu})}\left(\mathrm{ev}_{\widehat{1}} \times \mathrm{ev}_{\widehat{2}} ; \Delta_{\mathbb{P}^{3} \times \mathbb{P}^{3}}\right)=2 N\left(\alpha_{1}\right), \quad \text { where } \\
\alpha_{1} \in \Gamma\left(\overline{\mathcal{V}}_{2}(\widetilde{\mu}) ; \operatorname{Hom}\left(L_{\widetilde{1}} \oplus L_{\widetilde{2}}, \operatorname{ev}_{\widehat{0}}^{*} T \mathbb{P}^{3}\right)\right),\left.\quad \alpha_{1}\right|_{\mathcal{U}_{\tilde{\mathcal{T}}}(\mu)}(v)=\mathcal{D}_{\widetilde{\mathcal{T}}, \widetilde{1}}^{(1)} v_{1}+\mathcal{D}_{\widetilde{\mathcal{T}}, \tilde{2}}^{(1)} v_{2},
\end{gathered}
$$


if $\widetilde{\mathcal{T}}=\left(M_{0}, I_{2} ; \widetilde{j}, \widetilde{d}\right)$ bubble type such that $\widetilde{d}_{\widetilde{1}}, \widetilde{d}_{\widetilde{2}}>0$ and $\widetilde{d}_{\widetilde{1}}+\widetilde{d}_{\widetilde{2}}=0$. Using Propositions 2.18A and 2.18B, we conclude that

$$
\sum_{\widetilde{\mathcal{T}}} \mathcal{C}_{\partial \overline{\mathcal{U}}_{\tilde{\mathcal{T}}}(\widetilde{\mu})}\left(\mathrm{ev}_{\widehat{1}} \times \mathrm{ev}_{\widehat{2}} ; \Delta_{\mathbb{P}^{3} \times \mathbb{P}^{3}}\right)=\left\langle 8 a_{\widehat{0}}^{2}+2 a_{\widehat{0}} \eta_{\widehat{0}, 1}, \overline{\mathcal{V}}_{2}(\mu)\right\rangle
$$

This number is in fact computed in the proof of Lemma 5.13 in [17. The claim follows from equations (6.15) and (6.16).

Lemma 6.7 With assumptions as in Lemma 1.6,

$$
\begin{aligned}
\left\langle\eta_{\widehat{0}, 1}, \overline{\mathcal{V}}_{2}^{(1,1)}(\mu)\right\rangle=\left\langle\eta_{\widehat{0}, 1}, \overline{\mathcal{V}}_{2}\left(\mu+\left\{H^{1}: H^{2}\right\}\right)\right\rangle+\left|\mathcal{V}_{2}\left(\mu+H^{0}\right)\right| \\
+2\left\langle a_{\widehat{0}}, \overline{\mathcal{V}}_{2}\left(\mu+H^{1}\right)\right\rangle+2 d\left\langle a_{\widehat{0}}^{2}, \overline{\mathcal{V}}_{2}(\mu)\right\rangle-6\left|\mathcal{V}_{3}(\mu)\right|
\end{aligned}
$$

Proof The proof is a mixture of the proof of Lemma 6.6 with the proof of Lemma 6.3

\section{Other examples}

\subsection{Rational triple-pointed curves in $\mathbb{P}^{2}$}

In this subsection, we prove Proposition 7.1, ie the $\mathbb{P}^{2}$ analogue of Theorem 1.2 The method is the same as in Section 4 , but the computation is significantly simpler, since there are many fewer boundary strata to consider. Note that the formula of Proposition 7.1 agrees with [6, Lemma 3.2] and [15, Subsection 3.2].

Figure 14 outlines the computation of the boundary contribution to the homology-intersection number $\left\langle\left\langle\mathcal{V}_{1}^{(2)}(\mu)\right\rangle\right.$. It shows all non-hollow boundary strata and the multiplicity with which the number $N(\alpha)$ of zeros of an affine map over a closure of each stratum enters into $\left\langle\left\langle\mathcal{V}_{1}^{(2)}(\mu)\right\rangle\right\rangle$. In three of the cases, the number $N(\alpha)$ is easily seen to be zero. Lemma 7.2 computes the number $N(\alpha)$ in the remaining two cases.

If $d$ is a positive integer, let $n_{d}$ denote the number of degree- $d$ rational curves that pass through $3 d-1$ points in general position in $\mathbb{P}^{2}$. Following [15], we put

$$
\begin{gathered}
A_{d} \equiv n_{d}=\left\langle a_{\widehat{0}}^{2}, \overline{\mathcal{V}}_{1}(\mu)\right\rangle \\
B_{d} \equiv-\frac{n_{d}}{d}+\frac{1}{2 d} \sum_{d_{1}+d_{2}=d}\left(\begin{array}{c}
3 d-2 \\
3 d_{1}-1
\end{array}\right) d_{1}^{2} d_{2}^{2} n_{d_{1}} n_{d_{2}}=\left\langle a_{\widehat{0}} \eta_{\widehat{0}, 1}, \overline{\mathcal{V}}_{1}(\mu)\right\rangle \\
-C_{d}=\Delta_{d} \equiv \frac{1}{2} \sum_{d_{1}+d_{2}=d}\left(\begin{array}{c}
3 d-2 \\
3 d_{1}-1
\end{array}\right) d_{1} d_{2} n_{d_{1}} n_{d_{2}}=\left|\mathcal{V}_{2}(\mu)\right|=-\left\langle\eta_{\widehat{0}, 1}^{2}, \overline{\mathcal{V}}_{1}(\mu)\right\rangle,
\end{gathered}
$$


where $\mu$ is a tuple of $3 d-2$ points in $\mathbb{P}^{2}$. The computation of the above intersection numbers, with essentially the same notation as in this paper, can be found in Subsection 5.7 of [17].

Proposition 7.1 If $d$ is a positive integer, the number of rational one-component degree- $d$ curves that have a triple point and pass through a tuple $\mu$ of $3 d-2$ points in general position in $\mathbb{P}^{2}$ is $\frac{1}{6}\left|\mathcal{V}_{1}^{(2)}(\mu)\right|$, where

$$
\left|\mathcal{V}_{1}^{(2)}(\mu)\right|=3\left(d^{2}-6 d+10\right) A_{d}-3(d-6) B_{d}+6 C_{d} .
$$

Proof We use the same notation as in Section 4 except now all the stable maps under consideration have values in $\mathbb{P}^{2}$, instead of $\mathbb{P}^{3}$. Similarly to Subsection 4.1, we have

$$
\begin{aligned}
\left|\mathcal{V}_{1}^{(2)}(\mu)\right| & =\left\langle\left\langle\mathcal{V}_{1}^{(2)}(\mu)\right\rangle\right\rangle-\mathcal{C}_{\partial \overline{\mathcal{U}}_{\mathcal{T}_{2}}(\mu)}\left(\mathrm{ev}_{\tilde{1}} \times \mathrm{ev}_{\widehat{2}} ; \Delta_{\mathbb{P}^{2} \times \mathbb{P}^{2}}\right) \\
& =2 n_{d}^{(1)}+d\left\langle a_{\widehat{0}}, \overline{\mathcal{V}}_{1}^{(1)}(\mu)\right\rangle-\mathcal{C}_{\partial \overline{\mathcal{U}}_{\mathcal{T}_{2}}(\mu)}\left(\mathrm{ev}_{\widetilde{1}} \times \mathrm{ev}_{\widehat{2}} ; \Delta_{\mathbb{P}^{2} \times \mathbb{P}^{2}}\right)
\end{aligned}
$$

where $n_{d}^{(1)}$ denotes the number of degree- $d$ rational curves that pass $3 d-1$ points in general position in $\mathbb{P}^{2}$ counted with a choice of a node, ie

$$
n_{d}^{(1)}=\left(\begin{array}{c}
d-1 \\
2
\end{array}\right) n_{d}
$$

The number $\left\langle a_{\widehat{0}}, \overline{\mathcal{V}}_{1}^{(1)}(\mu)\right\rangle$ is computed in Lemma 7.5] In order to compute the boundary contribution $\mathcal{C}_{\partial \overline{\mathcal{U}}_{\mathcal{T}_{2}}(\mu)}\left(\mathrm{ev}_{\tilde{1}} \times \mathrm{ev}_{\widehat{2}} ; \Delta_{\mathbb{P}^{2} \times \mathbb{P}^{2}}\right)$, by Lemma 4.4 it is sufficient to consider only bubble types $\mathcal{T}=\left(M_{2}, I ; j, \underline{d}\right)$ such that $\mathcal{T}<\mathcal{T}_{2}$ and either $\chi_{\mathcal{T}}(\widetilde{1}, \widehat{2})=0$ or $\chi_{\mathcal{T}}(\widehat{1}, \widehat{2})=0$. Thus, the number

$$
\mathcal{C}_{\partial \overline{\mathcal{U}}_{\mathcal{T}_{2}}(\mu)}\left(\mathrm{ev}_{\widetilde{1}} \times \mathrm{ev}_{\widehat{2}} ; \Delta_{\mathbb{P}^{2} \times \mathbb{P}^{2}}\right)
$$

is computed by Lemmas 7.2 and [7.4. Finally, the numbers $\left\langle\eta_{\widehat{0}, 1}, \overline{\mathcal{V}}_{1}^{(1)}(\mu)\right\rangle$ and $\left|\mathcal{S}_{1}(\mu)\right|$ are given by Lemmas 7.5 and 7.6 .

Lemma 7.2 The total contribution to the number

$$
\mathcal{C}_{\partial \overline{\mathcal{U}}_{\mathcal{T}_{2}}(\mu)}\left(\mathrm{ev}_{\tilde{1}} \times \mathrm{ev}_{\widehat{2}} ; \Delta_{\mathbb{P}^{2} \times \mathbb{P}^{2}}\right)
$$

from the boundary strata $\mathcal{U}_{\mathcal{T}_{2}, \mathcal{T}}$, where $\mathcal{T}=\left(M_{2}, I ; j, \underline{d}\right)$ is a bubble type such that $\mathcal{T}<\mathcal{T}_{2}$ and either $\chi_{\mathcal{T}}(\widetilde{1}, \widehat{2})=0$ or $\chi_{\mathcal{T}}(\widehat{1}, \widehat{2})=0$, but not both, is given by $\sum_{\chi_{\mathcal{T}}(\tilde{1}, \widehat{2})+\chi_{\mathcal{T}}(\hat{1}, \widehat{2})>0} \mathcal{C}_{\mathcal{U}_{\mathcal{T}_{2}, \mathcal{T}}}\left(\mathrm{ev}_{\tilde{1}} \times \mathrm{ev}_{\widehat{2}} ; \Delta_{\mathbb{P}^{2} \times \mathbb{P}^{2}}\right)=\left\langle 6 a_{\widehat{0}}+2 \eta_{\widehat{0}, 1}, \overline{\mathcal{V}}_{1}^{(1)}(\mu)\right\rangle-4\left|\mathcal{S}_{1}(\mu)\right|$. 


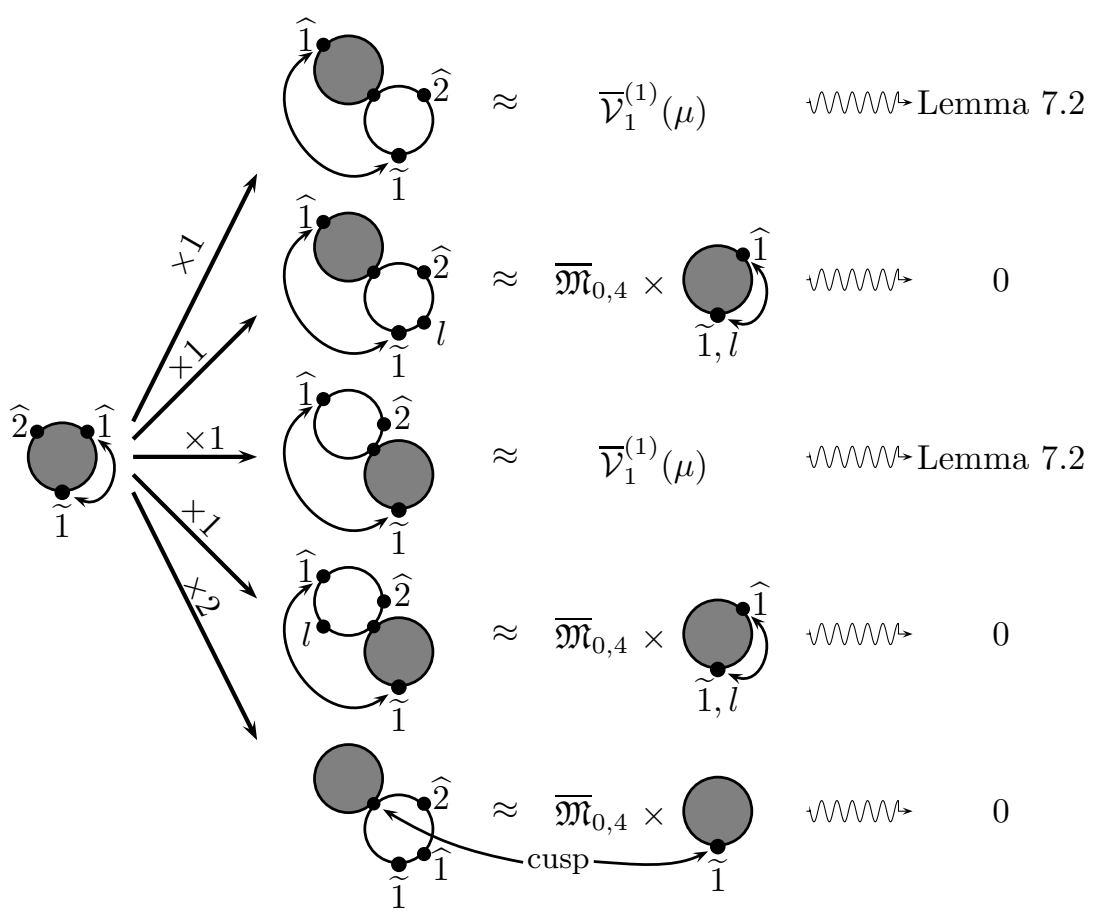

Figure 14: An outline of the proof of Proposition 7.1

Proof (1) By symmetry, it is sufficient to consider the case $\chi_{\mathcal{T}}(\widetilde{1}, \widehat{2})=0$ and $\chi_{\mathcal{T}}(\widehat{1}, \widehat{2})>0$ and then double the answer. By Lemma 4.1

$$
\overline{\mathcal{U}}_{\mathcal{T}_{2}}^{(1)}(\mu) \cap \mathcal{U}_{\mathcal{T}_{2}, \mathcal{T}} \subset \mathcal{U}_{\mathcal{T} \mid \mathcal{T}_{2}}^{(1)}(\mu)
$$

By Lemma 4.2 ,

$$
\begin{gathered}
\left\{\mathrm{ev}_{\widetilde{1}} \times \mathrm{ev}_{\widehat{2}}\right\} \phi_{\mathcal{T}_{2}, \mathcal{T}}(\mu) \sum_{h \in \chi_{\widehat{1}}(\mathcal{T})}\left(y_{h ; \widehat{2}}-x_{h ; \widehat{2}}\right)^{-1}\left\{\mathcal{D}_{\mathcal{T}, h}^{(1)}+\varepsilon_{h}(v)\right\} \rho_{h}(v), \\
\text { where } \quad \rho_{h}(v)=\prod_{i \in\left(i_{\mathcal{T}}(h, \widehat{2}), h\right]} v_{i},
\end{gathered}
$$

for all $v \in \mathcal{F} \mathcal{T}_{\delta^{*}}-Y\left(\mathcal{F} \mathcal{T} ; \widehat{I}^{+}\right)$and some $C^{0}$-negligible maps

$$
\varepsilon_{h}: \mathcal{F} \mathcal{T}_{\delta^{*}}-Y\left(\mathcal{F} \mathcal{T} ; \widehat{I}^{+}\right) \longrightarrow \operatorname{Hom}\left(L_{h}, \mathrm{ev}_{\widehat{0}}^{*} T \mathbb{P}^{2}\right) .
$$

The linear map

$$
\alpha: \mathcal{F} \equiv \bigoplus_{h \in \chi_{\tilde{1}}(\mathcal{T})} L_{h} \longrightarrow \mathrm{ev}_{\widehat{0}}^{*} T \mathbb{P}^{2}
$$


is injective over $\mathcal{U}_{\mathcal{T} \mid \mathcal{T}_{2}}^{(1)}(\mu)$ by Lemma 3.4 Thus, if $\widehat{I}^{+} \neq \chi_{\widetilde{1}}(\mathcal{T})$, then $\mathcal{U}_{\mathcal{T} \mid \mathcal{T}_{2}}^{(1)}(\mu)$ is $\left(\mathrm{ev}_{\tilde{1}} \times \mathrm{ev}_{\widehat{2}}, \Delta_{\mathbb{P}^{2} \times \mathbb{P}^{2}}\right)$-hollow, and

$$
\mathcal{C}_{\mathcal{U}_{\mathcal{T}_{2}, \mathcal{T}}}\left(\mathrm{ev}_{\widetilde{1}} \times \mathrm{ev}_{\widehat{2}} ; \Delta_{\mathbb{P}^{2} \times \mathbb{P}^{2}}\right)=0
$$

by Proposition 2.18B, or Lemma 2.20, and Lemma 4.1]

(2) On the other hand, if $\widehat{I}^{+}=\chi_{\tilde{1}}(\mathcal{T})$, by the above and Lemma 4.5, $\mathcal{U}_{\mathcal{T} \mid \mathcal{T}_{2}}^{(1)}(\mu)$ is $\left(\mathrm{ev}_{\tilde{1}} \times \mathrm{ev}_{\widehat{2}}, \Delta_{\mathbb{P}^{2} \times \mathbb{P}^{2}}\right)$-regular, and by Proposition 2.18B, a rescaling of the linear map, and the splitting (3.4),

$$
\begin{gathered}
\mathcal{C}_{\mathcal{U}_{\mathcal{T}_{2}, \mathcal{T}}}\left(\operatorname{ev}_{\widetilde{1}} \times \operatorname{ev}_{\widehat{2}} ; \Delta_{\mathbb{P}^{2} \times \mathbb{P}^{2}}\right)=N(\alpha), \quad \text { where } \\
\alpha \in \Gamma\left(\bar{M}_{\{\widetilde{1}\} \sqcup \chi_{\tilde{1}}(\mathcal{T}) \sqcup M_{\tilde{1}}(\mathcal{T})} \times \mathcal{U}_{\overline{\mathcal{T}}}^{(1)}(\mu) ; \operatorname{Hom}\left(\mathcal{F}, \operatorname{ev}_{\widehat{0}}^{*} T \mathbb{P}^{2}\right)\right), \quad \alpha(v)=\sum_{h \in \chi_{\tilde{1}}(\mathcal{T})} \mathcal{D}_{\mathcal{T}, h}^{(1)} v_{h} .
\end{gathered}
$$

Since the linear map $\alpha$ comes entirely from the second component $N(\alpha)=0$ unless the first component is zero-dimensional, ie $\left|\chi_{\widetilde{1}}(\mathcal{T})\right|=1$ and $M_{\widetilde{1}} \mathcal{T}=\{\widehat{2}\}$. Thus, we conclude that

$$
\begin{aligned}
& \sum_{\chi_{\mathcal{T}}(\widetilde{1}, \widehat{2})+\chi_{\mathcal{T}}(\widehat{1}, \widehat{2})>1} \mathcal{C}_{\mathcal{U}_{\mathcal{T}_{2}, \mathcal{T}}}\left(\mathrm{ev}_{\widetilde{1}} \times \mathrm{ev}_{\widehat{2}} ; \Delta_{\mathbb{P}^{2} \times \mathbb{P}^{2}}\right)=2 N\left(\alpha_{1}\right), \\
& \text { where } \quad \alpha_{1}=\mathcal{D}_{\mathcal{T}_{1}, \tilde{1}}^{(1)} \in \Gamma\left(\overline{\mathcal{V}}_{1}^{(1)}(\mu) ; \operatorname{Hom}\left(L_{\widetilde{1}}, \operatorname{ev}_{\widehat{0}}^{*} T \mathbb{P}^{2}\right)\right) .
\end{aligned}
$$

The number $N\left(\alpha_{1}\right)$ is computed in Lemma 7.3

Lemma 7.3 If $\alpha_{1}=\mathcal{D}_{\mathcal{T}_{1}, \tilde{1}}^{(1)} \in \Gamma\left(\overline{\mathcal{V}}_{1}^{(1)}(\mu) ; \operatorname{Hom}\left(L_{\tilde{1}}, \operatorname{ev}_{\widehat{0}}^{*} T \mathbb{P}^{2}\right)\right)$,

$$
N\left(\alpha_{1}\right)=\left\langle 3 a_{\widehat{0}}+c_{1}\left(\mathcal{L}_{\widetilde{1}}^{*}\right), \overline{\mathcal{V}}_{1}^{(1)}(\mu)\right\rangle-2\left|\overline{\mathcal{S}}_{1}(\mu)\right|
$$

Proof (1) Since $\alpha_{1}$ does not vanish on $\mathcal{V}_{1}^{(1)}(\mu)$ by Lemma 3.4 by Propositions $2.18 \mathrm{~A}$ and 2.18B.

$$
N\left(\alpha_{1}\right)=\left\langle 3 a_{\widehat{0}}+c_{1}\left(L_{\widetilde{1}}^{*}\right), \overline{\mathcal{V}}_{1}^{(1)}(\mu)\right\rangle-\mathcal{C}_{\partial \overline{\mathcal{V}}_{1}^{(1)}(\mu)}\left(\alpha_{1}^{\perp}\right),
$$

where $\alpha_{1}^{\perp}$ denotes the composition of $\alpha_{1}$ with the projection $\pi_{\bar{\nu}_{1}}^{\perp}$ onto the quotient $\mathcal{O}_{1}$ of $\mathrm{ev}_{\widehat{0}}^{*} T \mathbb{P}^{2}$ by a generic trivial line subbundle $\mathbb{C} \bar{\nu}_{1}$. Suppose

$$
\mathcal{T}=\left(M_{1}, I ; j, \underline{d}\right)
$$

is a bubble type such that $\mathcal{T}<\mathcal{T}_{1}$.

(2) If $\chi_{\mathcal{T}}(\widetilde{1}, \widehat{1})>0$, by Lemma 4.1

$$
\overline{\mathcal{V}}_{1}^{(1)}(\mu) \cap \mathcal{U}_{\mathcal{T}_{1}, \mathcal{T}} \subset \mathcal{U}_{\mathcal{T} \mid \mathcal{T}_{1}}^{(1)}(\mu) .
$$


If in addition $d_{\widetilde{1}}>0, \alpha_{1}$ does not vanish on $\mathcal{U}_{\mathcal{T} \mid \mathcal{T}_{1}}^{(1)}(\mu)$, and thus $\mathcal{U}_{\mathcal{T}_{1}, \mathcal{T}}$ does not contribute to $\mathcal{C}_{\partial \overline{\mathcal{V}}_{1}^{(1)}(\mu)}\left(\alpha_{1}^{\perp}\right)$. On the other hand, if $d_{\widetilde{1}}=0$ and $\mathcal{U}_{\mathcal{T} \mid \mathcal{T}_{1}}^{(1)}(\mu) \neq \emptyset$, by dimension-counting via Lemma $3.4, \chi_{\widetilde{1}}(\mathcal{T})=\{h\}$ is a single element-set and $\mathcal{T}=\mathcal{T}_{1}(l)$ for some $l \in[N]$. By Proposition 3.5.

$$
\mathcal{D}_{\mathcal{T}_{1}, \tilde{1}}^{(1)}\left(\phi_{\mathcal{T}_{1}, \mathcal{T}}(v)\right)=\left\{\mathcal{D}_{\mathcal{T}, h}^{(1)}+\varepsilon(v)\right\} v, \quad \text { for all } v \in \mathcal{F} \mathcal{T}_{\delta^{*}}-Y(\mathcal{F} \mathcal{T} ;\{h\}) .
$$

Since the section $\mathcal{D}_{\mathcal{T}, h}^{(1)}$ does not vanish over $\mathcal{U}_{\mathcal{T} \mid \mathcal{T}_{1}}^{(1)}(\mu)$ by Lemma 3.4 by Proposition 2.18B.

$$
\mathcal{C}_{\mathcal{U}_{\mathcal{T}_{1}, \mathcal{T}}}\left(\alpha_{1}^{\perp}\right)=\left|\mathcal{U}_{\overline{\mathcal{T}}}(\mu)\right|
$$

Summing up for all bubble types $\mathcal{T}=\mathcal{T}_{1}(l)$, we conclude that

$$
\sum_{\chi_{\mathcal{T}}(\tilde{1}, \widehat{1})>0} \mathcal{C}_{\mathcal{U}_{\mathcal{T}_{1}, \mathcal{T}}}\left(\alpha_{1}^{\perp}\right)=\left|\mathcal{V}_{1 ; 1}^{(1)}(\mu)\right| .
$$

(3) If $\mathcal{T}<\mathcal{T}_{1}$ and $\chi_{\mathcal{T}}(\widetilde{1}, \widehat{1})=0$, by Lemma 4.2

$$
\overline{\mathcal{V}}_{1}^{(1)}(\mu) \cap \mathcal{U}_{\mathcal{T}_{1}, \mathcal{T}} \subset \mathcal{S}_{\mathcal{T} \mid \mathcal{T}_{1}}(\mu) .
$$

If $\mathcal{S}_{\mathcal{T} \mid \mathcal{T}_{1}}(\mu) \neq \emptyset$, by dimension-counting via Lemma 3.4 $\chi_{\tilde{1}}(\mathcal{T})=\{h\}$ is a single element-set and $\mathcal{T}=\mathcal{T}_{1}(\widehat{1})$. Subtracting $\left(y_{\widehat{1}}-x_{h}\right)$ times the expansion of $\left\{\mathrm{ev}_{\widetilde{1}} \times \mathrm{ev}_{\widehat{1}}\right\} \circ \phi_{\mathcal{T}_{1}, \mathcal{T}}$ in Lemma 4.2 from the expansion of $\mathcal{D}_{\mathcal{T}_{1}, \widetilde{1}}^{(1)}$ in $(3 \mathrm{~b})$ of Proposition [3.5] we obtain

$$
\mathcal{D}_{\mathcal{T}_{1}, \tilde{1}_{1}}^{(1)} \phi_{\mathcal{T}_{1}, \mathcal{T}}(v)=-\left(y_{\widehat{1}}-x_{h}\right) \otimes\left\{\mathcal{D}_{\mathcal{T}, h}^{(2)}+\varepsilon(v)\right\} v \otimes v
$$

$$
\text { for all } v \in \mathcal{F} \mathcal{T}_{\delta} \text { such that } \phi_{\mathcal{T}_{1}, \mathcal{T}}(v) \in \mathcal{U}_{\mathcal{T}_{1}}^{(1)}(\mu)
$$

and for some $C^{0}$-negligible map

$$
\varepsilon: \mathcal{F} \mathcal{T}_{\delta}-Y\left(\mathcal{F} \mathcal{T} ; \widehat{I}^{+}\right) \longrightarrow L_{h}^{* \otimes 2} \otimes \operatorname{ev}_{\widehat{0}}^{*} T \mathbb{P}^{2} .
$$

By Lemma 3.4 $\mathcal{D}_{\mathcal{T}, h}^{(2)}$ does not vanish on the finite set $\mathcal{S}_{\mathcal{T} \mid \mathcal{T}_{1}}(\mu)$. Thus, by Proposition 2.18B.

$$
\sum_{\chi_{\mathcal{T}}(\widetilde{1}, \hat{1})=0} \mathcal{C}_{\mathcal{U}_{\mathcal{T}_{1}, \mathcal{T}}}\left(\alpha_{1}^{\perp}\right)=2\left|\mathcal{S}_{\mathcal{T}}(\mu)\right|=2\left|\mathcal{S}_{1}(\mu)\right| .
$$

The claim follows from (7.3) $-(7.5)$ along with (3.2) and (3.3).

Lemma 7.4 If $\mathcal{T}=\left(M_{2}, I ; j, \underline{d}\right)<\mathcal{T}_{2}$ is a bubble type such that $\chi_{\mathcal{T}}(\widetilde{1}, \widehat{2})=$ $\chi_{\mathcal{T}}(\widehat{1}, \widehat{2})=0$,

$$
\mathcal{C}_{\mathcal{U}_{\mathcal{T}_{2}, \mathcal{T}}}\left(\mathrm{ev}_{\widetilde{1}} \times \mathrm{ev}_{\widehat{2}} ; \Delta_{\mathbb{P}^{2} \times \mathbb{P}^{2}}\right)=0
$$


Proof Since $\chi_{\mathcal{T}}(\widetilde{1}, \widehat{1})=0$, by Lemma $4.2 \overline{\mathcal{U}}_{\mathcal{T}_{2}}^{(1)}(\mu) \cap \mathcal{U}_{\mathcal{T}_{2}, \mathcal{T}} \subset \mathcal{S}_{\mathcal{T} \mid \mathcal{T}_{2}}(\mu)$. If $\mathcal{S}_{\mathcal{T} \mid \mathcal{T}_{2}}(\mu) \neq \emptyset, \chi_{\widetilde{1}}(\mathcal{T})=\{h\}$ is a single-element set. Subtracting the expansion of $\left\{\mathrm{ev}_{\tilde{1}} \times \mathrm{ev}_{\widehat{1}}\right\} \circ \phi_{\mathcal{T}_{2}, \mathcal{T}}$ of Lemma 4.2 times

$$
\left(y_{h ; \widehat{1}}(v)-x_{\widehat{1} ; h}(v)\right)\left(y_{h ; \widehat{2}}(v)-x_{\widehat{2} ; h}(v)\right)
$$

from the corresponding expansion $\left\{\mathrm{ev}_{\tilde{1}} \times \mathrm{ev}_{\widehat{2}}\right\} \circ \phi_{\mathcal{T}_{2}, \mathcal{T}}$, we obtain

$\left\{\operatorname{ev}_{\widetilde{1}} \times \operatorname{ev}_{\widehat{2}}\right\} \circ \phi_{\mathcal{T}_{2}, \mathcal{T}}(v)=\{\alpha+\varepsilon(v)\} \rho(v)$ for all $v \in \mathcal{F} \mathcal{T}_{\delta}$ with $\phi_{\widetilde{\mathcal{T}}_{2}, \mathcal{T}}(v) \in \mathcal{U}_{\mathcal{T}_{1}}^{(1)}(\mu)$,

where $\rho$ is a a monomials map on $\mathcal{F} \mathcal{T}$ with values in a line bundle $\widetilde{\mathcal{F}} \mathcal{T}$ and $\alpha: \widetilde{\mathcal{F}} \mathcal{T} \longrightarrow \operatorname{ev}_{\widehat{0}}^{*} T \mathbb{P}^{2}$ is a linear map. Explicitly, if $h_{1}=i_{\mathcal{T}}(h, \widehat{1})$ and $h_{2}=$ $i_{\mathcal{T}}(h, \widehat{2})$,

$$
\begin{gathered}
\rho(v)=\prod_{i \in\left(h_{1}, h\right]} v_{i} \otimes \prod_{i \in\left(h_{2}, h\right]} v_{i}, \\
\alpha(v)=\mathcal{D}_{\mathcal{T}, h}^{(2)} \otimes \begin{cases}\left(y_{h ; \widehat{2}}-x_{\widehat{2} ; h}\right)^{-2} \otimes\left(y_{h ; \widehat{1}}-x_{\widehat{1} ; h}\right)^{-1} \otimes\left(y_{h ; \widehat{2}}-y_{h ; \widehat{1}}\right), & \text { if } h_{1}=h_{2} ; \\
\left(y_{h ; \widehat{2}}-x_{\widehat{2} ; h}\right)^{-2}, & \text { if } h_{1}<h_{2} ; \\
\left(y_{h ; \widehat{2}}-x_{\widehat{2} ; h}\right)^{-1} \otimes\left(y_{h ; \widehat{1}}-x_{\widehat{1} ; h}\right)^{-1}, & \text { if } h_{1}>h_{2} .\end{cases}
\end{gathered}
$$

Thus, $\mathcal{S}_{\mathcal{T} \mid \mathcal{T}_{2}}(\mu)$ is $\left(\mathrm{ev}_{\widetilde{1}} \times \mathrm{ev}_{\widehat{2}}, \Delta_{\mathbb{P}^{3} \times \mathbb{P}^{3}}\right)$-hollow unless $\widehat{I}^{+}=\chi_{\widetilde{1}}(\mathcal{T})$. On the other hand, if $\widehat{I}^{+}=\chi_{\widetilde{1}}(\mathcal{T})$, by Proposition 2.18B the decomposition (3.4), and a rescaling of the linear map,

$$
\begin{gathered}
\mathcal{C}_{\mathcal{U}_{\mathcal{T}_{2}, \mathcal{T}}}\left(\mathrm{ev}_{\tilde{1}} \times \mathrm{ev}_{\widehat{2}} ; \Delta_{\mathbb{P}^{3} \times \mathbb{P}^{3}}\right)=2 N\left(\alpha_{1}\right), \quad \text { where } \\
\alpha_{1}=\pi_{2}^{*} \mathcal{D}_{\overline{\mathcal{T}}}^{(2)} \in \Gamma\left(\overline{\mathfrak{M}}_{\{\widetilde{1}, h\} \sqcup M_{\tilde{1}} \mathcal{T}} \times \mathcal{S}_{\overline{\mathcal{T}}}(\mu) ; \pi_{2}^{*} \operatorname{Hom}\left(L_{\widetilde{1}}^{*}, \operatorname{ev}_{\widehat{0}}^{*} T \mathbb{P}^{3}\right)\right) .
\end{gathered}
$$

Since $\left|M_{\tilde{1}} \mathcal{T}\right| \geq 2$, the first factor is positive-dimensional, while the linear map $\alpha_{1}$ comes entirely from the second factor. Thus,

$$
\mathcal{C}_{\mathcal{U}_{\mathcal{T}_{2}, \mathcal{T}}}\left(\mathrm{ev}_{\widetilde{1}} \times \mathrm{ev}_{\widehat{2}} ; \Delta_{\mathbb{P}^{3} \times \mathbb{P}^{3}}\right)=N\left(\alpha_{1}\right)=0
$$

Lemma 7.5 If $d$ is a positive integer and $\mu$ is a tuple of $3 d-2$ points in general position in $\mathbb{P}^{2}$, the number of rational one-component degree- $d$ curves that pass through the constraints $\mu$ and have a node on a generic line is $\frac{1}{2}\left\langle a_{\widehat{0}}, \bar{V}_{1}^{(1)}(\mu)\right\rangle$, where

$$
\left\langle a_{\widehat{0}}, \overline{\mathcal{V}}_{1}^{(1)}(\mu)\right\rangle=\left\langle(2 d-3) a_{\widehat{0}}^{2}-a_{\widehat{0}} \eta_{\widehat{0}, 1}, \overline{\mathcal{V}}_{1}(\mu)\right\rangle
$$

Furthermore,

$$
\left\langle\eta_{\widehat{0}, 1}, \overline{\mathcal{V}}_{1}^{(1)}(\mu)\right\rangle=\left\langle a_{\widehat{0}}^{2}+d \cdot a_{\widehat{0}} \eta_{\widehat{0}, 1}, \overline{\mathcal{V}}_{1}(\mu)\right\rangle-\left|\mathcal{V}_{2}(\mu)\right|
$$


Proof (1) In order to prove the first identity, we take $\widetilde{\mu}$ to be the $\widetilde{M} \equiv$ $[N] \cup\{\widehat{0}\}$-tuple of constraints defined by $\widetilde{\mu}_{l}=\mu_{l}$ and $\widetilde{\mu}_{\widehat{0}}=H^{1}$, where $H^{1}$ is a generic hyperplane. Similarly to the proof of Lemma 6.1

$$
\begin{aligned}
& \left\langle a_{\widehat{0}}, \overline{\mathcal{V}}_{1}^{(1)}(\mu)\right\rangle=\left\langle\left\langle a_{\widehat{0}}, \overline{\mathcal{V}}_{1}^{(1)}(\mu)\right\rangle\right\rangle-\mathcal{C}_{\partial \overline{\mathcal{U}}_{\mathcal{T}_{1}}(\widetilde{\mu})}\left(\mathrm{ev}_{\widetilde{1}} \times \mathrm{ev}_{\widehat{1}}, \Delta_{\mathbb{P}^{2} \times \mathbb{P}^{2}}\right) \\
& =2 d\left\langle a_{\widehat{0}}^{2}, \overline{\mathcal{V}}_{1}(\mu)\right\rangle-\mathcal{C}_{\partial \overline{\mathcal{U}}_{\tau_{1}}(\widetilde{\mu})}\left(\mathrm{ev}_{\widetilde{1}} \times \mathrm{ev}_{\widehat{1}}, \Delta_{\mathbb{P}^{2} \times \mathbb{P}^{2}}\right),
\end{aligned}
$$

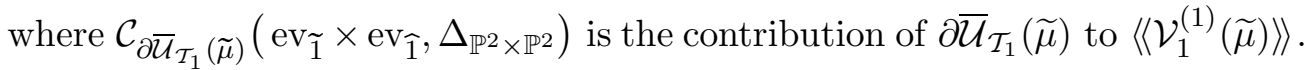
If $\mathcal{T}=\left(M_{1}, I ; j, \underline{d}\right)<\mathcal{T}_{1}$ is a bubble type such that $\chi_{\mathcal{T}}(\widetilde{1}, \widehat{1})>0$, the map $\mathrm{ev}_{\tilde{1}} \times \mathrm{ev}_{\widehat{1}}$ is transversal to $\Delta_{\mathbb{P}^{2} \times \mathbb{P}^{2}}$ on $\mathcal{U}_{\mathcal{T} \mid \mathcal{T}_{1}}(\widetilde{\mu})$ by Lemma 3.4 and thus the boundary stratum $\mathcal{U}_{\mathcal{T}_{1}, \mathcal{T}}(\widetilde{\mu})$ does not contribute to the number

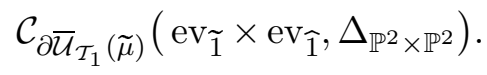

If $\chi_{\mathcal{T}}(\widetilde{1}, \widehat{1})=0$ and $\mathcal{U}_{\mathcal{T} \mid \mathcal{T}_{1}}(\widetilde{\mu}) \neq \emptyset, \chi_{\widetilde{1}}(\mathcal{T})=\{h\}$ and $M_{\widetilde{1}} \mathcal{T}=\{\widehat{1}\}$ are singleelement sets. By Lemma 4.2 .

$$
\left\{\mathrm{ev}_{\widetilde{1}} \times \mathrm{ev}_{\widehat{1}}\right\} \phi_{\mathcal{T}_{1}, \mathcal{T}}(v)=\left(y_{\widehat{1}}-x_{h}\right)^{-1} \otimes\left\{\mathcal{D}_{\mathcal{T}_{, h}}^{(1)}+\varepsilon(v)\right\} v \quad \text { for all } v \in \mathcal{F} \mathcal{T}_{\delta} .
$$

Since the section $\mathcal{D}_{\mathcal{T}, h}^{(1)}$ does not vanish on $\mathcal{U}_{\mathcal{T} \mid \mathcal{T}_{1}}(\mu)$ by Lemma 3.4. $\mathcal{U}_{\mathcal{T} \mid \mathcal{T}_{1}}$ is $\left(\mathrm{ev}_{\tilde{1}} \times \mathrm{ev}_{\widehat{1}}, \Delta_{\mathbb{P}^{2} \times \mathbb{P}^{2}}\right)$-hollow unless $\widehat{I}^{+}=\{h\}$. If $\widehat{I}^{+}=\{h\}$, by Proposition 2.18B decomposition (3.4), and a rescaling of the linear map,

$$
\begin{gathered}
\mathcal{C}_{\mathcal{U}_{\mathcal{T} \mid \tilde{\tau}_{1}}(\widetilde{\mu})}\left(\mathrm{ev}_{\widetilde{1}} \times \mathrm{ev}_{\widehat{1}} ; \Delta_{\mathbb{P}^{2} \times \mathbb{P}^{2}}\right)=N\left(\alpha_{1}\right), \quad \text { where } \\
\alpha_{1}=\mathcal{D}_{\mathcal{T}_{0}, \hat{1}}^{(1)} \in \Gamma\left(\overline{\mathcal{V}}_{1}(\widetilde{\mu}) ; \operatorname{Hom}\left(L_{\widetilde{1}}, \mathrm{ev}_{\widehat{0}}^{*} T \mathbb{P}^{2}\right)\right) .
\end{gathered}
$$

By Propositions 2.18A and 2.18B

$$
N\left(\alpha_{1}\right)=\left\langle 3 a_{\widehat{0}}+c_{1}\left(L_{\widetilde{1}}^{*}\right), \overline{\mathcal{V}}_{1}(\widetilde{\mu})\right\rangle-\mathcal{C}_{\partial \overline{\mathcal{V}}_{1}(\mu)}\left(\alpha_{1}^{\perp}\right) .
$$

If $\mathcal{T} \equiv\left(M_{0}, I ; j, \underline{d}\right)<\mathcal{T}_{0}$ is a bubble type such that $\alpha_{1}$ vanishes somewhere on $\mathcal{U}_{\mathcal{T} \mid \mathcal{T}_{0}}(\widetilde{\mu}), \mathcal{T}=\mathcal{T}_{1}(l)$ for some $l \in[N]$. From Proposition 3.5], we then obtain

$$
\mathcal{C}_{\partial \overline{\mathcal{V}}_{1}(\widetilde{\mu})}\left(\alpha_{1}^{\perp}\right)=\left|\mathcal{V}_{1 ; 1}(\widetilde{\mu})\right|
$$

Putting everything together and using identities (3.2) and (3.3), we conclude that

$$
\mathcal{C}_{\partial \overline{\mathcal{U}}_{\mathcal{T}_{1}}(\tilde{\mu})}\left(\mathrm{ev}_{\widetilde{1}} \times \mathrm{ev}_{\widehat{1}}, \Delta_{\mathbb{P}^{2} \times \mathbb{P}^{2}}\right)=\left\langle 3 a_{\widehat{0}}^{2}+a_{\widehat{0}} c_{1}\left(\mathcal{L}_{\widetilde{1}}^{*}\right), \overline{\mathcal{V}}_{1}(\mu)\right\rangle
$$

The first claim of the lemma follows from (7.6) and (7.7). 
(2) Let $s$ be a section of $\mathcal{L}_{\widetilde{1}}^{*}$ with good properties, ie as in the proof of Lemma 6.3. Then,

$$
\begin{aligned}
&\left\langle c_{1}\left(\mathcal{L}_{\widetilde{1}}^{*}\right), \overline{\mathcal{V}}_{1}^{(1)}(\mu)\right\rangle \\
&=\left\langle\left\langle c_{1}\left(\mathcal{L}_{\widetilde{1}}^{*}\right), \overline{\mathcal{V}}_{1}^{(1)}(\mu)\right\rangle\right\rangle-\mathcal{C}_{s^{-1}(0) \cap \partial \overline{\mathcal{U}}_{\tau_{1}}(\mu)}\left(\mathrm{ev}_{\widetilde{1}} \times \mathrm{ev}_{\widehat{1}}, \Delta_{\mathbb{P}^{2} \times \mathbb{P}^{2}}\right) \\
&=\left\langle c_{1}\left(\mathcal{L}_{\widetilde{1}}^{*}\right), \overline{\mathcal{V}}_{1}\left(\mu+H^{0}\right)\right\rangle+\left\langle a_{\widehat{0}} c_{1}\left(\mathcal{L}_{\widetilde{1}}^{*}\right), \overline{\mathcal{V}}_{1}\left(\mu+H^{1}\right)\right\rangle+3\left\langle a_{\widehat{0}}^{2}, \overline{\mathcal{V}}_{1}(\mu)\right\rangle \\
& \quad-\mathcal{C}_{s^{-1}(0) \cap \partial \overline{\mathcal{U}}_{\tau_{1}}(\mu)}\left(\mathrm{ev}_{\widetilde{1}} \times \mathrm{ev}_{\widehat{1}}, \Delta_{\mathbb{P}^{2} \times \mathbb{P}^{2}}\right) \\
&=\left\langle a_{\widehat{0}}^{2}+d \cdot a_{\widehat{0}} c_{1}\left(\mathcal{L}_{\widetilde{1}}^{*}\right), \overline{\mathcal{V}}_{1}\left(\mu+H^{1}\right)\right\rangle-\mathcal{C}_{s^{-1}(0) \cap \partial \overline{\mathcal{U}}_{\mathcal{T}_{1}}(\mu)}\left(\mathrm{ev}_{\widetilde{1}} \times \mathrm{ev}_{\widehat{1}}, \Delta_{\mathbb{P}^{2} \times \mathbb{P}^{2}}\right)
\end{aligned}
$$

In the last equality we used Lemma 5.17 of [17], which is essentially Lemma 2.2.2 of [1]. If

$$
\mathcal{T} \equiv\left(M_{1}, I ; j, \underline{d}\right)<\mathcal{T}_{1}
$$

is a bubble type such that $\chi_{\mathcal{T}}(\widetilde{1}, \widehat{1})>0$, the space $\mathcal{U}_{\mathcal{T} \mid \mathcal{T}_{1}}$ does not contribute to $\mathcal{C}_{\partial \overline{\mathcal{U}}_{\mathcal{T}_{1}}(\mu)}\left(\mathrm{ev}_{\widetilde{1}} \times \mathrm{ev}_{\widehat{1}}, \Delta_{\mathbb{P}^{2} \times \mathbb{P}^{2}}\right)$ unless $\chi_{\mathcal{T}}(\widetilde{1}, \widehat{1})=0$ and $\eta_{\widehat{0}, 1} \mid \overline{\mathcal{U}}_{\mathcal{T} \mid \mathcal{T}_{1}}(\mu) \neq 0$. On the other hand,

$$
\chi_{\mathcal{T}}(\widetilde{1}, \widehat{1})=0, \quad \eta_{\widehat{0}, 1} \mid \overline{\mathcal{U}}_{\mathcal{T} \mid \mathcal{T}_{1}}(\mu) \neq 0 \Longrightarrow \widehat{I}^{+}=\chi_{\widetilde{1}}(\mathcal{T})=\left\{h_{1}, h_{2}\right\}, \quad M_{\widetilde{1}}(\mathcal{T})=\{\widehat{1}\} .
$$

By Lemma 4.2,

$$
\left\{\mathrm{ev}_{\tilde{1}} \times \mathrm{ev}_{\hat{1}}\right\} \phi_{\mathcal{T}_{1}, \mathcal{T}}(v)=\sum_{h \in \chi_{\tilde{1}}(\mathcal{T})}\left(y_{\widehat{1}}-x_{h}\right)^{-1} \otimes\left\{\mathcal{D}_{\mathcal{T}, h}^{(1)}+\varepsilon_{h}(v)\right\} v_{h},
$$

for all $v \in \mathcal{F} \mathcal{T}_{\delta}$. Thus,

$$
\mathcal{C}_{\mathcal{U}_{\mathcal{T} \mid \mathcal{T}_{1}}(\mu)}\left(\mathrm{ev}_{\widetilde{1}} \times \mathrm{ev}_{\widehat{1}} ; \Delta_{\mathbb{P}^{2} \times \mathbb{P}^{2}}\right)={ }^{ \pm}\left|s^{-1}(0) \cap \mathcal{U}_{\mathcal{T} \mid \mathcal{T}_{1}}(\mu)\right|=\left|\mathcal{U}_{\overline{\mathcal{T}}}(\mu)\right|
$$

We conclude that

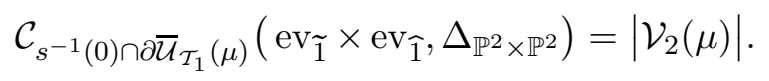

The second claim of the lemma follows from (7.8) and (7.9).

Lemma 7.6 If $d \geq 1$, the number of rational degree- $d$ cuspidal curves passing through a tuple $\mu$ of $3 d-2$ points in general position in $\mathbb{P}^{2}$ is given by

$$
\left|\mathcal{S}_{1}(\mu)\right|=\left\langle 3 a_{\widehat{0}}^{2}+3 a_{\widehat{0}} \eta_{\widehat{0}, 1}+\eta_{\widehat{0}, 1}^{2}, \overline{\mathcal{V}}_{1}(\mu)\right\rangle-\left|\mathcal{V}_{2}(\mu)\right|
$$

Proof This is the $n=2$ case of Theorem 1.1] see [17, Lemma 5.4] for a direct proof. The same formula can also be found in [11, Subsection 4.5] and [15. Subsection 3.2]. 


\subsection{Rational tacnodal curves in $\mathbb{P}^{2}$}

In this subsection, we prove Proposition [7.7, the $\mathbb{P}^{2}$-analogue of Theorem 1.3. The formula we obtain agrees with previously known results; see equation (1.2) in 2] and Subsection 3.2 in [15].

Figure 15 shows the three types of boundary strata $\overline{\mathcal{V}}_{1}^{(1)}(\mu) \cap \mathcal{U}_{\mathcal{T}_{1}, \mathcal{T}}$ such that

$$
\mathbb{P}\left(L_{\widetilde{1}} \oplus L_{\widetilde{1}}^{*}\right) \mid \overline{\mathcal{V}}_{1}^{(1)}(\mu) \cap \mathcal{U}_{\mathcal{T}_{1}, \mathcal{T}}
$$

is not contained in a finite union of $\mathcal{D}_{\widetilde{1}, \hat{1}}$-hollow sets. For such boundary strata,

$$
\mathbb{P}\left(L_{\tilde{1}} \oplus L_{\widetilde{1}}^{*}\right) \mid \overline{\mathcal{V}}_{1}^{(1)}(\mu) \cap \mathcal{U}_{\mathcal{T}_{1}, \mathcal{T}}
$$

is a union of one $\mathcal{D}_{\widetilde{1}, \hat{1}}$-regular or hollow subset and one $\mathcal{D}_{\widetilde{1}, \hat{1}}$-regular subset: a section over the base $\overline{\mathcal{V}}_{1}^{(1)}(\mu) \cap \mathcal{U}_{\mathcal{T}_{1}, \mathcal{T}}$ and its complement. The second-to-last column of Figure 15] shows the multiplicity with which each number $N(\alpha)$ of zeros of an affine map over a closure of the larger and the smaller subset, if it is regular, enters into the euler class of the bundle $\gamma_{L_{\tilde{1}} \oplus L_{\tilde{1}}^{*}}^{*} \otimes \mathrm{ev}_{\widehat{0}}^{*} T \mathbb{P}^{2}$ as computed via the section $\mathcal{D}_{\tilde{1}, \hat{1}}$. The last column gives the number $N(\alpha)$ for each regular subset of the boundary strata. Contributions from the boundary strata as in the first row of Figure 15] are computed in Lemma 7.8. Lemma [7.9 deals with the boundary strata as in the last two rows of Figure 15.

Proposition 7.7 If $d$ is a nonnegative integer, the number of rational onecomponent degree- $d$ curves that have a tacnodal point and pass through a tuple $\mu$ of $3 d-2$ points in general position in $\mathbb{P}^{2}$ is $\frac{1}{2}\left|\mathcal{S}_{1}^{(1)}(\mu)\right|$, where

$$
\left|\mathcal{S}_{1}^{(1)}(\mu)\right|=2(3 d-11) A_{d}+2(d-9) B_{d}-8 C_{d} .
$$

Proof Similarly to Subsection 5.1

$$
\left|\mathcal{S}_{1}^{(1)}(\mu)\right|=\left\langle 3 a_{\widehat{0}}, \overline{\mathcal{V}}_{1}^{(1)}(\mu)\right\rangle-\mathcal{C}_{\partial \mathbb{P}\left(L_{\tilde{1}} \oplus L_{\widetilde{1}}^{*}\right)}\left(\mathcal{D}_{\widetilde{1}, \hat{1}}\right),
$$

where $\mathcal{C}_{\partial \mathbb{P}\left(L_{\widetilde{1}} \oplus L_{\widetilde{1}}^{*}\right)}\left(\mathcal{D}_{\widetilde{1}, \hat{1}}\right)$ is the contribution from the boundary strata of the space $\mathbb{P}\left(L_{\widetilde{1}} \oplus L_{\widetilde{1}}^{*}\right)$. This contribution is computed in Lemmas 7.8 and 7.9. The numbers $\left\langle a_{\widehat{0}}, \overline{\mathcal{V}}_{1}^{(1)}(\mu)\right\rangle$ and $\left|\mathcal{S}_{1}(\mu)\right|$ are given by Lemmas 7.5 and 7.6 Finally, the number of two-component rational curves that pass through $3 d-2$ points in general position in $\mathbb{P}^{2}$, counted a with choice of an ordered pair of distinct 


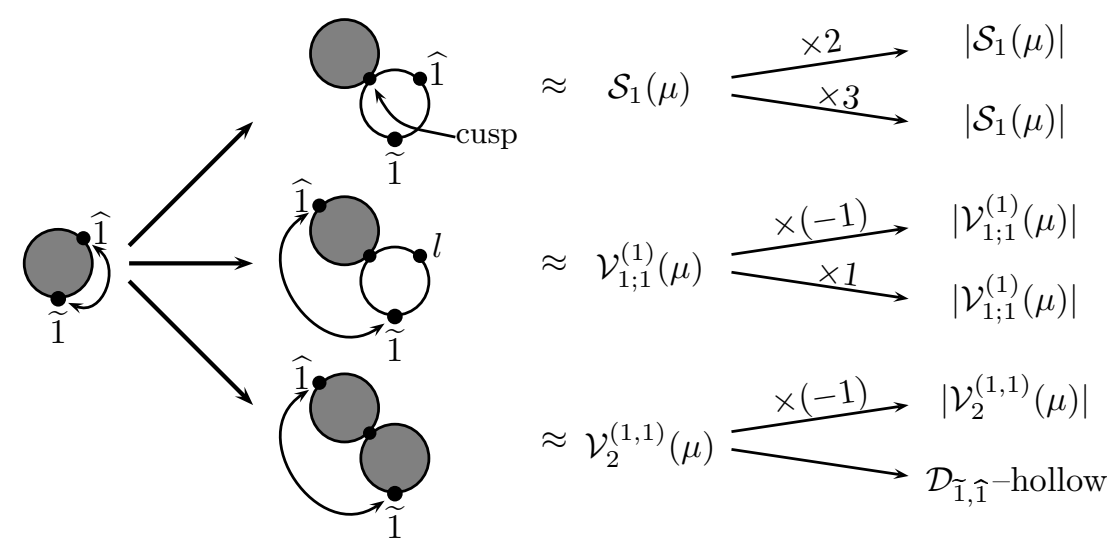

Figure 15: An outline of the proof of Proposition 7.7

nodes at which the two components intersect and with a choice of a branch at one of these nodes, is easily seen to be

$$
\begin{aligned}
\left|\mathcal{V}_{2}^{(1,1)}(\mu)\right| & =\sum_{d_{1}+d_{2}=d}\left(\begin{array}{c}
3 d-2 \\
3 d_{1}-1
\end{array}\right) d_{1} d_{2}\left(d_{1} d_{2}-1\right) n_{d_{1}} n_{d_{2}} \\
& =2 A_{d}+2 d B_{d}+2 C_{d}
\end{aligned}
$$

which proves the claim.

Lemma 7.8 The total contribution to $\mathcal{C}_{\partial \mathbb{P}\left(L_{\widetilde{1}} \oplus L_{\tilde{1}}^{*}\right)}\left(\mathcal{D}_{\widetilde{1}, \hat{1}}\right)$ from the boundary strata $\mathbb{P}\left(L_{\widetilde{1}} \oplus L_{\widetilde{1}}^{*}\right) \mid \mathcal{U}_{\mathcal{T}_{1}, \mathcal{T}}$, where $\mathcal{T}=\left(M_{1}, I ; j, \underline{d}\right)$ is a bubble type such that $\mathcal{T}<\mathcal{T}_{1}$ and $\chi_{\mathcal{T}}(\widetilde{1}, \widehat{1})=0$, is given by

$$
\sum_{\chi_{\mathcal{T}}(\widetilde{1}, \widehat{1})=0} \mathcal{C}_{\partial \mathbb{P}\left(L_{\widetilde{1}} \oplus L_{\tilde{1}}^{*}\right) \mid \mathcal{U}_{\mathcal{T}_{2}, \mathcal{T}}}\left(\mathcal{D}_{\widetilde{1}, \hat{1}}\right)=5\left|\mathcal{S}_{1}(\mu)\right| .
$$

Proof (1) By Lemma 4.2

$$
\overline{\mathcal{U}}_{\mathcal{T}_{1}}^{(1)}(\mu) \cap \mathcal{U}_{\mathcal{T}_{1}, \mathcal{T}} \subset \mathcal{S}_{\mathcal{T} \mid \mathcal{T}_{1}}(\mu)
$$

If $\mathcal{S}_{\mathcal{T} \mid \mathcal{T}_{1}}(\mu) \neq \emptyset, \widehat{I}^{+}=\chi_{\widetilde{1}}(\mathcal{T})=\{h\}$ and $M_{\widetilde{1}} \mathcal{T}=\{\widehat{1}\}$ are a single-element sets. Subtracting the expansion of $\left\{\mathrm{ev}_{\tilde{1}} \times \mathrm{ev}_{\hat{1}}\right\} \circ \phi_{\mathcal{T}_{1}, \mathcal{T}}$ of Lemma 4.2 multiplied by $\left(y_{\widehat{1}}-x_{h}\right)$ and by $-\left(y_{\widehat{1}}-x_{h}\right)^{-1}$ from the expansions of $\mathcal{D}_{\mathcal{T}_{1}, \tilde{1}}^{(1)} \circ \phi_{\mathcal{T}_{1}, \mathcal{T}}$ and 
$\mathcal{D}_{\mathcal{T}_{1}, \widehat{1}}^{(1)} \circ \phi_{\mathcal{T}_{1}, \mathcal{T}}$, respectively, given by Proposition 3.5, we obtain

$$
\begin{array}{r}
\mathcal{D}_{\tilde{1}, \hat{1}} \phi_{\mathcal{T}_{1}, \mathcal{T}}\left(\left[v_{\tilde{1}}, v_{\hat{1}}\right] ; v\right)=-\left\{\left(\left(y_{\widehat{1}}-x_{h}\right)^{-1} \otimes v_{\tilde{1}}+\left(y_{\widehat{1}}-x_{h}\right)^{-3} \otimes v_{\hat{1}}\right) \otimes \mathcal{D}_{\mathcal{T}, h}^{(2)}\right. \\
+\varepsilon(v)\} v \otimes v
\end{array}
$$

for all $\left(\left[v_{\tilde{1}}, v_{\hat{1}}\right] ; v\right) \in \mathcal{F} \mathcal{T}_{\delta}$ such that $\phi_{\mathcal{T}_{1}, \mathcal{T}}(v) \in \mathcal{U}_{\mathcal{T}_{1}}^{(1)}$. Let

$$
\mathcal{Z}_{\mathcal{T}}=\left\{\left[v_{\widetilde{1}}, v_{\widehat{1}}\right] \in \mathbb{P}\left(L_{\widetilde{1}} \oplus L_{\widetilde{1}}^{*}\right) \mid \mathcal{U}_{\mathcal{T} \mid \mathcal{T}_{1}}:\left(y_{\widehat{1}}-x_{h}\right)^{-1} v_{\widetilde{1}}+\left(y_{\widehat{1}}-x_{h}\right)^{-3} v_{\widehat{1}}=0\right\} .
$$

Since the section $\mathcal{D}_{\mathcal{T}, h}^{(2)}$ does not vanish over $\mathcal{S}_{\mathcal{T} \mid \mathcal{T}_{1}}(\mu)$, by Proposition 2.18B the decomposition (3.4), and a rescaling of the linear map,

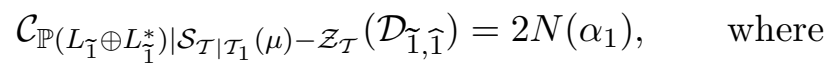

$$
\begin{aligned}
& \alpha_{1} \in \Gamma\left(\mathbb{P}^{1} \times \mathcal{S}_{1}(\mu) ; \operatorname{Hom}\left(\gamma^{*}, \gamma^{*} \otimes \operatorname{ev}_{\widehat{0}}^{*} T \mathbb{P}^{2}\right)\right)
\end{aligned}
$$

is a nonvanishing section. Thus, by Proposition $2.18 \mathrm{~A}$

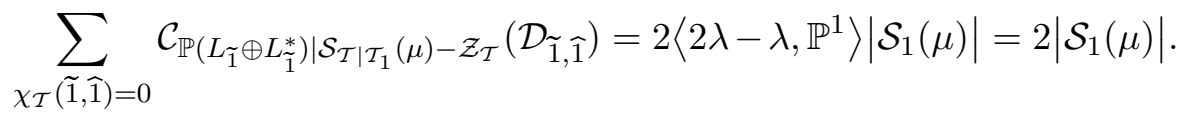

(3) In order to compute the contribution from the space $\mathcal{Z}_{\mathcal{T}}$, we model a neighborhood of $\mathcal{Z}_{\mathcal{T}}$ in $\mathbb{P}\left(L_{\tilde{1}} \oplus L_{\widetilde{1}}^{*}\right)$ by the map

$$
L_{\widetilde{1}}^{*} \otimes L_{\widetilde{1}}^{*} \longrightarrow \mathbb{P}\left(L_{\tilde{1}} \oplus L_{\widetilde{1}}^{*}\right), \quad\left(\left[v_{\tilde{1}}, v_{\widehat{1}}\right], u\right) \longrightarrow\left[v_{\tilde{1}}, v_{\widehat{1}}+u\left(v_{\tilde{1}}\right)\right] .
$$

Near $\mathcal{Z}_{\mathcal{T}}$,

$$
\begin{aligned}
\mathcal{D}_{\widetilde{1}, \hat{1}} \phi_{\mathcal{T}_{1}, \mathcal{T}}\left(\left[v_{\widetilde{1}}, v_{\widehat{1}}\right] ; u, v\right)= & -\left(y_{\widehat{1}}-x_{h}\right)^{-3} \otimes\left(\mathcal{D}_{\mathcal{T}, h}^{(2)}+\varepsilon_{2}(u, v)\right) v \otimes v \otimes u \\
& -\left(y_{\widehat{1}}-x_{h}\right)^{-2} \otimes v_{\widetilde{1}} \otimes\left(\mathcal{D}_{\mathcal{T}, h}^{(3)}+\varepsilon_{3}(u, v)\right) v \otimes v \otimes v
\end{aligned}
$$

for all $\left(\left[v_{\tilde{1}}, v_{\widehat{1}}\right] ; v\right) \in \mathcal{F} \mathcal{T}_{\delta}$ such that $\phi_{\mathcal{T}_{1}, \mathcal{T}}(v) \in \mathcal{U}_{\mathcal{T}_{1}}^{(1)}$. By Lemma 3.4 the images of $\mathcal{D}_{\mathcal{T}, h}^{(2)}$ and $\mathcal{D}_{\mathcal{T}, h}^{(3)}$ are distinct over $\mathcal{S}_{\mathcal{T} \mid \mathcal{T}_{1}}(\mu)$. Thus, by Proposition 2.18B the decomposition (3.4), and a rescaling of the linear map,

$$
\sum_{\chi_{\mathcal{T}}(\widetilde{1}, \widehat{1})=0} \mathcal{C}_{\mathcal{Z}_{\mathcal{T}}}\left(\mathcal{D}_{\widetilde{1}, \hat{1}}\right)=3\left|\mathcal{S}_{1}\right|
$$

The claim follows from equations (17.12) and (7.13).

Lemma 7.9 The total contribution to $\mathcal{C}_{\partial \mathbb{P}\left(L_{\tilde{1}} \oplus L_{\widetilde{1}}^{*}\right)}\left(\mathcal{D}_{\widetilde{1}, \widehat{1}}\right)$ from the boundary strata $\mathbb{P}\left(L_{\widetilde{1}} \oplus L_{\widetilde{1}}^{*}\right) \mid \mathcal{U}_{\mathcal{T}_{1}, \mathcal{T}}$, where $\mathcal{T}=\left(M_{1}, I ; j, \underline{d}\right)$ is a bubble type such that $\mathcal{T}<\mathcal{T}_{1}$ and $\chi_{\mathcal{T}}(\widetilde{1}, \widehat{1})>0$, is given by

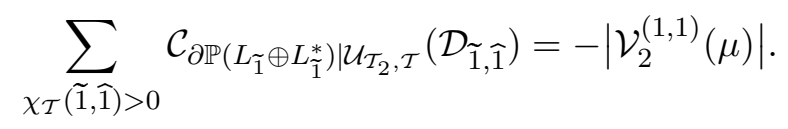


Proof (1) By Lemma 4.1

$$
\overline{\mathcal{U}}_{\mathcal{T}_{1}}^{(1)}(\mu) \cap \mathcal{U}_{\mathcal{T}_{1}, \mathcal{T}} \subset \mathcal{U}_{\mathcal{T} \mid \mathcal{T}_{1}}^{(1)}(\mu)
$$

If $j_{\widehat{1}}=\widetilde{1}$ or $j_{\widehat{1}}>\widetilde{1}$ and $d_{j_{\widehat{1}}}=0$, the section $\mathcal{D}_{\widetilde{1}, \widehat{1}}$ has a nonvanishing extension over $\mathcal{U}_{\mathcal{T} \mid \mathcal{T}_{1}}^{(1)}(\mu)$. Thus, we only need to consider bubble types $\mathcal{T}$ such that

$$
j_{\widehat{1}} \equiv h>\widetilde{1} \quad \text { and } \quad d_{h}>0 .
$$

Furthermore, if $\mathcal{U}_{\mathcal{T} \mid \mathcal{T}_{1}}^{(1)}(\mu) \neq \emptyset, I^{+}=\{\widetilde{1}, h\}$.

(2) If $d_{\widetilde{0}}=0$ and $\mathcal{U}_{\mathcal{T} \mid \mathcal{T}_{1}}^{(1)}(\mu) \neq \emptyset, \mathcal{T}=\mathcal{T}_{1}(l)$ for some $l \in[N]$. By Proposition 3.5 .

$$
\begin{gathered}
\mathcal{D}_{\widetilde{1}, \hat{1}} \phi_{\mathcal{T}_{1}, \mathcal{T}}\left(\left[v_{\widetilde{1}}, v_{\widehat{1}}\right] ; v\right)=-\left(y_{\widehat{1}}-x_{h}\right)^{-2}\left\{v_{\widehat{1}} \otimes \mathcal{D}_{\mathcal{T}, h}^{(1)}+\varepsilon(v)\right\} v^{*} \\
\text { for all } v \in \mathcal{F} \mathcal{T}_{\delta}-Y\left(\mathcal{F} \mathcal{T} ; \widehat{I}^{+}\right)
\end{gathered}
$$

Let $\mathcal{Z}_{\mathcal{T}}=\mathbb{P} L_{\widetilde{1}}$. Since the section $\mathcal{D}_{\mathcal{T}, h}^{(1)}$ does not vanish on $\mathcal{U}_{\mathcal{T} \mid \mathcal{T}_{1}}^{(1)}(\mu)$, by Proposition $2.18 \mathrm{~B}$ and a rescaling of the linear map,

$$
\begin{gathered}
\mathcal{C}_{\mathbb{P}\left(L_{\widetilde{1}} \oplus L_{\widehat{1}}^{*}\right) \mid \mathcal{U}_{\mathcal{T} \mid \mathcal{T}_{1}}^{(1)}(\mu)-\mathcal{Z}_{\mathcal{T}}}\left(\mathcal{D}_{\widetilde{1}, \widehat{1}}\right)=-N\left(\alpha_{1}\right), \quad \text { where } \\
\alpha_{1} \in \Gamma\left(\mathbb{P}^{1} \times \mathcal{S}_{1}(\mu) ; \operatorname{Hom}\left(\gamma^{*}, \gamma^{*} \otimes \operatorname{ev}_{\widehat{0}}^{*} T \mathbb{P}^{2}\right)\right)
\end{gathered}
$$

is a nonvanishing section. Thus, by Proposition 2.18A

$$
\mathcal{C}_{\mathbb{P}\left(L_{\tilde{1}} \oplus L_{\widetilde{1}}^{*}\right) \mid \mathcal{U}_{\mathcal{T} \mid \mathcal{T}_{1}}^{(1)}(\mu)-\mathcal{Z}_{\mathcal{T}}}\left(\mathcal{D}_{\widetilde{1}, \hat{1}}\right)=-\left\langle 2 \lambda-\lambda, \mathbb{P}^{1}\right\rangle\left|\mathcal{U}_{\mathcal{T} \mid \mathcal{T}_{1}}^{(1)}(\mu)\right|=-\left|\mathcal{U}_{\mathcal{T} \mid \mathcal{T}_{1}}^{(1)}(\mu)\right| .
$$

On the other hand, with the same notation as in (2) of the proof of Lemma 7.8 , near $\mathcal{Z}_{\mathcal{T}}$

$$
\begin{aligned}
\mathcal{D}_{\tilde{1}, \widehat{1}} \phi_{\mathcal{T}_{1}, \mathcal{T}}\left(\left[v_{\tilde{1}}, v_{\widehat{1}}\right] ; u, v\right)=-\left(y_{\widehat{1}}-x_{h}\right)^{-2}\left\{\mathcal{D}_{\mathcal{T}, h}^{(1)}\right. & \left.+\varepsilon_{\widehat{1}}(v)\right\} u \otimes v^{*} \\
& +\left\{\mathcal{D}_{\mathcal{T}, h}^{(1)}+\varepsilon_{\widetilde{1}}(v)\right\} v .
\end{aligned}
$$

Thus, by Proposition 2.18B

$$
\mathcal{C}_{\mathcal{Z}_{\mathcal{T}}}\left(\mathcal{D}_{\tilde{1}, \hat{1}}\right)=\left|\mathcal{Z}_{\mathcal{T}}\right|=\left|\mathcal{U}_{\mathcal{T} \mid \mathcal{T}_{1}}^{(1)}(\mu)\right| .
$$

We conclude that

$$
\sum_{d_{\tilde{1}}=0} \mathcal{C}_{\mathbb{P}\left(L_{\widetilde{1}} \oplus L_{\widetilde{1}}^{*}\right) \mid \mathcal{U}_{\mathcal{T}_{1}, \mathcal{T}}}\left(\mathcal{D}_{\widetilde{1}, \hat{1}}\right)=0
$$

(3) Finally, suppose $d_{\tilde{1}}>0$. The same argument as in (2) above shows that

$$
\mathcal{C}_{\mathbb{P}\left(L_{\tilde{1}} \oplus L_{\widetilde{1}}^{*}\right) \mid \mathcal{U}_{\mathcal{T} \mid \mathcal{T}_{1}}^{(1)}(\mu)-\mathcal{Z}_{\mathcal{T}}}\left(\mathcal{D}_{\widetilde{1}, \widehat{1}}\right)=-\left|\mathcal{U}_{\mathcal{T} \mid \mathcal{T}_{1}}^{(1)}(\mu)\right|
$$


but $\mathcal{Z}_{\mathcal{T}}$ is $\mathcal{D}_{\tilde{1}, \hat{1}}$-hollow. Thus, summing up over all bubble types $\mathcal{T}$ of appropriate form, we obtain

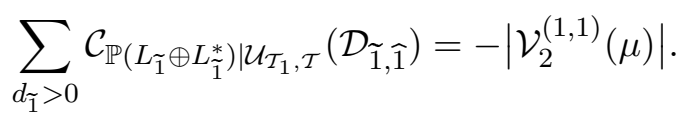

The claim follows equations (7.14) and (7.15).

\subsection{Rational cuspidal curves in $\mathbb{P}^{n}$}

In this subsection, we prove Theorem [1.1 In particular, we construct a tree of chern classes, as mentioned in the third-to-last paragraph of Subsection 1.2 . The sum of these chern classes, with an appropriate sign, is the number that appears on the right-hand side of the equation in Theorem 1.1. The tree is very similar to that constructed in Subsection 3.1 of [20]; the main difference is that here we focus on intersection numbers, instead of zeros of polynomial maps. Theorem 1.1 follows immediately from Corollary 7.12 and Lemma 7.13

We first introduce a little more notation. If $d, N$, and $\mu$ are as in the statement of Theorem 1.1, $k \geq 1$, and $m \geq 0$, let $\mathcal{V}_{k, m}(\mu)$ and $\overline{\mathcal{V}}_{k, m}(\mu)$ denote the quotients of the disjoint unions of the spaces $\mathcal{U}_{\mathcal{T}}(\widetilde{\mu})$ and $\overline{\mathcal{U}}_{\mathcal{T}}(\widetilde{\mu})$, respectively, taken over all bubble types

$$
\begin{gathered}
\mathcal{T}=\left([N]-M_{0}, I_{k} ; j, \underline{d}\right) \quad \text { such that } \quad M_{0} \subset[N],\left|M_{0}\right|=m, \\
I_{k}=\{\widehat{0}\} \cup\{\widetilde{1}, \ldots, \widetilde{k}\}, \quad d_{\tilde{1}}, \ldots, d_{\widetilde{k}}>0, \quad \sum d_{i}=d,
\end{gathered}
$$

by the natural action of the symmetric group $S_{k}$. Here $\widetilde{\mu}$ is the $\left([N]-M_{0}\right) \sqcup\{\widehat{0}\}-$ tuple of constraints defined by

$$
\widetilde{\mu}_{l}=\mu_{l} \quad \text { if } l \in[N]-M_{0} ; \quad \widetilde{\mu}_{\widehat{0}}=\bigcap_{l \in M_{0}} \mu_{l} .
$$

By dimension-counting, the spaces $\mathcal{V}_{k, m}(\mu)$ are smooth manifolds. We define the vector bundle $E_{k, m} \rightarrow \overline{\mathcal{V}}_{k, m}(\mu)$ and homomorphism $\alpha_{k, m}: E_{k, m} \rightarrow \operatorname{ev}_{\widehat{0}}^{*} T \mathbb{P}^{n}$ over $\overline{\mathcal{V}}_{k, m}(\mu)$ by

$$
E_{k, m} \mid \overline{\mathcal{U}}_{\mathcal{T}}(\mu)=\bigoplus_{i \in I^{+}} L_{i}, \quad \alpha_{k, m}\left(\left(v_{i}\right)_{i \in I^{+}}\right)=\sum_{i \in I^{+}} \mathcal{D}_{\mathcal{T}, i}^{(1)} v_{i},
$$

whenever $\mathcal{T}$ is a bubble type as above.

We now construct the tree mentioned above. Each node is a tuple

$$
\sigma=(r ; k, m ; \phi),
$$


where $r \geq 0$ is the distance to the root $\sigma_{0}=(0 ; 1,0 ; \cdot), k \geq 1$, and $m \geq 0$. The tree satisfies the following properties. If $r>0$ and

$$
\sigma^{*} \equiv\left(r-1 ; k^{*}, m^{*} ; \phi^{*}\right)
$$

is the node from which $\sigma$ is directly descendant, we require that $k^{*} \leq k$, $m^{*} \leq m$, and at least one of the inequalities is strict. Furthermore, $\phi$ specifies a splitting of the set $[k]$ into $k^{*}$ disjoint subsets and an assignment of $m-m^{*}$ of the elements of the set

$$
[m] \equiv\{(1,1), \ldots,(1, m)\}
$$

to these subsets. This description inductively constructs an infinite tree. However, we will need to consider only the nodes

$$
\sigma \equiv(r ; k, m ; \phi) \quad \text { such that } 2 k+m \leq n+2 .
$$

We will write $\sigma \vdash \sigma^{*}$ to indicate that $\sigma$ is directly descendant from $\sigma^{*}$.

For each node in the above tree, except for the root, we now define a linear map between vector bundles. If $\sigma=(r ; k, m ; \phi)$ and $s \geq 1$, let

$$
\left\{\sigma_{s}=\left(s ; k_{s}, m_{s} ; \phi_{s}\right): 0 \leq s \leq r\right\}
$$

be the sequence of nodes such that $\sigma_{r}=\sigma$ and $\sigma_{s} \vdash \sigma_{s-1}$ for all $s>0$. Put

$$
\begin{gathered}
\overline{\mathcal{V}}_{\sigma}=\overline{\mathcal{V}}_{k, m}(\mu), \quad E_{\sigma}=E_{k, m} \longrightarrow \overline{\mathcal{V}}_{\sigma}, \quad \alpha_{\sigma}=\alpha_{k, m}, \\
\mathcal{X}_{\sigma}=\mathcal{Y}_{\sigma} \times \overline{\mathcal{V}}_{\sigma}, \quad \mathcal{X}_{\sigma, s}=\mathcal{Y}_{\sigma, s} \times \overline{\mathcal{V}}_{\sigma},
\end{gathered}
$$

where

$$
\begin{gathered}
\mathcal{Y}_{\sigma}=\mathcal{Y}_{\sigma, r}, \quad \mathcal{Y}_{\sigma, 0}=\{p t\}, \quad \mathcal{Y}_{\sigma, s}=\mathbb{P} F_{\sigma s} \times \mathcal{Y}_{\sigma, s-1} \quad \text { if } s>0, \\
\overline{\mathfrak{M}}_{\sigma}=\prod_{i \in \operatorname{Im} \phi} \overline{\mathfrak{M}}_{i+\phi^{-1}(i)}, \quad F_{\sigma}=\bigoplus_{i \in \operatorname{Im} \phi} \gamma_{\sigma ; i} \longrightarrow \overline{\mathfrak{M}}_{\sigma} .
\end{gathered}
$$

For the purposes of the last line above, we view $\phi$ as a map from $[k]-\left[k^{*}\right]$ and a subset of $[m]$ to $\left[k^{*}\right]$ in the notation of the previous paragraph. Then,

$$
\gamma_{\sigma ; i} \longrightarrow \overline{\mathfrak{M}}_{i+\phi^{-1}(i)}
$$

is the "tautological" line bundle, ie the universal tangent bundle at the marked point $i$. Let

$$
\mathcal{O}_{\sigma}=\mathcal{O}_{\sigma, r}, \quad \mathcal{O}_{\sigma, 1}=\operatorname{ev}_{\widehat{0}}^{*} T \mathbb{P}^{n}, \quad \mathcal{O}_{\sigma, s}=\mathcal{O}_{\sigma, s-1} / \operatorname{Im} \bar{\nu}_{\sigma, s-1} \text { if } s>1,
$$

where $\bar{\nu}_{\sigma, s} \in \Gamma\left(\mathcal{X}_{\sigma, s} ; \operatorname{Hom}\left(\gamma_{F_{\sigma_{s}}}, \mathcal{O}_{\sigma, s}\right)\right)$ is a generic section. Since $k_{s-1} \leq k_{s}$, $m_{s-1} \leq m_{s}$, and one of the inequalities is strict,

$$
\begin{aligned}
\frac{1}{2} \operatorname{dim} \mathcal{X}_{\sigma, s} \leq \frac{1}{2} \operatorname{dim} \mathcal{X}_{\sigma} & =(n+2-2 k-m)+\sum_{s=1}^{s=r}\left(\left|\operatorname{Im} \phi_{s}\right|-1\right) \\
& =n+1-k-r<\operatorname{rk} \mathcal{O}_{\sigma, 1}-(r-1) .
\end{aligned}
$$


Thus, we see inductively that each bundle $\mathcal{O}_{\sigma, s}$ is well-defined and a generic section $\bar{\nu}_{\sigma, s}$ of $\operatorname{Hom}\left(\gamma_{F_{\sigma, s}}, \mathcal{O}_{\sigma, s}\right)$ does not vanish. Let

$$
\pi_{\sigma}: \mathrm{ev}_{\widehat{0}}^{*} T \mathbb{P}^{n} \longrightarrow \mathcal{O}_{\sigma}
$$

be the projection map. We define

$$
\begin{gathered}
\widetilde{\alpha}_{\sigma} \in \Gamma\left(\mathcal{X}_{\sigma} ; \operatorname{Hom}\left(\gamma_{F_{\sigma}}^{*} \otimes E_{\sigma} ; \gamma_{F_{\sigma}}^{*} \otimes \mathcal{O}_{\sigma}\right)\right) \quad \text { by } \\
\left\{\widetilde{\alpha}_{\sigma}(\tau \otimes v)\right\}(w)=\tau(w) \cdot \pi_{\sigma} \alpha_{\sigma}(v) \in \mathcal{O}_{\sigma} .
\end{gathered}
$$

Lemma 7.10 With notation as above,

$$
\left|\mathcal{S}_{1}(\mu)\right|=\left\langle c\left(L_{\widetilde{1}}^{*} \otimes \operatorname{ev}_{\widehat{0}}^{*} T \mathbb{P}^{n}\right), \overline{\mathcal{V}}_{1}(\mu)\right\rangle-\sum_{\sigma \vdash \sigma_{0}} N\left(\widetilde{\alpha}_{\sigma}\right) .
$$

Furthermore, for every node $\sigma^{*} \neq \sigma_{0}$,

$$
N\left(\widetilde{\alpha}_{\sigma^{*}}\right)=\left\langle c\left(\gamma_{F_{\sigma^{*}}}^{*} \otimes \mathcal{O}_{\sigma^{*}}\right) c\left(\gamma_{F_{\sigma^{*}}}^{*} \otimes E_{\sigma^{*}}\right)^{-1}, \mathcal{X}_{\sigma^{*}}\right\rangle-\sum_{\sigma \vdash \sigma^{*}} N\left(\widetilde{\alpha}_{\sigma}\right) .
$$

Proof This lemma is obtained by the usual argument from the estimate (3b) of Proposition 3.5 via Propositions 2.18A and 2.18B. If $\sigma^{*} \neq \sigma_{0}$, the proof is the same as the proof of Lemma 3.3 in [20]. For the first identity, apply the proof of Lemma 3.3 with $\alpha_{\sigma_{0}}^{\perp}=\mathcal{D}_{\mathcal{T}, \tilde{1}}^{(1)}$.

Lemma 7.11 For every node $\sigma \neq \sigma_{0}$,

$$
\left\langle c\left(\gamma_{F_{\sigma}}^{*} \otimes \mathcal{O}_{\sigma}\right) c\left(\gamma_{F_{\sigma}}^{*} \otimes E_{\sigma}\right)^{-1}, \mathcal{X}_{\sigma}\right\rangle=\left\langle c\left(\operatorname{ev}_{\widehat{0}}^{*} T \mathbb{P}^{n}\right) c\left(E_{k, m}\right)^{-1}, \overline{\mathcal{V}}_{k, m}(\widetilde{\mu})\right\rangle .
$$

Furthermore,

$$
\left\langle c_{1}\left(L_{\widetilde{1}}^{*} \otimes \operatorname{ev}_{\widehat{0}}^{*} T \mathbb{P}^{n}\right), \overline{\mathcal{V}}_{1}(\mu)\right\rangle=\left\langle c\left(\operatorname{ev}_{\widehat{0}}^{*} T \mathbb{P}^{n}\right) c\left(E_{1,0}\right)^{-1}, \overline{\mathcal{V}}_{1,0}(\widetilde{\mu})\right\rangle .
$$

Proof For the first identity, see the proof of [20, Corollary 3.5]. The second equality is clear from the fact that $\operatorname{dim} \overline{\mathcal{V}}_{1}(\mu)=\operatorname{rkev}_{\widehat{0}}^{*} T \mathbb{P}^{n}$

\section{Corollary 7.12}

$$
\begin{aligned}
\left|\mathcal{S}_{1}(\mu)\right|=\sum_{(1,0) \leq(k, m)}\left\{(-1)^{k+m-1} k^{m}(m-1) !\right. \\
\left.\quad \times \sum_{l=0}^{n+2-(2 k+m)}\left(\begin{array}{c}
n+1 \\
l
\end{array}\right)\left\langle a^{l} \widetilde{\eta}_{\widehat{0}, n+2-(2 k+m)-l}, \overline{\mathcal{V}}_{k, m}(\widetilde{\mu})\right\rangle\right\} .
\end{aligned}
$$


Proof This corollary follows from Lemma 7.10 and Lemma 7.11 via straightforward combinatorics; see [20, Corollary 3.6 and Lemma 3.7].

Lemma 7.13 For all $k \geq 1$ and $l \geq 0$,

$$
\begin{aligned}
\sum_{m \geq 0}(-1)^{m} k^{m}(m-1) !\left\langle a^{l} \widetilde{\eta}_{\widehat{0}, n+2-(2 k+m)-l}, \overline{\mathcal{V}}_{k, m}(\widetilde{\mu})\right\rangle \\
=(k-1) !\left\langle a^{l} \eta_{\widehat{0}, n+2-2 k-l}, \overline{\mathcal{V}}_{k}(\mu)\right\rangle
\end{aligned}
$$

Proof See the proof of Corollary 3.10 in [20, which uses (3.2) along with [1], Subsection 3.2].

\section{Low-degree numbers}

We now give some low-degree enumerative numbers for rational curves in projective spaces. In all five tables, the top row lists the degree $d$ of the map. In Tables 11 and 2, the constraints are assumed to be $3 d-2$ points in general position in $\mathbb{P}^{n}$. In Tables 3 and 4 , the constraints are $p$ points and $q$ lines in $\mathbb{P}^{3}$, as specified by the second row. Similarly, in Table 5 , the constraints are $p$ points, $q$ lines, and $r$ two-planes in $\mathbb{P}^{4}$.

The formulas of Theorems 1.2 and 1.3 give zeros in degrees one, two, and three. From classical algebraic geometry, one would expect these low-degree numbers, as well as the first three degree-four numbers listed in Tables 3 and 4, to vanish. In addition, as expected, the fourth number in Table 3 (Table 4) is the same as the degree-four number of Table 1 (Table 2). Similarly, all degree-one and -two numbers $\left|\mathcal{S}_{1}(\mu)\right|$ and several degree-three and -four numbers, as listed in Table 5. are zero, as the case should be. Finally, observe that the third number of Table 5 is the same as the long-known number of plane cubic cuspidal curves that pass through seven general points.

\begin{tabular}{||c|c|c|c|c|c|c|c|c||}
\hline \hline$d$ & 1 & 2 & 3 & 4 & 5 & 6 & 7 & 8 \\
\hline$\left|\mathcal{V}_{1}^{(2)}(\mu)\right|$ & 0 & 0 & 0 & 60 & 56,400 & $49,177,440$ & $56,784,765,120$ & $91,466,185,097,280$ \\
\hline \hline
\end{tabular}

Table 1: One-component rational triple-pointed curves in $\mathbb{P}^{2}$ 


\begin{tabular}{||c|c|c|c|c|c|c|c|c||}
\hline \hline$d$ & 1 & 2 & 3 & 4 & 5 & 6 & 7 & 8 \\
\hline$\left|\mathcal{S}_{1}^{(1)}(\mu)\right|$ & 0 & 0 & 0 & 1,296 & 499,680 & $271,751,040$ & $227,509,931,520$ & $287,190,836,432,640$ \\
\hline \hline
\end{tabular}

Table 2: One-component rational tacnodal curves in $\mathbb{P}^{2}$

\begin{tabular}{||c|c|c|c|c|c|c|c|c|c|c||}
\hline \hline$d$ & 4 & 4 & 4 & 4 & 4 & 4 & 5 & 5 & 5 & 6 \\
\hline$(p, q)$ & $(6,1)$ & $(5,3)$ & $(4,5)$ & $(3,7)$ & $(2,9)$ & $(1,11)$ & $(8,1)$ & $(7,3)$ & $(6,5)$ & $(10,1)$ \\
\hline$\frac{1}{6}\left|\mathcal{V}_{1}^{(2)}(\mu)\right|$ & 0 & 0 & 0 & 60 & 1,280 & 19,640 & 8 & 264 & 4,360 & 4,680 \\
\hline \hline
\end{tabular}

Table 3: One-component rational triple-pointed curves in $\mathbb{P}^{3}$

\begin{tabular}{||c|c|c|c|c|c|c|c|c|c|c||}
\hline \hline$d$ & 4 & 4 & 4 & 4 & 4 & 4 & 5 & 5 & 5 & 6 \\
\hline$(p, q)$ & $(6,1)$ & $(5,3)$ & $(4,5)$ & $(3,7)$ & $(2,9)$ & $(1,11)$ & $(8,1)$ & $(7,3)$ & $(6,5)$ & $(10,1)$ \\
\hline$\frac{1}{2}\left|\mathcal{S}_{1}^{(1)}(\mu)\right|$ & 0 & 0 & 0 & 1,296 & 27,648 & 426,672 & 960 & 9,792 & 111,840 & 112,320 \\
\hline \hline
\end{tabular}

Table 4: One-component rational tacnodal curves in $\mathbb{P}^{3}$

\begin{tabular}{||c|c|c|c|c|c|c|c|c|c|}
\hline \hline$d$ & 3 & 3 & 3 & 3 & 4 & 4 & 4 & 4 & 5 \\
\hline$(p, q, r)$ & $(4,0,1)$ & $(3,1,2)$ & $(3,0,4)$ & $(2,1,5)$ & $(6,0,0)$ & $(5,1,1)$ & $(5,0,3)$ & $(4,1,4)$ & $(7,1,0)$ \\
\hline$\left|\mathcal{S}_{1}(\mu)\right|$ & 0 & 0 & 24 & 240 & 0 & 0 & 0 & 1,680 & 120 \\
\hline \hline
\end{tabular}

Table 5: One-component rational cuspidal curves in $\mathbb{P}^{4}$

\section{References}

[1] Raoul Bott, Loring W Tu, Differential forms in algebraic topology, volume 82 of Graduate Texts in Mathematics, Springer-Verlag, New York (1982) MathReview

[2] Steven Diaz, Joe Harris, Geometry of the Severi variety, Trans. Amer. Math. Soc. 309 (1988) 1-34 MathReview

[3] W Fulton, R Pandharipande, Notes on stable maps and quantum cohomology, from: "Algebraic geometry-Santa Cruz 1995", Proc. Sympos. Pure Math. 62, Amer. Math. Soc., Providence, RI (1997) 45-96 MathReview

[4] Phillip Griffiths, Joseph Harris, Principles of algebraic geometry, Wiley Classics Library, John Wiley \& Sons Inc., New York (1994) MathReview

[5] M Gromov, Pseudoholomorphic curves in symplectic manifolds, Invent. Math. 82 (1985) 307-347 MathReview

[6] Sheldon Katz, Zhenbo Qin, Yongbin Ruan, Enumeration of nodal genus-2 plane curves with fixed complex structure, J. Algebraic Geom. 7 (1998) 569-587 MathReview 
[7] Maxim Kontsevich, Yu Manin, Gromov-Witten classes, quantum cohomology, and enumerative geometry, Comm. Math. Phys. 164 (1994) 525-562 MathReview

[8] Maxim Kontsevich, Enumeration of rational curves via torus actions, from: "The moduli space of curves (Texel Island, 1994)", Progr. Math. 129, Birkhäuser Boston, Boston, MA (1995) 335-368 MathReview

[9] Jun Li, Gang Tian, Virtual moduli cycles and Gromov-Witten invariants of general symplectic manifolds, from: "Topics in symplectic 4-manifolds (Irvine, CA, 1996)", First Int. Press Lect. Ser., I, Internat. Press, Cambridge, MA (1998) 47-83 MathReview

[10] Dusa McDuff, Dietmar Salamon, J-holomorphic curves and quantum cohomology, volume 6 of University Lecture Series, American Mathematical Society, Providence, RI (1994) MathReview

[11] Rahul Pandharipande, Intersections of Q-divisors on Kontsevich's moduli space $\bar{M}_{0, n}\left(\mathbf{P}^{r}, d\right)$ and enumerative geometry, Trans. Amer. Math. Soc. 351 (1999) 1481-1505 MathReview

[12] Ziv Ran, On the quantum cohomology of the plane, old and new, and a K3 analogue, Collect. Math. 49 (1998) 519-526 MathReview

[13] Ziv Ran, Enumerative geometry of divisorial families of rational curves, Ann. Sc. Norm. Super. Pisa Cl. Sci. (5) 3 (2004) 67-85 MathReview

[14] Yongbin Ruan, Gang Tian, A mathematical theory of quantum cohomology, J. Differential Geom. 42 (1995) 259-367 MathReview

[15] R Vakil, Enumerative Geometry of Plane Curve of Low Genus, preprint, arXiv:math.AG/9803007

[16] H Zeuthen, Almindelige Egenskaber ved Systemer af Plane Kurver, Kongelige Danske Videnskabernes Selskabs Skrifter, 10 (1873) 285-393 (Danish)

[17] Aleksey Zinger, Enumeration of genus-two curves with a fixed complex structure in $\mathbb{P}^{2}$ and $\mathbb{P}^{3}$, J. Differential Geom. 65 (2003) 341-467 MathReview

[18] Aleksey Zinger, Enumerative vs. Symplectic Invariants and Obstruction Bundles, to appear in J. Symplectic Geom.

[19] Aleksey Zinger, Enumeration of genus-three plane curves with a fixed complex structure, J. Algebraic Geom. 14 (2005) 35-81 MathReview

[20] Aleksey Zinger, Enumeration of one-nodal rational curves in projective spaces, Topology 43 (2004) 793-829 MathReview 